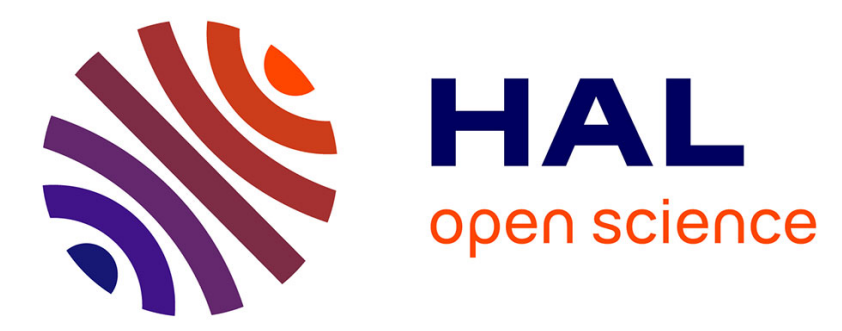

\title{
Notes on Metric Independent Analysis of Classical Fields Reuven Segev
}

\section{To cite this version:}

Reuven Segev. Notes on Metric Independent Analysis of Classical Fields. Mathematical Methods in the Applied Sciences, 2013, 36 (5), pp.497-566. hal-01068262

\section{HAL Id: hal-01068262 https://hal.science/hal-01068262}

Submitted on 25 Sep 2014

HAL is a multi-disciplinary open access archive for the deposit and dissemination of scientific research documents, whether they are published or not. The documents may come from teaching and research institutions in France or abroad, or from public or private research centers.
L'archive ouverte pluridisciplinaire HAL, est destinée au dépôt et à la diffusion de documents scientifiques de niveau recherche, publiés ou non, émanant des établissements d'enseignement et de recherche français ou étrangers, des laboratoires publics ou privés. 


\title{
NOTES ON METRIC INDEPENDENT ANALYSIS OF CLASSICAL FIELDS
}

\author{
REUVEN SEGEV
}

\begin{abstract}
A metric independent geometric framework for some fundamental objects of continuum mechanics is presented. In the geometric setting of general differentiable manifolds, balance principles for extensive properties are formulated and Cauchy's theorem for fluxes is proved. Fluxes in an $n$-dimensional space are represented as differential $(n-\mathrm{I})$-forms. In an analogous formulation of stress theory, a distinction is made between the traction stress, enabling the evaluation of the traction on the boundaries of the various regions, and the variational stress which acts on the derivative of a virtual velocity field to produce the virtual power density. The relation between the two stress fields is examined as well as the resulting differential balance law. As an application, metric invariant aspects of electromagnetic theory are presented within the framework of the foregoing flux and stress theory.
\end{abstract}

\section{INTRODUCTION}

These notes present a formulation of various notions of continuum physics on differentiable manifolds devoid of a metric structure. In particular, we concentrate our attention on flux fields and stress fields. An effort was made to make the notes self-contained and so they contain an introduction to some of the elements of the theory of differentiable manifolds and integration theory of differential forms. As an example, the last section considers in some detail metric-independent electromagnetic theory, or premetric electrodynamics as the subject is referred to in recent work (e.g., [HIO O6]). The notes are intended for a reader who is already familiar with the fundamentals of continuum mechanics as formulated in a 3-dimensional Euclidean space. Thus, an attempt was made to make the text informal and occasionally, we mention the relevance of a mathematical construction to mechanics.

Metric independent continuum mechanics has the advantage of being applicable to both classical and relativistic theories. In The Classical Field Theories, [TT60, p. 66o], Truesdell and Toupin wrote:

"A principal objective is to isolate those aspects of the theory which are independent of the assigned geometry of space-time from those whose formulation and interpretation depend on or imply a particular space-time geometry. For example, we

Key words and phrases. Continuum mechanics; flux; stress; balance principles; differentiable manifolds; differential forms; premetric electromagnetism. 
regard the conservation of charge as a physical or intuitive concept logically independent of the concepts of rigid rods, uniform clocks, and inertial frames, and we have chosen to express this law in a mathematical form likewise independent of the representation of these extraneous entities."

A second fundamental advantage of a metric free framework for continuum mechanics involves the notion of a body. While in older work in solid mechanics the existence of a natural stress free configuration of a body has been taken for granted, it was recognized that for a general theory no preferred reference configuration of a body of material is available. As early as 1957, Walter Noll [Nol59] defined a body in continuum mechanics as a differentiable manifold whose configurations in space serve as charts. In a metric independent formulation of continuum mechanics, various fields such as flux fields and stress fields may be represented by objects independent of configuration. For example, the transformations of vector fields associated with electromagnetic theory from the Eulerian description to the Lagrangian description in [DOo5, DOo9] should be viewed merely as transformation rules for the components of tensors under coordinate transformations. A flux field, such as the heat flux field, should be regarded as a 2-form in the 3dimensional body rather than as a vector field. As a 2-form, a heat flux field may be considered independently of the configuration of the body in space.

This review begins with algebraic Cauchy's theorem, i.e., Cauchy's flux theorem for uniform fluxes. The theorem is considered in Section 3 following a short introduction in Section 2 to simplices in affine spaces. The theorem motivates the study of alternating tensors which follows in Section 4 . Sections 5 and 6 continue the introduction of the mathematical framework and they consider the basic elements of differentiable manifolds and integration theory of differential forms defined on them. Section 7 considers scalar balance laws. Cauchy's theorem for fluxes of scalar valued extensive properties is proved and the differential balance law is written using the exterior derivative. Some properties of fluxes are studied in Section 8 and Section 9 presents the material structure induced by the flux of an extensive property. In the preceding sections it was convenient to view the manifold under consideration as the space manifold or as a body manifold. In Section 9, the balance equations are formulated in spacetime manifold. Section Io introduces force densities and stresses. In a Euclidean space, the stress tensor plays two roles. On the one hand it gives the traction on the boundary of subbodies using Cauchy's formula and on the other hand, it acts on the gradient of a virtual velocity field to produce virtual power density in the bulk. If a Riemannian metric is not available, the object that specifies the traction fields, to which we refer as the traction stress is different from the object that produces virtual power - the variational stress. These two objects and the relations between them are studied, and the divergence of the variational stress is defined. Finally, in Section II we see how the notions introduced earlier apply to a metric independent formulation of elements of electromagnetic theory.

These notes use material that was presented in some earlier papers, notably, [Segoo, SR99, Sego2, SRo2]. For expositions of continuum mechanics from the differential geometric point of view that develop other aspects of the theory the 
reader is referred to [ $\left.\mathrm{MH}_{94}\right]$ and to the more recent [Epsio]. Both [MH94] and [Epsio] contain introductions to differential geometry. As general introductory texts to differential geometry, the reader may further consult [AMR88, AM77, BC7O, FraO4, LeeO2, War83].

\section{Simplexes in Vector Spaces and Their Boundaries}

2.I. Affine Spaces. We recall that an affine space A having a translation space $\mathbf{V}$ may be thought of as a vector space for which a particular zero vector has not been chosen. (See for example [AM77, pp. 2-6] [Whi57, pp. 349-35I].) For each ordered pair $\left(p, p^{\prime}\right)$ of points in $\mathbf{A}$ there is a vector $v=p^{\prime}-p \in \mathbf{V}$ which we regard as pointing from $p$ to $p^{\prime}$ and we can write $p^{\prime}=p+v$. A choice of a particular point $p_{0}$ induces a bijection of $\mathbf{A}$ and $\mathbf{V}$ by $p \mapsto p-p_{0}$. This bijection is meaningful as for any 3 points $p, p^{\prime}, p^{\prime \prime} \in \mathbf{A}$,

$$
p^{\prime \prime}-p=\left(p^{\prime \prime}-p^{\prime}\right)+\left(p^{\prime}-p\right),
$$

where it is noted the plus sign above is the addition in the vector space $\mathbf{V}$ while the minus sign is the basic operation taking a pair of points in the affine space $\mathbf{A}$ and giving a vector in $\mathbf{V}$. In particular, it follows from Equation (2.I) that

$$
p-p=0, \quad p-p^{\prime}=-\left(p^{\prime}-p\right), \quad \text { and } \quad(p-q)-\left(p^{\prime}-q\right)=p-p^{\prime} .
$$

The affine space $\mathbf{A}$ is said to be $n$-dimensional if $\mathbf{V}$ is $n$-dimensional.

It is emphasized that the difference between an affine space and a Euclidean space is that an affine space does not have a metric structure. Notions such as distance, length, angle, extension, and shear are meaningless.

2.2. Simplices. Let $\mathbf{A}$ be an $n$-dimensional affine space and $p_{0}, \ldots, p_{r} \in \mathbf{A}$ be $r+1$ points such that the vectors $v_{i}=p_{i}-p_{0} \in \mathbf{V}, i=1, \ldots, r$, are linearly independent. Thus, the vectors $v_{i}$ may serve as a basis for the subspace of $\mathbf{V}$ containing them. If one adds the vectors of this subspace to $p_{0}$ one obtains an $r$-dimensional affine subspace of $\mathbf{A}$ containing $p_{0}$.

The simplex generated by the ordered sequence of points $p_{0}, \ldots, p_{r}$ contains the set

$$
s=\left\{p_{0}+\sum_{i=1}^{r} x^{i}\left(p_{i}-p_{0}\right) \mid x^{i} \geqslant 0, \sum_{i=1}^{r} x^{i} \leqslant 1\right\} .
$$

Let $O$ be some origin and $w_{i}=p_{i}-O, i=0, \ldots, r$. Then,

$$
\begin{aligned}
p_{0}+\sum_{i=1}^{r} x^{i}\left(p_{i}-p_{0}\right) & =O+w_{0}+\sum_{i=1}^{r} x^{i}\left(p_{i}-p_{0}\right), \\
& =O+w_{0}+\sum_{i=1}^{r} x^{i}\left(w_{i}-w_{0}\right) \\
& =O+\left(1-\sum_{i=1}^{r} x^{i}\right) w_{0}+\sum_{i=1}^{r} x^{i} w_{i}
\end{aligned}
$$


where the second line follows from Equation (2.2). Setting $x^{0}=1-\sum_{i=1}^{r} x^{i}$, we obtain

$$
p_{0}+\sum_{i=1}^{r} x^{i}\left(p_{i}-p_{0}\right)=O+\sum_{j=0}^{r} x^{j} w_{j}
$$

Since the left-hand side of the last equation is independent of the choice of $O$, the same holds for the right-hand side and we use the notation

$$
\sum_{j=0}^{r} x^{j} p_{j}
$$

for both. Thus, one may define a simplex alternatively as

$$
s=\left\{\sum_{i=0}^{r} x^{i} p_{i} \mid x^{i} \geqslant 0, \sum_{i=0}^{r} x^{i}=1\right\} .
$$

Clearly, the simplex $s$ is defined equivalently by either (I) the collection of points $p_{0}, \ldots, p_{r}$, (2) the point $p_{0}$ and the collection of vectors $v_{i}=p_{i}-p_{0}, i=1, \ldots, r$, (3) the point $p_{0}$ together with the collection of vectors $u_{i}=p_{i}-p_{i-1}, i=1, \ldots, r$. Using the convention $v_{0}=0$, one has $u_{i}=v_{i}-v_{i-1}, v_{i}=\sum_{j=1}^{i} u_{j}$.

We will use the notation

$$
s=\left[p_{0}, \ldots, p_{r}\right]
$$

and in case we are considering a fixed point $p_{0}$ or ignoring it, we will also write (abusing the notation)

$$
s=\left[v_{1}, \ldots, v_{r}\right]=\left[u_{1}, \ldots, u_{r}\right] .
$$

2.3. Cubes, prisms and simplices. The $r$-dimensional cube $e^{\mathrm{I}}$ in $\mathbf{A}$ generated by the points $p_{0}, \ldots, p_{r}$, such that the vectors $u_{i}=p_{i}-p_{0} \in \mathbf{V}, i=1, \ldots, r$ are linearly independent, contains all the points of the form

$$
p_{0}+\sum_{i=1}^{r} x^{i} u_{i}, \quad 0 \leqslant x^{i} \leqslant 1
$$

In analogy, one may consider the open cube for which $0<x^{i}<1$. The points in the cube may be subdivided into prisms. Given an ordered pair $(i, j)$ of indices $0 \leqslant i, j \leqslant r$, the prism $P_{i j}$ is the collection of points of the cube such that $x^{i} \geqslant$ $x^{j}$. The points of the prism may be subdivided further by ordering the rest of the components. Thus, for a sequence of indices $i_{1}, \ldots, i_{r}$ such that $i_{j} \neq i_{k}$ for $j \neq k$, one may consider the set

$$
s_{i_{1} \ldots i_{r}}=\left\{p_{0}+\sum_{j=1}^{r} x^{i_{j}} u_{i_{j}} \mid 1 \geqslant x^{i_{j}} \geqslant 0, x^{i_{1}} \geqslant x^{i_{2}} \geqslant \ldots \geqslant x^{i_{r}}\right\} .
$$

\footnotetext{
IMaybe a "parallelepiped" would describe it better as lengths and angles are meaningless in this setting
} 
We will use the notation $\Pi_{r}$ for the collection of permutations of $r$ ordered symbols (taken as the set $\{1, \ldots, r\}$ ). Thus, every permutation $\pi \in \Pi_{r}$ is a bijection of the form

$$
\pi:\{1, \ldots, r\} \longrightarrow\{1, \ldots, r\}
$$

and we will often write $\pi_{i}$ for $\pi(i), i=1, \ldots, r$. While this is not the traditional notation, we will specify a particular permutation $\pi$ in the form

$$
\pi:(1, \ldots, r) \longmapsto\left(\pi_{1}, \ldots, \pi_{r}\right)=(\pi(1), \ldots, \pi(r)) .
$$

A sequence of $r$ distinct vectors chosen from $u_{1}, \ldots, u_{r}$ is associated with a permutation $\pi$ by $u_{\pi(1)}, \ldots, u_{\pi(i)}, \ldots, u_{\pi(r)}$ so that $\pi(i)$ indicates the vector that occupies the $i$-th position in the sequence. Conversely, $\pi^{-1}(j)$ is the position of $u_{j}$ in the sequence.

Thus, we can write (2.II) alternatively as

$$
s_{\pi}=\left\{p_{0}+\sum_{i=1}^{r} x^{\pi_{i}} u_{\pi_{i}} \mid 1 \geqslant x^{\pi_{i}} \geqslant 0, x^{\pi_{1}} \geqslant x^{\pi_{2}} \geqslant \ldots \geqslant x^{\pi_{r}}\right\}
$$

where $\pi_{j}=\pi(j)=i_{j}$.

Consider the set $s_{\pi}$. If one starts at $p_{0}$, and since $x^{\pi_{1}}$ is not bounded by the other components, the path from $p_{0}$ to $p_{0}+u_{\pi_{1}}=p_{\pi_{1}}$ is contained in the boundary of $s_{\pi}$. We will also use the notation $\left(s_{\pi}\right)_{0}=p_{0}$ and $\left(s_{\pi}\right)_{1}=p_{\pi_{1}}$ as these are the "origin" and first point of $s_{\pi}$. Now that $x^{\pi_{1}}=1$, one can vary $x^{\pi_{2}}$ from 0 to 1 to obtain the path from $p_{\pi_{1}}$ to $\left(s_{\pi}\right)_{2}=p_{0}+u_{\pi_{1}}+u_{\pi_{2}}=\left(s_{\pi}\right)_{1}+u_{\pi_{2}}$. Continuing this procedure $r$ times, one obtains $r$ points $\left(s_{\pi}\right)_{i}, i=1, \ldots, r$ such that

$$
\left(s_{\pi}\right)_{i}=p_{0}+\sum_{j=1}^{i} u_{\pi_{j}}, \quad u_{\pi_{i}}=\left(s_{\pi}\right)_{i}-\left(s_{\pi}\right)_{i-1} .
$$

Clearly, each of these points is located at one of the vertices of the cube.

We will use the notation $v_{\pi_{i}}=\left(s_{\pi}\right)_{i}-\left(s_{\pi}\right)_{0}$ so that

$$
\left(s_{\pi}\right)_{i}=p_{0}+v_{\pi_{i}}, \quad u_{\pi_{i}}=v_{\pi_{i}}-v_{\pi_{i-1}} .
$$

The set $s_{\pi}$ is the simplex determined by the points $p_{0},\left(s_{\pi}\right)_{1},\left(s_{\pi}\right)_{2}, \ldots,\left(s_{\pi}\right)_{r}$. To realize that, and setting $v_{0}=0$ formally, we observe that for $1 \geqslant x^{\pi_{i}} \geqslant 0, x^{\pi_{1}} \geqslant$ 
$x^{\pi_{2}} \geqslant \ldots \geqslant x^{\pi_{r}}$, one has

$$
\begin{aligned}
p \in s_{\pi} & \Leftrightarrow x=p_{0}+\sum_{i=1}^{r} x^{\pi_{i}} u_{\pi_{i}}, \\
& \Leftrightarrow x=p_{0}+\sum_{i=1}^{r} x^{\pi_{i}}\left(v_{\pi_{i}}-v_{\pi_{i-1}}\right), \\
& \Leftrightarrow x=p_{0}+\sum_{i=1}^{r} x^{\pi_{i}} v_{\pi_{i}}-\sum_{i=1}^{r} x^{\pi_{i}} v_{\pi_{i-1}}, \\
& \Leftrightarrow x=p_{0}+\sum_{i=1}^{r} x^{\pi_{i}} v_{\pi_{i}}-\sum_{i=0}^{r-1} x^{\pi_{i+1}} v_{\pi_{i}}, \\
& \Leftrightarrow x=p_{0}+\sum_{i=1}^{r-1}\left(x^{\pi_{i}}-x^{\pi_{i+1}}\right) v_{\pi_{i}}+x^{\pi_{r}} v_{\pi_{r}}, \\
& \Leftrightarrow x=p_{0}+\sum_{i=1}^{r} y^{\pi_{i}} v_{\pi_{i}}, \quad y^{\pi_{i}} \geqslant 0, \sum_{i=1}^{r} y^{\pi_{i}} \leqslant 1
\end{aligned}
$$

where the $y^{\pi_{i}}$ are related to the $x^{\pi_{j}}$ by $y^{\pi_{i}}=x^{\pi_{i}}-x^{\pi_{i+1}}, i=1, \ldots, r-1, y^{\pi_{r}}=x^{\pi_{r}}$, so that $y^{\pi_{i}} \geqslant 0, \sum_{i=1}^{r} y^{\pi_{i}}=x^{\pi_{1}} \leqslant 1$. Conversely, given $y^{\pi_{i}} \geqslant 0$ with $\sum_{i=1}^{r} y^{\pi_{i}} \leqslant 1$, then, $x^{\pi_{i}}=\sum_{j=i}^{r} y^{\pi_{j}} \leqslant 1$ with $x^{\pi_{i}} \geqslant x^{\pi_{i+1}} \geqslant 0$.

The simplices $s_{\pi}$, for the various permutations $\pi \in \Pi_{r}$, cover the cube. They intersect one another on their boundaries where equality signs hold in (2.I4) so that their interiors are disjoint. Since there are $r$ ! permutations of $r$ symbols, there are $r$ ! simplexes of this form in the cube.

Given the prism $P_{i j}$, there are $r ! / 2$ such simplexes that will fit in it-those corresponding to permutations $\pi$ such that $\pi^{-1}(i)<\pi^{-1}(j)$.

2.4. Orientation. An orientation of an affine hyperplane in an affine space gives meanings to notions such as "inside" and "outside" as pertain to to objects such as cubes, simplices, and prisms in that hyperplane. An orientation in an $r$-dimensional hyperplane is determined by a collection of $r$ linearly independent vectors $v_{1}, \ldots, v_{r}$ in the translation space of the hyperplane. As these vectors may be used as a basis, any other collection of $r$ linearly independent vectors, e.g., $u_{1}, \ldots, u_{r}$ may be represented by a matrix $A$ such that $u_{i}=\sum_{j=1}^{r} A_{i}^{j} v_{j}$. If $\operatorname{det}\left(A_{i}^{j}\right)>0$ one says that the collection of vectors $u_{1}, \ldots, u_{r}$ has the same orientation as $v_{1}, \ldots, v_{r}$, alternatively, one says that the basis $u_{1}, \ldots, u_{r}$ has positive orientation relative to the basis $v_{1}, \ldots, v_{r}$. Thus, any other basis of the translation space of the hyperplane may be either of a positive orientation or a negative orientation relative to $v_{1}, \ldots, v_{r}$. Using the elementary properties of determinants, it follows that switching the position of two vectors in a list, reverses the orientation of the collection.

Given a permutation $\pi$, we use the notation $|\pi|$ for the number of swaps or transpositions required to transform $1, \ldots, r$ into $\pi_{1}, \ldots, \pi_{r}$. Clearly, $\left|\pi^{-1}\right|=|\pi|$ and we write

$$
(-1)^{|\pi|}=\operatorname{sign} \pi=\varepsilon_{1 \ldots \ldots r}^{\pi_{1} \ldots \pi_{r}},
$$


where $\varepsilon_{1 \ldots . . r}^{\pi_{1} \ldots \pi_{r}}$ is the Levi-Civita permutation symbol. One can also show that as expected,

$$
(-1)^{\left|\pi^{\prime} \circ \pi\right|}=(-1)^{\left|\pi^{\prime}\right|+|\pi|}=(-1)^{\left|\pi^{\prime}\right|}(-1)^{|\pi|},
$$

for the composition of the permutation $\pi^{\prime}$ with the permutation $\pi$.

The ordered collection of vectors $v_{1}, \ldots, v_{r}, v_{i}=p_{i}-p_{0}$, defining a simplex, induces an orientation on the hyperplane containing it. It is easy to see that the collection of vectors $u_{1}, \ldots, u_{r}, u_{i}=p_{i}-p_{i-1}=v_{i}-v_{i-1}$, where we use the convention that $v_{0}=0$, has the same orientation. The orientation of the simplex is the orientation of either of these collections of vectors.

Transposing any two points $p_{i}$ and $p_{j}, i, j>0$, in the simplex $s=\left[p_{0}, p_{1}, \ldots, p_{r}\right]$ is equivalent to transposing the $v_{i}$ and $v_{j}$, hence, a transposition of the points will reverse the orientation. Similarly, it can be easily verified that a transposition of any point $p_{i}, i>0$ with $p_{0}$ reverses the orientation. Thus, any transposition of a pair of points defining a simplex will reverse its orientation.

Given a simplex $s$, the simplex containing the same set of points but having the opposite orientation is denoted by $-s$. Thus, identifying simplices containing identical vertices and having identical orientations we may write

$$
\left[p_{\pi_{0}}, \ldots, p_{\pi_{r}}\right]=(-1)^{|\pi|}\left[p_{0}, \ldots, p_{r}\right] .
$$

It is noted that Equation (2.9) still holds as the vectors $v_{i}$ and the vectors $u_{i}$ induce the same orientation on a simplex.

2.5. Simplices on the Boundaries and their Orientations. Given a simplex $s=\left[p_{0}, p_{1}, \ldots, p_{r}\right]$ of dimension $r$, a collection of $r+1$ simplices of dimension $r-1$ is obtained by omitting each time one of the vertices. Thus, denoting by a superimposed "hat" an omitted item, for each $i=0, \ldots, r$, the $(r-1)$-dimensional simplex $\tau_{i}$ is defined by omitting the $i$-th vertex as

$$
\tau_{i}=(-1)^{i}\left[p_{0}, \ldots, \hat{p}_{i}, \ldots, p_{r}\right] .
$$

The simplex $\tau_{i}$ is referred to as the $i$-th face of the simplex $s$.

In terms of the vectors $v_{i}=p_{i}-p_{0}$, we observe that $\tau_{0}$ is the simplex starting at $p_{1}$ and determined by $v_{i}-v_{1}$, so that

$$
\tau_{0}=\left[v_{2}-v_{1}, \ldots, v_{r}-v_{1}\right], \quad \text { and } \tau_{i}=(-1)^{i}\left[v_{1}, \ldots, \widehat{v}_{i}, \ldots, v_{r}\right], \quad i=1, \ldots, r \text {. }
$$

Using the vectors $u_{i}=p_{i}-p_{i-1}$, one has

$$
\begin{aligned}
\tau_{0} & =\left[u_{2}, \ldots, u_{r}\right], \\
\tau_{i} & =(-1)^{i}\left[u_{1}, \ldots, u_{i-1}, u_{i}+u_{i+1}, u_{i+2}, \ldots, u_{r}\right], \quad i=1, \ldots, r-1, \\
\tau_{r} & =(-1)^{r}\left[u_{1}, \ldots, u_{r-1}\right] .
\end{aligned}
$$

The collection of simplices $\tau_{0}, \ldots, \tau_{r}$ is referred to as the boundary of the simplex and it will be denoted by $\partial s$.

The factor $(-1)^{i}$ indicating the positive orientation of the face may be motivated as follows. Consider the $r$-simplex

$$
s_{i}=\left[p_{i}, p_{0}, \ldots, \widehat{p}_{i}, \ldots, p_{r}\right]=(-1)^{i}\left[p_{0}, \ldots, p_{r}\right] .
$$


It follows that $\left[p_{0}, \ldots, p_{r}\right]$ is positively oriented if and only if $s_{i}$ is. Note that the vector $p_{0}-p_{i}$ pointing from the vertex $p_{i}$ to the set containing the points of $\tau_{i}$ is directed "outwards" relative to the simplex $s$. If we add to $p_{0}-p_{i}$ an arbitrary vector in the plane of $\tau_{i}$ the same observation still holds. Hence, the orientation of the $(r-1)$-simplex $\left[p_{0}, \ldots, \widehat{p}_{i}, \ldots, p_{r}\right]$, which is determined by the orientation of $\left[u_{1}, \ldots, u_{i-1}, u_{i}+u_{i+1}, u_{i+2}, \ldots, u_{r}\right]$, is positive if $\left[v, u_{1}, \ldots, u_{i-1}, u_{i}+\right.$ $\left.u_{i+1}, u_{i+2}, \ldots, u_{r}\right]$ is a positively oriented $r$-simplex relative to $s$ for a vector $v$ that points in the outwards direction from the points of $\tau_{i}$. The orientation of $\tau_{i}$ is defined as to provide a consistent sense of "outwards" to points on the boundary of $s$. The same also applies to an arbitrary collection of $r-1$ vectors $w_{1}, \ldots, w_{r-1}$ on the plane of $\tau_{i}$.

2.6. Subdivisions. Simplices may be subdivided into simplices. A subdivision of an $r$-simplex $s$ is a collection of $r$-simplices $s_{1}, \ldots, s_{A}$ having the same orientation that cover $s$ whose interiors are disjoint and any two simplices intersect on faces (see [Kur72, p. 26I]). If $s_{1}, \ldots, s_{A}$ is a subdivision of $s$, we will write $s=s_{1}+\cdots+s_{A}$

The following example will be used below. Consider the simplex $s=\left[p_{0}, \ldots, p_{r}\right]$ and let

$$
q=\frac{1}{2}\left(p_{0}+p_{1}\right)=p_{0}+\frac{1}{2}\left(p_{1}-p_{0}\right)
$$

be the midpoint between $p_{0}$ and $p_{1}$. One may define the simplices $s_{1}=\left[p_{0}, q, p_{2}, \ldots, p_{r}\right]$ and $s_{2}=\left[q, p_{1}, \ldots, p_{r}\right]$. Thus, each point $y$ in $s_{1}$ is of the form

$$
y=y^{0} p_{0}+\frac{1}{2} y^{1}\left(p_{0}+p_{1}\right)+y^{2} p_{2}+\cdots+y^{r} p_{r}, \quad y^{i} \geqslant 0, \sum_{i=1}^{r} y^{i}=1 .
$$

However, rearranging the terms we have

$$
y=\left(y^{0}+\frac{1}{2} y^{1}\right) p_{0}+\frac{1}{2} y^{1} p_{1}+y^{2} p_{2}+\cdots+y^{r} p_{r},
$$

and setting $x^{0}=y^{0}+\frac{1}{2} y^{1}, x^{1}=\frac{1}{2} y^{1}, x^{2}=y^{2}, \ldots, x^{r}=y^{r}$, we observe that $\sum x^{i}=1, x^{i} \geqslant 0, x^{0} \geqslant x^{1}$ and $y=\sum_{i=0}^{r} x^{i} p_{i}$. Thus, each point in $s_{1}$ belongs also to $s$. Similarly, any $z \in s_{2}$ is of the form

$$
z=\frac{1}{2} z^{0}\left(p_{0}+p_{1}\right)+z^{1} p_{1}+z^{2} p_{2}+\cdots+z^{r} p_{r}, \quad z^{i} \geqslant 0, \sum_{i=1}^{r} z^{i}=1 .
$$

Rearranging the terms we have

$$
z=\frac{1}{2} z^{0} p_{0}+\left(\frac{1}{2} z^{0}+z^{1}\right) p_{1}+z^{2} p_{2}+\cdots+z^{r} p_{r},
$$

and setting $x^{0}=\frac{1}{2} z^{0}, x^{1}=\frac{1}{2} z^{0}+z^{1}, x^{2}=z^{2}, \ldots, x^{r}=z^{r}$, we observe that $\sum x^{i}=1$, $x^{i} \geqslant 0, x^{0} \leqslant x^{1}$ and $z=\sum_{i=0}^{r} x^{i} p_{i}$. Thus, each point in $s_{2}$ belongs to $s$, too.

In addition, any point $x=\sum x^{i} p_{i}$ in $s$ belongs to $s_{1}$ if $x^{0}>x^{1}$, it belongs to $s_{2}$ if $x^{0}<x^{1}$, and it belongs to both simplices if $x^{0}=x^{1}$. If $x^{0}=x^{1}$, then, $y^{0}=0, z^{1}=0$, and so $x$ belongs to the face $\left[q, p_{2}, \ldots, p_{r}\right]$ in $\partial s_{1}$ and to the face $(-1)^{1}\left[q, p_{2}, \ldots, p_{r}\right]$ of $\partial s_{2}$. We conclude that $s=s_{1}+s_{2}$.

It is also noted that if $u_{j}=p_{j}-p_{j-1}, j=1, \ldots, r$ are the vectors determining $s$, then

$$
s_{1}=\left[\frac{1}{2} u_{1}, \frac{1}{2} u_{1}+u_{2}, u_{3}, \ldots, u_{r}\right], \quad \text { and } \quad s_{2}=\left[\frac{1}{2} u_{1}, u_{2}, \ldots, u_{r}\right] .
$$


Clearly, the orientation of $s_{1}$ and $s_{2}$ are positive relative to $s$.

Using Equation (2.23) and denoting the faces of $s_{1}$ and $s_{2}$ by $\tau_{i}^{1}$ and $\tau_{i}^{2}$, respectively, we have

$$
\begin{aligned}
\tau_{0}^{1} & =\left[\frac{1}{2} u_{1}+u_{2}, u_{3}, \ldots, u_{r}\right], \\
\tau_{1}^{1} & =(-1)^{1}\left[u_{1}+u_{2}, u_{3}, \ldots, u_{r}\right], \\
\tau_{2}^{1} & =(-1)^{2}\left[\frac{1}{2} u_{1}, \frac{1}{2} u_{1}+u_{2}+u_{3}, u_{4}, \ldots, u_{r}\right], \\
\tau_{i}^{1} & =(-1)^{i}\left[\frac{1}{2} u_{1}, \frac{1}{2} u_{1}+u_{2}, u_{3}, \ldots, u_{i}+u_{i+1}, u_{i+2}, \ldots, u_{r}\right], 2<i<r, \\
\tau_{r}^{1} & =(-1)^{r}\left[\frac{1}{2} u_{1}, \frac{1}{2} u_{1}+u_{2}, u_{3}, \ldots, u_{r-1}\right],
\end{aligned}
$$

and

$$
\begin{aligned}
\tau_{0}^{2} & =\left[u_{2}, u_{3}, \ldots, u_{r}\right], \\
\tau_{1}^{2} & =(-1)^{1}\left[\frac{1}{2} u_{1}+u_{2}, u_{3}, \ldots, u_{r}\right], \\
\tau_{2}^{2} & =(-1)^{2}\left[\frac{1}{2} u_{1}, u_{2}+u_{3}, u_{4}, \ldots, u_{r}\right], \\
\tau_{i}^{2} & =(-1)^{i}\left[\frac{1}{2} u_{1}, u_{2}, u_{3}, \ldots, u_{i}+u_{i+1}, u_{i+2}, \ldots, u_{r}\right], 2<i<r, \\
\tau_{r}^{2} & =(-1)^{r}\left[\frac{1}{2} u_{1}, u_{2}, u_{3}, \ldots, u_{r-1}\right] .
\end{aligned}
$$

Using Equation (2.23) again, we finally arrive at

$$
\begin{aligned}
\tau_{0}^{1} & =-\tau_{1}^{2}, \\
\tau_{1}^{1} & =\tau_{1}, \\
\tau_{0}^{2} & =\tau_{0}, \\
\tau_{2}^{1}+\tau_{2}^{2} & =\tau_{2}, \\
\tau_{i}^{1}+\tau_{i}^{2} & =\tau_{i}, \quad i>2 .
\end{aligned}
$$

\section{Uniform Fluxes in Affine Spaces}

3.I. Basic Assumptions. We now lay down the basic postulates regarding uniform fluxes in affine spaces. By a uniform flux we mean a real valued function defined on the collection of all $(r-1)$-simplices in the $n$-dimensional affine space $\mathbf{A}$. For a uniform flux $T$ and an $(r-1)$-simplex $\tau$, the value $T(\tau)$ is may be interpreted as the total flux of a certain property that flows through the simplex $\tau$.

Temporarily, we suspend the convention exhibited by Equation (2.9) whereby a simplex $s=\left[p_{0}, \ldots, p_{r}\right]$ may be written as $s=\left[v_{1}, \ldots, v_{r}\right]$ or $s=\left[u_{1}, \ldots, u_{r}\right]$ and for the flux through the simplex $s$ we will write $T\left(\left[p_{0}, \ldots, p_{r}\right]\right)$.

Assumption 1: Translation Invariance. Consider $\tau=\left[p_{0}, \ldots, p_{r-1}\right]$, then, $T(\tau)$ is invariant under translation, i.e.,

$$
T\left(\left[p_{0}, \ldots, p_{r-1}\right]\right)=T\left(\left[p_{0}+w, \ldots, p_{r-1}+w\right]\right)
$$

for any vector $w \in \mathbf{V}$.

It is observed that $\tau=\left[p_{0}, \ldots, p_{r-1}\right]$ and $\tau+w=\left[p_{0}+w, \ldots, p_{r-1}+w\right]$ share the same vectors $u_{i}=p_{i}-p_{i-1}$ and $v_{i}=p_{i}-p_{0}$. Thus, given a function $\hat{T}$ of $r-1$ 
vectors, one may define $T\left(\left[p_{0}, \ldots, p_{r-1}\right]\right)=\hat{T}\left(p_{1}-p_{0}, p_{2}-p_{1}, \ldots, p_{r-1}-p_{r-2}\right)$ which is clearly invariant under translation. Conversely, we may write

$$
\begin{aligned}
T\left(\left[p_{0}, \ldots, p_{r-1}\right]\right) & =T\left(\left[p_{0}, p_{0}+u_{1}, \ldots, p_{0}+\sum u_{i}\right]\right) \\
& =T\left(\left[p_{0}+w, p_{0}+w+u_{1}, \ldots, p_{0}+w+\sum u_{i}\right]\right)
\end{aligned}
$$

and since the last expression is independent of $w, T\left(\left[p_{0}, \ldots, p_{r-1}\right]\right)$ depends only on $u_{1}, \ldots, u_{r-1}$ and may be represented by a function $\hat{T}$ as above. In the sequel we will omit the "hat" and will use $T\left(u_{1}, \ldots, u_{r-1}\right)$ to denote the corresponding value of the flux mapping.

Assumption 2: Homogeneity. We recall (see section 2.3) that one can fit $(r-1)$ ! simplices into an $(r-1)$-cube. Thus, if we multiply one of the vectors $u_{i}$ that determine the simplex by a positive number $a$, one side of the cube will be multiplied by the same number. It is natural therefore to assume that the flux will be multiplied by $a$ also, formally, that for all $i=1, \ldots, r-1$,

$$
T\left(u_{1}, \ldots, u_{i-1}, a u_{i}, u_{i+1}, \ldots, u_{r-1}\right)=a T\left(u_{1}, \ldots, u_{i-1}, u_{i}, u_{i+1}, \ldots, u_{r-1}\right) .
$$

We extend this assumption to the case $a=0$ : if $u_{i}=0$, one does not have any longer an $(r-1)$-simplex.

Let $s$ be an $r$-simplex in $\mathbf{A}$ whose boundary consists of the $(r-1)$-simplices $\tau_{i}$, $i=0, \ldots, r$. Basic properties of uniform fluxes are concerned with the total flux out of the various faces on the boundary of $s$, i.e.,

$$
T(\partial s):=\sum_{i=0}^{r} T\left(\tau_{i}\right) .
$$

Assumption 3: Additivity under subdivision. Let $\tau$ be an $(r-1)$-simplex and $\tau_{1}, \ldots, \tau_{A}$ be a subdivision of $\tau$. We assume that

$$
T(\tau)=\sum_{a=1}^{A} T\left(\tau_{a}\right) .
$$

Let $s$ be an $r$-simplex and let $s_{1}, \ldots, s_{B}$ be a subdivision of $s$. It is assumed that

$$
T(\partial s)=\sum_{b=1}^{B} T\left(\partial s_{b}\right) .
$$

This assumption, associated with the locality of the flux operator, states that the total flux out of $s$ is the sum of the fluxes out of its various parts.

We now apply the additivity property for $r$-simplices (3.5) to the subdivision $s_{1}, s_{2}$ of a simplex $s$ as in the example presented in Section 2.6. Using Equation 
(2.27) we obtain

$$
\begin{aligned}
T\left(\tau_{0}\right)+T\left(\tau_{1}\right)+\sum_{i=2}^{r} T\left(\tau_{r}\right)= & T\left(\tau_{0}^{1}\right)+T\left(\tau_{1}^{1}\right)+\sum_{i=2}^{r} T\left(\tau_{r}^{1}\right) \\
& +T\left(\tau_{0}^{2}\right)+T\left(\tau_{1}^{2}\right)+\sum_{i=2}^{r} T\left(\tau_{r}^{2}\right), \\
= & T\left(-\tau_{1}^{2}\right)+T\left(\tau_{1}\right)+T\left(\tau_{0}\right)+T\left(\tau_{1}^{2}\right)+\sum_{i=2}^{r} T\left(\tau_{r}\right),
\end{aligned}
$$

where we used the additivity property (3.4) to obtain the last line above. It follows immediately that

$$
T\left(\tau_{1}^{2}\right)=-T\left(-\tau_{1}^{2}\right) .
$$

Since the construction above may be used for any $(r-1)$-simplex $\tau$ with $\tau_{1}^{2}=\tau$, we conclude that for any $(r-1)$-simplex $\tau$,

$$
T(\tau)=-T(-\tau), .
$$

Next, we make use of the additivity assumption of Equation (3.4) for $(r-1)$ simplices and apply it to a subdivision $\tau=\tau_{1}+\tau_{2}, \tau=\left[p_{0}, \ldots, p_{r-1}\right], \tau_{1}=$ $\left[p_{0}, q, p_{2}, \ldots, p_{r-1}\right], \tau_{2}=\left[q, p_{1}, \ldots, p_{r-1}\right]$, as in Section 2.6 but pertaining to $(r-1)$-simplices. Keeping the same notation, the simplices are determined by the vectors $u_{i}, i=1, \ldots, r-1$. Thus, $T(\tau)=T\left(\tau_{1}\right)+T\left(\tau_{2}\right)$ implies that

$$
\begin{aligned}
T\left(u_{1}, \ldots, u_{r-1}\right) & =2 T\left(\frac{1}{2} u_{1}, u_{2}, \ldots, u_{r-1}\right) \\
= & T\left(\frac{1}{2} u_{1}, \frac{1}{2} u_{1}+u_{2}, u_{3}, \ldots, u_{r-1}\right)+T\left(\frac{1}{2} u_{1}, u_{2}, \ldots, u_{r-1}\right),
\end{aligned}
$$

where in the first line we used the homogeneity property and in the second line we used the subdivision (2.24) and the additivity property. Since $u_{1}$ is arbitrary, we obtain

$$
T\left(u_{1}, u_{1}+u_{2}, u_{3}, \ldots, u_{r-1}\right)=T\left(u_{1}, u_{2}, u_{3}, \ldots, u_{r-1}\right) .
$$

Since we can re-enumerate the points on the simplex, or equivalently, we can make a subdivision along a different vector, we conclude that

$$
T\left(u_{1}, \ldots, u_{i-1}, u_{i-1}+u_{i}, u_{i+1}, \ldots, u_{r-1}\right)=T\left(u_{1}, \ldots, u_{i-1}, u_{i}, u_{i+1}, \ldots, u_{r-1}\right) .
$$

In particular,

$$
T\left(u_{1}, \ldots, u_{i}, u_{i}, u_{i+2}, \ldots, u_{r-1}\right)=0 .
$$

Using homogeneity, we also have

$$
\begin{aligned}
T\left(u_{1}, \ldots, u_{i-1}, a u_{i-1}+u_{i}, u_{i+1}, \ldots, u_{r-1}\right) & =\frac{1}{a} T\left(u_{1}, \ldots, a u_{i-1}, a u_{i-1}+u_{i}, u_{i+1}, \ldots, u_{r-1}\right), \\
& =\frac{1}{a} T\left(u_{1}, \ldots, a u_{i-1}, u_{i}, u_{i+1}, \ldots, u_{r-1}\right),
\end{aligned}
$$


and it follows that

$T\left(u_{1}, \ldots, u_{i-1}, a u_{i-1}+u_{i}, u_{i+1}, \ldots, u_{r-1}\right)=T\left(u_{1}, \ldots, u_{i-1}, u_{i}, u_{i+1}, \ldots, u_{r-1}\right)$.

Next, consider the vectors $v_{i}=p_{i}-p_{0}=\sum_{j=1}^{i} u_{j}, i=1, \ldots, r-1$. Using Equation (3.II) repeatedly

$$
\begin{aligned}
T\left(v_{1}, \ldots, v_{r-1}\right) & =T\left(u_{1}, u_{1}+u_{2}, \ldots, \sum_{j=1}^{r-2} u_{j}, \sum_{j=1}^{r-1} u_{j}\right), \\
& =T\left(u_{1}, u_{1}+u_{2}, \ldots, \sum_{j=1}^{r-3} u_{j}, \sum_{j=1}^{r-2} u_{j}, u_{r-1}\right), \\
& =T\left(u_{1}, u_{1}+u_{2}, \ldots, \sum_{j=1}^{r-3} u_{j}, u_{r-2}, u_{r-1}\right)
\end{aligned}
$$

etc., and one concludes that

$$
T\left(v_{1}, \ldots, v_{r-1}\right)=T\left(u_{1}, \ldots, u_{r-1}\right) .
$$

Since $\tau_{\pi}=\left[p_{0}, p_{0}+v_{\pi_{1}}, \ldots, p_{0}+v_{\pi_{r-1}}\right]=(-1)^{|\pi|}\left[p_{0}, p_{0}+v_{1}, \ldots, p_{0}+v_{r-1}\right]$, Equations (3.8) and (3.16) give

$$
\begin{aligned}
& T\left(v_{\pi_{1}}, \ldots, v_{\pi_{r-1}}\right)=(-1)^{|\pi|} T\left(v_{1}, \ldots, v_{r-1}\right), \\
& T\left(u_{\pi_{1}}, \ldots, u_{\pi_{r-1}}\right)=(-1)^{|\pi|} T\left(u_{1}, \ldots, u_{r-1}\right),
\end{aligned}
$$

which makes the flux an alternating function of its arguments. It is noted that Equations (3.12) and (3.17) imply that the total flux vanishes if some of the vectors are recurring.

Remark 3.I. For the case where $r-1=n$, the assumptions above are sufficient to prove that the action of the flux mapping is additive. Consider for example the $n+1$ vectors $v_{1}^{\prime}, v_{1}, \ldots, v_{n}$ where we assume that $v_{1}, \ldots, v_{n}$ are linearly independent so that we may write $v_{1}^{\prime}=\sum_{i} a^{i} v_{i}$. Using Equation (3.I4) repeatedly, one has

$$
\begin{aligned}
T\left(v_{1}^{\prime}, v_{2}, \ldots, v_{n}\right) & =T\left(\sum_{i} a^{i} v_{i}, v_{2}, \ldots, v_{n}\right), \\
& =T\left(a^{1} v_{1}, v_{2}, \ldots, v_{n}\right), \\
& =a^{1} T\left(v_{1}, v_{2}, \ldots, v_{n}\right) \\
T\left(v_{1}+v_{1}^{\prime}, v_{2}, \ldots, v_{n}\right) & =T\left(v_{1}+\sum_{i} a^{i} v_{i}, v_{2}, \ldots, v_{n}\right), \\
& =T\left(v_{1}+a^{1} v_{1}, v_{2}, \ldots, v_{n}\right), \\
& =\left(1+a^{1}\right) T\left(v_{1}, v_{2}, \ldots, v_{n}\right),
\end{aligned}
$$


and so

$$
T\left(v_{1}+v_{1}^{\prime}, v_{2}, \ldots, v_{n}\right)=T\left(v_{1}, v_{2}, \ldots, v_{n}\right)+T\left(v_{1}^{\prime}, v_{2}, \ldots, v_{n}\right) .
$$

Assumption 4: Balance. Let $s$ be an $r$-simplex in A whose boundary consists of the $(r-1)$-simplices $\tau_{i}, i=0, \ldots, r$. It is assumed that

$$
T(\partial s):=\sum_{i=0}^{r} T\left(\tau_{i}\right)=0 .
$$

Thus, we assume that the sum of all fluxes on the various faces of a simplex vanishes.

Remark 3.2. It is noted that the assumption of balance as in Equation (3.2I) implies the additivity property of Equation (3.5). Thus, once balance is assumed, the additivity assumption of Equation (3.5) is redundant.

3.2. Balance and Linearity. In this section we prove the second basic property of uniform fluxes: the assumption of balance implies multi-linearity. The notation $\Pi^{\prime}$ will be used for the collection of all permutations $\pi$ of $r$-symbols such that $\pi^{-1}(1)<\pi^{-1}(2)$, i.e., $u_{1}$ precedes $u_{2}$ in the list of vectors. For each $\pi \in \Pi^{\prime}$, let $s_{\pi}=(-1)^{|\pi|}\left[u_{\pi_{1}}, \ldots, u_{\pi_{r}}\right]$ so that all the simplices $s_{\pi}$ are of the same orientation and their union is the prism $x^{1} \geqslant x^{2}$ as in Section 2.3. The assumption of balance implies using Equations (2.23) that

$$
\begin{aligned}
& 0=T\left(\partial s_{\pi}\right)=(-1)^{|\pi|}\left[T\left(u_{\pi_{2}}, \ldots, u_{\pi_{r}}\right)\right. \\
& \left.+\sum_{i=1}^{r-1}(-1)^{i} T\left(u_{\pi_{1}}, \ldots, u_{\pi_{i-1}}, u_{\pi_{i}}+u_{\pi_{r+1}}, u_{\pi_{i+2}}, \ldots, u_{\pi_{r}}\right)+(-1)^{r} T\left(u_{\pi_{1}}, \ldots, u_{\pi_{r-1}}\right)\right] .
\end{aligned}
$$

The sum of the equation above over all $\pi \in \Pi^{\prime}$ gives

$$
\begin{aligned}
0= & \sum_{\pi \in \Pi^{\prime}}(-1)^{|\pi|} T\left(u_{\pi_{2}}, \ldots, u_{\pi_{r}}\right)+\sum_{\pi \in \Pi^{\prime}}(-1)^{|\pi|}(-1)^{r} T\left(u_{\pi_{1}}, \ldots, u_{\pi_{r-1}}\right) \\
& +\sum_{\pi \in \Pi^{\prime}} \sum_{i=1}^{r-1}(-1)^{|\pi|}(-1)^{i} T\left(u_{\pi_{1}}, \ldots, u_{\pi_{i-1}}, u_{\pi_{i}}+u_{\pi_{i+1}}, u_{\pi_{i+2}}, \ldots, u_{\pi_{r}}\right) .
\end{aligned}
$$

Denoting the three terms on the right-hand side of the last equation by $t_{a}, t_{b}$, and $t_{c}$, respectively, it may be written in the form

$$
0=t_{a}+t_{b}+t_{c}
$$

Consider first the terms in the sum of $t_{a}$ for which $\pi_{1}=1$. Using the fact that $T$ is alternating, i.e., Equation (3.I7), one has

$$
\begin{aligned}
\sum_{\pi \in \Pi^{\prime}, \pi_{1}=1}(-1)^{|\pi|} T\left(u_{\pi_{2}}, \ldots, u_{\pi_{r}}\right) & =\sum_{\pi \in \Pi^{\prime}, \pi_{1}=1}(-1)^{2|\pi|} T\left(u_{2}, \ldots, u_{r}\right), \\
& =(r-1) ! T\left(u_{2}, \ldots, u_{r}\right)
\end{aligned}
$$


as the positions of $u_{2}, \ldots, u_{r}$ are not constrained by the requirement that $\pi^{-1}(1)<$ $\pi^{-1}(2)$.

Similarly, consider the terms in $t_{b}$ such that $\pi_{r}=2$. One has

$$
\begin{array}{rl}
\sum_{\pi \in \Pi^{\prime}, \pi_{r}=2}(-1)^{|\pi|}(-1)^{r} & T\left(u_{\pi_{1}}, \ldots, u_{\pi_{r-1}}\right) \\
& =\sum_{\pi \in \Pi^{\prime}, \pi_{r}=2}(-1)^{|\pi|+r}(-1)^{\left|\pi^{(2)}\right|} T\left(u_{1}, u_{3}, \ldots, u_{r}\right), \\
& =\sum_{\pi \in \Pi^{\prime}, \pi_{r}=2}(-1)^{2\left|\pi^{(2)}\right|+2 r-2} T\left(u_{1}, u_{3}, \ldots, u_{r}\right), \\
& =(r-1) ! T\left(u_{1}, u_{3}, \ldots, u_{r}\right) .
\end{array}
$$

Here, we used the fact that any permutation $\pi$ for which $\pi(r)=2$ may be factored in the form $\pi=\pi^{(2)} \circ \pi^{(1)}$, where $\pi^{(1)}:(1,2, \ldots, r) \mapsto(1,3, \ldots, r, 2)$, so that $\left|\pi^{(1)}\right|=r-2$, and $\pi^{(2)}:\{1,2, \ldots, r-1, r\} \rightarrow\left\{\pi_{1}, \pi_{2}, \ldots, \pi_{r-1}, r\right\}$, keeping the last term invariant. Hence, $|\pi|=\left|\pi^{(2)}\right|+\left|\pi^{(1)}\right|=\left|\pi^{(2)}\right|+r-2$. Again, the fact that $u_{2}$ is in the last position implies that there is no restriction on the positions of the other vectors.

Let $\Pi_{1} \subset \Pi^{\prime}$ be the collection of permutations not included in (3.25), i.e., permutations $\pi$ for which $\pi^{-1}(1)<\pi^{-1}(2)$ and $\pi_{1} \neq 1$. Similarly, let $\Pi_{2} \subset \Pi^{\prime}$ be the collection of permutations not included in (3.26), i.e., permutations $\pi$ for which $\pi^{-1}(1)<\pi^{-1}(2)$ and $\pi_{r} \neq 2$. We now consider the sum of terms

$$
t_{a}^{\prime}+t_{b}^{\prime}:=\sum_{\pi \in \Pi_{1}}(-1)^{|\pi|} T\left(u_{\pi_{2}}, \ldots, u_{\pi_{r}}\right)+\sum_{\pi^{\prime} \in \Pi_{2}}(-1)^{\left|\pi^{\prime}\right|+r} T\left(u_{\pi_{1}^{\prime}}, \ldots, u_{\pi_{r-1}^{\prime}}\right)
$$

in $t_{a}$ and $t_{b}$ not included above. Since $\pi_{1} \neq 1$, both $u_{1}$ and $u_{2}$ must appear in each element of $\Pi_{1}$ and as $\pi_{r} \neq 2$ the two vectors must appear in each element of $\Pi_{2}$. Consider the permutation $\rho:(1, \ldots, r) \mapsto(2, \ldots, r, 1)$. For each permutation $\pi=\left\{\pi_{1}, \ldots, \pi_{r}\right\}$, set $\pi^{\prime}=\rho \circ \pi:(1, \ldots, r) \mapsto\left(\pi_{2}, \ldots, \pi_{r}, \pi_{1}\right)$, such that $\left|\pi^{\prime}\right|=$ $|\rho \circ \pi|=|\pi|+r-1$. It is noted that $\pi^{\prime}=\rho \circ \pi$ satisfies the condition $\pi^{\prime-1}(1)<$ $\pi^{\prime-1}(2)$ and so composition with $\rho$ on the left, $\pi \mapsto \rho \circ \pi$, is a bijection of $\Pi_{1}$ onto $\Pi_{2}$. Hence,

$$
\begin{aligned}
t_{a}^{\prime}+t_{b}^{\prime} & =\sum_{\pi \in \Pi_{1}}\left[(-1)^{|\pi|} T\left(u_{\pi_{2}}, \ldots, u_{\pi_{r}}\right)+(-1)^{|\rho \circ \pi|+r} T\left(u_{(\rho \circ \pi)_{1}}, \ldots, u_{(\rho \circ \pi)_{r-1}}\right)\right], \\
& =\sum_{\pi \in \Pi_{1}}\left[(-1)^{|\pi|} T\left(u_{\pi_{2}}, \ldots, u_{\pi_{r}}\right)+(-1)^{|\pi|+2 r-1} T\left(u_{\pi_{2}}, \ldots, u_{\pi_{r}}\right)\right], \\
& =0 .
\end{aligned}
$$

As a result

$$
t_{a}+t_{b}=(r-1) !\left[T\left(u_{2}, \ldots, u_{r}\right)+T\left(u_{1}, u_{3}, \ldots, u_{r}\right)\right] .
$$




$$
\begin{aligned}
t_{c} & =\sum_{\pi \in \Pi^{\prime}} \sum_{i=1}^{r-1}(-1)^{|\pi|}(-1)^{i} T\left(u_{\pi_{1}}, \ldots, u_{\pi_{i-1}}, u_{\pi_{i}}+u_{\pi_{i+1}}, u_{\pi_{i+2}}, \ldots, u_{\pi_{r}}\right), \\
& =\sum_{i=1}^{r-1}(-1)^{i} t_{c i},
\end{aligned}
$$

where,

$$
t_{c i}=\sum_{\pi \in \Pi^{\prime}}(-1)^{|\pi|} T\left(u_{\pi_{1}}, \ldots, u_{\pi_{i-1}}, u_{\pi_{i}}+u_{\pi_{i+1}}, u_{\pi_{i+2}}, \ldots, u_{\pi_{r}}\right) .
$$

For a fixed value of $i$, let

$$
\rho_{i}:(1, \ldots, i-1, i, i+1, i+2, \ldots, r) \longmapsto(1, \ldots, i-1, i+1, i, i+2, \ldots, r)
$$

be the permutation that swaps the positions of $i$ and $i+1$. The permutation $\rho_{i}$ acts on $\Pi^{\prime}$ by composition $\pi \mapsto \rho_{i} \circ \pi$ with $(-1)^{\left|\rho_{i} \circ \pi\right|}=(-1)^{|\pi|+1}$. In addition, $\rho_{i}$ acts as an automorphism on the subset of permutations $\Pi_{3 i} \subset \Pi^{\prime}$ containing permutations for which we exclude $\pi_{i}=1$ and $\pi_{i+1}=2$ at the same time. That is, let $\Pi_{4 i}=\left\{\pi \in \Pi^{\prime} \mid \pi_{i}=1, \pi_{i+1}=2\right\}$, then, $\Pi_{3 i}=\Pi^{\prime} \backslash \Pi_{4 i}$. Setting

$$
t_{c i}^{\prime}=\sum_{\pi \in \Pi_{3 i}}(-1)^{|\pi|} T\left(u_{\pi_{1}}, \ldots, u_{\pi_{i-1}}, u_{\pi_{i}}+u_{\pi_{i+1}}, u_{\pi_{i+2}}, \ldots, u_{\pi_{r}}\right),
$$

one obtains

$$
\begin{aligned}
t_{c i}^{\prime}= & \sum_{\pi \in \Pi_{3 i}}(-1)^{|\pi|} T\left(u_{\pi_{1}}, \ldots, u_{\pi_{i-1}}, u_{\pi_{i}}+u_{\pi_{i+1}}, u_{\pi_{i+2}}, \ldots, u_{\pi_{r}}\right), \\
=\frac{1}{2}\left[\sum_{\pi \in \Pi_{3 i}}(-1)^{|\pi|} T\left(u_{\pi_{1}}, \ldots, u_{\pi_{i}}+u_{\pi_{i+1}}, \ldots, u_{\pi_{r}}\right)\right. & \\
& \left.\quad+\sum_{\pi \in \Pi_{3 i}}(-1)^{\left|\rho_{i} \circ \pi\right|} T\left(u_{\left(\rho_{i} \circ \pi\right)_{1}}, \ldots, u_{\left(\rho_{i} \circ \pi\right)_{i}}+u_{\left(\rho_{i} \circ \pi\right)_{i+1}}, \ldots, u_{\left(\rho_{i} \circ \pi\right)_{r}}\right)\right], \\
=\frac{1}{2} & {\left[\sum_{\pi \in \Pi_{3 i}}(-1)^{|\pi|} T\left(u_{\pi_{1}}, \ldots, u_{\pi_{i}}+u_{\pi_{i+1}}, \ldots, u_{\pi_{r}}\right)\right.} \\
\quad & \left.\quad+\sum_{\pi \in \Pi_{3 i}}(-1)^{|\pi|+1} T\left(u_{\pi_{1}}, \ldots, u_{\pi_{i}}+u_{\pi_{i+1}}, \ldots, u_{\pi_{r}}\right)\right], \\
=0 . &
\end{aligned}
$$


Thus,

$$
\begin{aligned}
t_{c i} & =\sum_{\pi \in \Pi_{4 i}}(-1)^{|\pi|} T\left(u_{\pi_{1}}, \ldots, u_{\pi_{i-1}}, u_{\pi_{i}}+u_{\pi_{i+1}}, u_{\pi_{i+2}}, \ldots, u_{\pi_{r}}\right), \\
& =\sum_{\pi \in \Pi_{4 i}}(-1)^{|\pi|+i-1} T\left(u_{1}+u_{2}, u_{\pi_{1}}, \ldots, u_{\pi_{i-1}}, u_{\pi_{i+2}}, \ldots, u_{\pi_{r}}\right), \\
& =\sum_{\pi \in \Pi_{4 i}}(-1)^{|\pi|+i-1+|\rho|} T\left(u_{1}+u_{2}, u_{3}, \ldots, u_{r}\right),
\end{aligned}
$$

where $\rho$ is a permutation of the form

$$
\rho:(1,2,3, \ldots, r) \longmapsto\left(1,2, \rho_{3}, \ldots, \rho_{r}\right) .
$$

Let $\eta_{i}$ be the permutation

$$
\eta_{i}:(1,2,3, \ldots, r) \longmapsto(3,4, \ldots, i-1,1,2, i+2, \ldots, r)
$$

so that for each $\pi$ there is a unique $\rho$ with $\pi=\eta_{i} \circ \rho$. Since $\left|\eta_{i}\right|$ is even, $(-1)^{|\rho|}=$ $(-1)^{|\pi|}$. We conclude that

$$
t_{c i}=\sum_{\pi \in \Pi_{4 i}}(-1)^{2|\pi|+i-1} T\left(u_{1}+u_{2}, u_{3}, \ldots, u_{r}\right)
$$

and

$$
\begin{aligned}
t_{c} & =\sum_{i=1}^{r-1}(-1)^{i} t_{c i}, \\
& =\sum_{i=1}^{r-1}(-1)^{i}\left[\sum_{\pi \in \Pi_{4 i}}(-1)^{2|\pi|+i-1} T\left(u_{1}+u_{2}, u_{3}, \ldots, u_{r}\right)\right], \\
& =-\sum_{i=1}^{r-1}\left[\sum_{\pi \in \Pi_{4 i}} T\left(u_{1}+u_{2}, u_{3}, \ldots, u_{r}\right)\right]
\end{aligned}
$$

Using the fact that there are $(r-2)$ ! permutations in $\Pi_{4 i}$ for each of the $r-1$ values of $i$ we obtain

$$
t_{c}=-(r-1) ! T\left(u_{1}+u_{2}, u_{3}, \ldots, u_{r}\right) .
$$

Combining Equations (3.29), (3.39) into the balance (3.24), one arrives at

$$
T\left(u_{1}+u_{2}, u_{3}, \ldots, u_{r}\right)=T\left(u_{1}, u_{3}, \ldots, u_{r}\right)+T\left(u_{2}, u_{3}, \ldots, u_{r}\right) .
$$

since $T$ is alternating by Equation (3.I7), for each $i=1, \ldots, r$,

$T\left(u_{1}, \ldots, u_{i}+u_{i}^{\prime}, \ldots, u_{r-1}\right)=T\left(u_{1}, \ldots, u_{i}, \ldots, u_{r-1}\right)+T\left(u_{1}, \ldots, u_{i}^{\prime}, \ldots, u_{r-1}\right)$.

It is noted that originally, each of the terms in the equations above was multiplied by $(r-1)$ ! the reason being the fact that we computed the total flux for the cubes on the faces of the prism and there are $(r-1)$ ! simplices in each such $(r-1)$-cube. 
3.3. Implications of Skew Symmetry and Multi-Linearity. Equations (3.4I) and (3.2) imply immediately

$$
T\left(u_{1}, \ldots, u_{i}, \ldots, u_{r-1}\right)+T\left(u_{1}, \ldots,-u_{i}, \ldots, u_{r-1}\right)=T\left(u_{1}, \ldots, 0, \ldots, u_{r-1}\right)=0
$$

and so we obtain

$$
T\left(u_{1}, \ldots,-u_{i}, \ldots, u_{r-1}\right)=-T\left(u_{1}, \ldots, u_{i}, \ldots, u_{r-1}\right) .
$$

It follows that the homogeneity property (3.2) applies also to the case $a<0$. We have obtained that the flux operator is both additive and homogeneous in each of its arguments and we conclude that

$$
T: \mathbf{V}^{r-1} \longrightarrow \mathbb{R}
$$

is an alternating multilinear mapping.

Next, we show that the properties we obtained above imply that the flux vanishes in the case where the vectors $u_{1}, \ldots, u_{r-1}$ are linearly dependent. This is of course expected as linear dependence implies that the simplex collapses to a hyperplane of a lower dimension. If the vectors are not linearly independent, one of the vectors, say $u_{k}$ may be represented as a linear combination of the others in the form $u_{k}=$ $\sum_{j} a_{j} u_{j}$ where $j=1, \ldots, \hat{k}, \ldots, r-1$. Thus,

$$
\begin{aligned}
T\left(u_{1}, \ldots, u_{r-1}\right) & =T\left(u_{1}, \ldots, u_{k-1}, \sum_{j} a_{j} u_{j}, u_{k+1}, \ldots, u_{r-1}\right), \\
& =\sum_{j} a_{j} T\left(u_{1}, \ldots, u_{k-1}, u_{j}, u_{k+1}, \ldots, u_{r-1}\right), \\
& =0
\end{aligned}
$$

because each term in the sum contains $u_{j}$ twice (see Equation (3.I2)). Conversely, a multilinear map that vanishes for recurring vectors is necessarily alternating;

$$
\begin{aligned}
0= & T\left(u_{1}, \ldots, u_{i}+u_{i}^{\prime}, \ldots, u_{i}+u_{i}^{\prime}, \ldots u_{r-1}\right), \\
= & T\left(u_{1}, \ldots, u_{i}, \ldots, u_{i}, \ldots u_{r-1}\right)+T\left(u_{1}, \ldots, u_{i}^{\prime}, \ldots, u_{i}^{\prime}, \ldots u_{r-1}\right) \\
& \quad+T\left(u_{1}, \ldots, u_{i}, \ldots, u_{i}^{\prime}, \ldots u_{r-1}\right)+T\left(u_{1}, \ldots, u_{i}^{\prime}, \ldots, u_{i}, \ldots u_{r-1}\right),
\end{aligned}
$$

implies

$$
T\left(u_{1}, \ldots, u_{i}^{\prime}, \ldots, u_{i}, \ldots u_{r-1}\right)=-T\left(u_{1}, \ldots, u_{i}, \ldots, u_{i}^{\prime}, \ldots u_{r-1}\right) .
$$

3.4. Algebraic Cauchy Theorem. The results of the foregoing sections may be summarized as follows. Let $T$ be a function of $(r-1)$-simplices satisfying Assumptions I -4 of Section 3.I, then, $T(\tau)$ depends on the vectors $\left(v_{1}, \ldots, v_{r-1}\right)$ determining $\tau$ multi-linearly and skew-symmetrically. Thus, there is an alternating $(r-1)$ tensor $J \in \otimes^{r-1}\left(\mathbf{V}^{*}\right)$, where $\mathbf{V}$ is the translation space of the affine space $\mathbf{A}$, such that

$$
T(\tau)=J\left(v_{1}, \ldots, v_{r-1}\right) .
$$

We will refer to this conclusion as the algebraic Cauchy theorem. 
The classical algebraic Cauchy theorem applies in the case where the space $\mathbf{A}$ is a 3-dimensional Euclidean space so that the vector space $\mathbf{V}$ has an inner product structure. It states that if $\tau$ is determined by the vectors $v$ and $u$, then,

$$
T(\tau)=(\mathbf{J} \cdot \mathbf{n}) A,
$$

where $\mathbf{J}$ is a vector, $\mathbf{n}$ is the unit normal to the simplex pointing in a direction so that $(\mathbf{n}, v, u)$ are positively oriented (i.e., generate a right-handed triad), and $A$ is the area of the simplex. Thus, one has $\mathbf{n} A=\frac{1}{2} v \times u$ where $\times$ denotes the vectorproduct of the two vectors in the 3 -dimensional Euclidean space. Thus,

$$
T(\tau)=\frac{1}{2} \mathbf{J} \cdot(v \times u),
$$

which is clearly bi-linear, and alternating in the arguments $v$ and $u$. For more details on the comparison of the classical Cauchy formula and the metric independent version described above, see Example 4.4.

\section{EXTERIOR Algebra}

Once fluxes are shown to be representable by alternating multilinear mappings, we present some of their properties. In addition, we study the properties of simplices that influence the total fluxes through them-their extents. These are represented mathematically by multivectors. While somewhat disguised below, multivectors are introduced as elements of the dual space to the space of fluxes-the space of multilinear mappings. Since the space of multilinear mappings is finite dimensional, this is equivalent to the introduction of multilinear mappings as elements of the dual to the space of multivectors.

4.I. Multivectors. We will roughly refer to the collection of properties of an $r$ simplex $s$ that determine the total flux through it, for an arbitrary flux, as the extent of the simplex. Mathematically, the extent of a simplex is represented by the multivector associated with with $s$ and is denoted by $\{s\}$. Thus, for two simplices $s$ and $s^{\prime}$ we write $\{s\}=\left\{s^{\prime}\right\}$ if $T(s)=T\left(s^{\prime}\right)$ for every flux $T$. It is natural therefore to extend the notation, and write $T(\{s\})$ for the total flux associated with the multivector. Formally, a multivector may be introduced as an equivalence class of simplices under the equivalence relation $s \sim s^{\prime}$ if $T(s)=T\left(s^{\prime}\right)$ for every flux $T$.

From the assumption of translation invariance, $T(s)$ depends only on the vectors $v_{i}=p_{i}-p_{0}$ or equivalently, see (3.I6), on the vectors $u_{i}=p_{i}-p_{i-1}, i=1, \ldots r$. It follows that $\{s\}$ is determined uniquely by these vectors. Given vectors $v_{i}$ (or $u_{i}$ ) as above, we denote the resulting multivector by $v_{1} \wedge \cdots \wedge v_{r}$ (respectively, $u_{1} \wedge \cdots \wedge u_{r}$ ). Thus, $\{s\}=v_{1} \wedge \cdots \wedge v_{r}$ and

$$
T\left(v_{1}, \ldots, v_{r}\right)=T\{s\}=T\left(v_{1} \wedge \cdots \wedge v_{r}\right) .
$$

From the properties of fluxes, one immediately obtains

$u_{1} \wedge \cdots \wedge a u_{i} \wedge \cdots \wedge u_{j} \wedge \cdots \wedge u_{r}=u_{1} \wedge \cdots \wedge u_{i} \wedge \cdots \wedge a u_{j} \wedge \cdots \wedge u_{r}, \quad$ for all $i, j=1, \ldots, r$.

Thus, we write any of the expressions above as $a\left(u_{1} \wedge \cdots \wedge u_{r}\right)$ so that

$a\left(u_{1} \wedge \cdots \wedge u_{r}\right)=u_{1} \wedge \cdots \wedge a u_{i} \wedge \cdots \wedge u_{j} \wedge \cdots \wedge u_{r}=u_{1} \wedge \cdots \wedge u_{i} \wedge \cdots \wedge a u_{j} \wedge \cdots \wedge u_{r}$, 
and clearly,

$$
a\left(u_{1} \wedge \cdots \wedge u_{r}\right)+b\left(u_{1} \wedge \cdots \wedge u_{r}\right)=(a+b)\left(u_{1} \wedge \cdots \wedge u_{r}\right) .
$$

Furthermore, it follows form the fact that the flux mapping is alternating, i.e., Equation (3.17), that

$$
u_{\pi_{1}} \wedge \cdots \wedge u_{\pi_{r}}=(-1)^{|\pi|} u_{1} \wedge \cdots \wedge u_{r}=\varepsilon_{\pi_{1} \ldots \pi_{r}} u_{1} \wedge \cdots \wedge u_{r} .
$$

One can define naturally the zero multivector to be the multivector for which $T(0)=0$ for every flux $T$. From (3.45) it follows that for $r$ nonvanishing vectors $v_{1}, \ldots, v_{r}, v_{1} \wedge \cdots \wedge v_{r} \neq 0$ implies that the vectors are linearly independent. Conversely, using component representation, one can show that if the vectors are linearly independent, a flux $T$ may be constructed such that $T\left(v_{1} \wedge \cdots \wedge v_{r}\right) \neq 0$. Thus,

$$
v_{1} \wedge \cdots \wedge v_{r} \neq 0
$$

if and only if the vectors are linearly independent.

In an analogous manner, we set

$$
w_{1} \wedge \cdots \wedge w_{r}=u_{1} \wedge \cdots \wedge u_{r}+v_{1} \wedge \cdots \wedge v_{r}
$$

if

$$
T\left(w_{1} \wedge \cdots \wedge w_{r}\right)=T\left(u_{1} \wedge \cdots \wedge u_{r}\right)+T\left(v_{1} \wedge \cdots \wedge v_{r}\right)
$$

for every flux $T$. In particular, the additivity property (3.4I) implies that

$$
u_{1} \wedge \cdots \wedge\left(u_{i}+u_{i}^{\prime}\right) \wedge \cdots \wedge u_{r-1}=u_{1} \wedge \cdots \wedge u_{i} \wedge \cdots \wedge u_{r-1}+u_{1} \wedge \cdots \wedge u_{i}^{\prime} \wedge \cdots \wedge u_{r-1} .
$$

Next, we wish to extend the definition of a multivector so that it represents the extent of geometrical objects more general than simplices.

4.2. Chains. A polyhedral $r$-chain in the affine space $\mathbf{A}$ is a formal linear combination of $r$-simplices, that is, an expression of the form

$$
B=\sum_{i=1}^{q} a_{i} s_{i},
$$

where $q$ is some finite integer. Chains may be thought of as simple functions defined over $r$-dimensional domains in $\mathbf{A}$. Choose a specific orientation for each of the planes containing simplices belonging to the collection $s_{1}, \ldots, s_{q}$. Assume first that the interior of the simplices $s_{i}$ are disjoint. Then, on the domain consisting of the union of the various simplices, the function $B$ is defined by $B(x)=a_{i}$ if $x \in s_{i}$ and $s_{i}$ is positively oriented; $B(x)=-a_{i}$ if $x \in s_{i}$ and $s_{i}$ is negatively oriented relative to the orientation given to the plane of $s_{i} ; B(x)$ vanishes otherwise. From this point of view, and ignoring incompatibilities on the faces of the $r$-simplices, it is clear that further subdivision of the simplices will not change the function and hence it results in the same $r$-chain. If the interiors of the simplices in (4.9) are not disjoint, by further subdivision one can obtain a linear combination in which the various simplices have disjoint interiors. (More formally, e.g., [Mun84], a chain may be defined as a function on the space of simplices that vanishes on all but a finite number of simplices.) Polyhedral chains may be added and may be multiplied by real numbers by performing these operations on the corresponding functions. 
Thus for example, if $A=B+B^{\prime}$, then, $A(x)=B(x)+B^{\prime}(x)$ for all $x$ in the affine space A. In Sections 3.I-3.2 we used examples of chains in the proofs. In particular, the boundary of an $r$-simplex may be regarded as a chain

$$
\partial s=\sum_{i=0}^{r} \tau_{i} .
$$

4.3. Chains and Multivectors. Extending the representation of the extent of a simplex $s$ by the multivector $\{s\}$, one can now represent the extent of an $r$-chain $B=\sum_{i} a_{i} s_{i}$ by an $r$-multivector written formally as $\{B\}=\sum_{i} a_{i}\left\{s_{i}\right\}$. Naturally, the action of a flux $T$ is now extended to chains by

$$
T(B)=T(\{B\})=T\left(\sum_{i} a_{i}\left\{s_{i}\right\}\right)=\sum_{i} a_{i} T\left(\left\{s_{i}\right\}\right) .
$$

By the balance assumption (3.21),

$$
T(\{\partial s\})=\sum_{i=0}^{r} T\left\{\tau_{i}\right\}=0
$$

for any flux $T$. Hence, as an $r$-multivector,

$$
\{\partial s\}=0 .
$$

It is noted that the chain $\partial s$ is not the zero chain but the multivector associated with is vanishes. This is the metric free analog of the identity $\sum_{i=0}^{3} A_{i} \mathbf{n}^{i}=0$ for a tetrahedron in the 3 -dimensional Euclidean space, where $A_{i}$ is the area of the $i$-th face and $\mathbf{n}^{i}$ is the unit normal to the $i$-th face.

Together with (3.2), we conclude that $T$, which is an alternating multilinear functions of vectors may be regarded as a linear function of multivectors. (Strictly speaking, one should make a distinction between the multilinear function of vectors and the linear function of multivectors.) The algebraic Cauchy theorem as presented here may be viewed as an analog to Whitney's theorem on an algebraic criterion for a multivector (see [Whis7, pp. I65-I67]). Here, we used only elementary notions and arguments in order to motivate the use of alternating tensors.

4.4. Component Representation. Let $\left\{e_{i}\right\}_{i=1}^{n}$ be a basis of $\mathbf{V}$ and let $\left\{e^{i}\right\}_{i=1}^{n}$ be the corresponding dual basis of the dual space, defined by the condition $e^{i}\left(e_{j}\right)=\delta_{j}^{i}$, where $\delta_{j}^{i}$ is the Kronecker symbol. One has $e^{i}(v)=e^{i}\left(\sum_{j} v^{j} e_{j}\right)=v^{i}$, and given a covector $\phi \in \mathbf{V}^{*}$, the components of $\phi$ relative to $\left\{e^{i}\right\}$ are given by $\phi_{i}=\phi\left(e_{i}\right)$. The array of a multilinear function $T: \mathbf{V}^{r} \rightarrow \mathbb{R}$ is given by $T_{i_{1} \ldots i_{r}}=T\left(e_{i_{1}}, \ldots, e_{i_{r}}\right)$. With

$$
\begin{aligned}
e^{i_{1}} \otimes \cdots \otimes e^{i_{r}}\left(v_{1}, \ldots, v_{r}\right) & =e^{i_{1}} \otimes \cdots \otimes e^{i_{r}}\left(\sum_{k_{1}} v_{1}^{k_{1}} e_{k_{1}}, \ldots, \sum_{k_{r}} v_{r}^{k_{r}} e_{k_{r}}\right), \\
& =v_{1}^{i_{1}} \cdots v_{r}^{i_{r}},
\end{aligned}
$$

one has $T=\sum_{i_{1}, \ldots, i_{r}} T_{i_{1} \ldots i_{r}} e^{i_{1}} \otimes \cdots \otimes e^{i_{r}}$ and $T\left(v_{1}, \ldots, v_{r}\right)=\sum_{i_{1}, \ldots, i_{r}} T_{i_{1} \ldots i_{r}} v_{1}^{i_{1}} \cdots v_{r}^{i_{r}}$.

One can write the equations above using multi-indices. For a multi-index $I=$ $\left(i_{1}, \ldots, i_{r}\right)$, with $|I|=r$, we write $T_{I}=T_{i_{1} \ldots i_{r}}$, so that $T=\sum_{I} T_{I} e^{i_{1}} \otimes \cdots \otimes e^{i_{r}}$. 
(We do not write $e^{I}=e^{i_{1}} \otimes \cdots \otimes e^{i_{r}}$ because we reserve the notation $e^{I}$ for another object.)

Next, we consider the consequences of the fact that a flux is an alternating multilinear function. Since $T$ vanishes on a collection of linearly dependent vectors, in case an index $j$ appears twice,

$$
T_{i_{1} \ldots j \ldots j \ldots i_{r}}=T\left(e_{i_{1}}, \ldots, e_{j}, \ldots, e_{j}, \ldots e_{i_{r}}\right)=0 .
$$

In addition, for any permutation $\pi:\{1, \ldots, r\} \rightarrow\{1, \ldots, r\}$,

$$
\begin{aligned}
T_{(I \circ \pi)_{1} \ldots(I \circ \pi)_{r}} & =T\left(e_{(I \circ \pi)_{1}}, \ldots, e_{(I \circ \pi)_{r}}\right), \\
& =(-1)^{|\pi|} T\left(e_{i_{1}}, \ldots, e_{i_{r}}\right), \\
& =(-1)^{|\pi|} T_{i_{1} \ldots i_{r}},
\end{aligned}
$$

so,

$$
T_{I \circ \pi}=(-1)^{|\pi|} T_{I} .
$$

Let $\gamma:\{1, \ldots, r\} \rightarrow\{1, \ldots, n\}$ be a monotone increasing injection regarded as a multi-index, i.e., $\gamma_{i}=\gamma(i)>\gamma_{j}=\gamma(j)$ if $i>j$. When we want to indicate that a multi-index $\gamma$ is increasing we will write $(\gamma)$. It follows that the array of $T$ is completely determined by the components for the increasing multi-indices. Since for any $I$ there is a unique increasing $\gamma$ containing the same elements, the multilinear mapping can now be expressed by

$$
\begin{aligned}
T & =\sum_{I} T_{I} e^{i_{1}} \otimes \cdots \otimes e^{i_{r}}, \\
& =\sum_{I,(\gamma)} \varepsilon_{I}^{\gamma} T_{\gamma} e^{i_{1}} \otimes \cdots \otimes e^{i_{r}},
\end{aligned}
$$

and we obtain

$$
T=\sum_{(\gamma)} T_{\gamma} e^{\gamma}, \quad \text { where } \quad e^{\gamma}=\sum_{I} \varepsilon_{I}^{\gamma} e^{i_{1}} \otimes \cdots \otimes e^{i_{r}} .
$$

It is observed that

$$
\begin{aligned}
e^{\gamma}\left(e_{i_{1}}, \ldots, e_{i_{r}}\right)=e^{\gamma}\left(e_{i_{1}} \wedge \cdots \wedge e_{i_{r}}\right) & =\sum_{J} \varepsilon_{J}^{\gamma} e^{j_{1}} \otimes \cdots \otimes e^{j_{r}}\left(e_{i_{1}}, \ldots, e_{i_{r}}\right), \\
& =\sum_{J} \varepsilon_{J}^{\gamma} \delta_{i_{1}}^{j_{1}} \cdots \delta_{i_{r}}^{j_{r}}, \\
& =\varepsilon_{I}^{\gamma},
\end{aligned}
$$

so that $e^{\gamma}$ is an alternating tensor which we also denote by

$$
e^{\gamma}=e^{\gamma_{1}} \wedge \cdots \wedge e^{\gamma_{r}} .
$$

Note that the definition of $e^{\gamma}$ in (4.I9) applies also for the case where the multiindex is not increasing and that it implies that

$$
e^{\gamma \circ \pi}=(-1)^{|\pi|} e^{\gamma}, \quad e^{(\gamma \circ \pi)_{1}} \wedge \cdots \wedge e^{(\gamma \circ \pi)_{r}}=(-1)^{|\pi|} e^{\gamma_{1}} \wedge \cdots \wedge e^{\gamma_{r}} .
$$

In fact, we may also consider $e^{I}$ where the multi-index $I$ need not even be an injection. We just set formally $e^{I}=0$ if $I$ contains two or more identical indices. 
In view of the skew symmetry property as in Equation (4.22), and observing that an injective multi-index $I$ may be written in the form $I=\gamma \circ \pi$ for an increasing multi-index $\gamma:\{1, \ldots, r\} \rightarrow\{1, \ldots, n\}$ and a permutation $\pi$ of $r$ symbols, we have $e^{I}=\sum_{(\gamma)} \varepsilon_{\gamma_{1} \ldots \gamma_{r}}^{i_{1} \ldots i_{r}} e^{\gamma}$, where the sum contains only one nonvanishing term. Thus,

$$
\begin{aligned}
\sum_{I} T_{I} e^{I} & =\sum_{I,(\gamma)} T_{I} \varepsilon_{\gamma_{1} \ldots \gamma_{r}}^{i_{1} \ldots i_{r}} e^{\gamma}, \\
& =\sum_{(\gamma)}\left(\sum_{I} T_{I} \varepsilon_{\gamma_{1} \ldots \gamma_{r}}^{i_{1} \ldots i_{r}}\right) e^{\gamma}, \\
& =\sum_{(\gamma)} r ! T_{\gamma} e^{\gamma},
\end{aligned}
$$

where in the last line we used the fact that both the Levi-Civita symbol and the array $T_{I}$ are skew symmetric (as in (4.I7)). Thus, Equation (4.I9) may be rewritten in the form

$$
T=\frac{1}{r !} \sum_{I} T_{I} e^{i_{1}} \wedge \cdots \wedge e^{i_{r}}
$$

To show that the collection of mappings $\left\{e^{\gamma}\right\}$, for increasing multi-indices $\gamma$, forms a basis for the space of $r$-multilinear mappings it remains to prove that they are linearly independent. If $\sum_{(\gamma)} T_{\gamma} e^{\gamma}=0$, then for every multi-index $I$,

$$
\begin{aligned}
0 & =\sum_{(\gamma)} T_{\gamma} e^{\gamma}\left(e_{i_{1}}, \ldots, e_{i_{r}}\right), \\
& =\sum_{(\gamma)} T_{\gamma} \varepsilon_{I}^{\gamma} .
\end{aligned}
$$

Since $I$ is arbitrary in the equation above, for each increasing $\gamma_{0}$ we may choose $I=\gamma_{0}$ so that $\varepsilon_{I}^{\gamma_{0}}=1$ and $\varepsilon_{I}^{\gamma}=0$ for $\gamma \neq \gamma_{0}$. It follows that $T_{\gamma_{0}}=0$.

With the representation of alternating tensors as in (4.19) the action of a flux may be represented as

$$
\begin{aligned}
T\left(v_{1}, \ldots, v_{r}\right) & =\sum_{(\gamma)} T_{\gamma} e^{\gamma_{1}} \wedge \cdots \wedge e^{\gamma_{r}}\left(\sum_{i_{1}} v_{1}^{i_{1}} e_{i_{1}}, \ldots, \sum_{i_{r}} v_{r}^{i_{r}} e_{i_{r}}\right), \\
& =\sum_{(\gamma), I} T_{\gamma} v_{1}^{i_{1}} \cdots v_{r}^{i_{r}} e^{\gamma_{1}} \wedge \cdots \wedge e^{\gamma_{r}}\left(e_{i_{1}}, \ldots, e_{i_{r}}\right),
\end{aligned}
$$

and so, as expected,

$$
T\left(v_{1}, \ldots, v_{r}\right)=\sum_{(\gamma), I} T_{\gamma} v_{1}^{i_{1}} \cdots v_{r}^{i_{r}} \varepsilon_{I}^{\gamma}=\sum_{I} T_{I} v_{1}^{i_{1}} \cdots v_{r}^{i_{r}} .
$$

4.5. Simple Multivectors. Writing, $e_{\beta}=e_{\beta_{1}} \wedge \cdots \wedge e_{\beta_{r}}$ for an increasing multiindex $\beta$, one has by (4.I) and (4.20)

$$
e^{\gamma}\left(e_{\beta}\right)=\varepsilon_{\beta}^{\gamma} .
$$

Since both multi-indices are increasing, this expression equals either I or $\mathrm{O}$. 
A multivector of the form $v=v_{1} \wedge \cdots \wedge v_{r}$ will be referred to as a simple multivector. Similarly, an alternating multilinear map of the form $T=\phi^{1} \wedge \cdots \wedge \phi^{r}$, for a collection of linearly independent covectors $\phi_{1}, \ldots, \phi_{r}$, will be referred to as a simple multicovector. By the linearity property of the flux operation, $(4.2,4.8)$, it follows that for a simple multivector

$$
\begin{aligned}
v & =v_{1} \wedge \cdots \wedge v_{r}, \\
& =\left(\sum_{i_{1}} v_{1}^{i_{1}} e_{i_{1}}\right) \wedge \cdots \wedge\left(\sum_{i_{r}} v_{r}^{i_{r}} e_{i_{r}}\right), \\
& =\sum_{i_{1}, \ldots, i_{r}} v_{1}^{i_{1}} \cdots v_{r}^{i_{r}} e_{i_{1}} \wedge \cdots \wedge e_{i_{r}} .
\end{aligned}
$$

Adding up simple multivectors to obtain a general multivector will not alter the form of this representation so that any multivector $v$ may be represented in the form

$$
v=\sum_{i_{1}, \ldots, i_{r}} v^{i_{1} \ldots i_{r}} e_{i_{1}} \wedge \cdots \wedge e_{i_{r}} .
$$

Using Equation (3.I2), $e_{i_{1}} \wedge \cdots \wedge e_{i_{r}}=0$ if two or more indices are equal and it follows that the values for the multi-index $I$ for non-vanishing terms may be written as a composition $I=\gamma \circ \pi$ where $\pi$ is a permutation of $r$ symbols and $\gamma:\{1, \ldots, r\} \rightarrow\{1, \ldots, n\}$ is increasing. Thus, for a nonvanishing term

$$
e_{i_{1}} \wedge \cdots \wedge e_{i_{r}}=(-1)^{|\pi|} e_{\gamma_{1}} \wedge \cdots \wedge e_{\gamma_{r}},
$$

and

$$
v=\sum_{\pi, \gamma}(-1)^{|\pi|} v^{(\gamma \circ \pi)_{1} \ldots(\gamma \circ \pi)_{r}} e_{\gamma_{1}} \wedge \cdots \wedge e_{\gamma_{r}}
$$

Set

$$
v^{\gamma_{1} \ldots \gamma_{r}}=\sum_{\pi}(-1)^{|\pi|} v^{(\gamma \circ \pi)_{1} \ldots(\gamma \circ \pi)_{r}}=\sum_{\pi} \varepsilon_{\pi_{1} \ldots \pi_{r}} v^{(\gamma \circ \pi)_{1} \ldots(\gamma \circ \pi)_{r},}
$$

then, a multivector may be represented in the form

$$
v=\sum_{(\gamma)} v^{\gamma} e_{\gamma}, \quad \gamma_{i}=1, \ldots, n .
$$

In particular, it follows from (4.28) that

$$
e^{\gamma}(v)=v^{\gamma}
$$

The multivectors $\left\{e_{\gamma}\right\}$, for an increasing $\gamma$, are linearly independent and it follows that they form a basis for the space of multivectors.

4.6. Alternation. Alternation is the action of extracting an alternating $r$-tensor from an arbitrary $r$-tensor $T$. It is desirable that the alternation action will be a projection, i.e., when applied to an alternating tensor, it will not change it. The alternation operator is defined by

$$
\mathfrak{A}(T)\left(v_{1}, \ldots, v_{r}\right)=\frac{1}{r !} \sum_{\pi}(-1)^{|\pi|} T\left(v_{\pi_{1}}, \ldots, v_{\pi_{r}}\right) .
$$


To show that $\mathfrak{A}(T)$ is indeed alternating, we observe that (4.36) implies that for a permutation $\rho$,

$$
\begin{aligned}
\mathfrak{A}(T)\left(v_{\rho_{1}}, \ldots, v_{\rho_{r}}\right) & =\frac{1}{r !} \sum_{\pi}(-1)^{|\pi|} T\left(v_{(\pi \circ \rho)_{1}}, \ldots, v_{(\pi \circ \rho)_{r}}\right), \\
& =\frac{1}{r !} \sum_{\mu}(-1)^{|\mu|-|\rho|} T\left(v_{\mu_{1}}, \ldots, v_{\mu_{r}}\right), \\
& =\frac{1}{r !}(-1)^{|\rho|} \sum_{\mu}(-1)^{|\mu|} T\left(v_{\mu_{1}}, \ldots, v_{\mu_{r}}\right), \\
& =(-1)^{|\rho|} \mathfrak{A}(T)\left(v_{1}, \ldots, v_{r}\right),
\end{aligned}
$$

where in the second line we used $\mu=\pi \circ \rho,|\mu|=|\pi|+|\rho|$.

In case $T$ is alternating

$$
\begin{aligned}
\mathfrak{A}(T)\left(v_{1}, \ldots, v_{r}\right) & =\frac{1}{r !} \sum_{\pi}(-1)^{|\pi|} T\left(v_{\pi_{1}}, \ldots, v_{\pi_{r}}\right), \\
& =\frac{1}{r !} \sum_{\pi}(-1)^{|\pi|}(-1)^{|\pi|} T\left(v_{1}, \ldots, v_{r}\right), \\
& =T\left(v_{1}, \ldots, v_{r}\right),
\end{aligned}
$$

so $\mathfrak{A}$ leaves alternating tensors invariant.

For an $r$-tensor $T=\sum_{I} T_{i_{1} \ldots i_{r}} e^{i_{1}} \otimes \cdots \otimes e^{i_{r}}$, one has

$$
\begin{aligned}
\mathfrak{A}(T)\left(v_{1}, \ldots, v_{r}\right) & =\frac{1}{r !} \sum_{\pi, I}(-1)^{|\pi|} T_{i_{1} \ldots i_{r}} e^{i_{1}} \otimes \cdots \otimes e^{i_{r}}\left(v_{\pi_{1}}, \ldots, v_{\pi_{r}}\right), \\
& =\frac{1}{r !} \sum_{\pi, I}(-1)^{|\pi|} T_{i_{1} \ldots i_{r}} e^{i_{1}}\left(v_{\pi_{1}}\right) \cdots e^{i_{r}}\left(v_{\pi_{r}}\right), \\
& =\frac{1}{r !} \sum_{\pi, I}(-1)^{|\pi|} T_{i_{1} \ldots i_{r}} e^{\left(I \circ \pi^{-1}\right)_{1}}\left(v_{1}\right) \cdots e^{\left(I \circ \pi^{-1}\right)_{r}}\left(v_{r}\right), \\
& =\frac{1}{r !} \sum_{\pi, I}(-1)^{|\pi|} T_{i_{1} \ldots i_{r}} e^{\left(I \circ \pi^{-1}\right)_{1}} \otimes \cdots \otimes e^{\left(I \circ \pi^{-1}\right)_{r}}\left(v_{1}, \ldots, v_{r}\right),
\end{aligned}
$$

from which

$$
\mathfrak{A}(T)=\frac{1}{r !} \sum_{\pi, I}(-1)^{|\pi|} T_{i_{1} \ldots i_{r}} e^{\left(I \circ \pi^{-1}\right)_{1}} \otimes \cdots \otimes e^{\left(I \circ \pi^{-1}\right)_{r}} .
$$

In particular, setting $T_{i_{1} \ldots i_{r}}=\delta_{i_{1}}^{j_{1}} \cdots \delta_{i_{r}}^{j_{r}}$, for some multi-index $J$,

$$
\begin{aligned}
\mathfrak{A}\left(e^{j_{1}} \otimes \cdots \otimes e^{j_{r}}\right) & =\frac{1}{r !} \sum_{\pi}(-1)^{|\pi|} e^{\left(J \circ \pi^{-1}\right)_{1}} \otimes \cdots \otimes e^{\left(J \circ \pi^{-1}\right)_{r},} \\
& =\frac{1}{r !} \sum_{I} \varepsilon_{i_{1} \ldots i_{r}}^{j_{1} \ldots j_{r}} e^{i_{1}} \otimes \cdots \otimes e^{i_{r}},
\end{aligned}
$$


where in the last equality we set $I=J \circ \pi^{-1}$ and used $|I|=|J|+|\pi|,(-1)^{|\pi|}=$ $(-1)^{|I|-|J|}=\varepsilon_{i_{1} \ldots i_{r}}^{j_{1} \ldots j_{r}}$ for each nonvanishing term. It follows that $\mathfrak{A}\left(e^{j_{1}} \otimes \cdots \otimes e^{j_{r}}\right)=$ 0 if two or more of the indices are identical. In addition, using the definitions of $e^{I}=e^{i_{1}} \wedge \cdots \wedge e^{i_{r}}$ in (4.I9), we record that

$$
e^{I}=e^{i_{1}} \wedge \cdots \wedge e^{i_{r}}=r ! \mathfrak{A}\left(e^{j_{1}} \otimes \cdots \otimes e^{j_{r}}\right)=\sum_{I} \varepsilon_{i_{1} \ldots i_{r}}^{j_{1} \ldots j_{r}} e^{i_{1}} \otimes \cdots \otimes e^{i_{r}}
$$

The previous equations are not limited to elements of the dual basis. If $\phi^{1}, \ldots, \phi^{r} \in$ $\mathbf{V}^{*}$ are $r$ covectors, then, for $j_{1}, \ldots, j_{r} \in\{1, \ldots, r\}$

$$
\begin{aligned}
\mathfrak{A}\left(\phi^{j_{1}} \otimes \cdots \otimes \phi^{j_{r}}\right) & =\frac{1}{r !} \sum_{\pi}(-1)^{|\pi|} \phi^{\left(J \circ \pi^{-1}\right)_{1}} \otimes \cdots \otimes \phi^{\left(J \circ \pi^{-1}\right)_{r}}, \\
& =\frac{1}{r !} \sum_{I} \varepsilon_{i_{1} \ldots i_{r}}^{j_{1} \ldots j_{r}} \phi^{i_{1}} \otimes \cdots \otimes \phi^{i_{r}}
\end{aligned}
$$

The last equation implies that for any permutation $\pi$,

$$
\mathfrak{A}\left(\phi^{\pi_{1}} \otimes \cdots \otimes \phi^{\pi_{r}}\right)=(-1)^{|\pi|} \mathfrak{A}\left(\phi^{1} \otimes \cdots \otimes \phi^{r}\right) .
$$

It is observed that by $(4.40)$ the alternation operator is linear and in particular,

$$
\mathfrak{A}(T)=\sum_{I} T_{i_{1} \ldots i_{r}} \mathfrak{A}\left(e^{i_{1}} \otimes \cdots \otimes e^{i_{r}}\right) .
$$

We conclude that $\mathfrak{A}$ is indeed a linear projection of the vector space of $r$-tensors on the space of $r$-alternating tensors

4.7. Exterior Products. The notation $e^{\gamma_{1}} \wedge \cdots \wedge e^{\gamma_{r}}$ was introduced formally by $(4.19,4.2 \mathrm{I})$ without an explanation or motivation. In this section we present the exterior product of alternating tensors, denoted by $\wedge$ for which the expression above is a special case.

We observe first that Equations (4.I9,4.2I) need not be restricted only to elements of the dual basis. In fact, if $\phi^{1}, \ldots, \phi^{r} \in \mathbf{V}^{*}$ are $r$ covectors, one may set

$$
\phi^{1} \wedge \cdots \wedge \phi^{r}=r ! \mathfrak{A}\left(\phi^{1} \otimes \cdots \otimes \phi^{r}\right)=\sum_{\pi}(-1)^{|\pi|} \phi^{\pi_{1}} \otimes \cdots \otimes \phi^{\pi_{r}} .
$$

From Equation (4.44), it follows that

$$
\begin{aligned}
\phi^{\pi_{1}} \wedge \cdots \wedge \phi^{\pi_{r}} & =(-1)^{|\pi|} \phi^{1} \wedge \cdots \wedge \phi^{r}, \\
\phi^{(\pi \circ \rho)_{1}} \wedge \cdots \wedge \phi^{(\pi \circ \rho)_{r}} & =(-1)^{|\pi|} \phi^{\rho_{1}} \wedge \cdots \wedge \phi^{\rho_{r}} .
\end{aligned}
$$


For a collection of $r$ linearly independent vectors $v_{1}, \ldots, v_{r}$ one has

$$
\begin{aligned}
\phi^{1} \wedge \cdots \wedge \phi^{r}\left(v_{1}, \ldots, v_{r}\right) & =\sum_{\pi}(-1)^{|\pi|} \phi^{\pi_{1}} \otimes \cdots \otimes \phi^{\pi_{r}}\left(v_{1}, \ldots, v_{r}\right), \\
& =\sum_{\pi}(-1)^{|\pi|} \phi^{\pi_{1}}\left(v_{1}\right) \cdots \phi^{\pi_{r}}\left(v_{r}\right), \\
& =\frac{1}{r !} \sum_{I, J} \varepsilon_{J}^{I} \phi^{j_{1}}\left(v_{i_{1}}\right) \cdots \phi^{j_{r}}\left(v_{i_{r}}\right), \\
& =\operatorname{det}\left[\phi^{i}\left(v_{j}\right)\right] .
\end{aligned}
$$

In particular, the tensors $e^{I}$ satisfy

$$
e^{i_{1}} \wedge \cdots \wedge e^{i_{r}}\left(v_{1}, \ldots, v_{r}\right)=\operatorname{det}\left[\left(v_{j}\right)^{i_{k}}\right], \quad k=1, \ldots, r .
$$

The action of the alternation operator may be represented now using (4.45) and $(4.42)$ as

$$
\mathfrak{A}(T)=\frac{1}{r !} \sum_{I} T_{I} e^{i_{1}} \wedge \cdots \wedge e^{i_{r}}=\frac{1}{r !} \sum_{I} T_{I} e^{I}=\sum_{(\gamma)} \mathfrak{A}(T)_{\gamma} e^{\gamma},
$$

where,

$$
\begin{aligned}
\mathfrak{A}(T)_{\gamma} & =\mathfrak{A}(T)\left(e_{\gamma_{1}}, \ldots, e_{\gamma_{r}}\right), \\
& =\frac{1}{r !} \sum_{I} T_{I} e^{I}\left(e_{\gamma_{1}}, \ldots, e_{\gamma_{r}}\right), \\
& =\frac{1}{r !} \sum_{I} T_{I} \varepsilon_{\gamma}^{I} .
\end{aligned}
$$

For the covectors $\phi^{1}, \ldots, \phi^{r+p}$, let $T=\phi^{1} \wedge \cdots \wedge \phi^{r}$ and $S=\phi^{r+1} \wedge \cdots \wedge \phi^{r+p}$ We set (see [Whis7, p. 4I])

$$
T \wedge S=\phi^{1} \wedge \cdots \wedge \phi^{r} \wedge \phi^{r+1} \wedge \cdots \wedge \phi^{r+p}
$$

and consider $\mathfrak{A}(T \otimes S)$. One has,

$$
\begin{aligned}
T \otimes S & =\left(\sum_{\beta}(-1)^{|\beta|} \phi^{\beta_{1}} \otimes \cdots \otimes \phi^{\beta_{r}}\right) \otimes\left(\sum_{\gamma}(-1)^{|\gamma|} \phi^{\gamma_{r+1}} \otimes \cdots \otimes \phi^{\gamma_{r+p}}\right), \\
& =\sum_{\beta, \gamma}(-1)^{|\beta|}(-1)^{|\gamma|} \phi^{\beta_{1}} \otimes \cdots \otimes \phi^{\beta_{r}} \otimes \phi^{\gamma_{r+1}} \otimes \cdots \otimes \phi^{\gamma_{r+p}},
\end{aligned}
$$


where $\beta$ is a permutation of $\{1, \ldots, r\}$ and $\gamma$ is a permutation of $\{r+1, \ldots, r+p\}$.

Thus, letting $\pi$ denote a permutation of $\{1, \ldots, r+p\}$, (4.43) yields

$$
\begin{aligned}
& (r+p) ! \mathfrak{A}(T \otimes S) \\
= & \sum_{\pi, \beta, \gamma}(-1)^{|\pi|}(-1)^{|\beta|}(-1)^{|\gamma|} \phi^{(\pi \circ \beta)_{1}} \otimes \cdots \otimes \phi^{(\pi \circ \beta)_{r}} \otimes \phi^{(\pi \circ \gamma)_{r+1}} \otimes \cdots \otimes \phi^{(\pi \circ \gamma)_{r+p},} \\
= & \sum_{\beta, \gamma}(-1)^{|\beta|}(-1)^{|\gamma|}\left(\sum_{\pi}(-1)^{|\pi|} \phi^{(\pi \circ \beta)_{1}} \otimes \cdots \otimes \phi^{(\pi \circ \beta)_{r}} \otimes \phi^{(\pi \circ \gamma)_{r+1}} \otimes \cdots \otimes \phi^{(\pi \circ \gamma)_{r+p}}\right), \\
= & \sum_{\beta, \gamma}(-1)^{|\beta|}(-1)^{|\gamma|} \phi^{\beta_{1}} \wedge \cdots \wedge \phi^{\beta_{r}} \wedge \phi^{\gamma_{r+1}} \wedge \cdots \wedge \phi^{\gamma_{r+p}}, \\
= & \sum_{\beta, \gamma}(-1)^{|\beta|}(-1)^{|\gamma|}(-1)^{|\beta|}(-1)^{|\gamma|} \phi^{1} \wedge \cdots \wedge \phi^{r} \wedge \phi^{r+1} \wedge \cdots \wedge \phi^{r+p}, \\
= & r ! p ! \phi^{1} \wedge \cdots \wedge \phi^{r} \wedge \phi^{r+1} \wedge \cdots \wedge \phi^{r+p},
\end{aligned}
$$

where we used (4.47) in the fifth line. We conclude that

$$
T \wedge S=\frac{(r+p) !}{r ! p !} \mathfrak{A}(T \otimes S) .
$$

The exterior product of simple alternating tensors, as defined above, may be extended to exterior product of any two tensors, particularly, two alternating tensors. For any two tensors $T$ and $S$, one may define the exterior product $T \wedge S$ by using Equation (4.55). Since the alternation operator is linear, it follows that this definition is compatible with the definition of the exterior product of two simple alternating tensors. and with the representations of alternating tensors in terms of the simple multi-covectors $\left\{e^{\gamma_{1}} \wedge \cdots \wedge e^{\gamma_{r}}\right\}$. Specifically, for an $r$-alternating tensor $T$ and a $p$-alternating tensor $S$, one has

$$
\begin{aligned}
T \wedge S & =\frac{(r+p) !}{r ! p !} \mathfrak{A}(T \otimes S) \\
& =\frac{(r+p) !}{r ! p !} \mathfrak{A}\left[\left(\frac{1}{r !} \sum_{I} T_{I} e^{i_{1}} \wedge \cdots \wedge e^{i_{r}}\right) \otimes\left(\frac{1}{p !} \sum_{J} S_{J} e^{j_{r+1}} \wedge \cdots \wedge e^{j_{r+p}}\right)\right], \\
& =\frac{(r+p) !}{r ! p !} \sum_{I, J} \frac{1}{r ! p !} T_{I} S_{J} \mathfrak{A}\left[\left(e^{i_{1}} \wedge \cdots \wedge e^{i_{r}}\right) \otimes\left(e^{j_{r+1}} \wedge \cdots \wedge e^{j_{r+p}}\right)\right], \\
& =\frac{(r+p) !}{r ! p !} \sum_{I, J} \frac{1}{(r+p) !} T_{I} S_{J} e^{i_{1}} \wedge \cdots \wedge e^{i_{r}} \wedge e^{j_{r+1}} \wedge \cdots \wedge e^{j_{r+p}}, \\
& =\frac{1}{r ! p !} \sum_{I, J} T_{I} S_{J} e^{i_{1}} \wedge \cdots \wedge e^{i_{r}} \wedge e^{j_{r+1}} \wedge \cdots \wedge e^{j_{r+p}},
\end{aligned}
$$

where $I:\{1, \ldots, r\} \rightarrow\{1, \ldots, n\}, J:\{r+1, \ldots, r+p\} \rightarrow\{1, \ldots, n\}$. (It is noted that one could write $j_{1}, \ldots, j_{p}$ for $j_{r+1}, \ldots, j_{r+p}$ so that $J:\{1, \ldots, p\} \rightarrow$ 
$\{1, \ldots, n\}$.) Each nonvanishing term above may be represented by a multi-index $K:\{1, \ldots, r+p\} \rightarrow\{1, \ldots, n\}$ such that $\left.K\right|_{\{1, \ldots, r\}}=I,\left.K\right|_{\{r+1, \ldots, r+p\}}=J$, and

$$
T \wedge S=\frac{1}{r ! p !} \sum_{K} T_{k_{1} \ldots k_{r}} S_{k_{r+1} \ldots k_{r+p}} e^{k_{1}} \wedge \cdots \wedge e^{k_{r+p}}
$$

Returning to the last line of (4.56), we may write $I=\alpha \circ \pi^{\prime}, J=\beta \circ \pi$ ", where $\alpha$ and $\beta$ are increasing and $\pi^{\prime}, \pi^{\prime \prime}$ are permutations of $\{1, \ldots, r\}$ and $\{r+1, \ldots, r+p\}$, respectively. The decomposition is obviously unique. Thus,

$$
\begin{aligned}
T \wedge S & =\frac{1}{r ! p !} \sum_{(\alpha),(\beta)}\left(\sum_{\pi^{\prime}, \pi^{\prime}} T_{\alpha \circ \pi^{\prime}} S_{\beta \circ \pi^{\prime \prime}} e^{\left(\alpha \circ \pi^{\prime}\right)_{1}} \wedge \cdots \wedge e^{\left(\alpha \circ \pi^{\prime}\right)_{r}} \wedge e^{\left(\beta \circ \pi^{\prime \prime}\right)_{r+1}} \wedge \cdots \wedge e^{\left(\beta \circ \pi^{\prime \prime}\right)_{r+p}}\right) \\
& =\frac{1}{r ! p !} \sum_{(\alpha),(\beta)}\left(\sum_{\pi^{\prime}, \pi^{\prime}}(-1)^{2\left(\left|\pi^{\prime}\right|+\left|\pi^{\prime \prime}\right|\right)} T_{\alpha} S_{\beta} e^{\alpha_{1}} \wedge \cdots \wedge e^{\alpha_{r}} \wedge e^{\beta_{r+1}} \wedge \cdots \wedge e^{\beta_{r+p}}\right) \\
& =\sum_{(\alpha),(\beta)} T_{\alpha} S_{\beta} e^{\alpha_{1}} \wedge \cdots \wedge e^{\alpha_{r}} \wedge e^{\beta_{r+1}} \wedge \cdots \wedge e^{\beta_{r+p}} \\
& =\sum_{\mu} T_{\mu_{1} \ldots \mu_{r}} S_{\mu_{r+1} \ldots \mu_{r+p}} e^{\mu_{1}} \wedge \cdots \wedge e^{\mu_{r+p}}
\end{aligned}
$$

where the multi-indices $\mu$ are injections $\{1, \ldots, r+p\} \rightarrow\{1, \ldots, n\}$ such that the restrictions $\left.\mu\right|_{\{1, \ldots, r\}}$ and $\left.\mu\right|_{\{r+1, \ldots, r+p\}}$ are increasing. Each such multi-index $\mu$ may be represented uniquely in the form $\mu=\gamma \circ \rho$, where $\gamma$ is increasing and $\rho$ is a permutation of $\{1, \ldots, r+p\}$ such that $\left.\rho\right|_{\{1, \ldots, r\}}$ and $\left.\rho\right|_{\{r+1, \ldots, r+p\}}$ are increasing. (See [dR84, p. I8] and [Whi57, p. 4I].) A permutation such as $\rho$ is referred to as an $(r, p)$-shuffle (e.g., [AMR88, p. 394]). Using this representation in (4.58) we have

$$
\begin{aligned}
T \wedge S & =\sum_{(\gamma)}\left(\sum_{\rho} T_{(\gamma \circ \rho)_{1} \ldots(\gamma \circ \rho)_{r}} S_{(\gamma \circ \rho)_{r+1} \ldots(\gamma \circ \rho)_{r+p}} e^{(\gamma \circ \rho)_{1}} \wedge \cdots \wedge e^{(\gamma \circ \rho)_{r+p}}\right), \\
& =\sum_{(\gamma)}\left(\sum_{\rho}(-1)^{|\rho|} T_{(\gamma \circ \rho)_{1} \ldots(\gamma \circ \rho)_{r}} S_{(\gamma \circ \rho)_{r+1} \ldots(\gamma \circ \rho)_{r+p}} e^{\gamma_{1}} \wedge \cdots \wedge e^{\gamma_{r+p}}\right),
\end{aligned}
$$

and so

$$
\begin{aligned}
(T \wedge S)_{\gamma} & =\sum_{\rho}(-1)^{|\rho|} T_{(\gamma \circ \rho)_{1} \ldots(\gamma \circ \rho)_{r}} S_{(\gamma \circ \rho)_{r+1} \ldots(\gamma \circ \rho)_{r+p}}, \\
& =\sum_{\mu} \varepsilon_{\gamma_{1} \ldots, \gamma_{r+p}}^{\mu_{1} \ldots \mu_{r+p}} T_{\mu_{1} \ldots \mu_{r}} S_{\mu_{r+1} \ldots \mu_{r+p}} .
\end{aligned}
$$

where the sums are taken over shuffles $\rho$ and $\mu=\gamma \circ \rho$. Clearly, one could obtain the last equation by starting with the representations of $T$ and $S$ in terms of the multivectors $e^{\gamma_{1}} \wedge \cdots \wedge e^{\gamma_{r}}$ and $e^{\gamma_{r+1}} \wedge \cdots \wedge e^{\gamma_{r+p}}$ as in Equation (4.I9). 
Using Equations (4.36), (4.55) and (4.56), the action of the exterior product may be written as

$$
T \wedge S\left(v_{1}, \ldots, v_{r+p}\right)=\frac{1}{r ! p !} \sum_{\pi}(-1)^{|\pi|} T\left(v_{\pi_{1}}, \ldots, v_{\pi_{r}}\right) S\left(v_{\pi_{r+1}}, \ldots, v_{\pi_{r+p}}\right)
$$

Here, $\pi$ is not a shuffle so that for example $\pi_{1}, \ldots, \pi_{r}$ need not be increasing. However, we can write each such permutation as follows. For $i=1, \ldots, r$, set $\pi_{i}=(\alpha \circ \mu)_{i}$, where $\mu$ is an $(r, p)$-shuffle and $\alpha$ is a permutation of $\{1, \ldots, r\}$. For $i=r+1, \ldots, r+p$, set $\pi_{i}=(\beta \circ \mu)_{i}$, where $\beta$ is a permutation of $p$ symbols. Hence, with $|\pi|=|\mu|+|\alpha|+|\beta|$,

$$
\begin{aligned}
& T \wedge S\left(v_{1}, \ldots, v_{r+p}\right) \\
& =\frac{1}{r ! p !} \sum_{\mu, \alpha, \beta}(-1)^{|\mu|+|\alpha|+|\beta|} T\left(v_{(\alpha \circ \mu)_{1}}, \ldots, v_{(\alpha \circ \mu)_{r}}\right) S\left(v_{(\beta \circ \mu)_{r+1}}, \ldots, v_{(\beta \circ \mu)_{r+p}}\right), \\
& =\frac{1}{r ! p !} \sum_{\mu, \alpha, \beta}(-1)^{|\mu|+|\alpha|+|\beta|}(-1)^{|\alpha|}(-1)^{|\beta|} T\left(v_{\mu_{1}}, \ldots, v_{\mu_{r}}\right) S\left(v_{\mu_{r+1}}, \ldots, v_{\mu_{r+p}}\right), \\
& =\frac{1}{r ! p !} \sum_{\mu, \alpha, \beta}(-1)^{|\mu|} T\left(v_{\mu_{1}}, \ldots, v_{\mu_{r}}\right) S\left(v_{\mu_{r+1}}, \ldots, v_{\mu_{r+p}}\right),
\end{aligned}
$$

so taking account of the number of permutations $\alpha$ and $\beta$ we have

$$
T \wedge S\left(v_{1}, \ldots, v_{r+p}\right)=\sum_{\mu}(-1)^{|\mu|} T\left(v_{\mu_{1}}, \ldots, v_{\mu_{r}}\right) S\left(v_{\mu_{r+1}}, \ldots, v_{\mu_{r+p}}\right),
$$

where the sum is taken over all $(r, p)$-shuffles $\mu$.

Remark 4.I. There are other conventions for the definition of the exterior product. See for example [War83, p. 60], [Whis7] and [AMR88]. From the point of view of fluxes as presented here, one may think of the other conventions as pertaining to the interpretation of $v_{1} \wedge \cdots \wedge v_{r}$ as the extent of the parallelepiped generated by the vectors rather than the extent of the simplex.

Example 4.2. Consider the case where $T$ is a I-alternating tensor, i.e., an element of the dual space $\mathbf{V}^{*}$. Thus, if we have the component representations $T=\sum_{i} T_{i} e^{i}$, $S=\sum_{(\beta)} S_{\beta} e^{\beta_{1}} \wedge \cdots \wedge e^{\beta_{p}}$, Equation (4.52) and linearity imply that

$$
T \wedge S=\sum_{(\beta), i} T_{i} S_{\beta} e^{i} \wedge e^{\beta_{1}} \wedge \cdots \wedge e^{\beta_{p}} .
$$

Alternatively, we may use Equation (4.60) noting that for the case $r=1$ considered, any $(1, p)$ shuffle $\rho$ is of the form $(1, \ldots, i, \ldots, p+1) \mapsto(i, 1, \ldots, \widehat{i}, \ldots, p+1)$ so that $|\rho|=i-1$. It follows that

$$
(T \wedge S)_{\gamma}=\sum_{i}(-1)^{i-1} T_{\gamma_{i}} S_{\gamma_{1} \ldots \gamma_{i} \ldots \gamma_{p+1}},
$$


and

$$
\begin{aligned}
T \wedge S & =\sum_{(\gamma), i}(-1)^{i-1} T_{\gamma_{i}} S_{\gamma_{1} \ldots \gamma_{i} \ldots \gamma_{p+1}} e^{\gamma_{1}} \wedge \cdots \wedge e^{\gamma_{p+1}}, \\
& =\sum_{(\gamma), i} T_{\gamma_{i}} S_{\gamma_{1} \ldots \widehat{\gamma}_{i} \ldots \gamma_{p+1}} e^{\gamma_{i}} \wedge e^{\gamma_{1}} \wedge \cdots \wedge \widehat{e^{\gamma_{i}}} \wedge \cdots \wedge e^{\gamma_{p+1}} .
\end{aligned}
$$

Example 4.3. Consider the relation between $S \wedge T$ and $T \wedge S$ for an $r$-alternating tensor $T$ and a $p$-alternating tensor $S$. From Equation (4.56) and counting the number of permutations needed in order to arrive from $e^{i_{1}} \wedge \cdots \wedge e^{i_{r}} \wedge e^{j_{1}} \wedge \cdots \wedge e^{j_{p}}$ to $e^{j_{1}} \wedge \cdots \wedge e^{j_{p}} \wedge e^{i_{1}} \wedge \cdots \wedge e^{i_{r}}$, it follows that

$$
S \wedge T=(-1)^{r p} T \wedge S .
$$

In particular, if either $r$ or $p$ are even, $S \wedge T=T \wedge S$.

4.8. The spaces of Multivectors and Multicovectors. The space of alternating $r$-tensors on the space $\mathbf{V}$ will be denoted by $\bigwedge^{r}\left(\mathbf{V}^{*}\right)$. We have seen how the collection of multicovectors $\left\{e^{\gamma_{1}} \wedge \cdots \wedge e^{\gamma_{r}}\right\}$, for all increasing multi-indices $\gamma$, form a basis for $\bigwedge^{r}\left(\mathbf{V}^{*}\right)$. It follows that the dimension of $\bigwedge^{r}\left(\mathbf{V}^{*}\right)$ is the number of ways one can choose $r$ numbers out of $n$, i.e.,

$$
\operatorname{dim} \bigwedge\left(\mathbf{V}^{*}\right)=\frac{n !}{r !(n-r) !}
$$

In particular, $\operatorname{dim} \bigwedge^{0}\left(\mathbf{V}^{*}\right)=\operatorname{dim} \bigwedge^{n}\left(\mathbf{V}^{*}\right)=1$, and $\operatorname{dim} \bigwedge^{1}\left(\mathbf{V}^{*}\right)=\operatorname{dim} \bigwedge^{n-1}\left(\mathbf{V}^{*}\right)=$ $n$. The basis of $\bigwedge^{0}\left(\mathbf{V}^{*}\right)$ contains no elements of the basis of $\mathbf{V}^{*}$ and so $\bigwedge^{0}\left(\mathbf{V}^{*}\right)$ may be identified with the real numbers. For $r>n$, the vector space $\bigwedge^{r}\left(\mathbf{V}^{*}\right)$ is trivial.

The space of $r$-multivectors in $\mathbf{V}$ will be denoted by $\bigwedge^{r}(\mathbf{V})$. By the representations of multivectors and multicovectors in terms of base vectors as in Section 4.4 and Equation (4.34),

$$
\operatorname{dim} \bigwedge^{r}(\mathbf{V})=\operatorname{dim} \bigwedge^{r}\left(\mathbf{V}^{*}\right)
$$

The alternating multilinear action of a flux $T$ on vectors was represented in (4.7) by a linear action of $T$ on simple multivectors and extended by (4.II) to a linear action on non-simple multivectors. We conclude that

$$
\bigwedge^{r}\left(\mathbf{V}^{*}\right)=\left(\bigwedge^{r}(\mathbf{V})\right)^{*}
$$

Example 4.4. Algebraic Cauchy Formula with Components. Consider the case where $r=n$ and let $\left\{e_{1}, \ldots, e_{n}\right\}$ be a basis for $\mathbf{V}$. We want to compute the total flux through the $(n-1)$-simplex $\tau$ determined by the vectors $v_{1}, \ldots, v_{n-1}$ for a flux $T$ represented as in Equation (3.45) by an alternating tensor $J=\sum_{(\alpha)} J_{\alpha_{1} \ldots \alpha_{n-1}} e^{\alpha_{1}} \wedge$ $\cdots \wedge e^{\alpha_{n-1}}$. Since $\alpha$ is increasing and contains $n-1$ out of the $n$ numbers $\{1, \ldots, n\}$, there is a unique $k \in\{1, \ldots, n\}$ such that $\left(\alpha_{1}, \ldots, \alpha_{n-1}\right)=(1, \ldots, \widehat{k}, \ldots, n\}$. It follows that any $(n-1)$-alternating tensor $J$ is of the form

$$
J=\sum_{k} J_{1 \ldots \widehat{k} \ldots n} e^{1} \wedge \cdots \wedge \widehat{e^{k}} \wedge \cdots \wedge e^{n} .
$$


Thus,

$$
\begin{aligned}
T(\tau) & =J\left(v_{1}, \ldots, v_{n-1}\right), \\
& =\sum_{k} J_{1 \ldots \widehat{k} \ldots n} e^{1} \wedge \cdots \wedge \widehat{e^{k}} \wedge \cdots \wedge e^{n}\left(\sum_{i_{1}} v_{1}^{i_{1}} e_{i_{1}}, \ldots, \sum_{i_{n-1}} v_{n-1}^{i_{n-1}} e_{i_{n-1}}\right), \\
& =\sum_{k, I} J_{1 \ldots \widehat{k} \ldots m} v_{1}^{i_{1}} \cdots v_{n-1}^{i_{n-1}} e^{1} \wedge \cdots \wedge \widehat{e^{k}} \wedge \cdots \wedge e^{n}\left(e_{i_{1}}, \ldots, e_{i_{n-1}}\right), \\
& =\sum_{k, I} J_{1 \ldots \widehat{k} \ldots n} v_{1}^{i_{1}} \cdots v_{n-1}^{i_{n-1}} \varepsilon_{i_{1} \ldots \ldots i_{n-1}}^{1 \ldots \widehat{k} \ldots n} .
\end{aligned}
$$

Since

we have

$$
\varepsilon_{i_{1} \ldots \ldots i_{n-1}}^{1 \ldots \widehat{k} \ldots n}=\varepsilon_{k i_{1} \ldots \ldots i_{n-1}}^{k 1 \ldots \widehat{k} \ldots n}=(-1)^{k-1} \varepsilon_{k i_{1} \ldots i_{n-1}}^{1 \ldots \ldots n},
$$

$$
\begin{aligned}
T(\tau)=J\left(v_{1}, \ldots, v_{n-1}\right) & =\sum_{k, I}\left[(-1)^{k-1} J_{1 \ldots \widehat{k} \ldots n}\right] v_{1}^{i_{1}} \cdots v_{n-1}^{i_{n-1}} \varepsilon_{k i_{1} \ldots i_{n-1}}^{1 \ldots \ldots n}, \\
& =\operatorname{det}\left(\left[(-1)^{k-1} J_{1 \ldots \widehat{k} \ldots n}\right] ; v_{1}^{i_{1}} ; \ldots ; v_{n-1}^{i_{n-1}}\right),
\end{aligned}
$$

where the determinant is evaluated for the column vectors indicated by the indices $k, i_{1}, \ldots, i_{n-1}$.

Consider the expression for the flux in a 3-dimensional Euclidean space using the traditional Cauchy formula (3.50) relative to some orthonormal basis,

$$
T(\tau)=\frac{1}{2} \mathbf{J} \cdot(v \times u)=\frac{1}{2} \operatorname{det}\left[\begin{array}{lll}
\mathbf{J}_{1} & v_{1} & u_{1} \\
\mathbf{J}_{2} & v_{2} & u_{2} \\
\mathbf{J}_{3} & v_{3} & u_{3}
\end{array}\right]=\frac{1}{2} \sum_{i, j, k} \varepsilon^{i j k} \mathbf{J}_{i} v_{j} u_{k} .
$$

Comparing the last equality with Equation (4.74) we see that the two equations are equivalent if we make the identification $\mathbf{J}_{k}=2(-1)^{k-1} J_{1 \ldots . . . . . r}$. (The $\frac{1}{2}$ factor is due to the fact that the vector product evaluates the area of the parallelogram while the alternating tensors compute the fluxes through simplexes. It is also noted that $v \times u$ is the analog of the 2-vector $v \wedge u$.

Example 4.5. Consider the space $L\left(\bigwedge^{r}\left(\mathbf{V}^{*}\right), \bigwedge^{n}\left(\mathbf{V}^{*}\right)\right), r<n, n=\operatorname{dim} \mathbf{V}$, of linear mapping $\bigwedge^{r}\left(\mathbf{V}^{*}\right) \rightarrow \bigwedge^{n}\left(\mathbf{V}^{*}\right)$. Set

$$
\iota_{\wedge}: \bigwedge^{n-r}\left(\mathbf{V}^{*}\right) \longrightarrow L\left(\bigwedge^{r}\left(\mathbf{V}^{*}\right), \bigwedge^{n}\left(\mathbf{V}^{*}\right)\right) \text { by } \quad \iota_{\wedge}(T)(S)=T \wedge S
$$

Clearly, $\iota_{\wedge}(T)$ is linear and $\iota_{\wedge}$ is a well defined linear mapping. We also note that $\iota_{\wedge}$ is injective. For if $\iota_{\wedge}(T)=0$, then $\iota_{\wedge}(T)(S)=T \wedge S=0$, for any $r$-alternating mapping $S$. It follows that $T=0$, or otherwise, using components one could write a form $S$ such that $T \wedge S \neq 0$. Thus, $\operatorname{Kernel}\left(\iota_{\wedge}\right)=\{0\}$.

Since $\wedge^{n} \mathbf{V}^{*}$ is I-dimensional,

$$
\operatorname{dim} L\left(\bigwedge^{r}\left(\mathbf{V}^{*}\right), \bigwedge^{n}\left(\mathbf{V}^{*}\right)\right)=\operatorname{dim} \bigwedge^{r}\left(\mathbf{V}^{*}\right)=\operatorname{dim} \bigwedge^{n-r}\left(\mathbf{V}^{*}\right)
$$


It follows that $\iota_{\wedge}$ is an isomorphism and for any element $f \in L\left(\bigwedge^{r}\left(\mathbf{V}^{*}\right), \bigwedge^{n}\left(\mathbf{V}^{*}\right)\right)$ there is an $(n-r)$-alternating tensor $T=\iota_{\wedge}^{-1}(f)$ such that $f(S)=T \wedge S$, for all $r$-alternating tensors $S$.

4.9. Contraction. The contraction $v\lrcorner T$ of an alternating $r$-tensor $T$ and a vector $v$ is an $(r-1)$-alternating tensor defined by

$$
v\lrcorner T\left(v_{2}, \ldots, v_{r}\right)=T\left(v, v_{2}, \ldots, v_{r}\right) .
$$

Clearly, the contraction operation $v\lrcorner T$ is linear in both $v$ and $T$.

Using (4.27) and (4.49) one has

$$
\begin{aligned}
v\lrcorner T\left(v_{2}, \ldots, v_{r}\right) & =\sum_{(\gamma), I} T_{\gamma} v^{i_{1}} v_{2}^{i_{2}} \cdots v_{r}^{i_{r}} \varepsilon_{I}^{\gamma} \\
& =\sum_{(\gamma), I, J} T_{\gamma} v^{i_{1}} \frac{1}{(r-1) !} \varepsilon_{i_{1} j_{1} \ldots j_{r-1}}^{\gamma_{1} \ldots \gamma_{r}} \varepsilon_{i_{2} \ldots i_{r}}^{j_{1} \ldots j_{r-1}} v_{2}^{i_{2}} \cdots v_{r}^{i_{r}}, \\
& =\sum_{(\gamma), I, J} T_{\gamma} v^{i_{1}} \frac{1}{(r-1) !} \varepsilon_{i_{1} j_{1} \ldots j_{r-1}}^{\gamma_{1} \ldots \gamma_{r}} e^{j_{1}} \wedge \cdots \wedge e^{j_{r-1}}\left(v_{2}, \ldots, v_{r}\right), \\
& =\sum_{(\gamma), i,(\beta)} T_{\gamma} v^{i} \varepsilon_{i \beta_{1} \ldots \beta_{r-1}}^{\gamma_{1} \ldots \gamma_{r}} e^{\beta_{2}} \wedge \cdots \wedge e^{\beta_{r-1}}\left(v_{2}, \ldots, v_{r}\right) .
\end{aligned}
$$

where, $I$ is an $r$-multi-index, $J$ is an $(r-1)$-multi-index and $\beta$ is an $(r-1)$ increasing multi-index. It follows that

$$
v\lrcorner T=\sum_{(\gamma), i,(\beta)} T_{\gamma} v^{i} \varepsilon_{i \beta_{1} \ldots \beta_{r-1}}^{\gamma_{1} \ldots \gamma_{r}} e^{\beta_{2}} \wedge \cdots \wedge e^{\beta_{r-1}},
$$

so that

$$
(v\lrcorner T)_{\beta_{1} \ldots \beta_{r-1}}=\sum_{(\gamma), i} T_{\gamma} v^{i} \varepsilon_{i \beta_{1} \ldots \beta_{r-1}}^{\gamma_{1} \ldots \gamma_{r}} .
$$

It is noted that in (4.8I), for a given value of $\beta$ and any increasing multi-index $\gamma$, there is a unique value $i=\gamma \backslash \beta$ for which the Levi-Civita symbol does not vanish. Thus, the sum over $i$ in that equation is superfluous. Its location in the sequence $\gamma_{1} \ldots, \gamma_{r}$ is $\gamma^{-1}(\gamma \backslash \beta)$ and as $\left(\beta_{1}, \ldots, \beta_{r-1}\right)=\left(\gamma_{1}, \ldots, \widehat{\gamma \backslash \beta}, \ldots, \gamma_{r}\right)$, one has

$$
\begin{aligned}
(v\lrcorner T)_{\beta_{1} \ldots \beta_{r-1}} & =\sum_{(\gamma)} T_{\gamma} v^{i} \varepsilon_{i \beta_{1} \ldots \beta_{r-1}}^{\gamma_{1} \ldots \gamma_{r}}, \\
& =\sum_{(\gamma)} T_{\gamma_{1} \ldots \gamma \backslash \beta \ldots \gamma_{r}} v^{\gamma \backslash \beta} \varepsilon_{\gamma \backslash \beta \beta_{1} \ldots \beta_{r-1}}^{\gamma_{1} \ldots \gamma \backslash \beta \gamma_{r}}, \\
& =\sum_{(\gamma)} T_{\gamma_{1} \ldots \gamma \backslash \beta \ldots \gamma_{r}} v^{\gamma \backslash \beta}(-1)^{\gamma^{-1}(\gamma \backslash \beta)-1} .
\end{aligned}
$$

We conclude that

$$
(v\lrcorner T)_{\beta_{1} \ldots \beta_{r-1}}=\sum_{(\gamma)} T_{\gamma \backslash \beta \gamma_{1} \ldots \gamma \backslash \beta \ldots \gamma_{r}} v^{\gamma \backslash \beta} .
$$


Example 4.6. In the special case where $r=n$, an alternating tensor $T$ is represented by a single component $T_{1 \ldots n}$ as $T=T_{1 \ldots n} e^{1} \wedge \cdots \wedge e^{n}$. As in Example 4.4, any $(n-1)$-alternating tensor $S$ is of the form $S=\sum_{k} S_{1 \ldots . .} \ldots n e^{1} \wedge \cdots \wedge e^{k} \wedge \ldots e^{n}$. For an alternating $n$-tensor $T$ and a vector $v$, we consider $v\lrcorner T$. Using the third line of Equation (4.82) with $\beta=\{1, \ldots, \widehat{k}, \ldots, n\}, \gamma \backslash \beta=k, \gamma^{-1}(\gamma \backslash \beta)=k$,

$\left.(v\lrcorner T)_{1 \ldots \widehat{k} \ldots n}=\sum_{k}(-1)^{k-1} T_{1 \ldots n} v^{k}, \quad v\right\lrcorner T=\sum_{k}(-1)^{k-1} T_{1 \ldots n} v^{k} e^{1} \wedge \cdots \wedge e^{k} \wedge \ldots e^{n}$

We conclude that any nonzero $n$-alternating tensor $T$ induces an isomorphism

$$
\iota_{T}: \bigwedge^{n-1}(\mathbf{V}) \longrightarrow \mathbf{V}
$$

defined by the condition that

$$
\left.\iota_{T}(\omega)\right\lrcorner T=\omega .
$$

Equation (4.84) implies that in terms of components

$$
\iota_{T}(S)^{k}=(-1)^{k-1} \frac{S_{1 \ldots \widehat{k} \ldots n}}{T_{1 \ldots n}}
$$

Example 4.7. Consider Equation (4.6I). One may represent any permutation $\pi$ of $r+p$ symbols in the form $\pi=\rho_{i} \circ \mu$ where $\mu$ is a permutation of $\{1, \ldots, r+p\}$ with $\mu_{1}=1$, and $\rho_{i}$ is the permutation of $r+p$ symbols given by

$$
(1,2, \ldots, i, \ldots, r+p) \longmapsto(2, \ldots, i, 1, i+1, \ldots, r+p) .
$$


Clearly, $|\pi|=\left|\rho_{i}\right|+|\mu|=i-1+|\mu|$ so that Equation (4.6I) may be rewritten as

$$
\begin{aligned}
& T \wedge S\left(v_{1}, \ldots, v_{r+p}\right) \\
&=\frac{1}{r ! p !} \sum_{\mu, \rho_{i}}(-1)^{|\mu|}(-1)^{i-1} T\left(v_{\left(\rho_{i} \circ \mu\right)_{1}}, \ldots, v_{\left(\rho_{i} \circ \mu\right)_{r}}\right) S\left(v_{\left(\rho_{i} \circ \mu\right)_{r+1}}, \ldots, v_{\left(\rho_{i} \circ \mu\right)_{r+p}}\right), \\
&=\frac{1}{r ! p !} \sum_{\mu, \rho_{i}, i \leqslant r}(-1)^{|\mu|}(-1)^{i-1} T\left(v_{\left(\rho_{i} \circ \mu\right)_{1}}, \ldots, v_{\left(\rho_{i} \circ \mu\right)_{r}}\right) S\left(v_{\left(\rho_{i} \circ \mu\right)_{r+1}}, \ldots, v_{\left(\rho_{i} \circ \mu\right)_{r+p}}\right) \\
&+\frac{1}{r ! p !} \sum_{\mu, \rho_{i}, i>r}(-1)^{|\mu|}(-1)^{i-1} T\left(v_{\left(\rho_{i} \circ \mu\right)_{1}}, \ldots, v_{\left(\rho_{i} \circ \mu\right)_{r}}\right) S\left(v_{\left(\rho_{i} \circ \mu\right)_{r+1}}, \ldots, v_{\left(\rho_{i} \circ \mu\right)_{r+p}}\right), \\
&=\frac{1}{r ! p !} \sum_{\mu, \rho_{i}, i \leqslant r}(-1)^{|\mu|}(-1)^{2(i-1)} T\left(v_{1}, v_{\mu_{2}} \ldots, v_{\mu_{r}}\right) S\left(v_{\mu_{r+1}}, \ldots, v_{\mu_{r+p}}\right) \\
&+\frac{1}{r ! p !} \sum_{\mu, \rho_{i}, i>r}(-1)^{|\mu|}(-1)^{i-1} T\left(v_{\mu_{2}}, \ldots, v_{\mu_{r+1}}\right)(-1)^{i-r-1} S\left(v_{1}, v_{\mu_{r+2}}, \ldots, v_{\mu_{r+p}}\right), \\
&\left.=\frac{1}{r ! p !} \sum_{\mu, \rho_{i}, i \leqslant r}(-1)^{|\mu|}\left(v_{1}\right\lrcorner T\right)\left(v_{\mu_{2}} \ldots, v_{\mu_{r}}\right) S\left(v_{\mu_{r+1}}, \ldots, v_{\mu_{r+p}}\right) \\
&\left.+\frac{(-1)^{r}}{r ! p !} \sum_{\mu, \rho_{i}, i>r}(-1)^{|\mu|} T\left(v_{\mu_{2}}, \ldots, v_{\mu_{r+1}}\right)\left(v_{1}\right\lrcorner S\right)\left(v_{\mu_{r+2}}, \ldots, v_{\mu_{r+p}}\right), \\
&\left.=\frac{r}{r ! p !} \sum_{\mu}(-1)^{|\mu|}\left(v_{1}\right\lrcorner T\right)\left(v_{\mu_{2}} \ldots, v_{\mu_{r}}\right) S\left(v_{\mu_{r+1}}, \ldots, v_{\mu_{r+p}}\right) \\
&\left.+\frac{(-1)^{r} p}{r ! p !} \sum_{\mu}(-1)^{|\mu|} T\left(v_{\mu_{2}}, \ldots, v_{\mu_{r+1}}\right)\left(v_{1}\right\lrcorner S\right)\left(v_{\mu_{r+2}}, \ldots, v_{\mu_{r+p}}\right), \\
&=\left.\left.\left(v_{1}\right\lrcorner T\right) \wedge S\left(v_{2}, \ldots, v_{r+p}\right)+(-1)^{r} T \wedge\left(v_{1}\right\lrcorner S\right)\left(v_{2}, \ldots, v_{r+p}\right) .
\end{aligned}
$$

It is concluded therefore that

$$
\left.v\lrcorner(T \wedge S)=(v\lrcorner T) \wedge S+(-1)^{r} T \wedge(v\lrcorner S\right) .
$$

Example 4.8. Consider the expression $\left.e_{i_{p}}\right\lrcorner\left(e^{i_{p}} \wedge e^{i_{1}} \wedge \cdots \wedge \widehat{e^{i_{p}}} \wedge \cdots \wedge e^{i_{r}}\right)$ where the indices $i_{1}, \ldots, i_{r}$ are all distinct so that the exterior product does not vanish. Then, using the last equation

$$
\begin{aligned}
\left.e_{i_{p}}\right\lrcorner\left(e^{i_{p}} \wedge e^{i_{1}} \wedge \cdots \wedge \widehat{e^{\widehat{p_{p}}}} \wedge \cdots \wedge e^{i_{r}}\right) & \left.=e_{i_{p}}\right\lrcorner\left(e^{i_{p}} \wedge\left(e^{i_{1}} \wedge \cdots \wedge \widehat{e^{\widehat{l_{p}}}} \wedge \cdots \wedge e^{i_{r}}\right)\right), \\
& \left.=\left(e_{i_{p}}\right\lrcorner e^{i_{p}}\right) \wedge\left(e^{i_{1}} \wedge \cdots \wedge \widehat{e^{\widehat{p}}} \wedge \cdots \wedge e^{i_{r}}\right) \\
& \left.-e^{i_{p}} \wedge\left(e_{i_{p}}\right\lrcorner\left(e^{i_{1}} \wedge \cdots \wedge \widehat{e^{\boldsymbol{l}_{p}}} \wedge \cdots \wedge e^{i_{r}}\right)\right), \\
& =e^{i_{1}} \wedge \cdots \wedge \widehat{e^{\iota_{p}}} \wedge \cdots \wedge e^{i_{r}},
\end{aligned}
$$

where the term in the third line vanishes because the indices are distinct. Using the skew-symmetry of the exterior product we conclude that

$$
\left.e_{i_{p}}\right\lrcorner\left(e^{i_{1}} \wedge \cdots \wedge e^{i_{p}} \wedge \cdots \wedge e^{i_{r}}\right)=(-1)^{p-1} e^{i_{1}} \wedge \cdots \wedge \widehat{e^{l_{p}}} \wedge \cdots \wedge e^{i_{r}},
$$


and

$$
\left.e_{j}\right\lrcorner\left(e^{i_{1}} \wedge \cdots \wedge e^{i_{r}}\right)=\sum_{p} \delta_{j}^{i_{p}}(-1)^{p-1} e^{i_{1}} \wedge \cdots \wedge \widehat{e^{\iota_{p}}} \wedge \cdots \wedge e^{i_{r}} .
$$

Clearly, there can be no more than one non-vanishing term in the last sum.

The following examples will be used in the last section concerning metric free properties of electromagnetism.

Example 4.9. Consider the contraction $v\lrcorner \omega$ for an $(n-1)$ - form $\omega=\sum_{k} \omega_{1 \ldots \widehat{k} \ldots n} e^{1} \wedge$ $\cdots \wedge e^{k} \wedge \ldots e^{n}$. Writing $v=\sum_{i} v^{i} e_{i}$ one has

$$
\begin{aligned}
v\lrcorner \omega & \left.=\left(\sum_{i} v^{i} e_{i}\right)\right\lrcorner\left(\sum_{k} \omega_{1 \ldots \widehat{k} \ldots n} e^{1} \wedge \ldots \wedge \widehat{e^{k}} \wedge \ldots e^{n}\right), \\
& \left.=\sum_{i, k} v^{i} \omega_{1 \ldots \widehat{k} \ldots n} e_{i}\right\lrcorner\left(e^{1} \wedge \ldots \wedge \widehat{e^{k}} \wedge \ldots e^{n}\right) .
\end{aligned}
$$

Note that for $i<k$,

$$
\left.e_{i}\right\lrcorner\left(e^{1} \wedge \cdots \wedge \widehat{e}^{k} \wedge \ldots e^{n}\right)=(-1)^{i-1} e^{1} \wedge \cdots \wedge \widehat{e^{\imath}} \wedge \cdots \wedge \widehat{e^{k}} \wedge \cdots \wedge e^{n},
$$

and for $i>k$

$$
\left.e_{i}\right\lrcorner\left(e^{1} \wedge \cdots \wedge e^{k} \wedge \ldots e^{n}\right)=(-1)^{i-2} e^{1} \wedge \cdots \wedge \widehat{e^{k}} \wedge \cdots \wedge \widehat{e^{l}} \wedge \cdots \wedge e^{n} .
$$

Thus, one has

$$
\begin{aligned}
& v\lrcorner \omega=\sum_{i<k} v^{i} \omega_{1 \ldots \widehat{k} \ldots n}(-1)^{i-1} e^{1} \wedge \cdots \wedge \widehat{e^{l}} \wedge \cdots \wedge \widehat{e}^{k} \wedge \cdots \wedge e^{n} \\
& -\sum_{i>k} v^{i} \omega_{1 \ldots \widehat{k} \ldots n}(-1)^{i-1} e^{1} \wedge \cdots \wedge \widehat{e^{k}} \wedge \cdots \wedge \widehat{e^{\imath}} \wedge \cdots \wedge e^{n},
\end{aligned}
$$

Switching the names of the indices in the preceding line we arrive at

$$
v\lrcorner \omega=\sum_{i<k}\left((-1)^{i-1} v^{i} \omega_{1 \ldots \widehat{k} \ldots n}-(-1)^{k-1} v^{k} \omega_{1 \ldots} \ldots \hat{\imath} \ldots n\right) e^{1} \wedge \cdots \wedge \widehat{e^{l}} \wedge \cdots \wedge \widehat{e^{k}} \wedge \cdots \wedge e^{n} .
$$

Example 4.10. Using the notation of the preceding example, let $\varphi=\sum_{i<j} \varphi_{i j} e^{i} \wedge$ $e^{i}$ be a 2 -form. Using Equation (4.89) and noting that $\varphi \wedge \omega$ as an $(n+1)$-form in an $n$-dimensional space,

$$
\left.0=v\lrcorner(\varphi \wedge \omega)=(v\lrcorner \varphi) \wedge \omega+(-1)^{2} \varphi \wedge(v\lrcorner \omega\right),
$$

and so

$$
(v\lrcorner \varphi) \wedge \omega=-\varphi \wedge(v\lrcorner \omega) .
$$


We will also demonstrate this equation by components computation. We have computed the components of $v\lrcorner \omega$ above and so we may write

$$
\begin{aligned}
& \varphi \wedge(v\lrcorner \omega)=\left(\sum_{i<j} \varphi_{i j} e^{i} \wedge e^{j}\right) \wedge\left[\sum_{p<q}\left((-1)^{p-1} v^{p} \omega_{1 \ldots \widehat{q} \ldots n}-(-1)^{q-1} v^{q} \omega_{1 \ldots \widehat{p} \ldots n}\right)\right. \\
& \left.\left.\cdot e^{1} \wedge \cdots \wedge \widehat{e^{p}} \wedge \cdots \wedge \widehat{e^{q}} \wedge \cdots \wedge e^{n}\right)\right], \\
& =\sum_{i<j, p<q} \varphi_{i j}\left((-1)^{p-1} v^{p} \omega_{1 \ldots \widehat{q} \ldots n}-(-1)^{q-1} v^{q} \omega_{1 \ldots \widehat{p} \ldots n}\right) \\
& \cdot\left(e^{i} \wedge e^{j}\right) \wedge e^{1} \wedge \cdots \wedge \widehat{e^{p}} \wedge \cdots \wedge \widehat{e^{q}} \wedge \cdots \wedge e^{n}, \\
& =\sum_{i<j, p<q} \varphi_{i j}\left((-1)^{p-1} v^{p} \omega_{1 \ldots . . q \ldots n}-(-1)^{q-1} v^{q} \omega_{1 \ldots \widehat{p} \ldots n}\right) \\
& \cdot(-1)^{j-2} \delta^{j q}(-1)^{i-1} \delta^{i p} e^{1} \wedge \cdots \wedge e^{n}, \\
& =-\sum_{i<j} \varphi_{i j}(-1)^{j-1} v^{i} \omega_{1} \ldots \hat{\jmath} \ldots n e^{1} \wedge \cdots \wedge e^{n} \\
& +\sum_{i<j} \varphi_{i j}(-1)^{i-1} v^{j} \omega_{1 \ldots \hat{\imath} \ldots n} e^{1} \wedge \cdots \wedge e^{n} .
\end{aligned}
$$

Now, switching the names of the indices in the first sum of the last equality above one has

$$
\begin{aligned}
\sum_{i<j} \varphi_{i j}(-1)^{j-1} v^{i} \omega_{1 \ldots \hat{\jmath} \ldots n} & =\sum_{j<i} \varphi_{j i}(-1)^{i-1} v^{j} \omega_{1 \ldots \hat{\imath} \ldots n}, \\
& =-\sum_{j<i} \varphi_{i j}(-1)^{i-1} v^{j} \omega_{1 \ldots \hat{\imath} \ldots n},
\end{aligned}
$$

where for $j<i$ we set $\varphi_{i j}=-\varphi_{j i}$. (Recall that $\varphi_{i j}$ was defined originally only for $i<j$.) It follows that

$$
\varphi \wedge(v\lrcorner \omega)=\left(\sum_{j<i} \varphi_{i j}(-1)^{i-1} v^{j} \omega_{1 \ldots \widehat{c} \ldots n}+\sum_{i<j} \varphi_{i j}(-1)^{i-1} v^{j} \omega_{1 \ldots \hat{\imath} \ldots n}\right) e^{1} \wedge \cdots \wedge e^{n}
$$

and we conclude that

$$
\varphi \wedge(v\lrcorner \omega)=\left(\sum_{i, j} \varphi_{i j}(-1)^{i-1} v^{j} \omega_{1} \ldots \hat{\imath} \ldots n\right) e^{1} \wedge \cdots \wedge e^{n}
$$


We now compute the components of $(v\lrcorner \varphi) \wedge \omega$. Firstly,

$$
\begin{aligned}
v\lrcorner \varphi & \left.=\left(\sum_{i} v^{i} e_{i}\right)\right\lrcorner\left(\sum_{p<q} \varphi_{p q} e^{p} \wedge e^{q}\right), \\
& \left.=\sum_{i, p<q} \varphi_{p q} v^{i} e_{i}\right\lrcorner\left(e^{p} \wedge e^{q}\right), \\
& =\sum_{i, p<q} \varphi_{p q} v^{i}\left(\delta_{i}^{p} e^{q}-\delta_{i}^{q} e^{p}\right), \\
& =\sum_{p<q} \varphi_{p q} v^{p} e^{q}-\sum_{p<q} \varphi_{p q} v^{q} e^{p}, \\
& =\sum_{p<q} \varphi_{p q} v^{p} e^{q}+\sum_{q<p} \varphi_{p q} v^{p} e^{q},
\end{aligned}
$$

where in the second sum of the last line we switched the names of the indices and for $p>q$, we set $\varphi_{p q}=-\varphi_{q p}$. Thus,

$$
v\lrcorner \varphi=\sum_{p, q} \varphi_{p q} v^{p} e^{q}=-\sum_{p, q} \varphi_{q p} v^{p} e^{q} .
$$

Continuing we obtain

$$
\begin{aligned}
(v\lrcorner \varphi) \wedge \omega & =\left(\sum_{p, q} \varphi_{p q} v^{p} e^{q}\right) \wedge\left(\sum_{k} \omega_{1 \ldots \widehat{k} \ldots . .} e^{1} \wedge \cdots \wedge \widehat{e}^{k} \wedge \cdots \wedge e^{n}\right), \\
& =\sum_{p, q, k} \varphi_{p q} v^{p} \omega_{1 \ldots \widehat{k} \ldots n} e^{q} \wedge e^{1} \wedge \cdots \wedge e^{k} \wedge \cdots \wedge e^{n}, \\
& =\sum_{p, q, k} \varphi_{p q} v^{p} \omega_{1 \ldots \widehat{k} \ldots . .} \delta^{q k}(-1)^{k-1} e^{1} \wedge \cdots \wedge e^{n}, \\
& =\sum_{p, q} \varphi_{p q} v^{p} \omega_{1 \ldots . . . . n}(-1)^{q-1} e^{1} \wedge \cdots \wedge e^{n},
\end{aligned}
$$

which confirms Equation (4.99).

Example 4.11. Continuing with the notation scheme of the previous examples, let $T=T_{1 \ldots n} e^{1} \wedge \cdots \wedge e^{n}$ be an $n$-form and consider the $n$-form $\left.\left(\iota_{T}(\omega)\right\lrcorner \varphi\right) \wedge \iota_{T}^{-1}(v)$ where $\iota_{T}$ is the isomorphism defined in Example 4.6. Using Equations (4.87) and (4.106),

$$
\begin{aligned}
\left.\left(\iota_{T}(\omega)\right\lrcorner \varphi\right) \wedge \iota_{T}^{-1}(v) & =\sum_{p, q} \varphi_{p q} \iota_{T}(\omega)^{p} \iota_{T}^{-1}(v)_{1 \ldots \widehat{q} \ldots n}(-1)^{q-1} e^{1} \wedge \cdots \wedge e^{n}, \\
& =\sum_{p, q} \varphi_{p q}(-1)^{p-1} \omega_{1 \ldots \widehat{p} \ldots n}(-1)^{q-1} v^{q}(-1)^{q-1} e^{1} \wedge \cdots \wedge e^{n}, \\
& =\sum_{p, q} \varphi_{p q}(-1)^{p-1} \omega_{1 \ldots \widehat{p} \ldots n} v^{q} e^{1} \wedge \cdots \wedge e^{n} .
\end{aligned}
$$

We conclude that

$$
\left.\left.\left(\iota_{T}(\omega)\right\lrcorner \varphi\right) \wedge \iota_{T}^{-1}(v)=-(v\lrcorner \varphi\right) \wedge \omega
$$


which is evidently independent of the $n$-form $T$.

4.IO. Pullback of Alternating Tensors. Let $A: \mathbf{W} \rightarrow \mathbf{V}$ be a linear mapping between vector spaces and $T \in \bigwedge^{r}\left(\mathbf{V}^{*}\right)$ an alternating $r$-tensor defined on $\mathbf{V}$. Then, $T$ induces an alternating $r$-tensor $A^{*}(T) \in \bigwedge^{r}\left(\mathbf{W}^{*}\right)$ by

$$
A^{*}(T)\left(w_{1}, \ldots, w_{r}\right)=T\left(A\left(w_{1}\right), \ldots, A\left(w_{r}\right)\right) .
$$

Let $\left\{e_{i}\right\}$ and $\left\{f_{p}\right)$ be bases in $\mathbf{W}$ and $\mathbf{V}$, respectively, so that $A\left(e_{i}\right)=\sum_{p} A_{i}^{p} f_{p}$, where $A_{i}^{p}$ is the matrix of $A$. Then,

$$
\begin{aligned}
A^{*}(T)_{\gamma} & =A^{*}\left(e_{\gamma_{1}}, \ldots, e_{\gamma_{r}}\right), \\
& =T\left(A\left(e_{\gamma_{1}}\right), \ldots, A\left(e_{\gamma_{r}}\right)\right), \\
& =T\left(\sum_{\mu_{1}} A_{\gamma_{1}}^{\mu_{1}} f_{\mu_{1}}, \ldots, \sum_{\mu_{r}} A_{\gamma_{r}}^{\mu_{r}} f_{\mu_{r}}\right), \\
& =\sum_{\mu} A_{\gamma_{1}}^{\mu_{1}} \cdots A_{\gamma_{r}}^{\mu_{r}} T\left(f_{\mu_{1}}, \ldots, f_{\mu_{r}}\right), \\
& =\sum_{\mu} A_{\gamma_{1}}^{\mu_{1}} \cdots A_{\gamma_{r}}^{\mu_{r}} T_{\mu_{1} \ldots \mu_{r}}, \\
& =\sum_{\mu,(\beta)} \varepsilon_{\mu_{1} \ldots \mu_{r}}^{\beta_{1} \ldots \beta_{r}} A_{\gamma_{1}}^{\mu_{1}} \ldots A_{\gamma_{r}}^{\mu_{r}} T_{\beta_{1} \ldots \beta_{r}} .
\end{aligned}
$$

Using Equation (4.6I)

$$
\begin{aligned}
& A^{*}(T \wedge S)\left(w_{1}, \ldots, w_{r+p}\right) \\
& \quad=T \wedge S\left(A\left(w_{1}\right), \ldots, A\left(w_{r+p}\right)\right), \\
& \quad=\frac{1}{r ! p !} \sum_{\pi}(-1)^{|\pi|} T\left(A\left(w_{\pi_{1}}\right), \ldots, A\left(w_{\pi_{r}}\right)\right) S\left(A\left(w_{\pi_{r+1}}\right), \ldots, A\left(w_{\pi_{r+p}}\right)\right), \\
& \quad=\frac{1}{r ! p !} \sum_{\pi}(-1)^{|\pi|} A^{*}(T)\left(w_{\pi_{1}}, \ldots, w_{\pi_{r}}\right) A^{*}(S)\left(w_{\pi_{r+1}}, \ldots, w_{\pi_{r+p}}\right), \\
& =A^{*}(T) \wedge A^{*}(S)\left(w_{1}, \ldots, w_{r+p}\right),
\end{aligned}
$$

and one obtains

$$
A^{*}(T \wedge S)=A^{*}(T) \wedge A^{*}(S) .
$$

4.II. Abstract Algebraic Cauchy Formula. Considering classical Cauchy formula as in Section 3.4, Equation (3.49) may be written in the standard form

$$
\phi=\frac{T(\tau)}{A}=\mathbf{J} \cdot \mathbf{n},
$$

where $\phi$ is the flux density induced by the flux vector $\mathbf{J}$ on the plane $\mathbf{H}$ determined by the normal unit vector $\mathbf{n}$. The metric independent version of (4.II3) may be written as follows.

Let $\mathbf{H}$ be an $\boldsymbol{r}$-dimensional affine subspace of the affine space $\mathbf{A}$ whose translation space is $\mathbf{U}$, an $r$-dimensional subspace of $\mathbf{V}$. Let $\iota_{\mathbf{U}}: \mathbf{U} \rightarrow \mathbf{V}$ be the natural 
inclusion. Then, the pullback $\iota_{\mathbf{U}}^{*}: \bigwedge^{r} \mathbf{V}^{*} \rightarrow \bigwedge^{r} \mathbf{U}^{*}$ given by

$$
\iota_{\mathbf{U}}^{*}(T)\left(v_{1}, \ldots, v_{r}\right)=T\left(\iota_{\mathbf{U}}\left(v_{1}\right), \ldots, \iota_{\mathbf{U}}\left(v_{r}\right)\right)=T\left(v_{1}, \ldots, v_{r}\right)
$$

is simply the restriction of the tensor $T$ to vectors in $\mathbf{U}$. We will use the notation $\rho_{\mathbf{U}}=\iota_{\mathbf{U}}^{*}$ for the restriction. Consider the case where $T$ is an $(n-1)$-alternating tensor and $\mathbf{H}$ is an $(n-1)$-dimensional hyperplane. Then, $T$ induces an $(n-1)$ alternating tensor $S$ on $\mathbf{U}$ given by

$$
S=\rho_{\mathbf{U}}(T)=\iota_{\mathbf{U}}^{*}(T),
$$

the abstract Cauchy formula.

\section{Manifolds and Their Basic Structure}

5.I. Atlases and Differentiable Manifolds. Let $\mathscr{S}$ be a Hausdorff topological space. A chart or a coordinate system in $\mathscr{S}$ is a mapping $\varphi: U \subset \mathscr{S} \rightarrow \mathbb{R}^{n}, U$ open in $\mathscr{S}$, such that $\varphi$ is a homeomorphism onto an open subset $V \subset \mathbb{R}^{n}$. In other words, $\varphi$ is an injective continuous mapping such that $\varphi^{-1}$, defined on $V=$ Image $U$, is also continuous. If $\varphi(x)=\left(x^{1}, \ldots, x^{n}\right)$, we say that $\left(x^{1}, \ldots, x^{n}\right)$ are the coordinates of $x$ under the chart $\varphi$ or that they represent $x$. We will often express this shortly by stating that $\left(x^{1}, \ldots, x^{n}\right)$ is a chart in $\mathscr{S}$. We will also denote a chart by $(\varphi, U)$. Such a chart does not necessarily exist for a general topological space.

An atlas is a collection $\left\{\left(\varphi_{a}, U_{a}\right)\right\}_{a \in A}$ of charts that cover $\mathscr{S}$. Assuming the collection is finite for simplicity, the atlas consists of $\left\{\varphi_{a}: U_{a} \rightarrow \mathbb{R}^{n}\right\}$, where $a=1, \ldots, A$, and $U_{a}$ are open in $\mathscr{S}$. Since the atlas covers $\mathscr{S}$, for every $x \in \mathscr{S}$, there is some $\varphi_{a}$ such that $x$ is contained in $U_{a}$. In case $U_{a} \cap U_{b} \neq \varnothing$, then, $\varphi_{b} \circ \varphi_{a}^{-1}$ defined on $\varphi_{a}\left\{U_{a} \cap U_{b}\right\}$ is the transformation of coordinates for the intersection $U_{a} \cap U_{b}$. Thus, an atlas, if one exists, endows $\mathscr{S}$ with an $n$-dimensional local Euclidean structure. An atlas $\left\{\varphi_{a}: U_{a} \rightarrow \mathbb{R}^{n}\right\}$ provides $\mathscr{S}$ with a structure of a differentiable manifold if the transformations of coordinates are smooth mappings. Thus, for $x \in U_{a} \cap U_{b}$, let $\left(x^{1}, \ldots, x^{n}\right)=\varphi_{a}(x)$ and $\left(x^{1^{\prime}}, \ldots, x^{n^{\prime}}\right)=\varphi_{b}(x)$ be the coordinates under the two charts. Then, the transformation of coordinates is of the form $x^{i^{\prime}}=x^{i^{\prime}}\left(x^{i}\right)$. For a differentiable structure, all such transformations and their inverses are thus required to be smooth. We will refer to this condition as the compatibility condition for the charts. In particular, it implies that the Jacobian matrices $\partial x^{i^{\prime}} / \partial x^{j}$ should be invertible. The atlas that induces the structure of a manifold need not be unique. In particular, an equivalent atlas may be obtained by adding a chart to an atlas provided it satisfies the compatibility condition with the existing charts.

A subset $\mathscr{A} \subset \mathscr{S}$ is referred to as an $m$-dimensional submanifold of $\mathscr{S}, m \leqslant n$, if for every $y \in \mathscr{A}$ there is a chart $\varphi: U \rightarrow \mathbb{R}^{n}$ such that $y \in U$, and for each $x \in U \cap \mathscr{A}$, the last $n-m$ coordinates of $x$, vanish, i.e., $\varphi(x)=\left(x^{1}, \ldots, x^{m}, 0, \ldots, 0\right)$. The collection of charts satisfying this condition induce and atlas and an $m$-dimensional manifold structure on $\mathscr{A}$.

The notion of a manifold may be extended to manifolds with boundaries. For a manifold with a boundary the co-domain of a chart is allowed to be in $\mathbb{R}^{n-}=\{x \in$ $\left.\mathbb{R}^{n} \mid x^{n} \leqslant 0\right\}$. For a manifold $\mathscr{S}$, a point $y \in \mathscr{S}$ is a boundary point if there is a chart $\varphi$ in a neighborhood $U$ of $y$ such that the $n$-th coordinate of $y$ vanishes, i.e., $\varphi(y)^{n}=0$. 
The fact that $\mathscr{S}$ as a given topology and the compatibility of charts imply that this definition is independent of the particular chart chosen. The collection of boundary points is the boundary $\partial \mathscr{S}$ of the manifold. By the definition of a submanifold, $\partial \mathscr{S}$ is a submanifold of $\mathscr{S}$ (see [Hir76, p. 29]).

Example 5.1. Bodies in Continuum Mechanics as Manifolds. As early as 1957, W. Noll [Nol59] has defined a body in continuum mechanics as a differentiable manifold that may be covered by a single chart. The various configurations of the body in a Euclidean physical space are viewed as charts on the abstract body object. The central point is that no preferred reference configuration is available in general and the differentiable structure of the body is exhibited through its configurations in space. In particular, on the abstract body manifold, notions such as straight lines, angles, and lengths are meaningless.

Example 5.2. Configuration spaces of mechanisms. The configuration spaces of various mechanisms and mechanical manipulators are differentiable manifolds. If only the kinematics of the mechanism is under consideration, there is no natural Riemannian metric on the configuration space. For example, the configuration space of a pendulum in the plane may be represented by the unit circle $S^{1}$. Equivalently, this configuration space may be represented by a circle of any radius, an ellipse, etc. For a mechanism having $m$ links connected in series by $m$ revolute joints, the configuration space may be represented by the $m$-torus $T^{m}=\left(S^{1}\right)^{m}$.

5.2. Mappings between Manifolds. Similarly to the way charts allow us to represent points on a manifold by real valued variables, local representatives of mappings provide the means for representing mappings between manifolds in terms of multi-variable mappings. Let $f: \mathscr{S} \rightarrow \mathscr{M}$ be a mapping between two manifolds. For a chart $\psi: V \rightarrow \mathbb{R}^{\operatorname{dim} \mathscr{M}}$ on $\mathscr{M}$ one can intersect the open set $f^{-1}\{V\}$ with the domains of charts in $\mathscr{S}$ and obtain a chart $\varphi: U \rightarrow \mathbb{R}^{\operatorname{dim} \mathscr{S}}$ in $\mathscr{S}$ such that

$$
\psi \circ f \circ \varphi^{-1}: \varphi(U) \longrightarrow \psi(V)
$$

which is of course a mapping between an open subset of $\mathbb{R}^{\operatorname{dim} \mathscr{S}}$ and $\mathbb{R}^{\operatorname{dim} \mathscr{M}}$. As such, the differentiability properties of $f$ are well defined. The mapping $\psi \circ f \circ \varphi^{-1}$ is referred to as a local representative of $f$ relative to the charts under consideration. If $x^{i}$ and $y^{j}$ are the local coordinates under $\varphi$ and $\psi$, respectively, then the local representative is of the form $y^{j}=y^{j}\left(x^{i}\right)$.

A mapping $f: \mathscr{S} \rightarrow \mathscr{M}$ is said to be smooth if its local representatives are smooth. By the compatibility conditions, this property is independent of the local representatives chosen. A smooth mapping between two manifolds is an immersion if for each local representative $\psi \circ f \circ \varphi^{-1}$, the derivative $D\left(\psi \circ f \circ \varphi^{-1}\right)$ is an injective linear mapping at each point in the domain. Thus, in terms of local coordinates, the matrix $\left[\partial y^{j} / \partial x^{i}\right]$ is of $\operatorname{rank} \operatorname{dim} \mathscr{S}$. The mapping is a submersion if the derivatives of local representatives are surjective so that $\left[\partial y^{j} / \partial x^{i}\right]$ is of $\operatorname{rank} \operatorname{dim} \mathscr{M}$.

An embedding is an immersion whose image is homeomorphic to $\mathscr{S}$. It can be shown that the image of an embedding is a submanifold of $\mathscr{M}$. A diffeomorphism is an invertible mapping $f: \mathscr{S} \rightarrow \mathscr{M}$ which is smooth and whose inverse $f^{-1}$ : $\mathscr{M} \rightarrow \mathscr{S}$ is also smooth. In case there is a diffeomorphism between $\mathscr{S}$ and $\mathscr{M}$ 
one says that the two manifolds are diffeomorphic. This means that $\mathscr{S}$ and $\mathscr{M}$ are equivalent in the category of differentiable manifolds. For example, if $g: \mathscr{S} \rightarrow \mathscr{M}$ is an embedding, $\mathscr{S}$ is diffeomorphic with its image.

Example 5.3. Configurations of bodies. In a general geometric setting of continuum mechanics, e.g., [Seg86], both a body $B$ and the physical space $\mathscr{S}$ are represented by differentiable manifolds. Traditionally, one assumes in continuum mechanics that the principle of material impenetrability holds. This implies that the deformation gradient is injective at each point and that the configuration mapping is injective. In the framework of mechanics on differentiable manifolds, the impenetrability principle together with the requirement for differentiability imply that a configuration should be an embedding of the body manifold in the space manifold.

Example 5.4. In the continuum mechanics of generalized media ${ }^{2}$ in addition to the variable specifying the location in space of the various material points, the configuration of a body is described by additional degrees of freedom (sometimes referred also as order parameters) describing the micro-configuration of a material point (see [Cap89, CMo2]). These variables usually assume values on manifolds different than the space manifold so that even in the case where the physical space is modeled as a Euclidean space, the micro configuration of a point is valued on a manifold devoid of a natural metric structure. As a simple example, the void fraction of a porous material may be described by a real number so that the space of micro configuration $\mathscr{M}$ may be modeled by the segment $[0,1] \in \mathbb{R}$. Clearly, this parametrization is arbitrarily chosen and other charts on $\mathscr{M}$ may be given. Thus, in general, the micro-configuration of a body $B$ is given by a mapping $m: B \rightarrow \mathscr{M}$.

5.3. Tangent Vectors and the Tangent Bundle. For the example where a manifold models the configuration space of a mechanical system, a tangent vector is the mathematical object that represents a virtual velocity. In particular, let a manifold $\mathscr{S}$ model the physical space so that $\mathscr{S}$ is the configuration space of a particle moving in space. Then, a tangent vector represents the velocity of the particle. For the example where a manifold models a body in continuum mechanics, a tangent vector originating from $X \in \mathscr{S}$ may be thought of as the mathematical object that represents a point infinitesimally close to $X$. The interpretation of a tangent vector as a virtual velocity fits the formal definition below.

A curve in the manifold $\mathscr{S}$ is a smooth mapping $c:(-\epsilon, \epsilon) \rightarrow \mathscr{S}$, where $(-\epsilon, \epsilon)$ is some interval in $\mathbb{R}$ containing zero. For the interpretation of a manifold as a configuration space of a mechanical system, a curve represents a motion of the system where the variable in $\mathbb{R}$ represents time. We say that $c$ is a curve at $x \in \mathscr{S}$ if $c(0)=x$. The local representative of a curve is of the form $x^{i}=x^{i}(t)$.

Let $c_{1}$ and $c_{2}$ be two curves at $x \in \mathscr{S}$. We say that the two curves are tangent at $x$ if their local representatives $x_{1}^{i}(t)$ and $x_{2}^{i}(t)$ under some chart $\varphi: U \rightarrow \mathbb{R}^{n}$ defined

\footnotetext{
${ }^{2}$ This includes media referred to by various authors as bodies with microstructure, bodies with substructure, Cosserat media, multi-polar media, etc.
} 
in a neighborhood of $x$ satisfy

$$
\left.\frac{\mathrm{d}}{\mathrm{d} t} x_{1}^{i}(t)\right|_{t=0}=\left.\frac{\mathrm{d}}{\mathrm{d} t} x_{2}^{i}(t)\right|_{t=0}, \quad i=1, \ldots, n .
$$

Thus, the two curves are tangent at $x$ if their local representatives are tangent as curves in $\mathbb{R}^{n}$. (Note that tangency implies that the derivatives are equal and not merely belong to the same one dimensional space.) While the values of the derivatives depend on the chart chosen, the property of tangency is independent of the chart. If $\varphi^{\prime}$ is another chart in a neighborhood of $x$ such that the local representative of a curve $c$ under $\varphi^{\prime}$ is given by $x^{i^{\prime}}=x^{i^{\prime}}(t)$, then,

$$
\left.\frac{\mathrm{d}}{\mathrm{d} t} x_{1}^{i^{\prime}}(t)\right|_{t=0}=\left.\frac{\partial x^{i^{\prime}}}{\partial x^{i}}\left(x^{i}(0)\right) \frac{\mathrm{d}}{\mathrm{d} t} x_{1}^{i}(t)\right|_{t=0}=\left.\frac{\mathrm{d}}{\mathrm{d} t} x_{2}^{i^{\prime}}(t)\right|_{t=0} .
$$

While one cannot associate naturally a unique element of $\mathbb{R}^{n}$ with the derivative of a curve at $x$, we identify the derivative with the collection of all curves that are tangent to one another at $x$. In more precise terms, the derivative - a tangent vector at $x$-is an equivalence class of curves under the equivalence relation of tangency of curves at $x$. In other words, for a tangent vector $v$ at $x$, any curve $c$ in this class may represent $v$. Any other curve that is tangent to $c$ at $x$ is equally qualified to represent $v$. In the sequel, we will use a superimposed dot to denote the derivative of a curve in $\mathbb{R}^{n}$ so that the tangency condition may be written in the form $\dot{x}_{1}^{i}(0)=\dot{x}_{2}^{i}(0)$. For a given tangent vector $v$ at $x$ and a chart $\varphi$ in a neighborhood of $x,\left(\dot{x}^{1}(0), \ldots, \dot{x}^{n}(0)\right) \in \mathbb{R}^{n}$ represents $v$. The vector $\left(\dot{x}^{1}(0), \ldots, \dot{x}^{n}(0)\right) \in \mathbb{R}^{n}$ depends on the chart $\varphi$ but is independent of the particular curve used to represent the tangent vector to the manifold at $x$. The foregoing procedure enables one to generalize the notion of a velocity of a particle traveling in a Euclidean space to the case of a motion in some abstract space manifold and to mechanical systems whose motions take place in configuration manifolds.

The tangent space $T_{x} \mathscr{S}$ to the manifold $\mathscr{S}$ at $x$ is the collection of the various tangent vectors at $x$. Given a chart $\varphi$ in a neighborhood $U$ of $x \in \mathscr{S}$, consider the mapping

$$
T_{x} \varphi: T_{x} \mathscr{S} \longrightarrow \mathbb{R}^{n}, \quad T_{x} \varphi(v)=\left.\frac{\mathrm{d}}{\mathrm{d} t} c(t)\right|_{t=0}=\left(\dot{x}^{1}(0), \ldots, \dot{x}^{n}(0)\right),
$$

where $c$ is any curve that represents the tangent vector $v$. This mapping is a bijection since for a given vector $\left(v^{1}, \ldots, v^{n}\right) \in \mathbb{R}^{n}$, any curve at $x$ whose restriction to a small neighborhood of $x$ is of the form $c(t)=\varphi^{-1}\left(\varphi(x)+t\left(v^{1}, \ldots, v^{n}\right)\right)$ will represent a tangent vector $v \in T_{x} \mathscr{S}$ such that $T_{x} \varphi(v)=\left(v^{1}, \ldots, v^{n}\right)$. The bijection $T_{x} \varphi$ induces an $n$-dimensional vector space structure on $T_{x} \mathscr{S}$ by which $T_{x} \varphi$ becomes a vector space isomorphism. That is, for $v, v^{\prime} \in T_{x} \mathscr{S}$ and $a, b \in \mathbb{R}$, we define

$$
a v+b v^{\prime}=\left(T_{x} \varphi\right)^{-1}\left(a T_{x} \varphi(v)+b T_{x} \varphi\left(v^{\prime}\right)\right) .
$$

Let $\mathbf{e}_{i}$ be the $i$-th natural base vector of $\mathbb{R}^{n}$ for which all the components are zero except for the $i$-th component which is equal to one. Then, the isomorphism $T_{x} \varphi$ induces a chart dependent basis $\left\{e_{i}\right\}_{i=1}^{n}$ for $T_{x} \mathscr{S}$ by $e_{i}=\left(T_{x} \varphi\right)^{-1}\left(\mathbf{e}_{i}\right)$. It is also 
customary to use the notation (without justification at this point)

$$
\frac{\partial}{\partial x^{i}}:=e_{i}
$$

Thus,

$$
v=\sum_{i} v^{i} e_{i}=\sum_{i} v^{i} \frac{\partial}{\partial x^{i}},
$$

where by the definition of $T_{x} \varphi, v^{i}$ are the components of $T_{x} \varphi(v)$. It is emphasized again that the components $v^{i}$ and base vectors depend on the choice of chart and are not "invariant" quantities.

It is noted that for two distinct points $x, x^{\prime} \in \mathscr{S}$, the two tangent spaces $T_{x} \mathscr{S}$ and $T_{x^{\prime}} \mathscr{S}$ are both isomorphic with $\mathbb{R}^{n}$ but there is no natural, chart independent, isomorphism between them.

If $\varphi$ and $\varphi^{\prime}$ are charts in a neighborhood of $x \in \mathscr{S}$ and the local representatives of a curve $c$ relative to the two charts are given in the form $\left(x^{1}(t), \ldots, x^{n}(t)\right)$, and $\left(x^{1^{\prime}}(t), \ldots, x^{n^{\prime}}(t)\right)$, then, the transformation rule for the components of tangent vectors is

$$
T_{x} \varphi^{\prime} \circ\left(T_{x} \varphi\right)^{-1}: \mathbb{R}^{n} \longrightarrow \mathbb{R}^{n}
$$

given by

$$
\dot{x}^{i^{\prime}}=\frac{\partial x^{i^{\prime}}}{\partial x^{i}} \dot{x}^{i}
$$

In other words, denoting the derivative of the transformation of coordinates by $D\left(\varphi^{\prime} \circ \varphi^{-1}\right)$ one has

$$
T_{x} \varphi^{\prime} \circ\left(T_{x} \varphi\right)^{-1}=D\left(\varphi^{\prime} \circ \varphi^{-1}\right)(\varphi(x)) .
$$

The tangent bundle $T \mathscr{S}$ is the union of the tangent spaces at the various points of the manifold, i.e.,

$$
T \mathscr{S}=\bigcup_{x \in \mathscr{S}} T_{x} \mathscr{S}
$$

There is a natural mapping

$$
\tau: T \mathscr{S} \longrightarrow \mathscr{S}, \quad \tau(v)=x \text {, if } x \in T_{x} \mathscr{S} .
$$

Clearly, $\tau$ is a surjection. In particular, $\tau^{-1}\{x\}=T_{x} \mathscr{S}$.

We now observe that an atlas $\left\{\varphi_{a}: U_{a} \rightarrow \mathbb{R}^{n}\right\}$ on $\mathscr{S}$, induces naturally a $2 n$ dimensional atlas on $T \mathscr{S}$. For each $a$, consider the mapping

$$
\Phi_{a}: \tau^{-1}\left\{U_{a}\right\} \longrightarrow \mathbb{R}^{2 n}, \quad \Phi_{a}(v)=\left(\varphi_{a}(\tau(v)), T_{\tau(v)} \varphi_{a}(v)\right) .
$$

Evidently, the sets $\tau^{-1}\left\{U_{a}\right\}$ cover $T \mathscr{S}$. For the two charts $\left(\varphi_{a}, U_{a}\right)$ and $\left(\varphi_{b}, U_{b}\right)$, $U_{a} \cap U_{b} \neq \varnothing$, the transformation of coordinates $\Phi_{b} \circ \Phi_{a}^{-1}: \mathbb{R}^{2 n} \rightarrow \mathbb{R}^{2 n}$ is given by

$$
\Phi_{b} \circ \Phi_{a}^{-1}\left(x^{1}, \ldots, x^{n}, v^{1}, \ldots, v^{n}\right)=\left(x^{1^{\prime}}, \ldots, x^{n^{\prime}}, v^{1^{\prime}}, \ldots, v^{n^{\prime}}\right),
$$

where by (5.9)

$$
\left(x^{1^{\prime}}, \ldots, x^{n^{\prime}}\right)=\left(\varphi_{b} \circ \varphi_{a}^{-1}\right)\left(x^{1}, \ldots, x^{n}\right), \quad v^{i^{\prime}}=\sum_{i} \frac{\partial x^{i^{\prime}}}{\partial x^{i}} v^{i} .
$$


It is noted that the transformation rule for the components $v^{i}$-the principal components of the element $v \in T \mathscr{S}$-is linear. In addition, we observe that if an affine space $\mathbf{A}$ with a translation vector space $\mathbf{V}$ is regarded as a manifold, the tangent space at each point may be identified with $\mathbf{V}$ and the tangent bundle may be identified with $\mathbf{A} \times \mathbf{V}$. In particular, the tangent bundle of a vector space $\mathbf{V}$ is identified with $\mathbf{V} \times \mathbf{V}$.

5.4. Fiber bundles and Vector Bundles. A chart $\Phi$ on the tangent bundle $T \mathscr{S}$ determined by a chart $\varphi$ on an open set $U \subset \mathscr{S}$ induces on $\tau^{-1}\{U\}$ a local structure of a Cartesian product $U \times \mathbb{R}^{n}$. A different chart on that neighborhood will also induce such a product structure that will preserve the vector space structure of each tangent space. Such a structure on a manifold occurs in various examples. The local structure of a Cartesian product is the property that makes a manifold a fiber bundle, where $T \mathscr{S}$ is the only example we have so far. Roughly, a fiber bundle may be thought of as the manifold obtained by smoothly attaching a copy of a manifold $\mathscr{F}$ called the typical fiber of the bundle, to each point of a manifold $\mathscr{S}$ called the base manifold.

5.4.I. Fiber bundles. A fiber bundle structure on a manifold $\mathscr{M}$ having a base manifold $\mathscr{S}$ consists of a projection mapping $\pi: \mathscr{M} \rightarrow \mathscr{S}$ and a fiber bundle atlas that have the following properties. For all $x \in \mathscr{S}, \pi^{-1}\{x\}$ are diffeomorphic to a $d$ dimensional manifold $\mathscr{F}$, called the typical fiber of the fiber bundle. The fiber bundle atlas consists of a collection $\left\{\left(\Phi_{a}, \varphi_{a}, U_{a}\right)\right\}$ such that $\left\{\left(\varphi_{a}, U_{a}\right)\right\}$ is an atlas of $\mathscr{S}$ and each $\Phi_{a}: \pi^{-1}\left\{U_{a}\right\} \rightarrow U_{a} \times \mathscr{F}$, called a local trivialization, is a diffeomorphism such that

$$
\operatorname{pr}_{1} \circ \Phi_{a}(m)=\pi(m), \quad \text { for every } m \in \pi^{-1}\left\{U_{a}\right\} .
$$

Here and in the sequel, $\mathrm{pr}_{1}$ and $\mathrm{pr}_{2}$ denote the natural projections on the factors of a Cartesian product. We will refer to $\mathscr{M}$ is the total space of the fiber bundle and will denote the fiber bundle by $\pi: \mathscr{M} \rightarrow \mathscr{S}$. Sometimes we will also refer to either $\mathscr{M}$ or $\pi$ as the fiber bundle for short. We will also use $\pi$ to denote the projection of a generic fiber bundle. The manifold $\mathscr{M}_{x}:=\pi^{-1}\{x\}$ is usually referred to as the fiber of the bundle at $x$.

For a nonempty intersection $U_{a} \cap U_{b}$ one has the transformation

$$
\Phi_{b} \circ \Phi_{a}^{-1}:\left(U_{a} \cap U_{b}\right) \times \mathscr{F} \longrightarrow\left(U_{a} \cap U_{b}\right) \times \mathscr{F}
$$

where by (5.I6) $\Phi_{b} \circ \Phi_{a}^{-1}(x, f)=\left(x, f^{\prime}\right)$. It follows that for any $x \in U_{a} \cap U_{b}$ there is a diffeomorphism $\Phi_{a b}(x): \mathscr{F} \rightarrow \mathscr{F}$ such that $f^{\prime}=\Phi_{a b}(x)(f)$. Thus, $\Phi_{b} \circ \Phi_{a}^{-1}(x, f)=\left(x, \Phi_{a b}(x)(f)\right)$.

Let $\left\{\left(\psi_{l}, V_{l}\right)\right\}_{l=1}^{L}$ be an atlas on $\mathscr{F}$. Then, given a point $m \in \mathscr{M}$, there is a chart $\varphi_{a}: U_{a} \rightarrow \mathbb{R}^{n}$ such that $\operatorname{pr}_{1}(\Phi(m))=\pi(m) \in U_{a}$ and a chart $\psi_{l}: V_{l} \rightarrow \mathbb{R}^{d}$ such that $\operatorname{pr}_{2}(\Phi(m)) \in V_{l}$. Let $\bar{U}_{a}=\operatorname{Image} \varphi_{a}, \bar{V}_{l}=$ Image $\psi_{l}$. Then on $R_{a l}=$ $\Phi_{a}^{-1}\left\{\varphi_{a}^{-1}\left\{\bar{U}_{a}\right\} \times \psi^{-1}\left\{\bar{V}_{l}\right\}\right\} \subset \mathscr{M}$, an open neighborhood of $m$, one has the chart

$$
\Psi_{a l}:=\left(\varphi_{a} \circ \mathrm{pr}_{1}, \psi_{l} \circ \mathrm{pr}_{2}\right) \circ \Phi_{a}: R_{a l} \longrightarrow \mathbb{R}^{n} \times \mathbb{R}^{d} .
$$


Let $\left(z^{1}, \ldots, z^{d}\right)$ be the local coordinates in $\mathbb{R}^{d}$, then, under the chart $\left(\Psi_{a l}, R_{a l}\right)$, elements in the neighborhood of $m$ will be represented in the form $\left(x^{1}, \ldots, x^{n}, z^{1}, \ldots, z^{d}\right)$ and $\pi$ is represented locally by $\left(x^{1}, \ldots, x^{n}, z^{1}, \ldots, z^{d}\right) \mapsto\left(x^{1}, \ldots, x^{n}\right)$.

A fiber bundle is trivial if it has a global structure of a Cartesian product, i.e., $\mathscr{M}=\mathscr{S} \times \mathscr{F}$.

5.4.2. Sections of Fiber Bundles and Fiber Bundle Morphisms. A section of a fiber bundle is a smooth mapping $w: \mathscr{S} \rightarrow \mathscr{M}$ satisfying $\pi(w(x))=x$. Thus, a section assigns to each point $x$ an element of the fiber $\mathscr{M}_{x}$-a generalization of the notion of a field. A section $w$ of the fiber bundle is represented locally by $\left(x^{1}, \ldots, x^{n}\right) \longmapsto\left(x^{1}, \ldots, x^{n}, z^{1}\left(x^{i}\right), \ldots, z^{d}\left(x^{i}\right)\right)=\left(x^{1}, \ldots, x^{n}, w^{1}\left(x^{i}\right), \ldots, w^{d}\left(x^{i}\right)\right)$,

where $w^{1}, \ldots, w^{d}$ are $d$ real valued functions defined on $\mathbb{R}^{n}$ representing the principal components of the mapping $w$.

A fiber bundle morphism is a mapping between two fiber bundles that preserves the bundle structure. More precisely, a fiber bundle morphism $\left(f, f_{0}\right)$ between the fiber bundles $\pi_{1}: \mathscr{M}_{1} \rightarrow \mathscr{S}_{1}$ and $\pi_{2}: \mathscr{M}_{2} \rightarrow \mathscr{S}_{2}$ consists of a smooth map $f: \mathscr{M}_{1} \rightarrow$ $\mathscr{M}_{2}$ and a smooth map $f_{0}: \mathscr{S}_{1} \rightarrow \mathscr{S}_{2}$, called the base mapping, such that $\pi_{2} \circ f=$ $f_{0} \circ \pi_{1}$. Thus, if $m$ and $m^{\prime}$ belong to the same fiber, say $\mathscr{M}_{1 x}$, i.e., $\pi_{1}(m)=\pi_{1}\left(m^{\prime}\right)=x$, then, $\pi_{2}(f(m))=\pi_{2}\left(f\left(m^{\prime}\right)\right)=f_{0}(x)$ so that both $f(m)$ and $f\left(m^{\prime}\right)$ belong to the same fiber $\mathscr{M}_{2 f_{0}(x)}=\pi_{2}^{-1}\left\{f_{0}(x)\right\}$. In other words, the restriction $f_{x}$ of $f$ to the fiber $\mathscr{M}_{1 x}$ maps it into the fiber $\mathscr{M}_{2 f_{0}(x)}$.

Example 5.5. In Galilean mechanics, time is an absolute property of an event while the location in space depends on the frame where the event is observed. Thus, while the copies of space at distinct time instances are diffeomorphic, there is no natural way to specify a fixed location in space in distinct time instances. The geometrical structure suitable for the description of Galilean spacetime is that of a fiber bundle $\pi: \mathscr{M} \rightarrow \mathscr{T}$. The base manifold $\mathscr{T}$ is the time manifold and the fiber bundle projection assigns to each event $e \in \mathscr{M}$ a unique time. The typical fiber is some manifold $\mathscr{S}$ and at each time $t \in \mathscr{T}, \pi^{-1}(t)$ is diffeomorphic to $\mathscr{S}$. A physical frame is a (global) trivialization $\Phi: \mathscr{M} \rightarrow \mathscr{T} \times \mathscr{S}$ (see Section 9).

In various examples of fiber bundles, the typical fiber has additional structure that is preserved by the local trivializations. A typical example is a vector bundle for which the typical fiber is a vector space.

5.4.3. Vector Bundles. A vector bundle $\pi: W \rightarrow \mathscr{S}$ is a fiber bundle for which the typical fiber is a $d$-dimensional vector space $\mathbf{V}$. Consider the nonempty intersection $U_{a} \cap U_{b}$ of the domain of charts and the transformation $\Phi_{a b}(x)$ defined above by $\left(x, \Phi_{a b}(x)(v)\right)=\Phi_{b} \circ \Phi_{a}^{-1}(x, v)$ for $v \in \mathbf{V}$. It is required that for every $x \in U_{a} \cap U_{b}$, $\Phi_{a b}(x)$ be a linear transformation of $\mathbf{V}$. As a result of the definition, the mapping

$$
\Phi_{a x}:=\left.\Phi_{a}\right|_{W_{x}}: W_{x} \longrightarrow \mathbf{V} .
$$

is an isomorphism of the fiber $\pi^{-1}\{x\}=W_{x}$ with $\mathbf{V}$. Thus, $\Phi_{a x}$ induces a vector bundle structure on $W_{x}$. The requirement that the various $\Phi_{a b}$ are linear implies 
that this vector space structure is independent of the choice of a chart. It is noted that no natural isomorphism between the two spaces is available in general.

A basis $\left\{\mathbf{g}_{1}, \ldots, \mathbf{g}_{d}\right\}$ of $\mathbf{V}$, induces at each $x \in U_{a}$ a basis $\left\{g_{1}, \ldots, g_{d}\right\}$ of $W_{x}$ by $g_{k}=\Phi_{a x}^{-1}\left(\mathbf{g}_{k}\right)$. Thus, an element $w$ of the vector bundle may be represented in the forms $\left(x_{1}, \ldots, x_{n}, w_{1}, \ldots, w_{d}\right)$ and $w=\sum_{k} w^{k} g_{k}$.

The only example of a vector bundle we have so far is the tangent bundle. Naturally, a section of a vector bundle, and in particular, a section of the tangent bundle is referred to as a vector field. Since a coordinate system induces locally the bases $e_{i}=\partial / \partial x^{i}$ in the various spaces $T_{x} \mathscr{S}$, a vector field $w$ in $T \mathscr{S}$ is represented locally in the form

$$
\sum_{i} w^{i}\left(x^{j}\right) \frac{\partial}{\partial x^{i}} .
$$

A vector bundle morphism between the vector bundles $\pi_{1}: W_{1} \rightarrow \mathscr{S}_{1}$ and $\pi_{2}: W_{2} \rightarrow$ $\mathscr{S}_{2}$ is a fiber bundle morphism $\left(f, f_{0}\right)$ such that for each $x \in \mathscr{S}_{1}$ the restriction $f_{x}=\left.f\right|_{x}$ is a linear mapping of $W_{1 x}$ into $W_{2} f_{0}(x)$.

Consider the sections of a vector bundle. Using the vector space structure of each fiber, linear combinations of sections of a vector bundle are defined by

$$
\left(a w+a^{\prime} w^{\prime}\right)(x)=a w(x)+a^{\prime} w^{\prime}(x) .
$$

This definition endows the collection of sections of a vector bundle with the structure of a vector space. The infinite dimensional space of smooth sections of a fiber bundle $\pi: \mathscr{M} \rightarrow \mathscr{S}$ is denoted by $C^{\infty}(\pi)$ or $C^{\infty}(\mathscr{M})$. Thus, for the case of a vector bundle, $C^{\infty}(\pi)$ is a vector space.

5.4.4. Tangent mappings. The notion of the derivative of a mapping between two multi-variable spaces can be generalized to manifolds. Given a smooth mapping $f: \mathscr{S}_{1} \rightarrow \mathscr{S}_{2}, \operatorname{dim} \mathscr{S}_{1}=n, \operatorname{dim} \mathscr{S}_{2}=n^{\prime}$, the tangent mapping is a vector bundle morphism $T f: T \mathscr{S}_{1} \rightarrow T \mathscr{S}_{2}$, defined as follows. Let $v$ be a tangent vector at $x \in \mathscr{S}_{1}$ and let the curve $c: \mathbb{R} \rightarrow \mathscr{S}_{1}$ be a curve representing $v$. Then, $f \circ c: \mathbb{R} \rightarrow \mathscr{S}_{2}$ is a curve at $f(x)$ and $T f(v) \in T_{f(x)} \mathscr{S}_{2}$ is defined to be the tangent vector that $f \circ c$ induces. To see the sense in which the tangent mapping generalizes the derivative of a multi-variable mapping, consider local coordinates $x^{i}$ in a neighborhood of $x$ and the local coordinates $y^{k}$ in a neighborhood of $f(x)$. Then, the vector $v$ is represented by $\left(v^{1}, \ldots, v^{n}\right)=\left(\dot{x}^{1}(0), \ldots, \dot{x}^{n}(0)\right)$, where $x^{i}(t)$ represent the curve $c$. Let a local representative of $f$ be given by the functions $y^{k}=f^{k}\left(x^{i}\right)$ so that $f \circ c$ is represented by $y^{k}(t)=f^{k}\left(x^{i}(t)\right)$. It follows that $T f(v)$ is represented by $\left(u^{1}, \ldots, u^{n^{\prime}}\right)=\left(\dot{y}^{1}(0), \ldots, \dot{y}^{n^{\prime}}(0)\right)$, given as

$$
u^{k}=\sum_{i} \frac{\partial f^{k}}{\partial x^{i}} v^{i},
$$

where we omitted the indication of location where the derivatives are evaluated. Thus, the restriction $T_{x} f$ of the tangent mapping to the tangent space $T_{x} \mathscr{S}_{1}$, is represented by the Jacobian matrix of the partial derivatives of the local representatives of $f$. We conclude that the tangent mapping is represented locally in the 
form

$$
\left(x^{1}, \ldots, x^{n}, v^{1}, \ldots, v^{n}\right) \longmapsto\left(f^{1}\left(x^{i}\right), \ldots, f^{n^{\prime}}\left(x^{i}\right), \sum_{i} \frac{\partial f^{1}}{\partial x^{i}} v^{i}, \ldots, \sum_{i} \frac{\partial f^{n^{\prime}}}{\partial x^{i}} v^{i}\right) .
$$

Let $f_{1}: \mathscr{S}_{1} \rightarrow \mathscr{S}_{2}$ and $f_{2}: \mathscr{S}_{2} \rightarrow \mathscr{S}_{3}$ be smooth mappings. From the local representation of the a tangent mapping above and using the chain rule of multivariable calculus, it follows that

$$
T_{x}\left(f_{2} \circ f_{1}\right)=T_{f_{1}(x)} f_{2} \circ T_{x} f_{1}, \quad T\left(f_{2} \circ f_{1}\right)=T f_{2} \circ T f_{1} .
$$

Example 5.6. For a formulation of continuum mechanics in which both the body and space are modeled as general differentiable manifolds, as in Example 5.3, the traditional deformation gradient should be replaced by the tangent to the configuration mapping between the body and space manifolds. Thus, it can no longer be represented as a tensor. The fact that the deformation gradient is a "two point tensor" is indicative of the fact that it is a vector bundle morphism between the tangent bundles of two different manifolds. Even in the case where a body is an abstract manifold and space is a Euclidean 3-dimensional space, the derivative of a configuration should be represented by the tangent mapping rather than a tensor.

5.4.5. Pullback ofvectorbundles. Let $\pi: W \rightarrow \mathscr{S}$ be a vector bundle whose typical fiber is $\mathbf{V}$, and for a manifold $\mathscr{M}$, let $f: \mathscr{M} \rightarrow \mathscr{S}$ be a smooth mapping. The mapping $f$ determines a vector bundle

$$
f^{*}(\pi): f^{*}(W) \longrightarrow \mathscr{M}
$$

over $\mathscr{M}$ called the pullback of the vector bundle $\pi$ by $f$. It is defined as follows. For each $m \in \mathscr{M}$

$$
f^{*}(\pi)^{-1}\{m\}=f^{*}(W)_{m}=\pi^{-1}\{f(m)\}=W_{f(m)},
$$

i.e., the fiber over $m \in \mathscr{M}$ of the pullback fiber bundle is identified with the fiber at $f(m)$. Thus, the typical fiber of $f^{*}(\pi)$ is also $\mathbf{V}$. In addition, one has a natural vector bundle morphism $\left(\pi^{*}(f), f\right)$, where the mapping $\pi^{*}(f): \pi^{*}(W) \rightarrow W$ is defined by the requirement that for $u \in f^{*}(W)_{m}$, i.e., $m=f^{*}(\pi)(u), \pi^{*}(f)(u)$ is the unique element in $W_{f(m)}$ which is identified with $u$ under the construction of the pullback vector bundle.

If $w: \mathscr{S} \rightarrow W$ is a section of the vector bundle $\pi$, one has an induced section $f_{\pi}^{*}(w): \mathscr{M} \rightarrow f^{*}(\pi)$ of the pullback bundle defined by $f_{\pi}^{*}(w)(x)=w(f(x))$.

Example 5.7. Virtual velocity fields. Again we consider continuum mechanics on manifolds. Let the embedding $\kappa: B \rightarrow \mathscr{S}$ be a configuration of a body in space. A virtual velocity field (or a virtual displacement field) $w$ assigns to each material point $X \in B$ a tangent vector at $\kappa(X)$, i.e., it is a mapping $w: B \rightarrow T \mathscr{S}$ such that $\tau \circ w=\kappa(\tau$ : $T \mathscr{S} \rightarrow \mathscr{S}$ being the tangent bundle bundle projection). Since $\kappa$ is an embedding, its inverse $\kappa^{-1}$ : Image $\kappa \rightarrow B$ is well defined and the virtual displacement field induces a section $w \circ \kappa^{-1}:$ Image $\kappa \rightarrow T \mathscr{S}$. Clearly, $\tau \circ w \circ \kappa^{-1}(x)=x$ for all $x \in \operatorname{Image} \kappa$. The section $w \circ \kappa^{-1}$ is traditionally referred to as the Eulerian version of the velocity field. 
Alternatively, consider the pullback of the tangent bundle $\kappa^{*}(\tau): \kappa^{*}(T \mathscr{S}) \rightarrow B$ over the body. The velocity field $w$ induces a section $u: B \rightarrow \kappa^{*}(T \mathscr{S})$ by setting $u(X)$ to be the unique element of $\kappa^{*}(T \mathscr{S})_{X}$ which is equal to $w(X) \in T_{\kappa(X)} \mathscr{S}$ by the construction of the pullback bundle. It is noted that while the section $u$ is defined whether $\kappa$ is invertible or not, the Eulerian vector field cannot be defined if $\kappa$ is not invertible. Thus, it is convenient to regard a virtual velocity field as a section of the pullback $\kappa^{*}(T \mathscr{S})$.

In the case of bodies with microstructure described in Example 5.4, a virtual velocity field at the micro configuration $m: B \rightarrow \mathscr{M}$ will be a section $w$ of $m^{*}(T \mathscr{M})$ or equivalently, a mapping $w: B \rightarrow T \mathscr{M}$ with $\tau_{\mathscr{M}} \circ w=m$. It is noted that as a micro-configuration is not expected to be an embedding, there is no Eulerian version of micro virtual velocity field. Thus, for all the situations described above, a virtual velocity field at a given configuration of a body may be modeled as a section of a vector bundle.

5.5. Dual Vector Bundles and the Cotangent Bundle. Given a vector bundle $\pi: W \rightarrow \mathscr{S}$ with typical fiber $\mathbf{V}$, a vector bundle $\pi: W^{*} \rightarrow \mathscr{S}$, whose typical fiber is the dual $\mathbf{V}^{*}$ is defined naturally. Note that we use the same generic notation, $\pi$, for the dual bundle projection mapping unless a clear distinction has to be made. Clearly, the mapping

$$
\left(\Phi_{a x}^{*}\right)^{-1}: W_{x}^{*} \longrightarrow \mathbf{V}^{*}
$$

(see Equation (5.20)) determines the required isomorphism for each $x \in U_{a}$. As the basis $\left\{\mathbf{g}_{1}, \ldots, \mathbf{g}_{d}\right\}$ of $\mathbf{V}$ induces a dual basis $\left\{\mathbf{g}^{1}, \ldots, \mathbf{g}^{d}\right\}$ of $\mathbf{V}^{*}$, a dual basis $\left\{g^{1}, \ldots, g^{d}\right\}, g^{k}=\Phi_{a x}^{*}\left(\mathbf{g}^{k}\right)$ will be defined in $W_{x}^{*}$. An element $\phi \in W_{x}^{*}$ will be represented in the form $\left(x^{1}, \ldots, x^{n}, \phi_{1}, \ldots, \phi_{d}\right)$ or $\phi=\sum_{k} \phi_{k} g^{k}$.

Let $\pi_{1}: W_{1} \rightarrow \mathscr{S}_{1}$ and $\pi_{2}: W_{2} \rightarrow \mathscr{S}_{2}$ be two vector bundles and let $\left(f, f_{0}\right)$ be a vector bundle morphism so that $f: W_{1} \rightarrow W_{2}$ and $f_{0}: \mathscr{S}_{1} \rightarrow \mathscr{S}_{2}$. Then, for each $x \in \mathscr{S}_{1}$, one has the dual linear mapping $f_{x}^{*}: W_{2 f_{0}(x)}^{*} \rightarrow W_{1 x}^{*}, f_{x}^{*}\left(\phi_{2}\right)\left(w_{1}\right)=$ $\phi_{2}\left(f_{x}\left(w_{1}\right)\right)$. If $\phi: \mathscr{S}_{2} \rightarrow W_{2}^{*}$ is a section, then a section $f^{*}(\phi): \mathscr{S}_{1} \rightarrow W_{1}^{*}$ is induced by

$$
f^{*}(\phi)(x)=f_{x}^{*}\left(\phi\left(f_{0}(x)\right)\right) .
$$

The most common example for a dual bundle is the cotangent bundle $\pi: T^{*} \mathscr{S} \rightarrow$ $\mathscr{S}$ - the dual to the tangent bundle. Elements of the cotangent bundle are referred to as covectors. The local dual basis is denoted by $e^{i}=\mathrm{d} x^{i}$, so that by the definition of the dual basis

$$
\mathrm{d} x^{i}\left(\frac{\partial}{\partial x^{j}}\right)=\delta_{j}^{i} .
$$

The action of the covector $\phi=\sum_{i} \phi_{i} \mathrm{~d} x^{i}$ on the tangent vector $v=\sum_{i} v^{i} \partial / \partial x^{i}$ is computed by $\phi(v)=\sum_{i} \phi_{i} v^{i}$. Using Equation (5.I5), the transformation rules for the base vectors and for components of elements of the cotangent bundle are

$$
\mathrm{d} x^{i^{\prime}}=\sum_{i} \frac{\partial x^{i^{\prime}}}{\partial x^{i}} \mathrm{~d} x^{i}, \quad \phi_{i^{\prime}}=\sum_{i} \frac{\partial x^{i}}{\partial x^{i^{\prime}}} \phi_{i},
$$

i.e., the components of a covector transform using the transpose of the Jacobian. The equation on the left above motivates the notation $\mathrm{d} x^{i}$ for the dual base vectors. 
A section of the cotangent bundle is referred to as a 1 -form. As a special case of the definition in Equation (5.29), if $f: \mathscr{S}_{1} \rightarrow \mathscr{S}_{2}$ is a smooth mapping and $\phi: \mathscr{S}_{2} \rightarrow T^{*} \mathscr{S}_{2}$ is a I-form, then, $T f^{*}(\phi): \mathscr{S}_{1} \rightarrow T^{*} \mathscr{S}_{1}$ is the I-form over $\mathscr{S}_{1}$ induced by the dual of the tangent mapping $T_{x} f: T_{x} \mathscr{S}_{1} \rightarrow T_{f(x)} \mathscr{S}_{2}$ so that

$$
\left(T f^{*}(\phi)\right)(x)(v)=\phi(f(x))\left(T_{x} f(v)\right), \quad x \in \mathscr{S}_{1}, v \in T_{x} \mathscr{S}_{1} .
$$

The matrix representing $\left(T_{x} f\right)^{*}$ is clearly the transpose of the Jacobian matrix corresponding to the local representative of $f$. In case $\mathscr{S}_{1}$ is a submanifold of $\mathscr{S}_{2}$ and $\iota: \mathscr{S}_{1} \rightarrow \mathscr{S}_{2}$ is the inclusion embedding with $T \iota(v)=v$, then, for a I-form $\phi$ over $\mathscr{S}_{2}, T \iota^{*}(\phi)(v)=\phi(T \iota(v))=\phi(v)$ so that $T \iota^{*}(\phi)$ is simply the restriction of the form to vectors tangent to $\mathscr{S}_{1}$. We will refer to $T f^{*}(\phi)$ as the pullback of the form $\phi$ by $f$.

Example 5.8. In a general framework of kinematics and statics of a mechanical system, one considers the configuration space-a manifold $Q$ - of the system. A virtual velocity is an element of the tangent bundle $T Q$ and a generalized force is an element of the cotangent bundle $T^{*} Q$. The action of a generalized force $F \in T_{q} Q$ on a virtual velocity $v \in T_{q} Q, q \in Q$, the real number $F(v)$, is interpreted as the virtual power expended by the force on the virtual velocity. This is evidently a generalization of the expression for the virtual power in classical mechanics. This general framework may be extended to continuum mechanics, in which case the configuration space is an infinite dimensional manifold. An analysis of the structure of $T^{*} Q$ for the infinite dimensional case leads to a distinct approach to the theory of existence of stresses which is different from that presented in the following sections (see [Seg86]).

5.6. Tensor Bundles and Differential Forms. Let $\pi_{1}: W_{1} \rightarrow \mathscr{S}$ and $\pi_{2}$ : $W_{2} \rightarrow \mathscr{S}$ be two vector bundles over the same manifold $\mathscr{S}$ having typical fibers $\mathbf{V}_{1}$ and $\mathbf{V}_{2}$, respectively. Clearly, local trivializations of these vector bundles allow one to consider local trivializations that are common to both. These will induce local trivializations for vector bundles over $\mathscr{S}$ with typical fibers such as $\mathbf{V}_{1} \times \mathbf{V}_{2}$, $\mathbf{V}_{1} \otimes \mathbf{V}_{2}, L\left(\mathbf{V}_{1}, \mathbf{V}_{2}\right)$ - the vector space of linear transformations $\mathbf{V}_{1} \rightarrow \mathbf{V}_{2}$, etc. The corresponding vector bundles will be denoted respectively by $\pi_{1} \times \pi_{2}: W_{1} \times W_{2} \rightarrow$ $\mathscr{S}, \pi_{1} \otimes \pi_{2}: W_{1} \otimes W_{2} \rightarrow \mathscr{S}, L\left(\pi_{1}, \pi_{2}\right): L\left(W_{1}, W_{2}\right) \rightarrow \mathscr{S}$, etc.

In particular, such constructions may be applied to the tangent and cotangent bundles of the manifold $\mathscr{S}$. The constructions of Section 4 will produce $\pi: \bigwedge^{r}(T \mathscr{S}) \rightarrow$ $\mathscr{S}$-the bundle whose fiber at $x \in \mathscr{S}$ is $\bigwedge^{r}\left(T_{x} \mathscr{S}\right)$, the space of $r$-multivectors, and $\pi: \bigwedge^{r}\left(T^{*} \mathscr{S}\right) \rightarrow \mathscr{S}$ - the bundle whose fiber at $x \in \mathscr{S}$ is $\bigwedge^{r}\left(T_{x}^{*} \mathscr{S}\right)$, the space of $r$-alternating tensors. A section of $\pi: \bigwedge^{r}\left(T^{*} \mathscr{S}\right) \rightarrow \mathscr{S}$ is referred to as a differential $r$-form.

Let $\xi: W \rightarrow \mathscr{S}$ be a vector bundle over $\mathscr{S}$. At each $x \in \mathscr{S}$ one may consider the vector space $\bigwedge^{r}\left(T_{x} \mathscr{S}, W_{x}\right)$ of alternating $r$-linear mappings of the tangent space $T_{x} \mathscr{S}$ into $W_{x}$. Clearly, $\bigwedge^{r}\left(T_{x} \mathscr{S}, W_{x}\right)=W_{x} \otimes \bigwedge^{r}\left(T_{x}^{*} \mathscr{S}\right)$. This induces the vector bundle $\pi: \bigwedge^{r}\left(T^{*} \mathscr{S}, W\right) \rightarrow \mathscr{S}$. A section of $\bigwedge^{r}\left(T^{*} \mathscr{S}, W\right)$ will be referred to as a vector valued ( $W$-valued in this case) differential $r$-form. 
Using the properties of alternating tensors we studied in Section 4 , an $r$-form $\omega$ may be represented locally as

$$
\omega=\sum_{(\gamma)} \omega_{\gamma}\left(x^{i}\right) \mathrm{d} x^{\gamma_{1}} \wedge \cdots \wedge \mathrm{d} x^{\gamma_{r}}=\sum_{I} \frac{1}{r !} \omega_{I} \mathrm{~d} x^{i_{1}} \wedge \cdots \wedge \mathrm{d} x^{i_{r}} .
$$

If $\left\{\mathbf{g}_{1}, \ldots, \mathbf{g}_{d}\right\}$ is a basis of the typical fiber $\mathbf{W}$ of the vector bundle $W$ and $\left\{g_{1}, \ldots, g_{d}\right\}$ are the induced local base vectors for a chart in $W$, then, a $W$-valued $r$-form is represented locally by

$$
\omega(x)=\sum_{(\gamma), k} \omega_{\gamma}^{k}\left(x^{i}\right) g_{k} \otimes \mathrm{d} x^{\gamma_{1}} \wedge \cdots \wedge \mathrm{d} x^{\gamma_{r}}=\sum_{I} \frac{1}{r !} \omega_{I}^{k}\left(x^{i}\right) g_{k} \otimes \mathrm{d} x^{i_{1}} \wedge \cdots \wedge \mathrm{d} x^{i_{r}} .
$$

The operations available for alternating tensors, e.g., exterior products and contractions, apply by pointwise computations to differential forms. Thus for example, for two forms $\omega$ and $\theta$, one has the form $\omega \wedge \theta$ given by $(\omega \wedge \theta)(x)=\omega(x) \wedge \theta(x)$. For the local expressions, the base vectors $\mathrm{d} x^{1}, \ldots, \mathrm{d} x^{n}$, should be substituted in the expressions obtained in Section 4 for $e^{1}, \ldots, e^{n}$.

The transformation rules for elements of the cotangent bundle in (5.30) give the transformation rules for elements of $\bigwedge^{r}\left(T^{*} \mathscr{S}\right)$. One has

$$
\begin{aligned}
\mathrm{d} x^{\gamma_{1}^{\prime}} \wedge \cdots \wedge \mathrm{d} x^{\gamma_{r}^{\prime}} & =\sum_{I}\left(\frac{\partial x^{\gamma_{1}^{\prime}}}{\partial x^{i_{1}}} \mathrm{~d} x^{i_{1}}\right) \wedge \cdots \wedge\left(\frac{\partial x^{\gamma_{r}^{\prime}}}{\partial x^{i_{r}}} \mathrm{~d} x^{i_{r}}\right), \\
& =\sum_{I} \frac{\partial x^{\gamma_{1}^{\prime}}}{\partial x^{i_{1}}} \cdots \frac{\partial x^{\gamma_{r}^{\prime}}}{\partial x^{i_{r}}} \mathrm{~d} x^{i_{1}} \wedge \cdots \wedge \mathrm{d} x^{i_{r}} \\
& =\sum_{I,(\gamma)} \frac{\partial x^{\gamma_{1}^{\prime}}}{\partial x^{i_{1}}} \cdots \frac{\partial x^{\gamma_{r}^{\prime}}}{\partial x^{i_{r}}} \varepsilon_{\gamma_{1} \ldots \gamma_{r}}^{i_{1} \ldots i_{r}} \mathrm{~d} x^{\gamma_{1}} \wedge \cdots \wedge \mathrm{d} x^{\gamma_{r}},
\end{aligned}
$$

and using $\omega=\sum_{\left(\gamma^{\prime}\right)} \omega_{\gamma^{\prime}} \mathrm{d} x^{\gamma_{1}^{\prime}} \wedge \cdots \wedge \mathrm{d} x^{\gamma_{r}^{\prime}}=\sum_{(\gamma)} \omega_{\gamma} \mathrm{d} x^{\gamma_{1}} \wedge \cdots \wedge \mathrm{d} x^{\gamma_{r}}$,

$$
\omega_{\gamma_{1}^{\prime} \ldots \gamma_{r}^{\prime}}=\sum_{I^{\prime},(\gamma)} \varepsilon_{\gamma_{1}^{\prime} \ldots \gamma_{r}^{\prime}}^{i_{1}^{\prime} \ldots i_{r}^{\prime}} \frac{\partial x^{\gamma_{1}}}{\partial x^{i_{1}^{\prime}}} \cdots \frac{\partial x^{\gamma_{r}}}{\partial x_{r}^{i_{r}^{\prime}}} \omega_{\gamma_{1} \ldots \gamma_{r}}
$$

A particularly simple expression is obtained for the transformation rule in $\bigwedge^{n}\left(T^{*} \mathscr{S}\right)$. Since the only increasing multi-index is $\left(\gamma_{1}, \ldots, \gamma_{n}\right)=(1, \ldots, n)$,

$$
\omega_{1^{\prime} \ldots n^{\prime}}=\sum_{I} \varepsilon_{1^{\prime} \ldots n^{\prime}}^{i_{1}^{\prime} \ldots i^{\prime}} \frac{\partial x^{1}}{\partial x^{i_{1}^{\prime}}} \cdots \frac{\partial x^{n}}{\partial x_{r}^{i_{r}^{\prime}}} \omega_{1 \ldots n}=\operatorname{det}\left(\frac{\partial x^{i}}{\partial x^{i^{\prime}}}\right) \omega_{1 \ldots n} .
$$

As o-alternating tensors may be regarded as real numbers (see Section 4.8), odifferential forms are real valued functions on the manifold. In particular, for any coordinate neighborhood $U_{a} \subset \mathscr{S}$, the coordinate functions $x^{i}(x)$ induced by the chart $\varphi_{a}$ may be regarded as local o-forms. A I-alternating tensor may be identified with an element of the dual vector space so that a I-form may be identified with a section of the cotangent bundle. We will use the notation $\Omega^{r}(\mathscr{S})$ for the vector space of $r$-forms on $\mathscr{S}$, that is, sections of $\bigwedge^{r}\left(T^{*} \mathscr{S}\right)$. Similarly $\Omega^{r}(T \mathscr{S}, W)$ is the space of $W$-valued differential forms, i.e., sections of $\bigwedge^{r}\left(T^{*} \mathscr{S}, W\right)$. 
The definition of the pullback of a I-form can be naturally extended to all differential forms. Let $f: \mathscr{S}_{1} \rightarrow \mathscr{S}_{2}$ be a smooth mapping. For a o-form-a real valued function $\phi: \mathscr{S}_{2} \rightarrow \mathbb{R}-T f^{*}(\phi)(x)=\phi(f(x))$. For $0<r \leqslant n$,

$$
\left(T f^{*}(\phi)\right)(x)\left(v_{1}, \ldots, v_{r}\right)=\phi(f(x))\left(T_{x} f\left(v_{1}\right), \ldots, T_{x} f\left(v_{r}\right)\right),
$$

for all $x \in \mathscr{S}_{1}, v_{1}, \ldots, v_{r} \in T_{x} \mathscr{S}_{1}$

5.7. Jet Bundles Associated with Vector Bundles. The geometrical construction of a jet is a generalization of the notion of a derivative of a field and the jet bundle is the object where possible values of the derivatives belong.

Let $\xi: W \rightarrow \mathscr{S}$ be a vector bundle. We recall that a section $w: \mathscr{S} \rightarrow W$ of $\xi$ is represented locally in the form

$$
\left(x^{1}, \ldots, x^{n}\right) \longmapsto\left(x^{1}, \ldots, x^{n}, w^{1}\left(x^{i}\right), \ldots, w^{d}\left(x^{i}\right)\right)
$$

where a local basis $\left\{g_{1}, \ldots, g_{d}\right\}$ was used for the fibers of $W$. The tangent to the section mapping is $T w: T \mathscr{S} \rightarrow T W$ which is represented locally in the form

$$
\begin{aligned}
& \left(x^{1}, \ldots, x^{n}, v^{1}, \ldots, v^{n}\right) \longmapsto \\
& \left(x^{1}, \ldots, x^{n}, w^{1}\left(x^{i}\right), \ldots, w^{d}\left(x^{i}\right), v^{1}, \ldots, v^{n}, \sum_{i} \frac{\partial w^{1}}{\partial x^{i}} v^{i}, \ldots, \sum_{i} \frac{\partial w^{d}}{\partial x^{i}} v^{i}\right) .
\end{aligned}
$$

In a somewhat analogous way to the definition of a tangent vector, we say that two sections $w$ and $w^{\prime}$ have the same jet at $x_{0} \in \mathscr{S}$ if their tangent mappings are equal at $x_{0}$, i.e., if $T_{x_{0}} w=T_{x_{0}} w^{\prime}$. Evidently, this definition is equivalent the condition

$$
w^{k}\left(x_{0}^{i}\right)=w^{\prime k}\left(x_{0}^{i}\right), \quad \frac{\partial w^{k}}{\partial x^{j}}\left(x_{0}^{i}\right)=\frac{\partial w^{\prime k}}{\partial x^{j}}\left(x_{0}^{i}\right), \quad \text { for all } \quad k=1, \ldots, d .
$$

A collection of sections all having the same jet at $x_{0}$ is a jet at $x_{0}$. Given a section $w$, the jet it induces at $x_{0}$-the jet of $w$ at $x_{0}$-will be denoted as $j(w)\left(x_{0}\right)$. The collection of all jets of sections at $x_{0} \in \mathscr{S}$ is the jet space of the vector bundle at $x$ and is denoted as $J(W)_{x}$. Given a point $x_{0} \in \mathscr{S}$ and an element $w_{0} \in W_{x_{0}}$, the collection of all jets at $x_{0}$ such that each jet is represented by a section $w$ with $w\left(x_{0}\right)=w_{0}$ will be referred to as the jet space at $w_{0}$ and will be denoted by $J(W)_{w_{0}}$. Evidently,

$$
J(W)_{x_{0}}=\bigcup_{w_{0} \in W_{x_{0}}} J(W)_{w_{0}} .
$$

The (first) jet bundle $J(W)$ is the collection of all jets at the various points in $\mathscr{S}$ so that

$$
J(W)=\bigcup_{x \in \mathscr{S}} J(W)_{x}=\bigcup_{w \in W} J(W)_{w} .
$$

The jet bundle has two vector bundle structures

$$
\pi_{0}^{1}: J(W) \longrightarrow W, \quad \text { and } \pi^{1}: J(W) \longrightarrow \mathscr{S},
$$

where $\pi_{0}^{1}(A)=w_{0}$ if $A \in J(W)_{w_{0}}$, and $\pi^{1}(A)=x$ if $A \in J(W)_{x}$. 
Given $w_{0} \in W$, the vector space $J(W)_{w_{0}}=\left(\pi_{0}^{1}\right)^{-1}\left\{w_{0}\right\}$, may be identified with the space $L\left(T_{\xi\left(w_{0}\right)} \mathscr{S}, W_{x}\right)$ of linear mappings $T_{\xi\left(w_{0}\right)} \mathscr{S}$ to $W_{x}$. It follows that $J(W)_{w_{0}}$ is isomorphic to $L\left(\mathbb{R}^{n}, \mathbf{V}\right)$. The vector space $J(W)_{x}=\left(\pi^{1}\right)^{-1}\{x\}$ may be identified with $W_{x} \times L\left(T_{\xi\left(w_{0}\right)} \mathscr{S}, W_{x}\right)$.

Let $w: \mathscr{S} \rightarrow W$ be a section. At each point $x_{0} \in \mathscr{S}$, one has the jet $j(w)\left(x_{0}\right)$ of $w$ at $x_{0}$. Thus, $w$ induces a unique section $j(w): \mathscr{S} \rightarrow J(W), x \mapsto j(w)(x)$, of the jet bundle $\pi^{1}: J(W) \longrightarrow \mathscr{S}$. The section $j(w)$ is referred to as the (first) jet of $w$. The mapping $j$ taking a section of $\xi$ and giving a section of the jet bundle is called the jet extension mapping.

Using the local coordinates and base vectors as above, an element of $W$ is represented in the form $\left(x^{i}, u^{k}\right)=\left(x^{1}, \ldots, x^{n}, u^{1}, \ldots, u^{d}\right)$. An element of the jet bundle is therefore represented in the form $\left(x^{i}, u^{k}, A_{j}^{l}\right), i, j=1, \ldots, n$, and $k, l=1, \ldots, d$. Here, the components $A_{j}^{l}$ are possible values of derivatives of the local components of a section of $W$. In other words, if a section $w: \mathscr{S} \rightarrow W$ is represented locally as in (5.38), the jet of $w$ at $x_{0}$ is represented by $\left(x_{0}^{i}, w^{k}\left(x_{0}^{i}\right), w_{, j}^{l}\left(x_{0}^{i}\right)\right)$ where a comma indicates partial differentiation. The jet of $w, j(w): \mathscr{S} \rightarrow J(W)$, is the section represented locally by the mapping $\left(x^{i}\right) \mapsto\left(x^{i}, w^{k}\left(x^{i}\right), w_{, j}^{l}\left(x^{i}\right)\right)$, or equivalently, by the fields

$$
\left(\sum_{k} w^{k}\left(x^{i}\right) g_{k}, \sum_{k, j} w_{, j}^{k}\left(x^{i}\right) g_{k} \otimes \mathrm{d} x^{j}\right) .
$$

5.8. Integral Curves and the Flow of a Vector Field. A vector field $w: \mathscr{S} \rightarrow$ $T \mathscr{S}$ on an $n$-dimensional manifold induces a system of $n$ ordinary differential equations. We first note that a curve $c: \mathbb{R} \rightarrow \mathscr{S}$ induces a curve $\dot{c}: \mathbb{R} \rightarrow T \mathscr{S}$, by requiring that for each $t_{0} \in \mathbb{R}, \dot{c}\left(t_{0}\right)$ is the tangent vector to the curve $c$ at the point $c\left(t_{0}\right)$. Clearly, $\dot{c}$ is a lift of $c$ in the sense that $c=\tau \circ \dot{c}$, where $\tau$ is the tangent bundle projection. A curve $c$ in $\mathscr{S}$ is an integral line of the vector field $w$ if for each $t_{0} \in \mathbb{R}$, $\dot{c}\left(t_{0}\right)=w\left(c\left(t_{0}\right)\right)$. An integral line is the mathematical term corresponding to the notion of a field line in physics. If the vector field is represented locally in the form $\sum_{i} w^{i} \partial / \partial x^{i}$ and the integral line is represented locally by $x^{k}(t)$, then the tangency condition is represented locally by

$$
\frac{\mathrm{d} x^{i}}{\mathrm{~d} t}(t)=w^{i}\left(x^{k}(t)\right)
$$

a system of $n$, first order, ordinary differential equations. An initial condition for the problem is a point $x_{0} \in \mathscr{S}$ and thus the integral curve at $x_{0}$ is the integral curve $c$ such that $c(0)=x_{0}$.

It is noted that for a given smooth vector field and an initial condition $x_{0}$, the integral curve at $x_{0}$ need not necessarily be defined for all $t \in \mathbb{R}$. However, the existence theorem for ordinary differential equations implies that there is an open interval $a<t<b$ containing zero for which the solution exists and on which it is unique, i.e., any two solutions defined on that open set have to agree. The interval $(a, b)$ depends on the initial condition and may tend to zero for some sequence of initial conditions. If this is not the case, the integral lines for all points in $\mathscr{S}$ may 
be defined for all values $t \in \mathbb{R}$ and the vector field is said to be complete. Even if the vector field is not complete, for each $x_{0} \in \mathscr{S}$ there is a neighborhood $U_{x_{0}}$ containing $x_{0}$ and an interval $(a, b)$ such that the integral curves for all initial conditions $x \in U_{x_{0}}$ are defined for all $t \in(a, b)$. Let $D \subset \mathscr{S} \times \mathbb{R}$ be the collection of points $\left(x_{0}, t_{0}\right)$ such that the integral line starting at $x_{0}$ is defined for $t=t_{0}$. One can define the flow of the vector field as by

$$
\Phi: D \longrightarrow \mathscr{S}, \quad \Phi(x, t)=c(t),
$$

where $c$ is the integral curve with $c(0)=x$. Since for a smooth vector field, the solution of a differential equation depends smoothly on the initial condition, the flow is a smooth mapping. Clearly, for a complete vector field, $D=\mathscr{S} \times \mathbb{R}$. A sufficient condition for a vector field $w$ to be complete is that the support of $w$ is compact (see for example [AMR88, p. 249]). In particular, every vector field on a compact manifold without boundary, such a the sphere or the torus, is complete. Furthermore, it can be shown (e.g., [BC7O, pp. I39-I42], [War83, pp. 40-4I], [AMR88, pp. 247-248]) that if $w\left(x_{0}\right) \neq 0$, then, there is a chart in an open neighborhood of $x_{0}$ such that locally $w$ is represented by $\partial / \partial x^{1}$.

5.9. Exterior Derivatives. The exterior derivative of a differential $r$-form may be regarded as the alternation of the derivative. Unlike the case of a vector field, this operation gives invariantly an $(r+1)$-form.

We start with a zero form $\omega-$ a real valued function on the manifold. The exterior derivative $\mathrm{d} \omega$ of the zero form $\omega$ is defined to be the I-form, a covector field, given by

$$
\mathrm{d} \omega(x)(v)=\left.\frac{\mathrm{d}}{\mathrm{d} t} \omega(c(t))\right|_{t=0},
$$

where $c$ is any curve at $x$ representing the tangent vector $v$. The linear dependence of the result on $v$ and its independence of the choice of curve $c$ follows immediately from the local expression

$$
\begin{aligned}
\mathrm{d} \omega(x)(v) & =\left.\frac{\mathrm{d}}{\mathrm{d} t} \tilde{\omega}\left(x^{i}(t)\right)\right|_{t=0}, \\
& =\sum_{i} \frac{\partial \tilde{\omega}}{\partial x^{i}} \dot{x}^{i}(0),
\end{aligned}
$$

where $\tilde{\omega}$ is the local representative of the real function $\omega$. It follows from the local expression above that $\mathrm{d} \omega$ is represented locally by

$$
\sum_{i} \tilde{\omega}_{, i} \mathrm{~d} x^{i}
$$

If $\tilde{\omega}=x^{k}$ locally, then, $\mathrm{d} \omega$ is represented by $\mathrm{d} x^{k}$.

The exterior derivative of higher order forms may be defined as follows. Since, at least locally, any $r$-form may be represented as a sum of expressions such as

$$
\omega=\theta \mathrm{d} \theta^{1} \wedge \cdots \wedge \mathrm{d} \theta^{r},
$$

where $\theta, \theta^{1}, \ldots, \theta^{r}$ are real valued functions, we need to define the exterior derivative only for expressions of this form assuming it depends only on the local behavior 
of $\omega$. Thus, one sets

$$
\mathrm{d}\left(\theta \mathrm{d} \theta^{1} \wedge \cdots \wedge \mathrm{d} \theta^{r}\right)=\mathrm{d} \theta \wedge \mathrm{d} \theta^{1} \wedge \cdots \wedge \mathrm{d} \theta^{r},
$$

an $(r+1)$-form. Hence, if locally $\omega=\sum_{(\gamma)} \omega_{\gamma} \mathrm{d} x^{\gamma_{1}} \wedge \cdots \wedge \mathrm{d} x^{\gamma_{r}}$, the local expression for the exterior derivative is

$$
\mathrm{d} \omega=\sum_{(\gamma), i} \omega_{\gamma, i} \mathrm{~d} x^{i} \wedge \mathrm{d} x^{\gamma_{1}} \wedge \cdots \wedge \mathrm{d} x^{\gamma_{r}} .
$$

Comparing this expression with Example 4.2, we note that the exterior derivative may be regarded as exterior product on the left with a differential operator $\sum_{i} \partial_{i} \mathrm{~d} x^{i}$. Similarly to that example, the local expression for the exterior derivative may be written as

$$
\begin{aligned}
\mathrm{d} \omega & =\sum_{(\gamma), i}(-1)^{i-1} \omega_{\gamma_{1} \ldots \widehat{\gamma_{i}} \ldots \gamma_{r+1}, \gamma_{i}} \mathrm{~d} x^{\gamma_{1}} \wedge \cdots \wedge \mathrm{d} x^{\gamma_{r+1}} \\
& =\sum_{(\gamma), i} \omega_{\gamma_{1} \ldots \widehat{\gamma_{i}} \ldots \gamma_{r+1}, \gamma_{i}} \mathrm{~d} x^{\gamma_{i}} \wedge \mathrm{d} x^{\gamma_{1}} \wedge \cdots \wedge \widehat{\mathrm{d} x^{\gamma_{i}}} \wedge \cdots \wedge \mathrm{d} x^{\gamma_{r+1}} .
\end{aligned}
$$

Equation (5.52) implies that the exterior derivative is linear, i.e., $\mathrm{d}\left(a \omega+a^{\prime} \omega^{\prime}\right)=$ $a \mathrm{~d} \omega+a^{\prime} \mathrm{d} \omega$. In addition

$$
\mathrm{d}(\mathrm{d} \omega)=\sum_{(\gamma), i} \omega_{\gamma, i k} \mathrm{~d} x^{k} \wedge \mathrm{d} x^{i} \wedge \mathrm{d} x^{\gamma_{1}} \wedge \cdots \wedge \mathrm{d} x^{\gamma_{r}}=0
$$

since the second derivative is symmetric in the $i, k$ indices, while the exterior product is anti-symmetric. By (5.52),

$$
\mathrm{d} \omega\left(v_{1}, \ldots, v_{r+1}\right)=\sum_{(\gamma), i, K} \omega_{\gamma_{1} \ldots \gamma_{r}, i} \varepsilon_{k_{1} \ldots k_{r+1}}^{i \gamma_{1} \ldots \gamma_{r}} v_{1}^{k_{1}} \cdots v_{r+1}^{k_{r+1}} .
$$

Writing in the previous equation

$$
\varepsilon_{k_{1} \ldots k_{r+1}}^{i \gamma_{1} \ldots \gamma_{r}}=\sum_{l=1}^{r+1} \delta_{k_{l}}^{i}(-1)^{l-1} \varepsilon_{k_{1} \ldots \widehat{k}_{l} \ldots k_{r+1}}^{\gamma_{1} \ldots \gamma_{r}}
$$

one has

$$
\mathrm{d} \omega\left(v_{1}, \ldots, v_{r+1}\right)=\sum_{(\gamma), i, K, l} \omega_{\gamma_{1} \ldots \gamma_{r}, i} \delta_{k_{l}}^{i}(-1)^{l-1} \varepsilon_{k_{1} \ldots \widehat{k}_{l} \ldots k_{r+1}}^{\gamma_{1} \ldots \ldots \gamma_{r}} v_{1}^{k_{1}} \cdots v_{r+1}^{k_{r+1}},
$$

which implies

$$
\begin{aligned}
\mathrm{d} \omega\left(v_{1}, \ldots, v_{r+1}\right) & =\sum_{l, i, K}(-1)^{l-1} \omega_{k_{1} \ldots \widehat{k}_{l} \ldots k_{r+1}, i} v_{l}^{i} v_{1}^{k_{1}} \ldots \widehat{v}_{l}^{k_{l}} \ldots v_{r+1}^{k_{r+1}}, \\
& =\sum_{l, i}(-1)^{l-1} D \tilde{\omega}\left(\tilde{v}_{l}\right)\left(\tilde{v}_{1}, \ldots, \widehat{\tilde{v}}_{l}, \ldots, \tilde{v}_{r+1}\right),
\end{aligned}
$$

where in the last line the superimposed tildes, indicate local representatives, $D \tilde{\omega}$ is the derivative of the tensor field so that the directional derivative $D \tilde{\omega}\left(\tilde{v}_{l}\right)$ is regarded as an alternating $r$-tensor. 
Example 5.9. The Generalization of the Divergence Operator. Let $\omega$ be an $(n-1)$-form. Locally, $\omega$ is of the form

$$
\sum_{(\beta)} \omega_{\beta_{1} \ldots \beta_{n-1}}\left(x^{i}\right) \mathrm{d} x^{\beta_{1}} \wedge \cdots \wedge \mathrm{d} x^{\beta_{n-1}} .
$$

As in Example 4.6, $\omega$ may be represented alternatively in the form

$$
\sum_{k} \omega_{1 \ldots \widehat{k} \ldots n}\left(x^{i}\right) \mathrm{d} x^{1} \wedge \cdots \wedge \widehat{\mathrm{d} x^{k}} \wedge \cdots \wedge \mathrm{d} x^{n} .
$$

Evidently, $\omega$ has $n$ components and given a Riemannian metric, $\omega$ defines a unique vector. In the general non-Riemannian case, many physical objects that are modeled in the Riemannian case by vectors, are modeled as $(n-1)$-forms. This holds in particular for fluxes, e.g., fluxes of mass and heat, and for the electric current density. When a flux is modeled as an $(n-1)$-form, the divergence of the flux, is not a scalar as in the Riemannian situation but an $n$-form determined by a single component. The divergence operator in this setting is the exterior derivative of $\omega$ represented locally as

$$
\begin{aligned}
\sum_{k} \omega_{1 \ldots . . . . . n, k}\left(x^{i}\right) & \mathrm{d} x^{k} \wedge \mathrm{d} x^{1} \wedge \cdots \wedge \widehat{\mathrm{d} x^{k}} \wedge \cdots \wedge \mathrm{d} x^{n} \\
& =\sum_{k}(-1)^{k-1} \omega_{1 \ldots \widehat{k} \ldots n, k}\left(x^{i}\right) \mathrm{d} x^{1} \wedge \cdots \wedge \mathrm{d} x^{k} \wedge \cdots \wedge \mathrm{d} x^{n},
\end{aligned}
$$

where we used the fact that in Equation (5.53) for each $k$ there is only one term that does not vanish - the term that does not contain $\mathrm{d} x^{k}$. We conclude that the single component of $\mathrm{d} \omega$ is given by $\sum_{k}(-1)^{k-1} \omega_{1 \ldots} \ldots \hat{k}_{\ldots, n, k}$.

We show next that for an $r$-form $\omega$ and a $p$-form $\tau$,

$$
\mathrm{d}(\omega \wedge \tau)=\mathrm{d} \omega \wedge \tau+(-1)^{r} \omega \wedge \mathrm{d} \tau .
$$

Using (4.58) in (5.52) one has

$$
\begin{aligned}
& \mathrm{d}(\omega \wedge \tau) \\
= & \mathrm{d}\left(\sum_{(\alpha),(\beta)} \omega_{\alpha_{1} \ldots \alpha_{r}} \tau_{\beta_{1} \ldots \beta_{p}} \mathrm{~d} x^{\alpha_{1}} \wedge \cdots \wedge \mathrm{d} x^{\alpha_{r}} \wedge \mathrm{d} x^{\beta_{1}} \wedge \cdots \wedge \mathrm{d} x^{\beta_{p}}\right) \\
= & \sum_{(\alpha),(\beta), i} \omega_{\alpha_{1} \ldots \alpha_{r}, i} \tau_{\beta_{1} \ldots \beta_{p}} \mathrm{~d} x^{i} \wedge \mathrm{d} x^{\alpha_{1}} \wedge \cdots \wedge \mathrm{d} x^{\alpha_{r}} \wedge \mathrm{d} x^{\beta_{1}} \wedge \cdots \wedge \mathrm{d} x^{\beta_{p}} \\
& \quad+\sum_{(\alpha),(\beta), i} \omega_{\alpha_{1} \ldots \alpha_{r}} \tau_{\beta_{1} \ldots \beta_{p}, i} \mathrm{~d} x^{i} \wedge \mathrm{d} x^{\alpha_{1}} \wedge \cdots \wedge \mathrm{d} x^{\alpha_{r}} \wedge \mathrm{d} x^{\beta_{1}} \wedge \cdots \wedge \mathrm{d} x^{\beta_{p}}, \\
= & \sum_{(\alpha),(\beta), i} \omega_{\alpha_{1} \ldots \alpha_{r}, i} \tau_{\beta_{1} \ldots \beta_{p}} \mathrm{~d} x^{i} \wedge \mathrm{d} x^{\alpha_{1}} \wedge \cdots \wedge \mathrm{d} x^{\alpha_{r}} \wedge \mathrm{d} x^{\beta_{1}} \wedge \cdots \wedge \mathrm{d} x^{\beta_{p}} \\
& \quad+(-1)^{r} \sum_{(\alpha),(\beta), i} \omega_{\alpha_{1} \ldots \alpha_{r}} \tau_{\beta_{1} \ldots \beta_{p}, i} \mathrm{~d} x^{\alpha_{1}} \wedge \cdots \wedge \mathrm{d} x^{\alpha_{r}} \wedge \mathrm{d} x^{i} \wedge \mathrm{d} x^{\beta_{1}} \wedge \cdots \wedge \mathrm{d} x^{\beta_{p}},
\end{aligned}
$$


from which Equation (5.62) follows.

\section{INTEGRATION OF FORMS ON MANIFOLDS}

6.I. Partitions of Unity. A partition of unity is a technical tool that enables one to relate local and global fields.

Let $\left\{\left(\varphi_{a}, U_{a}\right)\right\}_{a=1}^{A}$ be an atlas on a manifold $\mathscr{S}$. A partition of unity on $\mathscr{S}$ subordinate to $\left\{\left(\varphi_{a}, U_{a}\right)\right\}$ is a collection $\left\{\left(u_{b}, V_{b}\right)\right\}$ of real-valued functions $u_{b}: \mathscr{S} \rightarrow \mathbb{R}$, and corresponding open covering $V_{b}$ of $\mathscr{S}, b \in B$, satisfying the following conditions for all $b$ ( $B$ need not be a finite set): a. The support, supp $u_{b}$, of $u_{b}$, that is, the closure of the collection of points for which $u_{b}$ does not vanish, is a subset of $V_{b}$ and $V_{b}$ is a subset of a coordinate neighborhood $U_{a}$ for some $a=1, \ldots, A$; b. $u_{b}(x) \geqslant 0$ for all $x \in \mathscr{S}$; c. $\sum_{b} u_{b}(x)=1$ for all $x \in \mathscr{S}$; d. each $x \in \mathscr{S}$ belongs to a finite number of sets $V_{b}$. It is noted that $\left\{\left(\left.\varphi_{a}\right|_{V_{b}}, V_{b}\right)\right\}_{b \in B}$ may serve as an atlas that may contain an infinite number of charts but each point is covered by a finite number of coordinate neighborhoods. Thus, in the sequel we will write $\left\{\left(\varphi_{b}, V_{b}, u_{b}\right)\right\}$ for the partition of unity subordinate to this atlas.

The existence of partitions of unity is a standard result of differential geometry (see [dR84, pp. 4-7], [AMR88, pp. 377-388], [Leeo2, pp. 54-55]). Partitions of unity have various applications. For example, if $\omega \in \Omega^{r}(\mathscr{S})$ is an $r$-form, then for an atlas and a subordinate partition of unity $\left\{\left(\varphi_{b}, V_{b}, u_{b}\right)\right\}$, one has $\omega=\sum_{b} \omega_{b}$, where $\omega_{b}=u_{b} \omega$, so that each of the forms $\omega_{b}$ may be represented locally using $\varphi_{b}$. Conversely, let $\left\{\omega_{b}: V_{b} \rightarrow \bigwedge^{r} V_{b}, b \in B\right\}$ be a collection of local forms. A local $\omega_{b}$ may be defined using the chart $\left(\varphi_{b}, V_{b}\right)$, and a form $\tilde{\omega}_{b}$ in $\varphi_{b}\left\{V_{b}\right\}$ so that $\omega_{b}(x)=T \varphi_{b}^{*}\left(\tilde{\omega}_{b}\right)(x)$ for $x \in V_{b}$ and $\omega_{b}(x)=0$ outside $V_{b}$. Then, $\sum_{b} u_{b} \omega_{b}$ is a smooth $r$-form on $\mathscr{S}$ determined by the local forms $\omega_{b}$ and the partition of unity. (If the local forms $\omega_{b}$ agree on the intersections of charts, it may be shown that the resulting $\omega$ does not depend on the partition of unity.

6.2. Orientation on Manifolds. The $n$-dimensional manifold $\mathscr{S}$ is orientable if there is an $n$-form $\theta$ on $\mathscr{S}$ such that $\theta(x) \neq 0$ for all $x \in \mathscr{S}$. If $\mathscr{S}$ is orientable, such an $n$-form $\theta$ that vanishes nowhere is referred to as a volume element. From the point of view of continuum mechanics, a volume element may represent a positive (or negative) density of a positive (negative, respectively) extensive property such as mass density, energy density, etc. A local representative of a volume element is of the form $\theta_{1 \ldots n}\left(x^{i}\right) \mathrm{d} x^{1} \wedge \cdots \wedge \mathrm{d} x^{n}$ for which the smooth function $\theta_{1 \ldots n}\left(x^{i}\right)$ does not vanish anywhere. We can assume that the coordinate neighborhoods that make up an atlas are connected sets for otherwise, we can restrict the charts to connected components of coordinate neighborhoods. It follows that for such an atlas, the local representatives $\theta_{1 \ldots n}\left(x^{i}\right)$ are either positive everywhere or negative everywhere. Since by reordering the coordinates we can change the sign of $\mathrm{d} x^{1} \wedge \cdots \wedge \mathrm{d} x^{n}$ we can assume that $\theta_{1 \ldots n}\left(x^{i}\right)$ are positive everywhere for every chart. It follows from Equation (5.36) that the Jacobian determinants of the coordinate transformations between all pairs of charts are positive, $\operatorname{det}\left(\partial x^{i} / \partial x^{i^{\prime}}\right)>0$. In fact, if the Jacobian determinants are positive, one can use a partition of unity to construct a volume 
element. Hence, orientability is equivalent to the existence of an atlas for which the Jacobian determinants of coordinate transformations are positive.

Given a volume element $\theta$ on an orientable manifold $\mathscr{S}$, any $n$-form $\rho$ may be represented as $\rho=u \theta$ for a real valued function $u$ defined on $\mathscr{S}$. A typical local representative $\tilde{u}$ of $u$ is given in terms of the local representatives of $\rho$ and $\theta$ by $\tilde{u}\left(x^{i}\right)=\rho_{1 \ldots n}\left(x^{i}\right) / \theta_{1 \ldots n}\left(x^{i}\right)$, where we use the fact that $\theta_{1 \ldots n}$ does not vanish.

A volume element induces an orientation on an orientable manifold $\mathscr{S}$. Two volume elements $\theta$ and $\theta^{\prime}$ induce the same orientation on $\mathscr{S}$ if $\theta^{\prime}=u \theta$ for a positive function $u$. Evidently, there may be only two orientations on a connected manifold, the one induced by $\theta$ and the one induced by $-\theta$. An orientable manifold $\mathscr{S}$ together with a choice of one of the two possible orientations is referred to as an oriented manifold. Let $\theta$ be a volume element that represents the given orientation. An atlas for which all the local representatives of $\theta$ are positive will be referred to as an oriented atlas.

An orientation of the manifold $\mathscr{S}$ induces an orientation on each of its tangent spaces. An oriented collection of $n$ linearly independent tangent vectors $\left(v_{1}, \ldots, v_{n}\right)$, $v_{i} \in T_{x} \mathscr{S}$ is positively oriented if $\theta(x)\left(v_{1}, \ldots, v_{n}\right)>0$. For a positively oriented chart, the collection of base vectors $\left(\partial / \partial x^{1}, \ldots, \partial / \partial x^{n}\right)$ is positively oriented.

We want to describe now how an orientation on a manifold with a boundary induces an orientation on the boundary. In order to do that, one has to study further the tangent spaces to the manifold at points on the boundary. Let $\mathscr{S}$ be a manifold with a boundary and $y \in \partial \mathscr{S}$. A curve $c$ at $y$ need not be defined on an interval $(-\epsilon, \epsilon)$ because such a curve is represented locally by a curve $\tilde{c}$ valued in $\mathbb{R}^{n-}$ with $\tilde{c}(0)=\left(y^{1}, \ldots, y^{n-1}, 0\right)$ and the manifold does not contain points with $y^{n}>0$. Thus, for defining the tangent space at a boundary point $y$ we admit curves represented by the restrictions to $(-\epsilon, 0]$ or to $[0, \varepsilon)$ of curves $\tilde{c}:(-\epsilon, \epsilon) \rightarrow \mathbb{R}^{n}$, for some $\epsilon>0$, with $\tilde{c}(0)=\varphi(y)=\left(y^{1}, \ldots, y^{n-1}, 0\right)$. Tangents to such curves are well defined since they are induced by the derivatives of the representing curves $\tilde{c}$ at zero. This makes the tangent space at $y$ isomorphic to $\mathbb{R}^{n}$ as expected.

A tangent vector $v \in T_{y} \mathscr{S}, y \in \partial \mathscr{S}$ is said to be inward pointing if for an oriented chart in a neighborhood of $y$ it is represented in the form $\left(v^{1}, \ldots, v^{n}\right)$ with $v^{n}<0 ; v$ is outward pointing if $v^{n}>0$. These properties do not depend on the chart chosen. Let $\iota: \partial \mathscr{S} \rightarrow \mathscr{S}$ be the natural embedding of the boundary and denote by $\tau_{\mathscr{S}}: T \mathscr{S} \rightarrow$ $\mathscr{S}$ and $\tau_{\partial \mathscr{S}}: T(\partial \mathscr{S}) \rightarrow \partial \mathscr{S}$ the tangent bundle projections for the manifold and its boundary, respectively. We also have the vector bundle $\iota^{*}\left(\tau_{\mathscr{S}}\right): \iota^{*}(T \mathscr{S}) \rightarrow \partial \mathscr{S}$ whose fiber at any point $y \in \partial \mathscr{S}$ is $T_{y} \mathscr{S}$ so that $\iota^{*}(T \mathscr{S})$ is simply the restriction of $T \mathscr{S}$ to the submanifold $\partial \mathscr{S}$.

Assuming $\mathscr{S}$ is oriented, using a partition of unity subordinate to an oriented atlas, one can construct a nowhere vanishing field of outward pointing vectors. One has to patch together local representatives of vector fields of the form $(0, \ldots, 0,1)$. The nowhere vanishing field of outward pointing vectors is a section $w: \partial \mathscr{S} \rightarrow$ $\iota^{*}(T \mathscr{S})$. Given a volume element $\theta$ on $\mathscr{S}$, a collection of linearly independent vectors $\left(v_{2}, \ldots, v_{n}\right), v_{i} \in T_{y} \partial B$ and an outward pointing vector $v$, the vectors $\left(v, v_{2}, \ldots, v_{n}\right)$ are linearly independent and so $\theta(y)\left(v, v_{2}, \ldots, v_{n}\right) \neq 0$. Thus, for any nowhere vanishing outward pointing vector field $w, w\lrcorner \theta$ is nowhere vanishing on $\partial \mathscr{S}$. It 
follows that $w\lrcorner \theta$ may serve as a volume element on $\partial \mathscr{S}$. The orientation on $\mathscr{S}$ induced by $\theta$ determines an orientation on $\partial \mathscr{S}$ for which the vectors $\left(v_{2}, \ldots, v_{n}\right)$ are positively oriented if $\left(v, v_{2}, \ldots, v_{n}\right)$ are positively oriented in $\mathscr{S}$.

Remark 6.I. There is a slight complication that stems from the definition given above for the positive orientation of the boundary. Given a coordinate neighborhood at the boundary belonging to a positively oriented atlas in $\mathscr{S}$, the form $\mathrm{d} x^{1} \wedge \cdots \wedge \mathrm{d} x^{n}$ is a positive volume element. The vector $\partial / \partial x^{n}$ is outward pointing and so the induced orientation is determined by the form represented locally as $\left.\partial / \partial x^{n}\right\lrcorner \mathrm{d} x^{1} \wedge \cdots \wedge \mathrm{d} x^{n}$ and using (4.84) one has

$$
\left.\frac{\partial}{\partial x^{n}}\right\lrcorner \mathrm{d} x^{1} \wedge \cdots \wedge \mathrm{d} x^{n}=(-1)^{n-1} \mathrm{~d} x^{1} \wedge \cdots \wedge \mathrm{d} x^{n-1} .
$$

This implies that the form $\mathrm{d} x^{1} \wedge \cdots \wedge \mathrm{d} x^{n-1}$ and the chart with coordinates $x^{1}, \ldots, x^{n-1}$ are negatively oriented if the dimension is even.

6.3. Integration on Oriented Manifolds. The natural objects to be integrated on an $n$-dimensional oriented manifold $\mathscr{S}$ are $n$-forms. An intuitive motivation for the role of differential forms as integrands is given below in Section 6.5 on integration over chains on manifolds.

Let $\rho$ be an $n$-form defined on the oriented manifold $\mathscr{S}$. Let $\left\{\left(\varphi_{b}, V_{b}, u_{b}\right)\right\}$ be a partition of unity subordinate to an oriented atlas. Expecting that the integral will be linear in the integrands, we expect that

$$
\int_{\mathscr{S}} \rho=\int_{\mathscr{S}}\left(\sum_{b} u_{b}\right) \rho=\sum_{b} \int_{\mathscr{S}} u_{b} \rho .
$$

Thus, it will be sufficient to define the integral for a form that is compactly supported on the domain of a chart. Let $\alpha$ be an $n$-form which is compactly supported in the domain of the oriented chart $\left(\varphi_{b}, V_{b}\right)$ and let the local representation of $\alpha$ be $\alpha_{1 \ldots n}\left(x^{i}\right) \mathrm{d} x^{1} \wedge \cdots \wedge \mathrm{d} x^{n}$. Then, the integral of $\alpha$ over $\mathscr{S}$ is defined by

$$
\int_{\mathscr{S}} \alpha:=\int_{\varphi_{b}\left\{V_{b}\right\}} \alpha_{1 \ldots n}\left(x^{i}\right) \mathrm{d} x^{1} \cdots \mathrm{d} x^{n} .
$$

The integral of a form on a manifold is computed now using Equation (6.2). One can show, e.g., [Leeo2, pp. 353-355], that the definition is independent of the choice of oriented atlas and partition of unity. A basic element in proving the independence of the integral on the choice of atlas is the transformation rule (5.36) for $n$-forms and its relation to the transformation rule for integrands of functions defined on $\mathbb{R}^{n}$.

It follows from the definition of the integral of a form that if one reverses the choice of orientation on the manifold $\mathscr{S}$, the opposite value will be obtained for integral of an a form $\rho$.

6.4. Stokes's Theorem. Let $\mathscr{S}$ be a compact manifold with a boundary and let $\iota$ : $\partial \mathscr{S} \rightarrow \mathscr{S}, \iota(x)=x$, be the inclusion of the boundary in $\mathscr{S}$. The inclusion mapping induces the tangent $T \iota: T(\partial \mathscr{S}) \rightarrow T \mathscr{S}, \iota(v)=v$, the inclusion of vectors tangent to the boundary in the tangent spaces to the manifold, and $T \iota^{*}: \Omega^{n-1}(\mathscr{S}) \rightarrow$ 
$\Omega^{n-1}(\partial \mathscr{S})$ that restricts $(n-1)$-forms defined on the manifold to vectors tangent to its boundary ( $c f$. the analogous Sections 4.II, 5.5, 6.2).

Consider the local representative $\sum_{k} \omega_{1 \ldots \widehat{k} \ldots n}\left(x^{i}\right) \mathrm{d} x^{1} \wedge \cdots \wedge \widehat{\mathrm{d} x^{k}} \wedge \cdots \wedge \mathrm{d} x^{n}$ of an $(n-1)$-form $\omega$ in a chart for a manifold with a boundary as in Section 5.I. As an $(n-1)$-form on an $(n-1)$-manifold, $T \iota^{*}(\omega)$ has but one component $\iota^{*}(\omega)_{1 \ldots n-1}$ given by

$$
\begin{aligned}
T \iota^{*}(\omega)_{1 \ldots n-1} & =T \iota^{*}(\omega)\left(\frac{\partial}{\partial x^{1}}, \ldots, \frac{\partial}{\partial x^{n-1}}\right) \\
& =\omega\left(T \iota\left(\frac{\partial}{\partial x^{1}}\right), \ldots, T \iota\left(\frac{\partial}{\partial x^{n-1}}\right)\right) \\
& =\omega\left(\frac{\partial}{\partial x^{1}}, \ldots, \frac{\partial}{\partial x^{n-1}}\right) \\
& =\omega_{1} \ldots n-1
\end{aligned}
$$

i.e., the component for which the omitted term $k$ equals $n$. (See Section 7.3 for a more detailed representation of $T \iota^{*}$.)

Stokes's theorem in differential geometry is a generalization of the Gauss, Green and Stokes theorems of multi-variable calculus. It does not use a Riemannian metric and it has a very compact form using integration of forms on oriented manifolds. It states that for an $(n-1)$-form $\omega$ on $\mathscr{S}$,

$$
\int_{\mathscr{S}} \mathrm{d} \omega=\int_{\partial \mathscr{S}} T \iota^{*}(\omega), \quad \text { or in short, } \quad \int_{\mathscr{S}} \mathrm{d} \omega=\int_{\partial \mathscr{S}} \omega .
$$

Using a partition of unity to localize the forms to coordinate neighborhoods, it is sufficient to prove the theorem for a compactly a supported form $\omega$ defined on an open subset $U$ of $\mathbb{R}^{n-}$, where $\partial U=U \cap \partial \mathbb{R}^{n-}$ and $\partial \mathbb{R}^{n-}=\left\{x \in \mathbb{R}^{n} \mid x^{n}=0\right\}$. Thus, we consider the integral over $\partial U$ of the $(n-1)$-form $T \iota^{*}(\omega)$, where $\omega$ is supported in a chart in a neighborhood $U$ of the boundary with oriented coordinates $x^{1}, \ldots, x^{n}$. By the definition of the integral above, we have to evaluate the integral using a positively oriented chart on $\partial U$. However, by Remark 6.I, $(-1)^{n-1} \mathrm{~d} x^{1} \wedge \cdots \wedge \mathrm{d} x^{n-1}$ is positively oriented rather than $\mathrm{d} x^{1} \wedge \cdots \wedge \mathrm{d} x^{n-1}$.

Thus,

$$
\begin{aligned}
\int_{\partial U} T \iota^{*}(\omega) & =\int_{\partial U}(-1)^{n-1} \omega_{1 \ldots n-1} \mathrm{~d} x^{1} \wedge \cdots \wedge \mathrm{d} x^{n-1} \\
& =\int_{\partial U}(-1)^{n-1} \omega_{1 \ldots n-1} \mathrm{~d} x^{1} \cdots \mathrm{d} x^{n-1}
\end{aligned}
$$

Using Example 5.9 and Equation (6.6) the local version of Equation (6.5) becomes

$$
\int_{U} \sum_{k}(-1)^{k-1} \omega_{1 \ldots \widehat{k} \ldots n, k}\left(x^{i}\right) \mathrm{d} x^{1} \cdots \mathrm{d} x^{n}=\int_{\partial U}(-1)^{n-1} \omega_{1 \ldots n-1}\left(x^{i}\right) \mathrm{d} x^{1} \cdots \mathrm{d} x^{n-1} .
$$


For the integrals on the left with $k<n$, we have

$$
\begin{aligned}
\int_{U} \omega_{1 \ldots \widehat{k} \ldots n, k} \mathrm{~d} x^{1} \cdots \mathrm{d} x^{n} & =\int_{\mathbb{R}^{n-}} \omega_{1 \ldots \widehat{k} \ldots n, k} \mathrm{~d} x^{1} \ldots \mathrm{d} x^{n}, \\
& =\int_{\mathbb{R}^{(n-1)-}}\left(\int_{\mathbb{R}} \omega_{1 \ldots \widehat{k} \ldots n, k} \mathrm{~d} x^{k}\right) \mathrm{d} x^{1} \ldots \widehat{\mathrm{d} x^{k}} \ldots \mathrm{d} x^{n},
\end{aligned}
$$

where for the integral in the parenthesis

$$
\int_{\mathbb{R}} \omega_{1 \ldots \widehat{k} \ldots n, k} \mathrm{~d} x^{k}=\left.\omega_{1 \ldots \widehat{k} \ldots . n}(x)\right|_{x^{k} \rightarrow-\infty} ^{x^{k} \rightarrow \infty}=0
$$

as $\omega$ vanishes outside a compact subset of $U$. Thus, all integrals in (6.8) vanish for $k<n$. If $U$ does not intersect $\partial \mathbb{R}^{n-}$, the same holds for $k=n$ and in this case, the right-hand side of (6.7) vanishes too. If $\partial U \neq \varnothing$, then

$$
\begin{aligned}
\int_{U} \omega_{1 \ldots n-1, n} \mathrm{~d} x^{1} \ldots \mathrm{d} x^{n} & =\int_{\mathbb{R}^{n-}} \omega_{1 \ldots n-1, n} \mathrm{~d} x^{1} \ldots \mathrm{d} x^{n}, \\
& =\int_{\mathbb{R}^{(n-1)}}\left(\int_{\mathbb{R}^{-}} \omega_{1 \ldots n-1, n} \mathrm{~d} x^{n}\right) \mathrm{d} x^{1} \ldots \mathrm{d} x^{n-1}, \\
& =\left.\int_{\mathbb{R}^{(n-1)}} \omega_{1 \ldots n-1}(x)\right|_{x^{n} \rightarrow-\infty} ^{x^{n}=0} \mathrm{~d} x^{1} \ldots \mathrm{d} x^{n-1}, \\
& =\left.\int_{\mathbb{R}^{(n-1)}} \omega_{1 \ldots n-1}(x)\right|_{x^{n}=0} \mathrm{~d} x^{1} \ldots \mathrm{d} x^{n-1} .
\end{aligned}
$$

We conclude for the left-hand side of (6.7),

$\int_{U} \sum_{k}(-1)^{k-1} \omega_{1 \ldots \widehat{k} \ldots n, k}\left(x^{i}\right) \mathrm{d} x^{1} \ldots \mathrm{d} x^{n}=\left.\int_{\mathbb{R}^{(n-1)}}(-1)^{n-1} \omega_{1 \ldots n-1}(x)\right|_{x^{n}=0} \mathrm{~d} x^{1} \ldots \mathrm{d} x^{n-1}$,

and as $\left.\omega_{1 \ldots n-1}(x)\right|_{x^{n}=0}$ vanishes outside $U$, (6.7) follows.

6.5. Integration over Chains on Manifolds. In order that geometric objects such as simplices and chains, which do not have smooth boundaries, may serve as domains of integration, integration theory is extended as described below.

The standard $r$-simplex $\Delta^{r}$ is the simplex in $\mathbb{R}^{r}$ with the first vertex at the origin such that the vectors to the other vertices are $v_{i}=\mathbf{e}_{i}, i=1, \ldots, r$, where $\mathbf{e}_{i}$ is the $i$-th standard base vector in $\mathbb{R}^{r}$. The orientation of $\Delta^{r}$ is induced by the standard orientation of $\mathbb{R}^{r}$. A singular $r$-simplex in a manifold $\mathscr{S}$ is a smooth map $s: \Delta^{r} \rightarrow \mathscr{S}$. As the simplex is not an open set, smoothness means that there is an open set $U \in$ $\mathbb{R}^{r}$ containing $\Delta^{r}$ such that $s$ is the restriction of a smooth map $s_{e}: U \rightarrow \mathscr{S}$ to $\Delta^{r}$.

We want to regard the faces of singular $r$-simplices as simplices too. So first one defines mappings $k_{p}: \Delta^{r-1} \rightarrow \mathbb{R}^{r}, p=0, \ldots, r$, and presents the faces of the standard $r$-simplex as singular $(r-1)$-simplices. These mappings are defined as

$$
k_{p}\left(x^{1}, \ldots, x^{r-1}\right)=\left(x^{1}, \ldots, x^{p-1}, 0, x^{p}, \ldots, x^{r-1}\right), \quad \text { for } \quad p=1, \ldots, r,
$$

and

$$
k_{0}\left(x^{1}, \ldots, x^{r-1}\right)=\left(1-\sum_{j=1}^{r-1} x^{j}, x^{1}, \ldots, x^{r-1}\right)
$$


The derivatives of these mappings are clearly uniform and are given in terms of the standard bases by the matrices

$$
\left[K_{p}\right]=\left[D k_{p}\right]=\left(\begin{array}{cccccc}
1 & 0 & 0 & \cdots & 0 & 0 \\
0 & \ddots & 0 & \ldots & & 0 \\
& 0 & 1 & 0 & & \\
\vdots & \vdots & 0 & 0 & \vdots & \vdots \\
\vdots & \vdots & \vdots & 1 & 0 & \\
& & & & \ddots & 0 \\
0 & 0 & 0 & \cdots & 0 & 1
\end{array}\right) \Leftarrow p \text { th row, } \quad p=1, \ldots, r
$$

and

$$
\left[K_{0}\right]=\left[D k_{0}\right]=\left(\begin{array}{rrrr}
-1 & -1 & \cdots & -1 \\
1 & 0 & \cdots & 0 \\
0 & 1 & 0 & \\
\vdots & \vdots & \ddots & \vdots \\
0 & \cdots & 0 & 1
\end{array}\right)
$$

One can verify that when applied to the standard basis $\left\{\mathbf{e}_{1}, \ldots \mathbf{e}_{r-1}\right\}$, one obtains

$$
K_{0}\left(\mathbf{e}_{l}\right)=\mathbf{e}_{l+1}-\mathbf{e}_{1}, \quad K_{p}\left(\mathbf{e}_{l}\right)=\mathbf{e}_{l}, \text { for } l<p, \quad K_{p}\left(\mathbf{e}_{l}\right)=\mathbf{e}_{l+1}, \text { for } l \geqslant p
$$

in accordance with Equation (2.22).

An $r$-singular chain on the manifold $\mathscr{S}$ is a formal linear combination $\sum_{l} a^{l} s_{l}$ of singular $r$-simplices. The chain $1 s$ is identified with $s$ and $(-1) s$ is identified with the simplex obtained from $s$ by reversing the orientation of the standard simplex. Triangulation theory implies that differentiable manifolds may be represented as chains (see [Whi57, pp. I24-I35]).

We now consider the boundary of a singular $r$-simplex $s$, the singular $(r-1)$ chain $\partial s$. In order that the definition of orientations agrees with Equation (2.22), one defines $s_{p}=s \circ k_{p}$, and

$$
\partial s=\sum_{p}(-1)^{p} s_{p}=\sum_{p}(-1)^{p} s \circ k_{p} .
$$

The boundary operator is extended to chains by linearity. It is noted that in the last equation $(-1) s$ is regarded as a multiplication of the simple chain $s$ by -1 thus reversing its orientation. It does not indicate the multiplication of the mapping $s$ by -1 even in the case where such a multiplication makes sense, e.g., $(-1)^{p} k_{p}$.

Let $s$ be a singular $r$-simplex on an $n$-dimensional manifold $\mathscr{M}$ and let $\omega$ be a smooth $r$-form defined in a neighborhood of $D=s\left(\Delta^{r}\right)$. We will refer to $\omega$ simply as a smooth form over $s$. The integral of $\omega$ over $s$ (over $D$ ) is defined by

$$
\int_{s} \omega=\int_{D} \omega=\int_{\Delta^{r}} T s^{*}(\omega)=\int_{\Delta^{r}} \omega_{1 \ldots r} \mathrm{~d} x^{1} \cdots \mathrm{d} x^{r},
$$


where, $\omega_{1 \ldots r} \mathrm{~d} x^{1} \wedge \cdots \wedge \mathrm{d} x^{r}=T s^{*}(\omega)$ is the pullback of $\omega$ to $\Delta^{r} \subset \mathbb{R}^{r}$ using the simplex mapping $s$. The definition of the integral implies that if one reverses the choice of orientation on the simplex $s$, the opposite value will be obtained for the integral of a form $\omega$.

The definition of the integral of a form over a simplex suggests an intuitive motivation for the role of differential forms as integrands. For a singular simplex $s$, and using the mean value theorem for integration in $\mathbb{R}^{r}$, there is a point $P \in \Delta^{r}$ such that

$$
\begin{aligned}
\int_{s} \omega & =\int_{\Delta^{r}} \omega_{1 \ldots r} \mathrm{~d} x^{1} \cdots \mathrm{d} x^{r} \\
& =\omega_{1 \ldots r}(P) \int_{\Delta^{r}} \mathrm{~d} x^{1} \cdots \mathrm{d} x^{r}, \\
& =\frac{1}{r !} \omega_{1 \ldots r}(P) .
\end{aligned}
$$

Letting $\left\{\mathbf{e}_{1}, \ldots, \mathbf{e}_{r}\right\}$ be the standard basis of $\mathbb{R}^{r}$ and $v_{i}=T s(P)\left(\mathbf{e}_{i}\right)$,

$$
\begin{aligned}
\omega_{1 \ldots r}(P) & =T s^{*}(\omega)(P)\left(\mathbf{e}_{1}, \ldots, \mathbf{e}_{r}\right), \\
& =\omega(s(P))\left(v_{1}, \ldots, v_{r}\right) .
\end{aligned}
$$

We conclude that

$$
\int_{s} \omega=\frac{1}{r !} \omega(s(P))\left(v_{1}, \ldots, v_{r}\right)
$$

so that the integral is approximated by the evaluation of the form on the vectors tangent to the edges of $D$.

Remark 6.2. The last equation contradicts our interpretation of the action of an alternating tensor on the $r$-tuple of vectors $\left(v_{1}, \ldots, v_{r}\right)$ as providing the total flux through the oriented simplex they determine. The division by $r$ ! implies that for an alternating tensor $T, T\left(v_{1}, \ldots, v_{r}\right)$ should be interpreted as the flux corresponding to the $r$-cube that the vectors determine. This inconsistency follows from the definition of the integral as in Equations (6.3) and (6.18). The interpretation of the action as a flux through the simplex would be accurate if the integral would be defined alternatively as

$$
r ! \int_{\Delta^{r}} \omega_{1 \ldots r} \mathrm{~d} x^{1} \cdots \mathrm{d} x^{r}=\int_{\Delta^{r}} \sum_{\pi}(-1)^{|\pi|} \omega_{\pi_{1} \ldots \pi_{r}} \mathrm{~d} x^{\pi_{1}} \cdots \mathrm{d} x^{\pi_{r}} .
$$

Nevertheless, since we rely on the simplex constructions in Section 3, since we do not want to change the traditional definition of the integral of a form, and since for simplicity of notation we do not want to write the flux through the simplex as $T\left(v_{1}, \ldots, v_{r}\right) / r$ !, we ignore this inconsistency.

Let $\pi: \mathscr{N} \rightarrow \mathscr{M}$ be a fiber bundle and $s: \Delta^{r} \rightarrow \mathscr{M}$ a singular simplex. We say that $w$ is a smooth section of $\mathscr{N}$ over $s$ if it is the restriction of a smooth section of $\mathscr{N}$ to Image $s$. If $B=\sum_{l} a^{l} s_{l}$ is an $r$-chain on $\mathscr{M}$, a smooth section $w$ of $\mathscr{N}$ over $B$ is a collection of smooth sections over the various simplices that make up $B$. (Thus, $w$ is not necessarily extendable to a global smooth section.) 
If $B=\sum_{l} a^{l} s_{l}$ is an $r$-chain, the integral of a smooth form $\omega$ over $B$ is defined by linearity as

$$
\int_{B} \omega=\sum_{l} a^{l} \int_{D_{l}} \omega_{l}=\sum_{l} a^{l} \int_{s_{l}} \omega_{l}, \quad D_{l}=s_{l}\left\{\Delta^{r}\right\},
$$

where $\omega_{l}$ is the smooth form over $s_{l}$ in the definition of a smooth section over a chain.

By subdividing an $r$-chain into small simplices $s_{l}$ and letting $Q_{l} \in D_{l}=s_{l}\left(\Delta_{r}\right)$, the integral of a form over a chain may be approximated by a sum in the form

$$
\frac{1}{r !} \sum_{l} a^{l} \omega\left(Q_{l}\right)\left(v_{1 l}, \ldots, v_{r l}\right),
$$

where $v_{p l}$ is the tangent to the $p$-th edge of $D_{l}$.

Using an analogous procedure to the proof of the Stokes theorem for oriented manifolds, in particular, the fundamental theorem of calculus, Stokes's theorem for chains on manifolds may be proved in the form (see for example [War83, AMR88, LeeO2])

$$
\int_{B} \mathrm{~d} \omega=\int_{\partial B} \omega
$$

for any $r$-chain $B$.

Remark 6.3. It is noted that in addition to allowing non-smooth boundaries and enabling integration of forms on regions of dimension lower than $\operatorname{dim} \mathscr{S}$, integration theory over chains does not require that the manifold be orientable. Furthermore, the term "singular" is used in order to indicate that in general there is no requirement that $s$ will be injective. However, integration on chains was presented above as a tool that allows one to consider the non-smooth boundaries of regular (rather than singular) simplices on manifolds. Other approaches that allow that are available (e.g., the standard manifolds of [Whis7, pp. IO8-IIO], or manifolds with corners as in [Leeo2, pp. 363-368]). Integration theory on chains seems to us to have an appealing geometric flavor. In the sequel, for all chains on manifolds that we will consider, the mappings $s_{e}$ will be non-singular so that their inverses may serve as charts.

\section{Balance Principles and Fluxes}

One of the basic notions of continuum mechanics is that of an extensive property. The term extensive property is used to describe a property that may be assigned to subsets of a given universe. This includes for example, the mass of the various parts of a material body, the electrical charge enclosed in regions in space, etc. Thus, an extensive property is a real valued set function $p$. These notes consider extensive properties that may be represented by smooth densities. For less regular extensive properties see, for example, [Sil85, GWZ86, NV88, DMM99, RSo3]. 
7.I. Densities of Extensive Properties. The setting of the theory of extensive properties presented here considers a fixed physical space modeled by an $n$-dimensional differentiable manifold $\mathscr{S}$. Alternatively, one may wish to interpret $\mathscr{S}$ as the material manifold so a point $x \in \mathscr{S}$ is a material point having an invariant meaning. Since we are going to use integration later on, we will assume that $\mathscr{S}$ is orientable and that a particular orientation was chosen.

We will refer to "regions" for which we can define the total amount of the property $p$ as control regions when we interpret $\mathscr{S}$ as the space manifold and as subbodies when we interpret $\mathscr{S}$ as the material manifold. The term "region" will be used when the particular interpretation is immaterial. Thus, admissible regions are compact $n$-dimensional submanifolds with boundary of $\mathscr{S}$ or chains in $\mathscr{S}$. In accordance with the smooth setting, it is assumed that there is an $n$-form $\rho$ defined on $\mathscr{S}$ that models the density of the property $p$. Using integration of forms, one can now calculate the total amount of the property

$$
p(\mathscr{R})=\int_{\mathscr{R}} \rho
$$

in any region $\mathscr{R}$ for which the integral is defined.

From a naive point of view, spacetime, or the event space, has the structure of a Cartesian product $\mathscr{S} \times \mathbb{R}$ so that any event may be assigned a specific location in $\mathscr{S}$ and a specific time $t \in \mathbb{R}$. Our ability to assign a particular pair of time and place to any event implies that we have a particular global frame on spacetime ( $c f$. Example 5.5). A more general structure of spacetime will be considered later in this section. This means that in general, the density $\rho$ of a property $p$ should be time dependent. Since the value $\rho(x, t) \in \bigwedge^{n}\left(T_{x}^{*} \mathscr{S}\right)$ - a fixed vector space, we may differentiate it with respect to the time variable and obtain the $n$-form $\beta=\partial \rho / \partial t$ on $\mathscr{S}$. Thus, for a fixed region $\mathscr{R}$

$$
\frac{d p(\mathscr{R})}{d t}=\int_{\mathscr{R}} \beta
$$

represents the rate of change of the amount of the property $p$ inside $\mathscr{R}$.

In the classical setting of continuum mechanics it is assumed that the change of the amount of property within the region $\mathscr{R}$ is a result of two phenomena: the rate at which the property is produced inside $\mathscr{R}$ and the rate at which the property leaves $\mathscr{R}$ through its boundaries - the flux of $p$.

The flux of the property is assumed to be distributed smoothly on the boundary of $\mathscr{R}$. Hence, whether the admissible regions are compact $n$-dimensional submanifolds of $\mathscr{S}$ or chains, integration of $(n-1)$-forms on their boundaries is well defined. It is assumed that for each region $\mathscr{R}$, there is an $(n-1)$-form $\tau_{\mathscr{R}}$ called the flux density such that the flux of $p$ is given as

$$
\int_{\partial \mathscr{R}} \tau \mathscr{R} .
$$

In the sequel when no confusion can occur, we will omit the $\mathscr{R}$ subscript and use only $\tau$. 
The production rate of the property inside $\mathscr{R}$ is assumed to be represented as

$$
\int_{\mathscr{R}} \varsigma
$$

where, $\varsigma$ is an $n$-form on $\mathscr{S}$, the source density, which is independent of $\mathscr{R}$. Thus, the classical balance law assumes the form

$$
\int_{\mathscr{R}} \beta=\int_{\mathscr{R}} \varsigma-\int_{\partial \mathscr{R}} \tau_{\mathscr{R}}
$$

for each admissible region $\mathscr{R}$.

Equation (7.3) implies that

$$
\begin{aligned}
\left|\int_{\partial \mathscr{R}} \tau_{\mathscr{R}}\right| & =\left|\int_{\mathscr{R}}(\varsigma-\beta),\right| \\
& \leqslant \int_{\mathscr{R}}|\varsigma-\beta|,
\end{aligned}
$$

where the absolute value of an $n$-form is defined as follows. Let $\theta$ be a volume element on $\mathscr{S}$ which is compatible with its orientation and let $\omega$ be an $n$-form. Then, since the space $\bigwedge^{n}\left(T_{x}^{*} \mathscr{S}\right)$ is one-dimensional for each $x \in \mathscr{S}, \omega(x)=a(x) \theta(x)$. The sign of $\omega(x)$ is identified with the sign of $a(x)$ and $|\omega|(x)=|a(x)| \theta(x)$.

For various results we present later, in particular Cauchy's theorem on the existence of flux forms, the balance principle (7.3) is regarded in view of the inequality above as a boundedness or regularity postulate on the fluxes for the various bodies. The boundedness postulate for the fluxes states that there is a bounded nonnegative $n$-form $\varsigma_{0}$ on $\mathscr{S}$ such that for any region $\mathscr{R}$

$$
\left|\int_{\partial \mathscr{R}} \tau_{\mathscr{R}}\right| \leqslant \int_{\mathscr{R}} \varsigma_{0} .
$$

On the one hand, this form of the balance principle has the essential ingredients needed later for the proof of Cauchy's theorem and on the other hand, it does not require the specification of both $\beta$ and $\varsigma$.

7.2. Flux forms and Cauchy's formula. While the rate of change of the property and the production term are specified by fields defined on the manifold $\mathscr{S}$, the flux term is specified by means of a system of forces - a set-function $\mathscr{R} \mapsto \tau_{\mathscr{R}}$ whose domain is the collection of all admissible regions. Thus, it is noted that for a fixed point $x \in \mathscr{S}$ that is on the boundary of two distinct smooth submanifolds $\mathscr{R}$ and $\mathscr{R}^{\prime}$ the values of the fluxes densities $\tau_{\mathscr{R}}(x) \in \bigwedge^{n-1}\left(T_{x}^{*} \mathscr{R}\right)$ and $\tau_{\mathscr{R}^{\prime}}(x) \in \bigwedge^{n-1}\left(T_{x}^{*} \mathscr{R}^{\prime}\right)$ belong to different spaces and cannot be compared.

Nevertheless, the integration theories presented above provide a simple means for specifying the flux densities for the admissible regions. Let $J$ be an $(n-1)$-form on $\mathscr{S}$. For every region $\mathscr{R}$, the inclusion $\iota_{\partial \mathscr{R}}: \partial \mathscr{R} \rightarrow \mathscr{S}$ induces the restriction $\rho_{\partial \mathscr{R}}(J)=T \iota_{\partial \mathscr{R}}^{*}(J), \rho_{\partial \mathscr{R}}=T \iota_{\partial \mathscr{R}}^{*}: \Omega^{n-1}(\mathscr{S}) \rightarrow \Omega^{n-1}(\partial \mathscr{R})$, as in Sections 4 .II and 6.4. We will refer to such an $(n-1)$-form $J$ as a flux form. 
Thus, a flux form induces a system of flux densities for the boundaries of the various regions by

$$
\tau_{\mathscr{R}}=T \iota_{\partial \mathscr{R}}^{*}(J)=\rho_{\partial \mathscr{R}}(J) .
$$

The last equation will be referred to as the generalized Cauchy formula and we will often omit the $\partial \mathscr{R}$-index if the particular region under consideration is clear from the context. The definition of the restriction of forms implies that for a point $x_{0} \in \mathscr{S}$ and any region $\mathscr{R}$ such that $x_{0} \in \partial \mathscr{R}$, we have for any collection $v_{1}, \ldots, v_{n-1}$ of vectors in $T_{x_{0}} \partial \mathscr{R}$,

$$
\begin{aligned}
\tau_{\mathscr{R}}\left(v_{1}, \ldots, v_{n-1}\right) & =J\left(T \iota\left(v_{1}\right), \ldots, T \iota\left(v_{n-1}\right)\right), \\
& =J\left(v_{1}, \ldots, v_{n-1}\right) \\
& =J\left(v_{1} \wedge \cdots \wedge v_{n-1}\right)
\end{aligned}
$$

where in the last line we emphasize the point of view that the flux form is applied to the multi-vector representing the infinitesimal oriented simplex defined by the vectors. The relation between the current presentation of flux theory in terms of a flux form and the traditional formulation in terms of a flux vector field was discussed in Example 4.4.

It is one of the main results of continuum mechanics, namely Cauchy's theorem, that under rather general assumptions, referred to as Cauchy's postulates, a system of flux densities is induced by a unique flux form using Cauchy's formula. Presenting a version of Cauchy's formula and theorem for the general situation where a Riemannian metric is not available is one of the main objectives of these notes.

7.3. Extensive Properties and Cauchy Formula-Local Representation. We now present the coordinate description of the objects and relations given above. Let $x^{1}, \ldots, x^{n}$ be a coordinate system in a neighborhood of a point $x_{0}$. Then, omitting the dependence on time, the $n$-forms $\rho$ and $\beta$ are represented locally using the scalar functions $\rho_{1 \ldots n}\left(x^{i}\right)$ and $\beta_{1 \ldots n}\left(x^{i}\right)$ as $\rho(x)=\rho_{1 \ldots n}\left(x^{i}\right) d x^{1} \wedge \cdots \wedge d x^{n}$ and $\beta(x)=\beta_{1 \ldots n}\left(x^{i}\right) d x^{1} \wedge \cdots \wedge d x^{n}$, respectively.

The flux density $\tau_{\mathscr{R}}$ should be represented using a coordinate system on the $(n-1)$-dimensional manifold $\partial \mathscr{R}$, say $y^{1}, \ldots, y^{n-1}$. Thus, in such a coordinate system, $\tau_{\mathscr{R}}$ is represented in a neighborhood of the boundary point $y_{0}$ using the scalar function $\tau_{\mathscr{R} 1 \ldots n-1}$ in the form

$$
\tau_{\mathscr{R}}(y)=\tau_{\mathscr{R} 1 \ldots n-1}\left(y^{j}\right) d y^{1} \wedge \cdots \wedge d y^{n-1} .
$$

The local expression for the flux form $J \in \Omega^{n-1}(\mathscr{S})$, in a coordinate neighborhood $x^{1}, \ldots, x^{n}$, is (cf. Example 4.4)

$J(x)=\sum_{(\alpha)} J_{\alpha_{1} \ldots \alpha_{n-1}}(x) \mathrm{d} x^{\alpha_{1}} \wedge \cdots \wedge \mathrm{d} x^{\alpha_{n-1}}=\sum_{k} J_{1 \ldots \widehat{k} \ldots n}(x) \mathrm{d} x^{1} \wedge \cdots \wedge \widehat{\mathrm{d} x^{k}} \wedge \cdots \wedge \mathrm{d} x^{n}$

and

$$
J_{\alpha_{1} \ldots \alpha_{n-1}}=J\left(\frac{\partial}{\partial x^{\alpha_{1}}}, \ldots, \frac{\partial}{\partial x^{\alpha_{n-1}}}\right)
$$


Locally, the inclusion $\partial \mathscr{R} \rightarrow \mathscr{S}$ is represented by $n$ functions $x^{i}=x^{i}\left(y^{1}, \ldots, y^{n-1}\right)$. Thus we have

$$
T \iota\left(\frac{\partial}{\partial y^{p}}\right)=\sum_{i} x_{, p}^{i} \frac{\partial}{\partial x^{i}}, \quad p=1, \ldots, n-1,
$$

and for a vector $v \in T_{x_{0}} \partial \mathscr{R}$ represented locally by $v=\sum_{p} v^{p} \partial / \partial y^{p}$, we have $T \iota(v)=\sum_{p, i} x_{, p}^{i} v^{p} \partial / \partial x^{i}$ which we may write with some abuse of notation as $v^{i}=\sum_{p} x_{, p}^{i} v^{p}$.

The evaluation $\tau_{\mathscr{R}}\left(v_{1}, \ldots, v_{n-1}\right)$ is represented as

$$
\begin{aligned}
\tau_{\mathscr{R}}( & \left.v_{1}, \ldots, v_{n-1}\right) \\
& =\tau_{\mathscr{R} 1 \ldots n-1} d y^{1} \wedge \cdots \wedge d y^{n-1}\left(\sum_{\rho_{1}} v_{1}^{\rho_{1}} \frac{\partial}{\partial y^{\rho_{1}}}, \ldots, \sum_{\rho_{n-1}} v_{n-1}^{\rho_{n-1}} \frac{\partial}{\partial y^{\rho_{n-1}}}\right), \\
& =\sum_{\rho} \tau_{\mathscr{R} 1 \ldots n-1} v_{1}^{\rho_{1}} \cdots v_{n-1}^{\rho_{n-1}} d y^{1} \wedge \cdots \wedge d y^{n-1}\left(\frac{\partial}{\partial y^{\rho_{1}}}, \ldots, \frac{\partial}{\partial y^{\rho_{n-1}}}\right), \\
& =\sum_{\rho} \tau_{\mathscr{R} 1 \ldots n-1} v_{1}^{\rho_{1}} \cdots v_{n-1}^{\rho_{n-1}} \varepsilon_{\rho_{1} \ldots \rho_{n-1}}, \\
& =\tau_{\mathscr{R} 1 \ldots n-1} \operatorname{det}\left[\left(v_{p}\right)^{q}\right], \quad p, q, \rho_{p}=1, \ldots, n-1 .
\end{aligned}
$$

The evaluation of the flux form $J$ is represented locally as in Equation (4.74) by

$$
\begin{aligned}
\tau_{\mathscr{R}}\left(v_{1}, \ldots, v_{n-1}\right)=J & \left(v_{1}, \ldots, v_{n-1}\right) \\
& =\operatorname{det}\left(\left[(-1)^{k-1} J_{1} \ldots \widehat{k}_{\ldots n}\right] ; v_{1}^{\alpha_{1}} ; \ldots \ldots ; v_{n-1}^{\alpha_{r-1}}\right),
\end{aligned}
$$

where, $i, \alpha_{i}=1, \ldots, n-1$. We use the local representations of Cauchy's formula (7.7) to obtain

$$
\begin{aligned}
\tau_{\mathscr{R} 1 \ldots n-1} & =\tau\left(\frac{\partial}{\partial y^{1}}, \ldots, \frac{\partial}{\partial y^{n-1}}\right), \\
& =J\left(T \iota\left(\frac{\partial}{\partial y^{1}}\right), \ldots, T \iota\left(\frac{\partial}{\partial y^{n-1}}\right)\right), \\
& =J\left(\sum_{\alpha_{1}} x_{, 1}^{\alpha_{1}} \frac{\partial}{\partial x^{\alpha_{1}}}, \ldots, x_{, n-1}^{\alpha_{n-1}} \frac{\partial}{\partial x^{\alpha_{n-1}}}\right), \\
& =\operatorname{det}\left(\left[(-1)^{k-1} J_{1 \ldots \widehat{k} \ldots n}\right] ; x_{, 1}^{\alpha_{1}} ; \ldots \ldots ; x_{, n-1}^{\alpha_{n-1}}\right) .
\end{aligned}
$$

Thus, the density of the flux through the boundary is obtained by the determinant of the matrix comprised of the components of the flux form in the first column and the matrix of the derivatives of $x^{i}=x^{i}\left(y^{p}\right)$ representing the embedding of the boundary $\partial \mathscr{R}$ in $\mathscr{R}$.

7.4. Cauchy's Flux Theorem. We now present sufficient conditions for the existence of flux forms, i.e., conditions for Cauchy's formula to hold for some $(n-1)$ form $J$ over $\mathscr{S}$. Clearly, if such a form exists, it is unique. The assumptions made so far may be summarized as follows. 
Assumption 1: Boundedness. For any admissible region $\mathscr{R}$ there is a smooth $(n-1)$-form $\tau_{\mathscr{R}}$ such that the bound (7.5) holds for some non-negative bounded $n$-form $\varsigma$ (we omit the subscript o for simplicity) defined on $\mathscr{S}$.

The next assumption traditionally known as Cauchy's postulate is a locality assumption. Before making it, we note that for a point $x \in \mathscr{S}$, the evaluation $\tau_{\mathscr{R}}(x)\left(v_{1}, \ldots, v_{n-1}\right)$ is well-defined only if $v_{1}, \ldots, v_{n-1} \in T_{x} \partial \mathscr{R}$. We interpret $\tau_{\mathscr{R}}(x)\left(v_{1}, \ldots, v_{n-1}\right)$ as the infinitesimal flux of the property out of the region $\mathscr{R}$ through the infinitesimal oriented simplex induced by the positively oriented collection $v_{1}, \ldots, v_{n-1}$. In case the collection of vectors is negatively oriented, $\tau_{\mathscr{R}}(x)\left(v_{1}, \ldots, v_{n-1}\right)$ is interpreted as the infinitesimal flux into the region.

Assumption 2: Cauchy's Postulate of Locality. Let $v_{1}, \ldots, v_{n-1} \in T_{x} \mathscr{S}, x \in \mathscr{S}$, be a collection of $n-1$ vectors. Let $\mathscr{R}$ be any region such that $x \in \partial \mathscr{R}, v_{1}, \ldots, v_{n-1} \in T_{x} \partial \mathscr{R}$, and the collection of vectors is positively oriented relative to the orientation of $\partial \mathscr{R}$. Then, $\tau_{\mathscr{R}}(x)\left(v_{1}, \ldots, v_{n-1}\right)$ is independent of $\mathscr{R}$.

Cauchy's postulate implies that for the given point $x \in \mathscr{S}, \tau_{\mathscr{R}}(x)$ depends on $\mathscr{R}$ only through the tangent space $T_{x} \partial \mathscr{R}$ and the orientation induced on it. In other words, let $\iota_{\partial \mathscr{R}}: \partial \mathscr{R} \rightarrow \mathscr{S}$ be the natural embedding, and let $T \iota_{\partial \mathscr{R}}: T(\partial \mathscr{R}) \rightarrow T \mathscr{S}$ be its tangent mapping. It follows that if for two regions $\mathscr{R}$ and $\mathscr{R}^{\prime}, T \iota_{\partial \mathscr{R}}\left\{T_{x} \partial \mathscr{R}\right\}=$ $T \iota_{\partial \mathscr{R}}\left\{T_{x} \partial \mathscr{R}^{\prime}\right\}$ and the orientations induced on $T \iota_{\partial \mathscr{R}}\left\{T_{x} \partial \mathscr{R}\right\}$ and $T \iota_{\partial \mathscr{R}^{\prime}}\left\{T_{x} \partial \mathscr{R}^{\prime}\right\}$ are identical, then, $\tau_{\mathscr{R}}(x)=\tau_{\mathscr{R}}(x)$. Cauchy's postulate makes it possible to define a mapping

$$
\mathfrak{t}_{x}:\left(T_{x} \mathscr{S}\right)^{n-1} \longrightarrow \mathbb{R}
$$

such that for any collection $v_{1}, \ldots, v_{n-1}$ of vectors

$$
\mathfrak{t}_{x}\left(v_{1}, \ldots, v_{n-1}\right)=\tau_{\mathscr{R}}(x)\left(v_{1}, \ldots, v_{n-1}\right)
$$

for any region $\mathscr{R}$ such that the collection $v_{1}, \ldots, v_{n-1} \in T_{x} \partial \mathscr{R}$ are positively oriented. Thus, the order in which the collection is written, specifies the orientation for which $\mathfrak{t}_{x}\left(v_{1}, \ldots, v_{n-1}\right)$ applies. We observe that the mapping $\mathfrak{t}_{x}$ is analogous to the mapping $T$ for homogeneous fluxes as in Equation (3.16). At this point, for an odd permutation $\pi$, there is no relation between $\mathfrak{t}_{x}\left(v_{1}, \ldots, v_{n-1}\right)$ and $\mathfrak{t}_{x}\left(v_{\pi_{1}}, \ldots, v_{\pi_{n-1}}\right)$. It is noted that because of the compatibility with $\tau_{\mathscr{R}}, \mathfrak{t}_{x}\left(v_{1}, \ldots, v_{n-1}\right)$ depends only on the multi-vector $\mathfrak{v}=v_{1} \wedge \cdots \wedge v_{n-1}$. In other words, $\mathfrak{t}_{x}\left(v_{1}, \ldots, v_{n-1}\right)$ depends on the arguments multi-linearly as long as the arguments are restricted to some arbitrary $(n-1)$-dimensional hyperplane. Globally, we have a mapping,

$$
\mathfrak{t}: T^{n-1} \mathscr{S} \longrightarrow \mathbb{R}, \quad \text { such that } \mathfrak{t}_{\left(T_{x} \mathscr{S}\right)^{n-1}}=\mathfrak{t}_{x},
$$

where $T^{n-1} \mathscr{S} \rightarrow \mathscr{S}$ is the vector bundle whose fiber at $x \in \mathscr{S}$ is $\left(T_{x} \mathscr{S}\right)^{n-1}$. We will refer to $t$ as the Cauchy-Whitney map. Since $T^{n-1} \mathscr{S}$ is a vector bundle over $\mathscr{S}$-a differentiable manifold, the next assumption may be made. 
Assumption 3: Regularity. The mapping $t$ is smooth.

We will refer to the collection $\left\{\tau_{\mathscr{R}}\right\}$ for the various regions $\mathscr{R}$ satisfying the foregoing assumptions as a system of Cauchy fluxes. To prove the existence of an $n$-from $J$, such that $\tau_{\mathscr{R}}(x)=T_{x} \iota_{\partial \mathscr{R}}^{*}(J(x))$, we show that the assumptions of Section 3.I are satisfied so that the algebraic Cauchy theorem as in Section 3.4, may be used.

We first note that by its definition in terms of $\tau_{\mathscr{R}}$, the mapping $\mathfrak{t}_{x}$ satisfies the additivity assumption (3.4). The homogeneity assumption in Equation (3.2) also follows from the homogeneity of $\tau_{\mathscr{R}}$. It remains to show that the balance assumption of Section 3.I holds. As mentioned in Remark 3.2, once balance holds, the additivity assumption of Equation (3.5) is satisfied.

7.4.2. Notation. Let $v_{1}, \ldots, v_{n} \in T_{x} \mathscr{S}$ be an oriented collection of vectors. We use $S$ to denote the simplex $\left[v_{1}, \ldots, v_{n}\right]$ and let $S_{p}, p=0, \ldots, n$, be the oriented faces that make up its boundary, i.e., $\partial S=\sum_{p} S_{p}$. In view of (2.22) $S_{0}=\left[v_{2}-v_{1}, \ldots, v_{n}-v_{1}\right]$, and $S_{p}=(-1)^{p}\left[v_{1}, \ldots, \widehat{v}_{p}, \ldots, v_{n}\right], p>0$. Since the Cauchy mapping $\mathfrak{t}_{x}$ applies only to positively oriented collections of vectors, we will represent each $S_{p}, p>0$, in the form

$$
S_{p}=\left[v_{\pi_{1}^{p}}^{p}, \ldots, \widehat{v}_{p}, \ldots, v_{\pi_{n-1}^{p}}\right],
$$

where each $\pi^{p}, p=1, \ldots, n$ is a particular permutation of $n-1$ symbols chosen such that $(-1)^{\left|\pi^{p}\right|}=(-1)^{p}$. (For an even value of $p, \pi^{p}$ will be taken as the identity.) Consider the increasing injection $v^{p}:\{1, \ldots, n-1\} \rightarrow\{1, \ldots, n\}$, $p \notin$ Image $v^{p}$, and let $\mu^{p}=v^{p} \circ \pi^{p}$, then each face may be represented in the form $S_{p}=\left[v_{\mu_{1}^{p}}, \ldots, v_{\mu_{n-1}^{p}}\right], p>0$. The $l$-th vector defining $S_{p}$ in this way will be denoted by $S_{p l}$, i.e., $S_{p l}=v_{\mu_{l}^{p}}$ for $p>0$. We will extend the notation to $S_{0}$ so that $S_{0 l}=v_{l+1}-v_{1}$. Thus, $S_{p l}$ are well defined for all $p=0, \ldots, n$ and $l=1, \ldots, n-1$. Evidently, the simplices $S$ and $S_{p}$ are viewed as simplices in an affine space as in Sections 2 and 3. We have to show that

$$
\mathfrak{t}_{x}(\partial S)=\sum_{p=0}^{n} \mathfrak{t}_{x}\left(S_{p}\right)=0 .
$$

Consider a chart $(\varphi, U)$ in a neighborhood of $x$. Since we can always adjust the chart mapping by a composition of a linear mapping and a translation, we may assume without loss of generality that $\varphi(x)=0$ and $T \varphi\left(v_{i}\right)=\mathbf{e}_{i},\left\{\mathbf{e}_{i}\right\}$ being the standard basis of $\mathbb{R}^{n}$.

We also want to exhibit the oriented faces of the standard simplex as mappings defining oriented singular simplices (as opposed to the faces $k_{p}$ defined in Section 6.5 that do not specify the correct orientation). We define the maps $\kappa_{p}: \Delta^{n-1} \rightarrow$ $\mathbb{R}^{n}$ by

$$
\kappa_{p}\left(x^{1}, \ldots, x^{n-1}\right)=k_{p}\left(x^{\pi_{1}^{p}}, \ldots, x^{\pi_{n-1}^{p}}\right)
$$

where $k_{p}$ are the faces of the standard simplex defined in $(6.12,6.13)$. For an odd value of $p, D \kappa_{p}$ is obtained from $D k_{p}$ by switching columns of the matrices. Similarly to the notation $S_{p l}$, we will use $\mathfrak{e}_{p l}$ to denote the $l$-th vector in the $p$-th oriented face of $\Delta^{n-1}$. It follows that

$$
\mathfrak{e}_{p l}=T_{x} \varphi\left(S_{p l}\right)=D \kappa_{p}\left(\mathbf{e}_{l}\right), \quad p=0, \ldots, n, \quad l=1, \ldots, n-1 .
$$


As $V=\varphi(U)$ is an open neighborhood of the origin, there is a positive number $a_{0}$ such that the $n$-simplex $\Delta_{0}$ generated by the origin and the points $a_{0} \mathbf{e}_{i}$ is contained in $V$. Thus, $\tilde{\Delta}_{0}=\varphi^{-1}\left(\Delta_{0}\right)$ is an admissible region induced by the singular simplex $s_{0}:=\varphi^{-1} \circ\left(a_{0} \mathrm{Id}\right): \Delta^{n} \rightarrow \mathscr{S}$, where Id $: \Delta^{n} \rightarrow \mathbb{R}^{n}$ is the identity mapping. Similarly, for $a_{m}=2^{-m} a_{0}$, let $\Delta_{m}$ be the $n$-simplex generated by the origin and the points $a_{m} \mathbf{e}_{i}$, and set $\tilde{\Delta}_{m}=\varphi^{-1}\left\{\Delta_{m}\right\} \subset \mathscr{S}$. Let $\Delta_{m p}$ be the oriented simplices that make up $\partial \Delta_{m}$ and $\tilde{\Delta}_{m p}=\varphi^{-1}\left\{\Delta_{m p}\right\}$. We observe that $\tilde{\Delta}_{m}$ is the image of the singular simplex $s_{m}=\varphi^{-1} \circ\left(a_{m} \mathrm{Id}\right): \Delta^{n} \rightarrow \mathscr{S}$ and the faces $\tilde{\Delta}_{m p}$ are the images of the singular simplices $s_{m p}=\varphi^{-1} \circ\left(a_{m} \mathrm{Id}\right) \circ \kappa_{p}: \Delta^{n-1} \rightarrow \mathscr{S}$, with $\partial s_{m}=\sum_{p} s_{m p}$.

7.4.3. Construction. Using the boundedness assumption for $\tilde{\Delta}_{m}$, for all $m=0,1,2, \ldots$, we have,

$$
\left|\sum_{p} \int_{\tilde{\Delta}_{m p}} \tau_{\tilde{\Delta}_{m p}}\right|=\left|\sum_{p} \int_{s_{m p}} \tau_{\tilde{\Delta}_{m p}}\right| \leqslant \int_{\tilde{\Delta}_{m}} \zeta,
$$

where $\tau_{\tilde{\Delta}_{m p}}$ is the restriction of $\tau_{\tilde{\Delta}_{m}}$ to the $p$-th face. Using integration on singular simplices

$$
\int_{\tilde{\Delta}_{m}} \varsigma=\int_{s_{m}} \varsigma=\int_{\Delta^{n}}\left(T s_{m}\right)^{*}(\varsigma)_{1 \ldots, n} \mathrm{~d} x^{1} \cdots \mathrm{d} x^{n} .
$$

By the mean value theorem for integration, there is a point $\mathbf{q}_{m} \in \Delta^{n}$ such that

$$
\int_{\Delta^{n}}\left(T s_{m}\right)^{*}(\varsigma)_{1 \ldots, n} \mathrm{~d} x^{1} \cdots \mathrm{d} x^{n}=\frac{1}{n !}\left(T s_{m}\right)^{*}(\varsigma)_{1 \ldots, n}\left(\mathbf{q}_{m}\right),
$$

where $1 / n$ ! is the measure of the $n$-simplex. Using $x_{m}=s_{m}\left(\mathbf{q}_{m}\right)=\varphi^{-1} \circ\left(a_{m} \operatorname{Id}\right)\left(\mathbf{q}_{m}\right)$, we have

$$
\begin{aligned}
\left(T s_{m}\right)^{*}(\varsigma)_{1 \ldots, n}\left(\mathbf{q}_{m}\right) \\
=\left(T s_{m}\right)^{*}(\varsigma)\left(\mathbf{q}_{m}\right)\left(\mathbf{e}_{1}, \ldots, \mathbf{e}_{n}\right), \\
=\varsigma\left(x_{m}\right)\left[T_{\mathbf{q}_{m}}\left(\varphi^{-1} \circ\left(a_{m} \mathrm{Id}\right)\right)\left(\mathbf{e}_{1}\right), \ldots, T_{\mathbf{q}_{m}}\left(\varphi^{-1} \circ\left(a_{m} \mathrm{Id}\right)\right)\left(\mathbf{e}_{n}\right)\right] \\
=\varsigma\left(x_{m}\right)\left[T_{a_{m} \mathbf{q}_{m}} \varphi^{-1}\left(a_{m} \mathbf{e}_{1}\right), \ldots, T_{a_{m} \mathbf{q}_{m}} \varphi^{-1}\left(a_{m} \mathbf{e}_{n}\right)\right] \\
=a_{m}^{n} \varsigma\left(x_{m}\right)\left[T_{a_{m} \mathbf{q}_{m}} \varphi^{-1}\left(\mathbf{e}_{1}\right), \ldots, T_{a_{m} \mathbf{q}_{m}} \varphi^{-1}\left(\mathbf{e}_{n}\right)\right] \\
=a_{m}^{n}\left(T \varphi^{-1}\right)^{*}(\varsigma)\left(a_{m} \mathbf{q}_{m}\right)\left(\mathbf{e}_{1}, \ldots, \mathbf{e}_{n}\right),
\end{aligned}
$$

where we applied

$$
T_{\mathbf{q}_{m}}\left(\varphi^{-1} \circ\left(a_{m} \mathrm{Id}\right)\right)=T_{a_{m} \operatorname{Id}\left(\mathbf{q}_{m}\right)} \varphi^{-1} \circ T_{\mathbf{q}_{m}}\left(a_{m} \mathrm{Id}\right)=T_{a_{m} \mathbf{q}_{m}} \varphi^{-1} \circ\left(a_{m} \mathrm{Id}\right)
$$

which follows from Equation (5.25) and the linearity of $a_{m}$ Id. Thus,

$$
\int_{\tilde{\Delta}_{m}} \varsigma=\frac{a_{m}^{n}}{n !}\left(T \varphi^{-1}\right)^{*}(\varsigma)\left(a_{m} \mathbf{q}_{m}\right)\left(\mathbf{e}_{1}, \ldots, \mathbf{e}_{n}\right) .
$$


Similarly, using the mean value theorem for the integrals over $\tilde{\Delta}_{m p}$,

$$
\begin{aligned}
\int_{\tilde{\Delta}_{m p}} \tau_{\tilde{\Delta}_{m p}} & =\int_{\Delta^{n-1}}\left(T s_{m p}\right)^{*}\left(\tau_{\tilde{\Delta}_{m p}}\right)_{1 \ldots, n-1} \mathrm{~d} x^{1} \cdots \mathrm{d} x^{n-1}, \\
& =\frac{1}{(n-1) !}\left(T s_{m p}\right)^{*}\left(\tau_{\tilde{\Delta}_{m p}}\right)_{1 \ldots, n-1}\left(\mathbf{q}_{m p}\right),
\end{aligned}
$$

for some point $\mathbf{q}_{m p} \in \Delta^{n-1}$. With $x_{m p}=s_{m p}\left(\mathbf{q}_{m p}\right)=\varphi^{-1} \circ\left(a_{m} \mathrm{Id}\right) \circ \kappa_{p}\left(\mathbf{q}_{m p}\right)$, one has

$$
\begin{aligned}
&\left(T s_{m p}\right)^{*}\left(\tau_{\tilde{\Delta}_{m p}}\right) 1 \ldots, n-1\left(\mathbf{q}_{m p}\right) \\
&=\left(T s_{m p}\right)^{*}\left(\tau_{\tilde{\Delta}_{m p}}\right)\left(\mathbf{q}_{m p}\right)\left(\mathbf{e}_{1}, \ldots, \mathbf{e}_{n-1}\right), \\
&= \tau_{\tilde{\Delta}_{m p}}\left(x_{m p}\right)\left[T_{\mathbf{q}_{m p}}\left(\varphi^{-1} \circ\left(a_{m} \mathrm{Id}\right) \circ \kappa_{p}\right)\left(\mathbf{e}_{1}\right), \ldots\right. \\
&\left.\ldots, T_{\mathbf{q}_{m p}}\left(\varphi^{-1} \circ\left(a_{m} \mathrm{Id}\right) \circ \kappa_{p}\right)\left(\mathbf{e}_{n-1}\right)\right], \\
&=a_{m}^{n-1} \tau_{\tilde{\Delta}_{m p}}\left(x_{m p}\right)\left[T_{a_{m} \kappa_{p}\left(\mathbf{q}_{m p}\right)} \varphi^{-1}\left(\mathfrak{e}_{p 1}\right), \ldots\right. \\
&\left.\ldots, T_{a_{m} \kappa_{p}\left(\mathbf{q}_{m p}\right)} \varphi^{-1}\left(\mathfrak{e}_{p n-1}\right)\right] .
\end{aligned}
$$

To arrive at the third equality above we used

$$
\begin{aligned}
T_{\mathbf{q}_{m p}}\left(\varphi^{-1} \circ\left(a_{m} \mathrm{Id}\right) \circ \kappa_{p}\right)\left(\mathbf{e}_{l}\right) & =T_{a_{m} \kappa_{p}\left(\mathbf{q}_{m p}\right)} \varphi^{-1} \circ T_{\kappa_{p}\left(\mathbf{q}_{m p}\right)}\left(a_{m} \mathrm{Id}\right) \circ T_{\mathbf{q}_{m p}} \kappa_{p}\left(\mathbf{e}_{l}\right), \\
& =a_{m} T_{a_{m} \kappa_{p}\left(\mathbf{q}_{m p}\right)} \varphi^{-1} \circ T \kappa_{p}\left(\mathbf{e}_{l}\right), \\
& =a_{m} T_{a_{m} \kappa_{p}\left(\mathbf{q}_{m p}\right)} \varphi^{-1}\left(\mathfrak{e}_{p l}\right),
\end{aligned}
$$

noting that $T_{\mathbf{q}_{m}} \kappa_{p}$ is independent of the point where it is evaluated and clearly, $T_{\kappa_{p}\left(\mathbf{q}_{m p}\right)}\left(a_{m} \mathrm{Id}\right)=a_{m} \mathrm{Id}$.

The balance equation (7.20) for the simplices $\tilde{\Delta}_{m}$ assumes the form

$$
\begin{array}{r}
\left|\sum_{p} \frac{a_{m}^{n-1}}{(n-1) !} \tau_{\tilde{\Delta}_{m p}}\left(x_{m p}\right)\left[T_{a_{m} \kappa_{p}\left(\mathbf{q}_{m p}\right)} \varphi^{-1}\left(\mathfrak{e}_{p 1}\right), \ldots, T_{a_{m} \kappa_{p}\left(\mathbf{q}_{m p}\right)} \varphi^{-1}\left(\mathfrak{e}_{p n-1}\right)\right]\right| \\
\leqslant a_{m}^{n}\left(T \varphi^{-1}\right)^{*}(\varsigma)\left(a_{m} \mathbf{q}_{m}\right)\left(\mathbf{e}_{1}, \ldots, \mathbf{e}_{n}\right) .
\end{array}
$$

Dividing both sides of the inequality above by $a_{m}^{n-1}$, we take the limit of the equation as $m \rightarrow \infty$. Noting that $\lim _{m \rightarrow \infty} a_{m}=0, \lim _{m \rightarrow \infty} x_{m p}=x, \lim _{m \rightarrow \infty} a_{m} k_{p}\left(\mathbf{q}_{m p}\right)=$ 0 , one obtains from $\tau_{\tilde{\Delta}_{m p}}\left(x_{m p}\right)=\mathfrak{t}_{x_{m p}}$ and the regularity assumption that

$$
\sum_{p} \mathfrak{t}_{x}\left[T_{0} \varphi^{-1}\left(\mathfrak{e}_{p 1}\right), \ldots, T_{0} \varphi^{-1}\left(\mathfrak{e}_{p n-1}\right)\right]=0 .
$$

As $S_{p l}=T_{0}\left(\varphi^{-1}\right)\left(\mathfrak{e}_{p l}\right)$, see (7.I9), we can finally write

$$
\sum_{p} \mathfrak{t}_{x}\left(S_{p}\right)=0
$$


We conclude that all the assumptions made in Section 3.I are satisfied. It follows that at each point $x \in \mathscr{S}$ there is an $(n-1)$-alternating tensor $J(x)$ such that $\mathfrak{t}_{x}\left(v_{1}, \ldots, v_{n-1}\right)=J(x)\left(v_{1}, \ldots, v_{n-1}\right)$. Equations (7.6) and (7.7) follow.

7.5. The Differential Balance Law. By the regularity assumption the Cauchy mapping $t$ is smooth. In addition, it follows from Cauchy's theorem that the flux $(n-1)$-form $J: \mathscr{S} \rightarrow \bigwedge^{n-1}\left(T^{*} \mathscr{S}\right)$ satisfies $J(x)=\mathfrak{t}_{x}$, and it follows that $J$ is a smooth form. For a given region $\mathscr{R}$, using (7.6) in the balance equation (7.3) one obtains

$$
\int_{\partial \mathscr{R}} T \iota_{\partial \mathscr{R}}^{*}(J)+\int_{\mathscr{R}} \beta=\int_{\mathscr{R}} \varsigma .
$$

We can apply Stokes's theorem to the boundary integral above so that

$$
\int_{\mathscr{R}} \mathrm{d} J+\int_{\mathscr{R}} \beta=\int_{\mathscr{R}} \varsigma
$$

Since the equation above holds for an arbitrary region, we conclude that the balance equation is equivalent to

$$
\mathrm{d} J+\beta=\varsigma
$$

- the differential balance law.

\section{Properties of Fluxes}

8.I. Notes on Orientation. We collect here some notes regarding the orientation aspects of the foregoing analysis.

(I) We interpret $\tau_{\mathscr{R}}(x)\left(v_{1}, \ldots, v_{n-1}\right)$ as the infinitesimal flux of the property out of the region $\mathscr{R}$ through the infinitesimal oriented simplex induced by the positively oriented collection $v_{1}, \ldots, v_{n-1}$. In case the collection of vectors is negatively oriented, $\tau_{\mathscr{R}}(x)\left(v_{1}, \ldots, v_{n-1}\right)$ is interpreted as the infinitesimal flux into the region.

(2) We defined

$$
\mathfrak{t}_{x}\left(v_{1}, \ldots, v_{n-1}\right)=\tau_{\mathscr{R}}(x)\left(v_{1}, \ldots, v_{n-1}\right)
$$

for any region $\mathscr{R}$ such that the collection $v_{1}, \ldots, v_{n-1} \in T_{x} \partial \mathscr{R}$ are positively oriented. Thus, the order in which the collection is written, specifies the orientation for which $\mathfrak{t}_{x}$ applies. At this point, for an odd permutation $\pi$, there is no relation between $\mathfrak{t}_{x}\left(v_{1}, \ldots, v_{n-1}\right)$ and $\mathfrak{t}_{x}\left(v_{\pi_{1}}, \ldots, v_{\pi_{n-1}}\right)$.

(3) Assume that $\mathscr{R}$ and $\mathscr{R}^{\prime}$ are two regions whose boundaries are tangent at $x \in$ $\mathscr{S}$,i.e., $H=T \iota_{\partial \mathscr{R}}\left\{T_{x} \partial \mathscr{R}\right\}=T \iota_{\partial \mathscr{R}^{\prime}}\left\{T_{x} \partial \mathscr{R}^{\prime}\right\}$. Assume also that the regions $\mathscr{R}$ and $\mathscr{R}^{\prime}$ induce opposite orientations on the hyperplane $H$. Consider the collection of tangent vectors $v_{1}, \ldots, v_{n-1} \in H$ which is positively oriented relative to $\partial \mathscr{R}$ and negatively oriented relative to $\partial \mathscr{R}^{\prime}$. It follows that for 
an odd permutation $\pi$

$$
\begin{aligned}
\tau_{\mathscr{R}}(x)\left(v_{1}, \ldots, v_{n-1}\right) & =\mathfrak{t}_{x}\left(v_{1}, \ldots, v_{n-1}\right), \\
& =J(x)\left(v_{1}, \ldots, v_{n-1}\right), \\
& =-J(x)\left(v_{\pi_{1}}, \ldots, v_{\pi_{n-1}}\right), \\
& =-\mathfrak{t}_{x}\left(v_{\pi_{1}}, \ldots, v_{\pi_{n-1}}\right), \\
& =-\tau_{\mathscr{R}^{\prime}}(x)\left(v_{\pi_{1}}, \ldots, v_{\pi_{n-1}}\right),
\end{aligned}
$$

where in the last line we used the assumption that $v_{\pi_{1}}, \ldots, v_{\pi_{n-1}}$ are positively oriented relative to $\partial \mathscr{R}^{\prime}$. This result exhibits the way that balance implies that the flux density out of $\mathscr{R}$ is the flux density entering $\mathscr{R}^{\prime}$. In the vector valued case this property implies Newton's law of action and reaction.

(4) The foregoing observations motivate the engineering practice, used mainly for internal forces) to associate with a hyperplane a pair of opposite fluxes $(\tau,-\tau)$, which apply to the two possible orientations of the hyperplane.

8.2. Kinetic Fluxes and Kinematic Fluxes. We describe here some of the properties of flux $(n-1)$-forms that generalize familiar properties of flux vector fields in the case where an inner product of vectors is available. First it is shown, how a volume element, a weaker geometric structure than a Riemannian metric, enables one to represent the flux form by a vector field.

Let $\theta$ be a volume element on $\mathscr{S}$. Locally, for a positively oriented chart in $\mathscr{S}, \theta$ is of the form

$$
\theta_{1 \ldots n}\left(x^{i}\right) \mathrm{d} x^{1} \wedge \cdots \wedge \mathrm{d} x^{n}
$$

where $\theta_{1 \ldots n}\left(x^{i}\right)>0$. Let $v: \mathscr{S} \rightarrow T \mathscr{S}$ be a vector field in $\mathscr{S}$, represented locally by $\sum_{i} v^{i}(x) \partial / \partial x^{i}$, then, using Example 4.6, the $(n-1)$-form $\left.\left.v\right\lrcorner \theta,(v\lrcorner \theta\right)(x)=$ $v(x)\lrcorner \theta(x)$ is represented locally by

$$
\sum_{k}(-1)^{k-1} \theta_{1 \ldots n} v^{k} \mathrm{~d} x^{1} \wedge \cdots \wedge \widehat{\mathrm{d} x^{k}} \wedge \ldots \mathrm{d} x^{n} .
$$

Let $J$ be an $(n-1)$-flux form. The condition that $J=v\lrcorner \theta$ may be written for the local representation $\sum_{k} J_{1 \ldots \widehat{k} \ldots n} \mathrm{~d} x^{1} \wedge \ldots \wedge \widehat{\mathrm{d} x^{k}} \wedge \ldots \mathrm{d} x^{n}$ as

$$
J_{1 \ldots \widehat{k} \ldots n}=(-1)^{k-1} \theta_{1 \ldots n} v^{k} .
$$

Since $\theta_{1 \ldots, n}>0$, given $J$, there is a unique vector field $v$ given locally by

$$
v^{k}=(-1)^{k-1} \frac{J_{1 \ldots \widehat{k} \ldots n}}{\theta_{1 \ldots n}} .
$$

In other words, the volume element $\theta$ induces a vector bundle isomorphism

$$
\iota_{\theta}: \bigwedge^{n-1}\left(T^{*} \mathscr{S}\right) \longrightarrow T \mathscr{S}
$$

whose inverse is given by $\left.\iota_{\theta}^{-1}(v)=v\right\lrcorner \theta$.

If a positive extensive property is under consideration, such as mass or entropy, the density $\rho$ of the property, a positive $n$-form, may be used as a volume element. It follows that in such a case the vector field $w=\iota_{\rho}(J)$ is physically meaningful. For 
example, for the case of the mass property, $J$ indicates the flux of mass and $w=$ $\iota_{\rho}(J)$, obtained by dividing the components of the mass flux (with the appropriate sign) by the mass density, models the velocity of the mass particles. Therefore, for a case where the volume element used is the positive density of the property under consideration, it is natural to refer to $w=\iota_{\rho}(J)$ as the kinematic flux, distinguishing it from the kinetic flux $J$.

For a given volume element $\theta$, let $w=\iota_{\theta}(J)$ so that $\left.J=w\right\lrcorner \theta$. Let $v_{1}, \ldots, v_{n-1}$ be linearly independent (or else the flux through the simplex $\left[v_{1}, \ldots, v_{n-1}\right]$ will vanish independently of $J$ ). Since $\theta\left(u_{1}, \ldots, u_{n}\right)$ does not vanish if and only if $u_{1}, \ldots, u_{n}$ are linearly independent,

$$
J\left(v_{1}, \ldots, v_{n-1}\right)=\theta\left(w, v_{1}, \ldots, v_{n-1}\right)=0
$$

if and only if $w$ may be represented as a linear combination of $v_{1}, \ldots, v_{n-1}$. We conclude that the flux through the simplex $\left[v_{1}, \ldots, v_{n-1}\right]$ vanishes if and only if the subspace that the vectors span contains the kinematic flux $w=\iota_{\theta}(J)$.

8.3. The Flux Bundle. We observe that the dependence of $v=\iota_{\theta}(J)$ on the choice of volume element $\theta$ as exhibited in Equation (8.6) is via multiplication by a positive constant. It follows that the flux form $J$ induces a I-dimensional subbundle of the tangent bundle $F_{J}$ such that

$$
\left(F_{J}\right)_{x}=\left\{\iota_{\theta(x)}(J(x)) \mid \theta(x) \in \bigwedge^{n}\left(T_{x} \mathscr{S}\right)\right\} .
$$

Using (8.6), we may write locally

$$
\left(F_{J}\right)_{x}=\left\{a \sum_{k}(-1)^{k-1} J_{1 \ldots \widehat{k} \ldots n} \frac{\partial}{\partial x^{k}} \mid a \in \mathbb{R}\right\} .
$$

The orientation of $\mathscr{S}$ as reflected in the sign of $\theta_{1 \ldots n}$ for a positively oriented chart, induces an orientation in $F_{J}$. We will refer to $F_{J}$ as the flux bundle associated with $J$. It is emphasized that the flux bundle is independent of the volume element.

Since for $a \in \mathbb{R}, a \neq 0$, the vector $a w$ belongs to $\operatorname{span}\left\{v_{1}, \ldots, v_{n-1}\right\}$ if and only if $w \in \operatorname{span}\left\{v_{1}, \ldots, v_{r}\right\}$, it follows that the flux through a simplex $\left[v_{1}, \ldots, v_{n-1}\right]$ vanishes if and only if $\left(F_{J}\right)_{x}$ is a subspace of $\operatorname{span}\left\{v_{1}, \ldots, v_{n-1}\right\}$. Furthermore, if $\mathscr{R}$ is a region, a collection of linearly independent vectors $v_{1}, \ldots, v_{n-1} \operatorname{span} T_{x} \partial \mathscr{R}$. It follows from the generalized Cauchy formula $\tau_{\mathscr{R}}=T \iota_{\partial \mathscr{R}}^{*}(J)$ that $\tau_{\mathscr{R}}(x)$ vanishes if and only if the flux space $\left(F_{J}\right)_{x}$ is a subspace of $T_{x} \partial \mathscr{R}$. This is analogous to the situation in the context of the classical formulation where the flux density though a surface element vanishes if and only if the flux vector field is tangent to the surface element.

8.4. Flow Potentials and Stream Functions. For the case where $\beta=\varsigma=0$, the differential balance law (7.34) assumes the form $\mathrm{d} J=0$. A differential form $J$ for which $\mathrm{d} J=0$ is called closed. It follows from $\mathrm{d}^{2} \alpha=0$ (5.54) that for any $(n-2)$ form $\alpha, J=\mathrm{d} \alpha$ satisfies the differential balance equation. In such a case, we will refer to $\alpha$ as the potential or stream function for the flux field $J$. This way, the Maxwell 2 -form of electrodynamics induces the flux of the electric charge in the spacetime 
formulation (see Section II). A form $J$ is exact if it is given as $J=\mathrm{d} \alpha$ for some form $\alpha$. Thus, every exact form is closed. Locally, the Poincarè Lemma (e.g., [AMR88, pp. 435-437]) states that in some neighborhood of each point, every closed form is exact. However, the problem of existence of a stream function for $J$ defined on all of $\mathscr{S}$ is related to the global topological structure of the manifold. If the manifold may be smoothly deformed into a point, every closed form is exact.

Clearly, the potential form $\alpha$ is not unique, using $\mathrm{d}^{2} \lambda=0$, if an $r$-form $\omega$ is exact, i.e., $\omega=\mathrm{d} \alpha$ for an $(r-1)$-potential form $\alpha$, then, for any $(r-2)$-form $\lambda, \alpha^{\prime}=\alpha+\mathrm{d} \lambda$ is also a potential.

\section{Frames, Body Points, and Spacetime Structure}

9.I. Frames, Balance and Fluxes in Spacetime. The discussion so far was restricted to the case where the $(n+1)$-dimensional spacetime manifold $\mathscr{E}$ was modeled as a Cartesian product of a time manifold $\mathscr{T}=\mathbb{R}$ and an $n$-dimensional space manifold $\mathscr{S}$, i.e., $\mathscr{E}=\mathscr{T} \times \mathscr{S}$. This is a very restrictive assumption that contradicts even the Galilean point of view of physics. In a more general situation, one assumes the existence of local frames. A local frame $\Phi$ is an embedding

$$
\Phi: U \subset \mathscr{E} \longrightarrow \mathscr{T} \times \mathscr{S}
$$

where $U$ is an open subset of $\mathscr{E}$. A frame $\Phi$ is global in case $U=\mathscr{E}$. In the sequel we will denote by $\Phi_{\mathscr{T}}$ and $\Phi_{\mathscr{S}}$ the two components of the frame and will write $(t, x)$ for $\Phi(e), e \in \mathscr{E}$. The domains of the local frames are assumed to cover $\mathscr{E}$. It follows that the foregoing analysis applies in the image of the a local frame.

In general, a local chart $(\varphi, U)$ on the spacetime manifold $\mathscr{E}$ provides local world coordinates $\left(z^{1}, \ldots, z^{n+1}\right)$. For the case where a local frame $\Phi$ is given, the local representative $\tilde{\Phi}$ of $\Phi$, is of the form $\left(z^{1}, \ldots, z^{n+1}\right) \mapsto\left(t, x^{1}, \ldots, x^{n}\right)$, where $\left(x^{1}, \ldots, x^{n}\right)$ are local coordinates in $\mathscr{S}$. In the sequel, given a frame $\Phi$, we will refer to $\left(z^{1}, \ldots, z^{n+1}\right)$ as the frame coordinates if $z^{1}=t$ is the time coordinate and for $i>1, z^{i}=x^{i-1}$ are local coordinates in some chart in $\mathscr{S}$.

The tangent mapping $T \Phi: T \mathscr{E} \rightarrow T \mathscr{T} \times T \mathscr{S}$ has two components $T \Phi_{\mathscr{T}}$ and $T \Phi_{\mathscr{S}}$. For $u \in T_{e} \mathscr{E}$, we will write $u_{\mathscr{T}}=T \Phi_{\mathscr{T}}(u), u_{\mathscr{S}}=T \Phi_{\mathscr{S}}(u)$. As $T_{t} \mathscr{T}=\mathbb{R}$, $T_{t}^{*} \mathscr{T} \cong \mathbb{R}$, the natural base vector of $T_{t}^{*} \mathscr{T}, t \in \mathscr{T}$, is the number I, and $T_{e}^{*} \Phi_{\mathscr{T}}(1)$ will be denoted by $\mathrm{d} t$. Writing $\partial / \partial t=\left(T_{e} \Phi\right)^{-1}(1,0)$ it is noted that $\mathrm{d} t(\partial / \partial t)=$ $1\left(T_{e} \Phi_{\mathscr{T}}(\partial / \partial t)\right)=1$ and $\mathrm{d} t\left(T_{e}^{-1} \Phi(0, v)\right)=1\left(T_{e} \Phi_{\mathscr{T}}\left(T_{e}^{-1} F(0, v)\right)\right)=0$, etc. For an alternating tensor $\varphi \in \wedge^{r} T_{x} \mathscr{S}$ we set $\varphi=T_{e}^{*} \Phi \mathscr{S}(\varphi)$. Note that

$$
\begin{aligned}
\left.\frac{\partial}{\partial t}\right\lrcorner \underset{\sim}{\varphi}\left(v_{1}, \ldots, v_{r-1}\right) & =\underset{\sim}{\varphi}\left(\frac{\partial}{\partial t}, v_{1}, \ldots, v_{r-1}\right), \\
& =\varphi\left(T_{e} \Phi \mathscr{S}\left(\left(T_{e} \Phi\right)^{-1}(1,0)\right), T_{e} \Phi_{\mathscr{S}}\left(v_{1}\right), \ldots, T_{e} \Phi_{\mathscr{S}}\left(v_{r-1}\right)\right), \\
& =0 .
\end{aligned}
$$


Thus, given a global frame $\Phi$, a time-dependent $r$-form $\varphi, \varphi(t) \in \Omega^{r}(\mathscr{S})$, induces a form $\mathfrak{f}=\mathrm{d} t \wedge \varphi \in \Omega^{r+1}(\mathscr{E})$. Using Equation (4.89) as well as the previous equation,

$$
\left.\left.\frac{\partial}{\partial t}\right\lrcorner \mathfrak{f}=\frac{\partial}{\partial t}\right\lrcorner(\mathrm{d} t \wedge \varphi)=\varphi .
$$

This procedure may now be used to define $\mathfrak{b}=\mathrm{d} t \wedge \underset{\sim}{\beta}, \mathfrak{s}=\mathrm{d} t \wedge \xi$. In addition, let the spacetime flux be defined by

$$
\mathfrak{J}=-\mathrm{d} t \wedge L+\rho,
$$

where it is recalled that $\rho$ is the density of the extensive property under consideration. Let $J$ and $\rho$ be represented locally in the forms

$$
\sum_{k} J_{1 \ldots . . . . n} \mathrm{~d} x^{1} \wedge \cdots \wedge \widehat{\mathrm{d} x^{k}} \wedge \cdots \wedge \mathrm{d} x^{n}
$$

and $\rho_{1 . . . n} \mathrm{~d} x^{1} \wedge \cdots \wedge \mathrm{d} x^{n}$, then, by Equation (4.II2) $\underset{\sim}{ }$ and $\underset{\sim}{\rho}$ are represented locally as

$$
\sum_{k} J_{1 \ldots \widehat{k} \ldots n} \mathrm{~d} x^{1} \wedge \cdots \wedge \widehat{\mathrm{d} x^{k}} \wedge \cdots \wedge \mathrm{d} x^{n}
$$

and $\rho_{1 \ldots n} \mathrm{~d} x^{1} \wedge \cdots \wedge \underline{\mathrm{d}}^{n}$, respectively. It follows that the local representation of $\mathfrak{J}$ is of the form

$$
-\sum_{k} J_{1 \ldots . . . . n} \mathrm{~d} t \wedge \mathrm{d}^{1} \wedge \cdots \wedge \widehat{\widehat{\mathrm{d} x^{k}}} \wedge \cdots \wedge \mathrm{d} x^{n}+\rho_{1 \ldots n} \mathrm{~d} x^{1} \wedge \cdots \wedge \mathrm{d} x^{n} .
$$

In terms of frame coordinates, the local expression (9.5) for $\mathfrak{J}$ is

$$
-\sum_{k=2}^{n+1} J_{1 \ldots \widehat{k-1} \ldots n} \mathrm{~d} z^{1} \wedge \mathrm{d} z^{2} \wedge \cdots \wedge \widehat{\mathrm{d} z^{k}} \wedge \cdots \wedge \mathrm{d} z^{n+1}+\rho_{1 \ldots n} \mathrm{~d} z^{2} \wedge \cdots \wedge \mathrm{d} z^{n+1} .
$$

so that

$$
\mathfrak{J}_{2 \ldots n+1}=\rho_{1 \ldots n}, \quad \text { and } \quad \mathfrak{J}_{1 \ldots \widehat{k} \ldots n+1}=-J_{1 \ldots \widehat{k-1} \ldots n}, \text { for } k>1 .
$$

To compare the role played by $\mathfrak{J}$ in comparison with that of $J$, consider a region $\mathfrak{R} \subset \mathscr{E}$ with boundary $\partial \mathfrak{R}$. Consider $e \in \partial \mathfrak{R}$ and assume that $T \Phi\left\{T_{e} \partial \mathfrak{R}\right\}=\{t\} \times$ $T_{x} \mathscr{S}$, i.e., the tangent space to the boundary is "spacelike". Then, for $v_{1}, \ldots, v_{n} \in$ $T_{e} \partial \Re$,

$$
\begin{aligned}
\mathfrak{J}\left(v_{1}, \ldots, v_{n}\right) & =\underset{\sim}{\rho}\left(v_{1}, \ldots, v_{n}\right)-(\mathrm{d} t \wedge \underset{\sim}{J})\left(v_{1}, \ldots, v_{n}\right), \\
& =\underset{\sim}{\rho}\left(v_{1}, \ldots, v_{n}\right), \\
& =\rho\left(T \Phi_{\mathscr{S}}\left(v_{1}\right), \ldots, T \Phi_{\mathscr{S}}\left(v_{n}\right)\right),
\end{aligned}
$$

where the second term in the first line vanishes because $\mathrm{d} t\left(v_{i}\right)=0$, for all $i=$ $1, \ldots, n$. On the other hand, if $T_{e} \partial \Re$ contains $\partial / \partial t,\left\{T \Phi_{\mathscr{S}}\left(v_{1}\right), \ldots, T \Phi_{\mathscr{S}}\left(v_{n}\right)\right\}$ cannot contain $n$-linearly independent vectors so that $\underset{\sim}{\rho}\left(v_{1}, \ldots, v_{n}\right)=0$ and

$$
\mathfrak{J}\left(v_{1}, \ldots, v_{n}\right)=-(\mathrm{d} t \wedge \underset{\sim}{J})\left(v_{1}, \ldots, v_{n}\right) .
$$


In case $v_{i}=\partial / \partial t$,

$$
\begin{aligned}
\mathfrak{J}\left(v_{1}, \ldots, v_{n}\right) & =-(-1)^{i-1}(\mathrm{~d} t \wedge \underset{\sim}{J})\left(v_{i}, v_{1}, \ldots, \widehat{v}_{i}, \ldots, v_{n}\right), \\
& \left.=(-1)^{i} \frac{\partial}{\partial t}\right\lrcorner(\mathrm{d} t \wedge \underset{\sim}{J})\left(v_{1}, \ldots, \widehat{v}_{i}, \ldots, v_{n}\right), \\
& =(-1)^{i} \underset{\sim}{J}\left(v_{1}, \ldots, \widehat{v}_{i}, \ldots, v_{n}\right), \\
& \left.=(-1)^{i} J\left(T \Phi_{\mathscr{S}}\left(v_{1}\right), \ldots, T \widehat{\Phi_{\mathscr{S}}\left(v_{i}\right.}\right), \ldots, T \Phi_{\mathscr{S}}\left(v_{n}\right)\right) .
\end{aligned}
$$

Thus, the spacetime flux $\mathfrak{J}$ provides the flux density in accordance with the flux field $J$.

Next, we present the way the balance equations are written in term of the spacetime forms. First, it is noted that using Equations (5.62) and (5.54)

$$
\begin{aligned}
\mathrm{d} \mathfrak{J} & =\mathrm{d}(-\mathrm{d} t \wedge \underset{\sim}{J})+\mathrm{d} \rho, \\
& =\mathrm{d} t \wedge \mathrm{d} J+{ }_{\sim}+{ }_{\sim} \rho
\end{aligned}
$$

Since $\rho$ is represented locally in the form $\rho_{1 \ldots n} \mathrm{~d} x^{1} \wedge \cdots \wedge \mathrm{d} x^{n}$, the exterior derivative $\mathrm{d} \rho$ is represented by

$$
\frac{\partial \rho_{1} \ldots n}{\partial t} \mathrm{~d} t \wedge \mathrm{d} x^{1} \wedge \cdots \wedge \mathrm{d} x^{n},
$$

and by the definition of $\beta$ as the time derivative of $\rho$ we obtain

$$
\mathrm{d} \rho=\mathrm{d} t \wedge \underline{\beta}=\mathfrak{b} .
$$

Finally, the differential balance equation, (7.34) yields the spacetime version

$$
\mathrm{d} \mathfrak{J}=\mathfrak{s} .
$$

Integrating the balance equation in spacetime over a region $\Re \subset \mathscr{E}$ and using Stokes's theorem, the integral version of the balance in spacetime is

$$
\int_{\partial \mathfrak{R}} i_{\partial \mathfrak{R}}^{*}(\mathfrak{J})=\int_{\mathfrak{R}} \mathfrak{s}
$$

It is natural therefore to set

$$
\mathfrak{t}_{\mathfrak{K}}=\iota_{\partial \mathfrak{R}}^{*}(\mathfrak{J})
$$

and rewrite the integral balance equation in spacetime in the form

$$
\int_{\partial \mathfrak{R}} \mathfrak{t}_{\mathfrak{R}}=\int_{\mathfrak{R}} \mathfrak{s} .
$$

We conclude that for any given frame the equations of balance in spacetime have the same form as the equations of balance in space, and in fact, they are simpler in the sense that there is no counterpart to the term containing $\beta$. The relations between the various spacetime forms on $\mathscr{E}$ and the corresponding forms on $\mathscr{S}$ make no sense if we do not have a specific frame. However, if one postulates the balance equation (9.17) in spacetime, the procedure of Section 7.4 may be repeated yielding the existence of the $n$-form $\mathfrak{J}$ satisfying Equations (9.I6) and (9.I4) independently of any frame. It is also noted that flux fields on $\mathscr{E}$ are naturally time-dependent. 
9.2. Worldlines. Let $\theta$ be a volume element in spacetime and let $\mathfrak{J}$ be a flux field on $\mathscr{E}$. Then, the isomorphism $\iota_{\theta}$ of Equation (8.7) induces a vector field $w$ on $\mathscr{E}$ such that $w\lrcorner \theta=\mathfrak{J}$. We will refer to an integral line of $w$ as a worldline corresponding to the property $p$ for which $\mathfrak{J}$ is the flux. Clearly, each worldline is a one-dimensional submanifold of $\mathscr{E}$. We want to show that as a submanifold, a worldline is independent of the choice of volume element. Let $\theta^{\prime}$ be another volume element. Then, if the local representation of $\theta$ is of the form $\theta_{1 \ldots n+1} \mathrm{~d} z^{1} \wedge \cdots \wedge \mathrm{d} z^{n+1}$, the local representative of $\theta^{\prime}$ is of the form $a\left(z^{i}\right) \theta_{1 \ldots n+1} \mathrm{~d} z^{1} \wedge \cdots \wedge \mathrm{d} z^{n+1}$, for a unique positive function $a: \mathscr{E} \rightarrow \mathbb{R}^{+}$. It follows from Equation (8.6) that the vector field $w^{\prime}=\iota_{\theta^{\prime}}(\mathfrak{J})$ is related to $w=\iota_{\theta}(\mathfrak{J})$ by $a w^{\prime}=w$. The local systems of differential equations for the integral lines corresponding to the two vector fields are

$$
\dot{c}^{i}(p)=w^{i}\left(c^{j}(p)\right) \quad \text { and } \quad \dot{c}^{\prime i}(p)=w^{\prime i}\left(c^{\prime j}(p)\right)=\frac{1}{a\left(c^{\prime j}(p)\right)} w^{i}\left(c^{\prime j}(p)\right)
$$

where a superimposed dot indicates derivatives relative to the parameter $p$. Thus, the equations for the worldlines corresponding to $\theta^{\prime}$ are

$$
a\left(c^{\prime j}(p)\right) \dot{c}^{\prime i}(p)=w^{i}\left(c^{\prime j}(p)\right) .
$$

For a solution $c^{i}(p)$ of the equation on the left of (9.I8), consider the reparametrization $p^{\prime}=p^{\prime}(p)$, satisfying the condition

$$
\frac{\mathrm{d} p^{\prime}}{\mathrm{d} p}=\frac{1}{a\left(c^{i}(p)\right)}
$$

so that the reparametrization is invertible and $\mathrm{d} p / \mathrm{d} p^{\prime}\left(p^{\prime}(p)\right)=a\left(c^{i}(p)\right)$. Let $\widehat{c}$ be the reparametrized curve $\hat{c}^{i}\left(p^{\prime}(p)\right)=c^{i}(p)$ so that

$$
\frac{\mathrm{d} \hat{c}^{i}}{\mathrm{~d} p^{\prime}}=a \frac{\mathrm{d} \hat{c}^{i}}{\mathrm{~d} p} \text {. }
$$

In view of the differential equations this may be written as

$$
\frac{\mathrm{d} \hat{c}^{i}}{\mathrm{~d} p^{\prime}}\left(p^{\prime}\right)=a\left(\hat{c}^{j}\left(p^{\prime}\right)\right) w\left(\hat{c}^{j}\left(p^{\prime}\right)\right) .
$$

Thus, the reparametrized solution $\hat{c}^{j}$ is the worldline of (9.19).

We conclude that a different choice of a volume element simply induces a reparametrization of the integral line. The tangent bundle of the worldline submanifold is clearly the flux bundle. In terms of the theory of distributions-sub-bundles of the tangent bundle - the one dimensional flux bundle is integrable, i.e., it is the tangent bundle of a one dimensional manifold.

Example 9.1. Consider the case where a frame is given on $\mathscr{E}$ and assume that $J=0$. It follows from (9.5) that $\mathfrak{J}$ is represented locally by

$$
\sum_{k=1}^{n+1} \mathfrak{J}_{1 \ldots \widehat{k} \ldots n+1}\left(z^{i}\right) \mathrm{d} z^{1} \wedge \cdots \wedge \widehat{\mathrm{d} z^{k}} \wedge \cdots \wedge \mathrm{d} z^{n+1}=\rho_{1 \ldots n}\left(z^{i}\right) \mathrm{d} z^{2} \wedge \cdots \wedge \mathrm{d} z^{n+1} .
$$


Thus,

$$
\mathfrak{J}_{1 \ldots \widehat{k} \ldots n+1}= \begin{cases}\rho_{1 \ldots n}, & k=1, \\ 0, & k>1 .\end{cases}
$$

Let $\theta$ be a volume element on $\mathscr{E}$. Using Equation (8.6), it follows that, independently of the choice of volume element, the last $n$ local differential equations for the integral lines are

$$
\frac{\mathrm{d} c^{k}}{\mathrm{~d} p}=0, \quad k>1,
$$

which imply that $c^{k}(p)=z_{0}^{k}, k>1$, where $z_{0}$ is the initial condition or the event where the integral line originates.

9.3. Material Points, the Material Universe and Material Frames. The notion of a material point is one of the basic elements of continuum mechanics. The existence of material points has been motivated by the conservation of mass and their basic properties are postulated in the principle of material impenetrability which in its elementary version implies that two distinct material points do not occupy the same event in spacetime. (See for example [Tru77].)

The foregoing discussion enables one to generalize the notion of a material point. For a given extensive property whose balance in spacetime is reflected by the flux $n$-form $\mathfrak{J}$ in $\mathscr{E}$, we will identify a material point with an integral line of the flux bundle induced by $\mathfrak{J}$. It is implied that material points may be defined for extensive properties other than mass and that they may be considered even in cases where the source term in the balance equation does not vanish. Thus, one could define material points for the electric charge, for a growing biological body and for the heat flux field, where the term "material point" is used only figuratively.

The collection of material points is traditionally referred to as the material universe. It would be desirable if the material universe had a structure of a differentiable manifold. Let $\theta$ be a volume element in $\mathscr{E}$. We first note that it follows from the result quoted in the last paragraph of Section 5.8 that for any point $e_{0} \in \mathscr{E}$ such that $\mathfrak{J}\left(e_{0}\right) \neq 0$ there is a chart $\left(\varphi, U_{0}\right)$ in a neighborhood of $e_{0}$ such that the vector field $\iota_{\theta}(\mathfrak{J})$ is represented locally by $\partial / \partial x^{1}$. Thus, the worldlines in $U_{0}$ may be represented in the form $z^{1}(p)=z_{0}^{1}+p, z^{i}(p)=z_{0}^{i}$, for $i>1$, where $z_{0}^{i}$ are the coordinates of $e_{0}$. We can assume without loss of generality that $\varphi\left\{U_{0}\right\}$ is convex. Consider the collection $F_{0}$ of of points in $U_{0}$ such each $e \in F_{0}$ is of the form $\left(z_{0}^{1}, z^{2}, \ldots, z^{n+1}\right)$. It follows that two distinct points on a single worldline cannot belong to $F_{0}$ and that $z^{2}, \ldots, z^{n+1}$ parametrize the various worldlines. Thus, regarding worldlines as material points, $F_{0}$ is a local $n$-dimensional submanifold of $\mathscr{E}$ which contains the material points in the neighborhood. Each event in $U_{0}$ may be represented by $(p, X), p \in(a, b), X \in F_{0}$. We may refer to the mapping $\Phi: U_{0} \subset \mathscr{E} \rightarrow(a, b) \times F_{0}$ as a local material frame. Clearly, the choice of volume element does not change the material frame in any significant way. It may only change the values determining the interval $(a, b)$.

Considering the material universe from the global point of view, we define the equivalence relation $e_{1} \sim e_{2}$ if $e_{1}$ and $e_{2}$ are on the same worldline so that an equivalence class is a material point. Thus, the material universe may be defined as the 
quotient set $\mathscr{E} / \sim$. The situation discussed above need not be extendable to a product of the material universe and the real axis. In other words, global material frames do not exist necessarily. For example, consider a vector field on the two-dimensional torus that winds around the torus an irrational number of times. In such a case, the flow "mixes" the material points in such a way that a manifold structure cannot be assigned to the collection of integral lines.

\section{IO. STREsses}

IO.I. Force Fields on ManifLolds. In the traditional formulation of continuum mechanics, set in a Euclidean space, one integrates the body force field and the surface force field to obtain their resultants. This is possible because in a Euclidean space one can transport vectors from one point to another and then add them up in a natural way. As mentioned in Section 5.3, for the general geometry of differential manifolds there is no natural way to compare tangent vectors belonging to two distinct tangent spaces. It follows that the basic definitions of force fields should be revised. The point of view adopted here is that the value of a force field at a point in space acts on the value of a generalized velocity field at that point to produce the power density. In accordance with classical mechanics of mass particles, the power depends linearly on the velocity. We will show how these general ideas lead to natural definitions of force fields for general manifolds.

The power density should be integrated over the appropriate manifold in order to yield the total power, a real number. Thus, the theory of integration of forms over manifolds implies that the power density over a manifold of dimension $d$ should be represented by a $d$-form over that manifold. Thus, for a region $\mathscr{R}$ in $\mathscr{S}$, a power density on $\mathscr{R}$ should be an $n$-form defined on $\mathscr{R}$ and a power density on $\partial \mathscr{R}$ should be modeled by an $(n-1)$-form on $\partial \mathscr{R}$.

Let $\mathscr{M}$ be a $d$-dimensional orientable manifold representing either the physical space $\mathscr{S}$ (assuming a particular frame is given) or the event world (spacetime) $\mathscr{E}$. As discussed in Example 5.7, we regard generalized velocity fields over $\mathscr{M}$ as sections of some given vector bundle $\pi: W \rightarrow \mathscr{M}$. In particular, for the case where $\mathscr{M}$ is either the physical space or spacetime, consider an extensive property for which the generalized Cauchy's postulates (Section 7.4) hold. Then, given a volume element, the kinematic flux, a vector field over the corresponding manifold, may serve as an example of a generalized velocity - the generalized velocity of the material points induced by the flux form as in Section 9.3. Furthermore, in order to abandon the dependence on the choice of volume element, the kinetic flux form itself may be taken as an example of a section representing a generalized velocity. In this case, a generalized velocity $w$ is a section of $W=\bigwedge^{n}\left(T^{*} \mathscr{E}\right)$, where $n+1$ is the dimension of $\mathscr{E}$.

Let $\mathscr{R}$ be a compact $d$-dimensional admissible region (a smooth orientable submanifold or a chain) in $\mathscr{M}$ with boundary $\partial \mathscr{R}$. As in Section 5.6, $L\left(W, \bigwedge^{d} T^{*} \mathscr{R}\right)$ denotes the vector bundle whose fiber at $x$ is the space of linear mappings $W_{x} \rightarrow$ $\bigwedge^{d} T_{x}^{*} \mathscr{R}$. In analogy with the scalar valued fluxes discussed in Section 7.I a body force field on $\mathscr{R}$ is a section $\mathbf{b}_{\mathscr{R}}: \mathscr{R} \rightarrow L\left(W, \bigwedge^{d}\left(T^{*} \mathscr{R}\right)\right)$ so that the total power expended 
by the body force for a velocity field $w: \mathscr{R} \rightarrow W$ is

$$
P=\int_{\mathscr{R}} \mathbf{b}_{\mathscr{R}}(w)
$$

A boundary force field, or a surface force field, on $\partial \mathscr{R}$ is a section

$$
\mathbf{t}_{\mathscr{R}}: \partial \mathscr{R} \rightarrow L\left(W, \bigwedge^{d-1}\left(T^{*} \partial \mathscr{R}\right)\right),
$$

where we wrote $W$ instead of $\left.W\right|_{\partial \mathscr{R}}$ for the sake of simplicity. Thus, for any $(d-1)$ dimensional submanifold $D$ of $\partial \mathscr{R}$, the power expended by the surface force for a velocity field $u$ defined on $\partial \mathscr{R}$ is given by

$$
P=\int_{D} \mathbf{t}_{\mathscr{R}}(u) .
$$

The total power expended by both the body force and surface force over the region $\mathscr{R}$ and its boundary for the virtual velocity field $w$ is therefore

$$
P=F_{\mathscr{R}}(w)=\int_{\mathscr{R}} \mathbf{b}_{\mathscr{R}}(w)+\int_{\partial \mathscr{R}} \mathbf{t}_{\mathscr{R}}(w) .
$$

Using the scheme of notation of Section 5.4.3, an element $w$ of $W$ is represented under a vector bundle chart in the forms $\left(x^{1}, \ldots, x^{d}, w^{1}, \ldots, w^{m}\right)$ and $\sum_{r} w^{r} g_{r}$, where $m$ is the dimension of the typical fiber of the vector bundle and $g_{r}$ are the local base vectors induced by the vector bundle chart. A section of $W$ is represented therefore in the forms

$$
\left(x^{1}, \ldots, x^{d}, w^{1}\left(x^{i}\right), \ldots, w^{m}\left(x^{i}\right)\right)
$$

and $\sum_{r} w^{r}\left(x^{i}\right) g_{r}$. An element $\mathbf{b}$ of $L\left(W, \bigwedge^{d}\left(T^{*} \mathscr{R}\right)\right)$, is represented by $\left(x^{i}, \mathbf{b}_{1 \ldots d r}\right)$ or

$$
\sum_{r} \mathbf{b}_{1 \ldots d r} \mathrm{~d} x^{1} \wedge \cdots \wedge \mathrm{d} x^{d} \otimes g^{r}
$$

so that $\mathbf{b}(w)$ is represented by $\left(x^{i}, \sum_{r} \mathbf{b}_{1 \ldots d r} w^{r}\right)$ or

$$
\sum_{r} \mathbf{b}_{1 \ldots d r} w^{r} \mathrm{~d} x^{1} \wedge \cdots \wedge \mathrm{d} x^{d}
$$

which gives the representation of body forces and their action. Similarly, for local coordinates $y^{l}$ on $\partial \mathscr{R}$, a surface force $\mathbf{t}$ is represented in the form $\left(y^{l}, \mathbf{t}_{1 \ldots(d-1) r}\left(y^{q}\right)\right.$ or

$$
\sum_{r} \mathbf{t}_{1 \ldots(d-1) r} \mathrm{~d} y^{1} \wedge \cdots \wedge \mathrm{d} y^{d-1} \otimes g^{r}
$$

so that $\mathbf{t}(u)$ is represented by $\left(y^{l}, \sum_{r} t_{1 \ldots(d-1) r} u^{r}\right)$ or

$$
\sum_{r} \mathbf{t}_{1 \ldots(d-1) r} u^{r} \mathrm{~d} y^{1} \wedge \cdots \wedge \mathrm{d} y^{d-1}
$$


IO.2. Vector-Valued Alternating Tensors and Forms. We now extend the discussion in Section 5.6 concerning vector valued forms. We will denote by $\wedge^{r}\left(\mathbf{V}^{*}, \mathbf{W}\right)$ the vector space of alternating $r$-multilinear mappings from the vector space $\mathbf{V}$ to the vector space $\mathbf{W}$. In particular, $\bigwedge^{r} \mathbf{V}^{*}=\bigwedge^{r}\left(\mathbf{V}^{*}, \mathbb{R}\right)$. Thus, if $\left\{g_{p}\right\}, p=1, \ldots, m$ is a basis of $\mathbf{W}$, so that each element of $\mathbf{W}$ is of the form $w=\sum_{p} w^{p} g_{p}$, then, $T \in \bigwedge^{r}\left(\mathbf{V}^{*}, \mathbf{W}\right)$, is represented by the collection of alternating real valued tensors $\left\{T^{p} \in \bigwedge^{r} \mathbf{V}^{*}\right\}$ as

$$
T\left(v_{1}, \ldots, v_{r}\right)=\sum_{p=1}^{m} T^{p}\left(v_{1}, \ldots, v_{r}\right) g_{p} .
$$

If $\left\{e_{i}\right\}_{i=1}^{n}$ is a basis of $\mathbf{V}$, the real valued tensor $T^{p}$ is represented as

$$
T^{p}=\sum_{(\alpha)} T_{\alpha_{1} \ldots \alpha_{r}}^{p} e^{\alpha_{1}} \wedge \cdots \wedge e^{\alpha_{r}}
$$

so that using Equation (4.27)

$$
T^{p}\left(v_{1}, \ldots, v_{r}\right)=\sum_{(\alpha), I} T_{\alpha_{1} \ldots \alpha_{r}}^{p} v_{1}^{i_{1}} \cdots v_{r}^{i_{r}} \varepsilon_{I}^{\alpha}
$$

and

$$
T\left(v_{1}, \ldots, v_{r}\right)=\sum_{p,(\alpha), I} T_{\alpha_{1} \ldots \alpha_{r}}^{p} v_{1}^{i_{1}} \cdots v_{r}^{i_{r}} \varepsilon_{I}^{\alpha} g_{p}
$$

If one considers $T \in \bigwedge^{r}\left(\mathbf{V}^{*}, \mathbf{W}^{*}\right)$ instead of $\bigwedge^{r}\left(\mathbf{V}^{*}, \mathbf{W}\right)$ so the dual basis $\left\{g^{p}\right\}$ is used, Equation (IO.8) is replaced by

$$
T\left(v_{1}, \ldots, v_{r}\right)=\sum_{p,(\alpha), I} T_{\alpha_{1} \ldots \alpha_{r} p} v_{1}^{i_{1}} \cdots v_{r}^{i_{r}} \varepsilon_{I}^{\alpha} g^{p} .
$$

Let $T \in \bigwedge^{r}\left(\mathbf{V}^{*}, \mathbf{W}^{*}\right)$, and $w \in \mathbf{W}$ so that for $v_{1}, \ldots, v_{r} \in \mathbf{V}, T\left(v_{1}, \ldots, v_{r}\right)(w) \in \mathbb{R}$ is given by

$$
T\left(v_{1}, \ldots, v_{r}\right)(w)=\sum_{p,(\alpha), I} T_{\alpha_{1} \ldots \alpha_{r} p} v_{1}^{i_{1}} \cdots v_{r}^{i_{r}} \varepsilon_{I}^{\alpha} w^{p} .
$$

One may consider the mapping

$$
T^{\operatorname{tr}}: \mathbf{W} \longrightarrow \bigwedge^{r} \mathbf{V}^{*}
$$

satisfying

$$
T^{\operatorname{tr}}(w)\left(v_{1}, \ldots, v_{r}\right)=T\left(v_{1}, \ldots, v_{r}\right)(w)=\sum_{p,(\alpha), I} T_{\alpha_{1} \ldots \alpha_{r} p} v_{1}^{i_{1}} \cdots v_{r}^{i_{r}} \varepsilon_{I}^{\alpha} w^{p} .
$$

From the component representation above, it is clear that $T^{\mathrm{tr}}$ is linear and that

$$
T^{\operatorname{tr}}(w)=\sum_{p,(\alpha)} T_{\alpha_{1} \ldots \alpha_{r} p} w^{p} e^{\alpha_{1}} \wedge \cdots \wedge e^{\alpha_{r}} .
$$

For any two vector spaces $\mathbf{V}_{0}$, and $\mathbf{W}_{0}$, the vector space of linear mappings $\mathbf{V}_{0}$ to $\mathbf{W}_{0}$ is

$$
L\left(\mathbf{V}_{0}, \mathbf{W}_{0}\right)=\mathbf{W}_{0} \otimes \mathbf{V}_{0}^{*}
$$


and so

$$
T^{\operatorname{tr}} \in L\left(\mathbf{W}, \bigwedge^{r} \mathbf{V}^{*}\right)=\bigwedge^{r} \mathbf{V}^{*} \otimes \mathbf{W}^{*}
$$

The transposition operator, $T \mapsto T^{\mathrm{tr}}$,

$$
\operatorname{tr}: \bigwedge^{r}\left(\mathbf{V}^{*}, \mathbf{W}^{*}\right) \longrightarrow L\left(\mathbf{W}, \bigwedge^{r} \mathbf{V}^{*}\right)=\bigwedge^{r} \mathbf{V}^{*} \otimes \mathbf{W}^{*}
$$

is clearly an isomorphism. For an element $S \in L\left(\mathbf{W}, \wedge^{r} \mathbf{V}^{*}\right)$, we will use the notation $S^{\mathrm{T}}=\operatorname{tr}^{-1}(S)$, so that

$$
S^{\top}\left(v_{1}, \ldots, v_{r}\right)(w)=S(w)\left(v_{1}, \ldots, v_{r}\right) .
$$

The foregoing discussion may be applied to the fibers of two vector bundles $V$ and $W$ over a manifold $\mathscr{M}$. Thus, a vector bundle valued differential form $T$ will be a section of $\bigwedge^{r}\left(V^{*}, W\right)$ whose fiber at $x \in \mathscr{M}$ is $\bigwedge^{r}\left(V_{x}^{*}, W_{x}\right)$. Extending Equation (IO.I6) to vector bundles we have the analog isomorphism

$$
\operatorname{tr}: \bigwedge^{r}\left(V^{*}, W^{*}\right) \longrightarrow L\left(W, \bigwedge^{r} V^{*}\right)=\bigwedge^{r} V^{*} \otimes W^{*}
$$

The definitions above may be applied to the body force field $\mathbf{b}_{\mathscr{R}}$, a section of $L\left(W, \bigwedge^{d}\left(T^{*} \mathscr{R}\right)\right)$, so that $\mathbf{b}_{\mathscr{R}}^{\top}$ is a section of $\bigwedge^{d}\left(T^{*} \mathscr{R}, W^{*}\right)$. While, $\mathbf{b}_{\mathscr{R}}(x)(w(x))$ is interpreted as the power density at $x, \mathbf{b}_{\mathscr{R}}^{\top}(x)\left(v_{1}, \ldots, v_{d}\right)$ may be interpreted as the resultant of the body force acting on the simplex defined by the vectors $v_{1}, \ldots, v_{d}$. The analogous observations hold for the surface force $\mathbf{t}_{\mathscr{R}} ; \mathbf{t}_{\mathscr{R}}^{\top}$ is a section of $\bigwedge^{d-1}\left(T^{*} \partial \mathscr{R}, W^{*}\right)$ and $\mathbf{t}_{\mathscr{R}}^{\top}(y)\left(v_{1}, \ldots, v_{d-1}\right)$ is interpreted as the resultant of the surface force acting on the simplex on the boundary at $y$ defined by the vectors $v_{1}, \ldots, v_{d-1}$.

It is noted that in general, there is no definition for the exterior derivative of differential forms valued in a vector bundle.

IO.3. Traction Stresses and Cauchy's Formula on Manifolds. This section introduces the analog of the Cauchy fluxes of Section 7.2 to the case of force fields. The analog of a flux form associated with a given extensive property is a traction stress field. A traction stress is a section $\sigma$ of $L\left(W, \bigwedge^{d-1} T^{*} \mathscr{M}\right)$. Thus, for a section $w$ of $W$, the $(d-1)$-form $\sigma(w), \sigma(w)(x)=\sigma(x)(w(x))$, is a flux field representing a flux of power. Given an oriented $(d-1)$-submanifold $D \subset \mathscr{M}$, the $(d-1)$-form $\sigma(w)$ may be restricted to vectors tangent to $D$ using the inclusion $\iota_{D}: D \rightarrow \mathscr{M}$ so that

$$
\rho_{D}=T \iota_{D}^{*}: \Omega^{d-1}(\mathscr{M}) \longrightarrow \Omega^{d-1}(D),
$$

with

$$
\rho_{D}(\sigma(u))\left(v_{1}, \ldots, v_{d-1}\right)=\sigma(u)\left(T \iota_{D}\left(v_{1}\right), \ldots, T \iota_{D}\left(v_{d-1}\right)\right) .
$$

The total power expended over $D$ for the vector field $u:\left.D \rightarrow W\right|_{D}$ is given by

$$
P=\int_{D} \rho_{D}(\sigma(u))
$$

Since the transposed $\sigma^{\top}$, as in (IO.I7), is a $W^{*}$-valued $(d-1)$-form satisfying

$$
\sigma^{\top}\left(v_{1}, \ldots, v_{d-1}\right)(w)=\sigma(w)\left(v_{1}, \ldots, v_{d-1}\right),
$$


one has

$$
\rho_{D}\left(\sigma^{\top}\right)\left(v_{1}, \ldots, v_{d-1}\right)=\sigma^{\top}\left(T \iota_{D}\left(v_{1}\right), \ldots, T \iota_{D}\left(v_{d-1}\right)\right) \in W^{*} .
$$

It follows that for a region $\mathscr{R}$ in $\mathscr{M}, \rho_{\partial \mathscr{R}}\left(\sigma^{\top}\right)$ is a $W^{*}$-valued $(d-1)$-form on $\partial \mathscr{R}$.

We conclude that a traction stress $\sigma$ induces on $\partial \mathscr{R}$ a surface force field $\mathbf{t}_{\mathscr{R}}$ by

$$
\mathbf{t}_{\mathscr{R}}^{\mathrm{T}}=\rho_{\partial \mathscr{R}}\left(\sigma^{\mathrm{\top}}\right)
$$

- the Cauchy formula for traction stresses. We note that Cauchy's formula (I0.24) is equivalent to the statement that for each vector field $u$,

$$
\mathbf{t}_{\mathscr{R}}(u)=\rho_{\partial \mathscr{R}}(\sigma(u)) .
$$

In analogy with the situation for scalar valued fluxes in Section 8.I, we observe that the section $\mathbf{t}: D \rightarrow L\left(W, \bigwedge^{d-1} T^{*} D\right)$ given by $\mathbf{t}^{\top}=\rho_{D}\left(\sigma^{\top}\right)$ is well defined for every $(d-1)$-dimensional submanifold $D$. However, the meaning of the integral of $\mathbf{t}(u)$, for a velocity field $u$ over $D$, depends on the orientation chosen for $D$. The orientation of $\partial \mathscr{R}$ is automatically determined by the requirements that $\mathscr{R}$ inherits its orientation from $\mathscr{M}$ and by the definition of the orientation of the boundary of a region. Writing the dependence of the induced surface force on the orientation assigned to an arbitrary surface, has been a standard practice in engineering mechanics analysis for a long time.

As a section of $L\left(W, \bigwedge^{d-1} T^{*} \mathscr{M}\right)$, a traction stress $\sigma$, is represented locally in the form $\left(x^{i}, \sigma_{1 \ldots \widehat{k} \ldots d r}\left(x^{j}\right)\right)$ or

$$
\sum_{k, r} \sigma_{1 \ldots \widehat{k} \ldots d r}\left(\mathrm{~d} x^{1} \wedge \cdots \wedge \widehat{\mathrm{d} x^{k}} \wedge \cdots \wedge \mathrm{d} x^{d}\right) \otimes g^{r} .
$$

The transposed, $\sigma^{\top}$, is represented by

$$
\sum_{k, r} \sigma_{1 \ldots \widehat{k} \ldots d r} g^{r} \otimes\left(\mathrm{d} x^{1} \wedge \cdots \wedge \widehat{\mathrm{d} x^{k}} \wedge \cdots \wedge \mathrm{d} x^{d}\right)
$$

and $\sigma(u)$ is represented locally by

$$
\sum_{k, r} \sigma_{1 \ldots \widehat{k} \ldots d r} u^{r} \mathrm{~d} x^{1} \wedge \cdots \wedge \widehat{\mathrm{d} x^{k}} \wedge \cdots \wedge \mathrm{d} x^{d} .
$$

I0.4. The Power in Terms of the Traction Stresses. For a traction stress field $\sigma$, the power of the induced surface force $\mathbf{t}_{\mathscr{R}}$ corresponding to a generalized velocity field $u$ on $\partial \mathscr{R}$ may be written now as

$$
\begin{aligned}
P=\int_{\partial \mathscr{R}} \mathbf{t}_{\mathscr{R}}(u) & =\int_{\partial \mathscr{R}} \rho_{\partial \mathscr{R}}(\sigma(u)), \\
& =\int_{\mathscr{R}} \mathrm{d}(\sigma(u)),
\end{aligned}
$$

so that

$$
F_{\mathscr{R}}(w)=\int_{\mathscr{R}} \mathbf{b}_{\mathscr{R}}(w)+\mathrm{d}(\sigma(w)) .
$$


Using the local representation of $\sigma(w)$ in (IO.28), the local expression for $\mathrm{d}(\sigma(w))$ ) is

$$
\begin{aligned}
\sum_{k, r}(-1)^{k-1} & \left(\sigma_{1 \ldots \widehat{k} \ldots d r} w^{r}\right)_{, k} \mathrm{~d} x^{1} \wedge \cdots \wedge \mathrm{d} x^{d} \\
= & \sum_{k, r}(-1)^{k-1}\left(\sigma_{1 \ldots \widehat{k} \ldots d r, k} w^{r}+\sigma_{1 \ldots \widehat{k} \ldots d r} w_{, k}^{r}\right) \mathrm{d} x^{1} \wedge \cdots \wedge \mathrm{d} x^{d}
\end{aligned}
$$

It is observed therefore that in the expression (IO.30) for the total power, the integrand depends linearly on both components of $w$ and their derivatives. In other words, the integrand is linear in the jet $j(w)$ of the the velocity field (see Section 5.7). In traditional continuum mechanics one obtains that the power of the external forces is equal to the power that the stresses perform on the derivatives of the velocity fields. In the general geometry of manifolds, one cannot disassociate the values of the derivatives of the vector field from the values of the vector field itself.

I0.5. Forces and Stresses for Kinematic Fluxes. Consider the case where the generalized velocity field represents the velocity field of material points in the physical space $\mathscr{S}$. For a configuration $\kappa: B \rightarrow \mathscr{S}$, the field $w$ is a section of the pullback of the tangent bundle $\kappa^{*}(T \mathscr{S})$ as in Example 5.7, and so $W=\kappa^{*}(T \mathscr{S})$. Hence, a traction stress is a section $\sigma$ of $L\left(\kappa^{*}(T \mathscr{S}), \bigwedge^{d-1} T^{*} B\right)$ and it is represented locally in the form

$$
\sum_{k, r} \sigma_{1 \ldots \widehat{k} \ldots d r}\left(\mathrm{~d} x^{1} \wedge \cdots \wedge \widehat{\mathrm{d} x^{k}} \wedge \cdots \wedge \mathrm{d} x^{d}\right) \otimes \mathrm{d} y^{r}
$$

Here, $\left\{\mathrm{d} y^{r}\right\}$ are the dual base vectors in $\left(\kappa^{*}(T \mathscr{S})\right)_{x}^{*} \cong T_{\kappa(x)}^{*} \mathscr{S}$ corresponding to the basis $\left\{\partial / \partial y^{r}\right\}$ of $\left(\kappa^{*}(T \mathscr{S})\right)_{x} \cong T_{\kappa(x)} \mathscr{S}$. Given a volume element $\theta$ on $B$ and the isomorphism $\iota_{\theta}: \bigwedge^{n-1}\left(T^{*} B\right) \longrightarrow T B$ as in (8.7), we may represent the stress $\sigma$ by $\iota_{\theta} \circ \sigma$, a section of $L\left(\kappa^{*}(T \mathscr{S}), T B\right)$. Using (8.6), $\iota_{\theta} \circ \sigma$ is represented locally by

$$
\sum_{k, r}(-1)^{k-1} \frac{\sigma_{1 \ldots \widehat{k} \ldots d r}}{\theta_{1 \ldots d}} \frac{\partial}{\partial x^{k}} \otimes \mathrm{d} y^{r},
$$

where $\theta_{1 \ldots d} \mathrm{~d} x^{1} \wedge \cdots \wedge \mathrm{d} x^{d}$ is the local representation of $\theta$.

As observed by Noll [Nol59], a reference configuration is a chart for the body manifold $B$. If the stress were a tensor, a section of $L\left(\kappa^{*}(T \mathscr{S}), T B\right)$, this would be reflected in its transformation rule under a change of chart. It is well known however that under a change of reference configuration (e.g., from the current configuration to some other reference configuration), the stress transforms using the Piola transform involving the Jacobian determinant. A volume element $\theta$ (e.g., the one induced by a reference configuration or the one induced by a mass density) enables the representation by a tensor. The last equation is analogous to the definition of the Kirchhoff stress, the counterpart of our $\sigma$, on the basis of the Cauchy stress tensor, corresponding to $\iota_{\theta} \circ \sigma$. 
I0.6. Force Fields and Traction Stresses for Kinetic Fluxes. This section is concerned with the situation where a generalized velocity field is a kinetic flux $J$ on $\mathscr{M}$ represented locally, as in Equation (7.9), in the form

$$
\sum_{k} J_{1 \ldots . . . . . d} \mathrm{~d} x^{1} \wedge \cdots \wedge \widehat{\mathrm{d} x^{k}} \wedge \cdots \wedge \mathrm{d} x^{d}
$$

This situation is relevant if one does not assume the existence of material points a priori, but obtains the kinetic flux from the balance of some extensive property as in Section 9. In addition, given a volume element $\theta$, a vector field $v$ induces a kinetic flux $v\lrcorner \theta$. For example, in the case of classical continuum mechanics, one may use the mass density as a volume element.

In light of Example 4.5, for the case of generalized velocities modeled by kinetic fluxes the action $\mathbf{b}(J)$ of a body force density $\mathbf{b}$ is represented in the form $\mathbf{b}(J)=$ $\alpha \wedge J$ for a unique I-form $\alpha$.

A traction stress will be a section of

$$
L\left(\bigwedge^{d-1} T^{*} \mathscr{M}, \bigwedge^{d-1} T^{*} \mathscr{M}\right)=\bigwedge^{d-1} T^{*} \mathscr{M} \otimes\left(\bigwedge^{d-1} T^{*} \mathscr{M}\right)^{*}
$$

From the discussion on multivectors, in particular Equation (4.28), a basis of $\left(\wedge^{d-1} T^{*} \mathscr{M}\right)^{*}$ which is dual to the basis $\left\{\mathrm{d} x^{1} \wedge \cdots \wedge \widehat{\mathrm{d} x^{r}} \wedge \cdots \wedge \mathrm{d} x^{d}\right\}_{r=1}^{d}$ is

$$
\left\{\frac{\partial}{\partial x^{1}} \wedge \cdots \wedge \frac{\widehat{\partial}}{\partial x^{r}} \wedge \cdots \wedge \frac{\partial}{\partial x^{d}}\right\}_{r=1}^{d}
$$

Hence, $\sigma$ is represented locally in the form

$$
\sum_{k, r} \sigma_{1 \ldots \widehat{k} \ldots d}^{1 \ldots . . ., d}\left(\mathrm{~d} x^{1} \wedge \cdots \wedge \widehat{\mathrm{d} x^{k}} \wedge \cdots \wedge \mathrm{d} x^{d}\right) \otimes\left(\frac{\partial}{\partial x^{1}} \wedge \cdots \wedge \frac{\widehat{\partial}}{\partial x^{r}} \wedge \cdots \wedge \frac{\partial}{\partial x^{d}}\right)
$$

and $\sigma(J)$ is represented locally as

$$
\sum_{k, r} \sigma_{1 \ldots \widehat{k} \ldots d}^{1 \ldots \widehat{r} \ldots d} J_{1 \ldots \widehat{r} \ldots d} \mathrm{~d} x^{1} \wedge \cdots \wedge \widehat{\mathrm{d} x^{k}} \wedge \cdots \wedge \mathrm{d} x^{d} .
$$

Let $\theta$ be a given volume element on $\mathscr{M}$. Then, $\theta$ induces an isomorphism

$$
L\left(\bigwedge^{d-1} T^{*} \mathscr{M}, \bigwedge^{d-1} T^{*} \mathscr{M}\right) \longrightarrow L(T \mathscr{M}, T \mathscr{M})
$$

given by

$$
\sigma \longmapsto \iota_{\theta} \circ \sigma \circ \iota_{\theta}^{-1} \text {. }
$$

Thus, a volume element enables the representation of a traction stress that is dual to kinetic-flux fields by a linear mapping $T \mathscr{M} \rightarrow T \mathscr{M}$. Let $\tilde{\sigma}=\iota_{\theta} \circ \sigma \circ \iota_{\theta}^{-1}$ be expressed locally as

$$
\sum_{k, r} \tilde{\sigma}_{\cdot r}^{k} \frac{\partial}{\partial x^{k}} \otimes \mathrm{d} x^{r}
$$


so that $\tilde{\sigma}_{\cdot r}^{k}=\mathrm{d} x^{k}\left(\tilde{\sigma}\left(\partial / \partial x^{r}\right)\right)$. Using (8.6) one has

$$
\begin{aligned}
& \tilde{\sigma}_{\cdot r}^{k}=\mathrm{d} x^{k}\left(\iota_{\theta} \circ \sigma \circ \iota_{\theta}^{-1}\left(\frac{\partial}{\partial x^{r}}\right)\right), \\
& =(-1)^{r-1} \theta_{1 \ldots d} \mathrm{~d} x^{k}\left(\iota_{\theta} \circ \sigma\left(\mathrm{d} x^{1} \wedge \cdots \wedge \widehat{\mathrm{d} x^{r}} \wedge \cdots \wedge \mathrm{d} x^{d}\right)\right), \\
& =(-1)^{r-1} \theta_{1 \ldots d} \mathrm{~d} x^{k} \iota_{\theta}\left(\sum_{l} \sigma_{1 \ldots \widehat{l} \ldots d}^{1 \ldots \widehat{r} \ldots, d} \mathrm{~d} x^{1} \wedge \cdots \wedge \widehat{\mathrm{d} x^{l}} \wedge \cdots \wedge \mathrm{d} x^{d}\right) \text {, } \\
& =(-1)^{r-1} \theta_{1 \ldots d} \sum_{l} \mathrm{~d} x^{k}\left(\frac{(-1)^{l-1}}{\theta_{1 \ldots d}} \sigma_{1 \ldots \widehat{l} \ldots d}^{1 \ldots . . . ., d} \frac{\partial}{\partial x^{l}}\right) \text {, } \\
& =(-1)^{r+k} \sigma_{1 \ldots \widehat{k} \ldots, d}^{1 \ldots . . ., d} .
\end{aligned}
$$

It follows that the isomorphism of Equation (IO.39) is actually independent of the choice of volume element. In other words, the equation above represents a natural isomorphism between $L\left(\bigwedge^{d-1} T^{*} \mathscr{M}, \bigwedge^{d-1} T^{*} \mathscr{M}\right)$ and $L(T \mathscr{M}, T \mathscr{M})=$ $T \mathscr{M} \otimes T^{*} \mathscr{M}$. An isomorphism

$$
\iota: T \mathscr{M} \otimes T^{*} \mathscr{M} \longrightarrow L\left(\bigwedge^{d-1} T^{*} \mathscr{M}, \bigwedge^{d-1} T^{*} \mathscr{M}\right)
$$

can be constructed without using provisionally a volume element as follows. Consider $\tilde{\sigma} \in T \mathscr{M} \otimes T^{*} \mathscr{M}$ represented by $\sum_{k, r} \tilde{\sigma}^{k}{ }_{r} \partial / \partial x^{k} \otimes \mathrm{d} x^{r}$. For any flux form $J$, represented as in (IO.34), set

$$
\left.\iota(\tilde{\sigma})(J)=\sum_{k, r} \tilde{\sigma}_{\cdot r}^{k} \frac{\partial}{\partial x^{k}}\right\lrcorner\left(\mathrm{d} x^{r} \wedge J\right) .
$$

Since the exterior product is bi-linear and the contraction is linear, $\iota(\tilde{\sigma})$ is indeed linear in $J$. To show that Equation (IO.42) is indeed a representation of $\iota$ as defined by (IO.44) we use the representation (IO.34) and obtain

$$
\begin{aligned}
\iota(\tilde{\sigma})(J) & \left.=\sum_{k, r} \tilde{\sigma}_{\cdot r}^{k} \frac{\partial}{\partial x^{k}}\right\lrcorner\left(\mathrm{d} x^{r} \wedge\left(\sum_{p} J_{1 \ldots . . . . . d} \mathrm{~d} x^{1} \wedge \cdots \wedge \widehat{\mathrm{d} x^{p}} \wedge \cdots \wedge \mathrm{d} x^{d}\right)\right), \\
& \left.=\sum_{k, r} \tilde{\sigma}_{\cdot r}^{k} \frac{\partial}{\partial x^{k}}\right\lrcorner\left((-1)^{r-1} J_{1 \ldots . . . . . d} \mathrm{~d} x^{1} \wedge \cdots \wedge \mathrm{d} x^{d}\right), \\
& =\sum_{k, r} \tilde{\sigma}_{\cdot r}^{k}(-1)^{r-1}(-1)^{k-1} J_{1 \ldots . . . . d} \mathrm{~d} x^{1} \wedge \cdots \wedge \widehat{\mathrm{d} x^{k}} \wedge \cdots \wedge \mathrm{d} x^{d}, \\
& =\sum_{k, r}(-1)^{r+k} \tilde{\sigma}_{\cdot r}^{k} J_{1 \ldots . . . . d} \mathrm{~d} x^{1} \wedge \ldots \wedge \widehat{\mathrm{d} x^{k}} \wedge \cdots \wedge \mathrm{d} x^{d} .
\end{aligned}
$$

Comparing the last equation with (IO.38), the result of Equation (IO.42) follows,

It seems to us quite intriguing that in spite of the various generalizations pertaining to the other variables, for the case under consideration, the traction stress is represented as a tensor in the traditional sense. 
I0.7. Cauchy's Theorem for Traction Stresses. In analogy with Section 7.4, we lay down the assumptions that will be used in the proof of Cauchy's theorem for traction stresses on manifolds. Then, we state the theorem and prove it.

We consider the collection $\left\{\mathbf{t}_{\mathscr{R}}\right\}$ for the various admissible regions $\mathscr{R} \subset \mathscr{M}$ so that each $\mathbf{t}_{\mathscr{R}}$ is a smooth section of $L\left(\left.W\right|_{\partial \mathscr{R}}, \bigwedge^{d-1}\left(T^{*} \partial \mathscr{R}\right)\right)$.

Assumption 1: Boundedness. There is a section $\xi$ of $L\left(J(W), \bigwedge^{d}\left(T^{*} \mathscr{M}\right)\right)$ such that for each $\mathscr{R}$,

$$
\left|\int_{\partial \mathscr{R}} \mathbf{t}_{\mathscr{R}}\left(\left.w\right|_{\partial \mathscr{R}}\right)\right| \leqslant \int_{\mathscr{R}}|\xi(j(w))|,
$$

for every smooth section of $W$. In the sequel we will use the notation $\mathbf{t}_{\mathscr{R}}(w)$ freely and will omit the indication of the restriction to the boundary.

Remark IO.I. In view of Section 5.7, a section $\xi$ of $L\left(J(W), \bigwedge^{d}\left(T^{*} \mathscr{M}\right)\right)$ is represented locally in the form

$$
\left(x^{i}\right) \longmapsto\left(x^{i}, \xi_{1 \ldots d p}\left(x^{i}\right), \Xi_{1 \ldots d q}^{j}\left(x^{i}\right)\right)
$$

so that the $n$-form $\xi(j(w))$ is represented locally as

$$
\sum_{p, j}\left(\xi_{1 \ldots d p} w^{p}+\Xi_{1 \ldots d p}^{j} w_{, j}^{p}\right) \mathrm{d} x^{1} \wedge \cdots \wedge \mathrm{d} x^{d} .
$$

Cauchy's postulate of locality for stresses is a straightforward analog of the one corresponding to fluxes.

Assumption 2: Cauchy's Postulate of Locality. Let $v_{1}, \ldots, v_{n-1} \in T_{x} \mathscr{M}, x \in \mathscr{M}$, be a collection of $n-1$ vectors. Let $\mathscr{R}$ be any region such that $x \in \partial \mathscr{R}, v_{1}, \ldots, v_{n-1} \in T_{x} \partial \mathscr{R}$, and the collection of vectors is positively oriented relative to the orientation of $\partial \mathscr{R}$. Then, $\mathbf{t}_{\mathscr{R}}^{\mathrm{T}}(x)\left(v_{1}, \ldots, v_{n-1}\right)$ is independent of $\mathscr{R}$.

Cauchy's postulate implies that for the given point $x \in \mathscr{M}, \mathbf{t}_{\mathscr{R}}(x)$ depends on $\mathscr{R}$ only through the tangent space $T_{x} \partial \mathscr{R}$ and the orientation induced on it. This makes it possible to define a mapping

$$
\Sigma_{x}:\left(T_{x} \mathscr{M}\right)^{d-1} \longrightarrow W_{x}^{*}
$$

such that for any collection $v_{1}, \ldots, v_{n-1} \in T_{x} \mathscr{M}$,

$$
\Sigma_{x}\left(v_{1}, \ldots, v_{d-1}\right)=\mathbf{t}_{\mathscr{R}}^{\top}(x)\left(v_{1}, \ldots, v_{d-1}\right)
$$

for any region $\mathscr{R}$ such that the collection $\left(v_{1}, \ldots, v_{d-1}\right) \in\left(T_{x} \partial \mathscr{R}\right)^{d-1}$ is positively oriented. Thus, the order in which the collection is written, specifies the orientation for which the action of $\Sigma_{x}$ applies. At this point, for an odd permutation $\pi$, there is no relation between $\Sigma_{x}\left(v_{1}, \ldots, v_{d-1}\right)$ and $\Sigma_{x}\left(v_{\pi_{1}}, \ldots, v_{\pi_{d-1}}\right)$. It is noted that $\Sigma_{x}\left(v_{1}, \ldots, v_{d-1}\right)$ depends only on the multi-vector $\mathfrak{v}=v_{1} \wedge \cdots \wedge v_{d-1}$. Thus, we have a fiber preserving mapping between vector bundles,

$$
\Sigma: T^{d-1} \mathscr{S} \longrightarrow W^{*}, \quad \text { such that }\left.\quad \Sigma\right|_{\left(T_{x} \mathscr{M}\right)^{d-1}}=\Sigma_{x} .
$$

The second locality postulate pertains to the body force. It is assumed that $\mathbf{b}_{\mathscr{R}}(x), x \in \mathscr{M}$ is independent of $\mathscr{R}$. Thus, we will simply use $\mathbf{b}$ for the body force 
in what follows. While this locality postulate is not needed in the proof of Cauchy's theorem for traction stresses, it will be used in our subsequent analysis.

Assumption 3: Regularity. The mapping $\Sigma$ is smooth.

We refer to the collection $\left\{\mathbf{t}_{\mathscr{R}}\right\}$ for the various regions $\mathscr{R}$ satisfying the foregoing assumptions as a system of Cauchy surface forces. These assumptions make it possible to prove Cauchy's theorem for traction stresses on manifolds, i.e., to prove that there exists a unique traction stress $\sigma$ such that (IO.24) and the equivalent (IO.25) hold.

Consider a generic point $x_{0} \in \mathscr{M}$ and a vector bundle chart $(\Phi, \varphi, U)$ with coordinates $\left(x^{i}, u^{p}\right), i=1, \ldots, d, p=1, \ldots, m$, in an open neighborhood $U$ of $x_{0}$. The local base vectors in $\pi^{-1}\{U\}$ will be denoted as $\left\{g_{p}\right\}$ so that the restriction of a section $w$ of $W$ to $U$ may be written in the form $\sum_{p} w^{p}\left(x^{i}\right) g_{p}$.

We want to prove that $\Sigma_{x_{0}}$ is multilinear and alternating, i.e., that $\mathbf{t}_{\mathscr{R}}^{\mathrm{T}}\left(x_{0}\right)$ is the restriction to $\left(T_{x_{0}} \partial \mathscr{R}\right)^{d-1}$ of a multilinear alternating mapping on $\left(T_{x_{0}} \mathscr{M}\right)^{d-1}$. With this objective in mind, we construct for each $p=1, \ldots, m$ a Cauchy flux system $\left\{\tau_{p \mathscr{R}}\right\}$ for which Cauchy's theorem for fluxes of Section 7.4 may be applied.

Let $\psi: U \rightarrow[0,1]$ be a smooth function having the following properties. (a) $\psi$ is compactly supported in $U$. (b) There is an open neighborhood $V$ of $x_{0}$ such that $V \subset U$ and for each $x \in V, \psi(x)=1$. Thus, $\psi$ decreases from $\mathrm{I}$ to $\mathrm{O}$ in the subset of $U$ outside $V$. For each $p=1, \ldots, m$, we define the section $w_{(p)}$ of $W$ by

$$
w_{(p)}(x)=\psi(x) g_{p} \quad \text { for } x \in U, \quad \text { and } \quad w_{(p)}(x)=0 \quad \text { for } x \notin U .
$$

By the definition of the cutoff function $\psi, w_{(p)}$ is evidently a smooth field. Letting $\tilde{\psi}$ be the local representative of $\psi$ in $U$, then, locally, $w_{(p)}^{l}\left(x^{i}\right)=\tilde{\psi}\left(x^{i}\right) \delta_{p}^{l}, w_{(p), j}^{l}=$ $\tilde{\psi}_{, j} \delta_{p}^{l}$.

For each region $\mathscr{R}$ and $p=1, \ldots, m$, consider the flux distribution $\tau_{p \mathscr{R}}$ defined by

$$
\tau_{p \mathscr{R}}=\mathbf{t}_{\mathscr{R}}\left(w_{(p)}\right),
$$

so that

$$
\begin{aligned}
\tau_{p \mathscr{R}}(x)\left(v_{1}, \ldots, v_{d-1}\right) & =\mathbf{t}_{\mathscr{R}}(x)\left(w_{(p)}(x)\right)\left(v_{1}, \ldots, v_{d-1}\right), \\
& =\Sigma_{x}\left(v_{1}, \ldots, v_{d-1}\right)\left(w_{(p)}(x)\right) .
\end{aligned}
$$


To show that $\left\{\tau_{p \mathscr{R}}\right\}$ satisfy the boundedness condition of Section 7.4, we observe that

$$
\begin{aligned}
\left|\int_{\partial \mathscr{R}} \tau_{p \mathscr{R}}\right| & =\left|\int_{\partial \mathscr{R}} \mathbf{t}_{\mathscr{R}}\left(w_{(p)}\right)\right|, \\
& \leqslant \int_{\mathscr{R}} \mid \xi\left(j\left(w_{(p)}\right) \mid,\right. \\
& =\int_{\mathscr{R} \cap U} \mid \xi\left(j\left(w_{(p)}\right) \mid,\right. \\
& =\int_{\varphi(\mathscr{R} \cap U)}\left|\xi_{1 \ldots d p} \tilde{\psi}+\Xi_{1 \ldots d p}^{j} \tilde{\psi}_{, j}\right| \mathrm{d} x^{1} \cdots \mathrm{d} x^{d}, \\
& \leqslant \int_{\varphi(\mathscr{R} \cap U)} C\left(\left|\xi_{1 \ldots d p}\right|+\sum_{j}\left|\Xi_{1 \ldots d p}^{j}\right|\right) \mathrm{d} x^{1} \cdots \mathrm{d} x^{d}, \\
& \leqslant \int_{\varphi(\mathscr{R} \cap U)} \varsigma(p) 1 \ldots d \\
& =\int_{\mathscr{R}} \varsigma_{(p)},
\end{aligned}
$$

where in the fifth line, the constant $C$ exists because $\tilde{\psi}_{, i}$ is different from zero in a compact set and and $\max _{x} \psi(x)=1$. In the sixth line,

$$
\varsigma(p) 1 \ldots d\left(x^{i}\right)=C\left(\left|\xi_{1 \ldots d p}\left(x^{i}\right)\right|+\sum_{j}\left|\Xi_{1 \ldots d p}^{j}\left(x^{i}\right)\right|\right), \quad x \in U ;
$$

$\varsigma_{(p)}$ is the compactly supported $d$-form on $\mathscr{M}$ such that $\varsigma(x)$ is represented by $\varsigma(p) 1 \ldots d \mathrm{~d} x^{1} \wedge \cdots \wedge \mathrm{d} x^{d}$ for $x \in U$ and $\varsigma(x)=0$ for $x \notin U$.

The flux system $\left\{\tau_{p \mathscr{R}}\right\}$ satisfies the locality assumption because

$$
\begin{aligned}
\tau_{p \mathscr{R}}(x)\left(v_{1}, \ldots, v_{d-1}\right) & =\mathbf{t}_{\mathscr{R}}(x)\left(w_{(p)}\right)\left(v_{1}, \ldots, v_{d-1}\right), \\
& =\mathbf{t}_{\mathscr{R}}^{\mathrm{T}}(x)\left(v_{1}, \ldots, v_{d-1}\right)\left(w_{(p)}\right),
\end{aligned}
$$

which by the locality assumption for surface forces is independent of $\mathscr{R}$ as long as $\left(v_{1}, \ldots, v_{d-1}\right)$ is a positively oriented collection of vectors tangent to $\partial \mathscr{R}$.

Let $\mathfrak{t}_{p}: T^{d-1} \mathscr{M} \longrightarrow \mathbb{R}$ be the Cauchy-Whitney mapping corresponding to the flux system $\left\{\tau_{p \mathscr{R}}\right\}$. We have to show that $\mathfrak{t}_{p}$ is smooth. Independently of the region $\mathscr{R}$ to which the collection of tangent vectors is positively oriented, one has

$$
\begin{aligned}
\mathfrak{t}_{p}\left(x, v_{1}, \ldots, v_{d-1}\right) & =\tau_{p \mathscr{R}}(x)\left(v_{1}, \ldots, v_{d-1}\right), \\
& =\mathbf{t}_{\mathscr{R}}^{\top}(x)\left(v_{1}, \ldots, v_{d-1}\right)\left(w_{(p)}(x)\right), \\
& =\Sigma\left(x, v_{1}, \ldots, v_{d-1}\right)\left(w_{(p)}(x)\right) .
\end{aligned}
$$

Since $\Sigma$ is assumed to be smooth and $w_{(p)}$ is smooth by its construction, so is $\mathfrak{t}_{p}$.

Cauchy's theorem for fluxes implies now that for each $p$ there is a flux form $\sigma_{p} \in \Omega^{d-1}(\mathscr{M})$ such that $\tau_{p \mathscr{R}}\left(x_{0}\right)=\rho_{\partial \mathscr{R}}\left(\sigma_{p}\left(x_{0}\right)\right)$. Using the definition (IO.53),

$$
\begin{aligned}
\mathbf{t}_{\mathscr{R}}\left(x_{0}\right)\left(g_{p}\right) & =\mathbf{t}_{\mathscr{R}}\left(x_{0}\right)\left(w_{(p)}\left(x_{0}\right)\right), \\
& =\rho_{\partial \mathscr{R}}\left(\sigma_{p}\left(x_{0}\right)\right),
\end{aligned}
$$


and it follows that

$$
\begin{aligned}
\mathbf{t}_{\mathscr{R}}\left(x_{0}\right)\left(w_{0}\right) & =\mathbf{t}_{\mathscr{R}}\left(x_{0}\right)\left(\sum_{p} w_{0}^{p} g_{p}\right), \\
& =\sum_{p} w_{0}^{p} \mathbf{t}_{\mathscr{R}}\left(x_{0}\right)\left(g_{p}\right), \\
& =\sum_{p} w_{0}^{p} \rho_{\partial \mathscr{R}}\left(\sigma_{p}\left(x_{0}\right)\right) .
\end{aligned}
$$

Defining the section $\sigma$ of $L\left(W, \bigwedge^{d-1}\left(T^{*} \mathscr{M}\right)\right)$ by

$$
\sigma(w)=\sum_{p} w^{p} \sigma_{p}
$$

we obtain

$$
\begin{aligned}
\rho_{\partial \mathscr{R}}\left(\sigma^{\top}\left(x_{0}\right)\right)\left(v_{1}, \ldots, v_{d-1}\right)\left(w_{0}\right) & =\sigma\left(x_{0}\right)\left(w_{0}\right)\left(T \iota_{\partial \mathscr{R}}\left(v_{1}\right), \ldots, T \iota_{\partial \mathscr{R}}\left(v_{d-1}\right)\right), \\
& =\sum_{p} w_{0}^{p} \rho_{\partial \mathscr{R}}\left(\sigma_{p}\left(x_{0}\right)\right)\left(v_{1}, \ldots, v_{d-1}\right), \\
& =\mathbf{t}_{\mathscr{R}}^{\top}\left(x_{0}\right)\left(v_{1}, \ldots, v_{d-1}\right)\left(w_{0}\right),
\end{aligned}
$$

which is the required Cauchy formula (IO.24).

IO.8. Variational Stresses. Continuing the observations made in Section IO.4, we conclude that the total power of the body force and surface force for a region $\mathscr{R}$ is given by

$$
F_{\mathscr{R}}(w)=\int_{\mathscr{R}} \mathbf{b}(w)+\mathrm{d}(\sigma(w)) .
$$

The local expression for the integrand is

$$
\sum_{k, r}\left[\left(\mathbf{b}_{1 \ldots d r}+(-1)^{k-1} \sigma_{1 \ldots \widehat{k} \ldots d r, k}\right) w^{r}+(-1)^{k-1} \sigma_{1 \ldots \widehat{k} \ldots d r} w_{, k}^{r}\right] \mathrm{d} x^{1} \wedge \cdots \wedge \mathrm{d} x^{d} .
$$

It is noted that the values of the $d$-form in the expression above are linear in the local representatives $\left(w^{k}, w_{, i}^{l}\right)$ ( $c f$. Section 5.7) of $j(w)$. Thus, there is a section $S$ of $L\left(J(W), \bigwedge^{d}\left(T^{*} \mathscr{M}\right)\right)$ such that $\mathbf{b}(w)+\mathrm{d}(\sigma(w))=S(j(w))$. We will refer to $S$ as the variational stress. This may be summarized by

$$
F_{\mathscr{R}}(w)=\int_{\mathscr{R}} \mathbf{b}(w)+\mathrm{d}(\sigma(w))=\int_{\mathscr{R}} \mathbf{b}(w)+\int_{\partial \mathscr{R}} \mathbf{t}_{\mathscr{R}}(w)=\int_{\mathscr{R}} S(j(w)) .
$$

which is the metric independent version of the principle of virtual power. In contrast with the classical version of the principle of virtual work, for the metric independent analysis one has to make a distinction between the traction stress $\sigma$ that induces the surface traction using the generalized Cauchy formula and the variational stress which determines the density of the power via the principle of virtual power. 
Observing Remark IO.I, $S$ is represented locally in the form $\left(x^{i}, R_{1 \ldots d p}, S_{1 \ldots d p}^{k}\right)$ or equivalently

$$
\left(\sum_{p} R_{1 \ldots d p}\left(\mathrm{~d} x^{1} \wedge \cdots \wedge \mathrm{d} x^{d}\right) \otimes g^{p}, \sum_{k, p} S_{1 \ldots d p}^{k}\left(\mathrm{~d} x^{1} \wedge \cdots \wedge \mathrm{d} x^{d}\right) \otimes \frac{\partial}{\partial x^{k}} \otimes g^{p}\right)
$$

so that $S(j(w))$ is represented locally by

$$
\left(\sum_{p} R_{1 \ldots d p} w^{p}+\sum_{k, p} S_{1 \ldots d p}^{k} w_{, k}^{p}\right) \mathrm{d} x^{1} \wedge \cdots \wedge \mathrm{d} x^{d} .
$$

Since the principle of virtual power holds for any region $\mathscr{R}$ and virtual velocity field $w$, it follows from the smoothness of the various fields and the local representation of (IO.64) and (IO.67) that

$$
\begin{aligned}
R_{1 \ldots d r} & =\mathbf{b}_{1 \ldots d r}+\sum_{k}(-1)^{k-1} \sigma_{1 \ldots \widehat{k} \ldots d r, k}, \\
S_{1 \ldots d r}^{k} & =(-1)^{k-1} \sigma_{1 \ldots \widehat{k} \ldots d r} .
\end{aligned}
$$

Using the second equation above, the first equation may be written in the form

$$
\mathbf{b}_{1 \ldots d r}=R_{1 \ldots d r}-\sum_{k} S_{1 \ldots d r, k}^{k} .
$$

As an element of the space of sections of $L\left(J(W), \bigwedge^{d} T^{*} \mathscr{M}\right)$, a variational stress $S$ induces the form $S^{\top} \in \Omega^{d}\left(T^{*} \mathscr{M}, J(W)^{*}\right)$ which assumes values in the dual of the jet bundle and is defined by

$$
S^{\top}\left(v_{1}, \ldots, v_{d}\right)(E)=S(E)\left(v_{1}, \ldots, v_{d}\right) .
$$

It is noted that for a velocity field $w$, the components $w_{, k}^{r}$ of the jet $j(w)$ do not represent an invariant geometrical object unless they are in conjunction with the components $w^{i}$. On the other hand, the second of Equations (I0.68) and the fact that the traction stress is an invariant object, imply that the dual components $S_{1 \ldots d r}^{k}$ do represent an invariant object which we describe below. In the terminology of [Pal68], the components $S_{1 \ldots, d r}^{k}$ represent the symbol of $S$, where $S$ is regarded as a linear differential operator.

To present the relations without recourse to local representation we construct a surjective vector bundle morphism

$$
p_{\sigma}: L\left(J(W), \bigwedge^{d} T^{*} \mathscr{M}\right) \longrightarrow L\left(W, \bigwedge^{d-1} T^{*} \mathscr{M}\right) .
$$

that associates with every variational stress $S$ a traction stress $\sigma=p_{\sigma} \circ S$ which we also write as $p_{\sigma}(S)$.

Using the linear jet projection mapping $\pi^{1}: J(W) \rightarrow W$, consider the vertical sub-bundle $V J(W)=\operatorname{Kernel} \pi^{1}$. Since $\pi^{1}$ is represented locally by $\left(x^{i}, u^{p}, A_{k}^{q}\right) \mapsto$ $\left(x^{i}, u^{p}\right)$, an element of $V J(W)$ is represented locally in the form $\left(x^{i}, 0, A_{k}^{q}\right)$. In other words, the fiber $V J(W)_{x_{0}}$ contains jets of sections of $W$ that vanish at $x_{0}$. It 
is noted that there is a natural vector bundle isomorphism $V J(W) \cong L(T \mathscr{M}, W)$ by which an element of $V J(W)$ is represented in the form $\left(x^{i}, A_{k}^{q}\right)$. We use $\iota_{V}$ : $V J(W) \hookrightarrow J(W)$ to denote the inclusion of the vertical sub-bundle-a vector bundle morphism over $\mathscr{M}$ represented by $\left(x^{i}, A_{k}^{q}\right) \mapsto\left(x^{i}, 0, A_{k}^{q}\right)$. Then, the dual vector bundle morphism $\iota_{V}^{*}: J(W)^{*} \rightarrow(V J(W))^{*} \cong L(W, T \mathscr{M})$ is a projection represented locally in the form $\left(x^{i}, \xi_{p}, \Xi_{q}^{i}\right) \mapsto\left(x^{i}, \Xi_{q}^{i}\right)$-the restriction of $\xi \in J(W)^{*}$ to vertical elements of the jet bundle. Thus, $\iota_{V}^{*}(\xi)(A), A \in V J(W)$, is represented by $\sum_{i, q} \Xi_{q}^{i} A_{i}^{q}$. Similarly, for a section $S$ of $L\left(J(W), \bigwedge^{d} T^{*} \mathscr{M}\right), \iota_{V}^{*}(S)$, a section of $L\left(V J(W), \bigwedge^{d} T^{*} \mathscr{M}\right)$, is given by $\iota_{V}^{*}(S)(x)(A)=S(x)\left(\iota_{V}(A)\right) \in \bigwedge^{d} T_{x}^{*} \mathscr{M}$. The evaluation $\iota_{V}^{*}(S)(x)(A)$ is represented by $\sum_{k, p} S_{1 \ldots d p}^{k}(x) A_{, k}^{p} \mathrm{~d} x^{1} \wedge \cdots \wedge \mathrm{d} x^{d}$ and so $\iota_{V}^{*}(S)$ is represented in the form

$$
\sum_{k, p} S_{1 \ldots d p}^{k}\left(\mathrm{~d} x^{1} \wedge \cdots \wedge \mathrm{d} x^{d}\right) \otimes \frac{\partial}{\partial x^{k}} \otimes g^{p} .
$$

As mentioned above, the object $\iota_{V}^{*}(S)$ is the symbol of the linear differential operator $S$ as defined in [Pal68].

Using the isomorphism $V J(W) \cong L(T \mathscr{M}, W)$, we regard $\iota_{V}^{*}(S)$ as a section of

$$
\begin{aligned}
L\left(L(T \mathscr{M}, W), \bigwedge^{d} T^{*} \mathscr{M}\right) & \cong\left(\bigwedge^{d} T^{*} \mathscr{M}\right) \otimes L(T \mathscr{M}, W)^{*}, \\
& \cong\left(\bigwedge^{d} T^{*} \mathscr{M}\right) \otimes L(W, T \mathscr{M}), \\
& \cong\left(\bigwedge^{d} T^{*} \mathscr{M}\right) \otimes T \mathscr{M} \otimes W^{*} .
\end{aligned}
$$

It follows that a section of $\bigwedge^{n}\left(T^{*} \mathscr{M}, L(W, T \mathscr{M})\right)$ may be represented locally in the form $\sum_{a} \theta \otimes v_{a} \otimes \varphi^{a}$ for a $d$-form $\theta$ and pairs $v_{a}, \varphi^{a}$ of sections of $T \mathscr{M}$ and $W^{*}$, respectively. We can use the contraction of the second and first factors in the product to obtain $\left.\sum_{a}\left(v_{a}\right\lrcorner \theta\right) \otimes \varphi^{a}$. Thus, we have a natural mapping

$$
\begin{aligned}
C: L\left(L(T \mathscr{M}, W), \bigwedge^{d} T^{*} \mathscr{M}\right) & \longrightarrow \bigwedge^{d-1}\left(T^{*} \mathscr{M}\right) \otimes W^{*} \\
& \cong L\left(W, \bigwedge^{d-1} T^{*} \mathscr{M}\right)
\end{aligned}
$$

It follows that

$$
p_{\sigma}:=\mathrm{C} \circ \iota_{V}^{*}: L\left(L(T \mathscr{M}, W), \bigwedge^{d} T^{*} \mathscr{M}\right) \longrightarrow L\left(W, \bigwedge^{d-1} T^{*} \mathscr{M}\right)
$$

associates a traction stress $\sigma=p_{\sigma} \circ S$ with any variational stress $S$. Let $S$ be a variational stress. Using (IO.72) and Section 4.9, C is represented locally by

$$
\begin{aligned}
\sum_{k, p} S_{1 \ldots d p}^{k}\left(\mathrm{~d} x^{1}\right. & \left.\left.\wedge \cdots \wedge \mathrm{d} x^{d}\right) \otimes \frac{\partial}{\partial x^{k}} \otimes g^{p} \longmapsto \sum_{k, p} S_{1 \ldots d p}^{k} \frac{\partial}{\partial x^{k}}\right\lrcorner\left(\mathrm{d} x^{1} \wedge \cdots \wedge \mathrm{d} x^{d}\right) \otimes g^{p} \\
& =\sum_{k, p}(-1)^{k-1} S_{1 \ldots d p}^{k}\left(\mathrm{~d} x^{1} \wedge \cdots \wedge \widehat{\mathrm{d} x^{k}} \wedge \cdots \wedge \mathrm{d} x^{d}\right) \otimes g^{p} . \quad \text { (IO.76) }
\end{aligned}
$$


We conclude that in case $\sigma=p_{\sigma}(S)$, then, $\sigma_{1 \ldots \widehat{k} \ldots d p}=(-1)^{k-1} S_{1 \ldots d p}^{k}$ as expected.

I0.9. The Divergence of a Variational Stress. We have seen in the previous section that a given variational stress $S$ induces a unique traction stress field $\sigma=$ $p_{\sigma}(S)$ which in turn, induces a surface force field on the boundary of each region using the Cauchy formula (IO.24). In this section we define a generalization of the divergence operator of classical vector analysis that may be applied to a variational stress field to give the body force field. As indicated in the first of (IO.68) and again below, the traction stress field does not contain, in the general geometric setting considered here, all the information needed in order to calculate the body force.

The divergence, $\operatorname{div} S$, of the variational stress field $S$ is a section of $L\left(W, \bigwedge^{d}\left(T^{*} \mathscr{M}\right)\right)$ which is defined invariantly by

$$
\operatorname{div} S(w)=\mathrm{d}\left(p_{\sigma}(S)(w)\right)-S\left(j^{1}(w)\right),
$$

for every differentiable vector field $w$. To present the local expression for $\operatorname{div} S$ we first note that if $\sigma=p_{\sigma}(S)$, then $\mathrm{d}(\sigma(w))$ is represented locally by

$$
\begin{aligned}
\sum_{k, p} \mathrm{~d}\left(\sigma_{1 \ldots \widehat{k} \ldots d p} w^{p}\right) \wedge \mathrm{d} x^{1} \wedge \cdots \wedge \widehat{\mathrm{d} x^{k}} \wedge \cdots \wedge \mathrm{d} x^{d} \\
=\sum_{k, p}\left(\sigma_{1 \ldots \widehat{k} \ldots d p} w^{p}\right)_{, k} \mathrm{~d} x^{k} \wedge \mathrm{d} x^{1} \wedge \cdots \wedge \widehat{\mathrm{d} x^{k}} \wedge \cdots \wedge \mathrm{d} x^{d}, \\
=\sum_{k, p}\left(\sigma_{1 \ldots \widehat{p} \ldots d p} w^{p}\right)_{, k}(-1)^{k-1} \mathrm{~d} x^{1} \wedge \cdots \wedge \mathrm{d} x^{d}, \\
=\sum_{k, p}\left(S_{1 \ldots d p}^{k} w^{p}\right)_{, k} \mathrm{~d} x^{1} \wedge \cdots \wedge \mathrm{d} x^{d} .
\end{aligned}
$$

Using Equation (I0.67), the local expression for $\operatorname{div} S(w)$ is therefore

$$
\begin{array}{r}
\sum_{k, p}\left[\left(S_{1 \ldots d p}^{k} w^{p}\right)_{, k}-\left(\sum_{p} R_{1 \ldots d p} w^{p}+\sum_{k, p} S_{1 \ldots d p}^{k} w_{, k}^{p}\right)\right] \mathrm{d} x^{1} \wedge \cdots \wedge \mathrm{d} x^{d} \\
=\sum_{k, p}\left(S_{1 \ldots d p, k}^{k}-R_{1 \ldots d p}\right) w^{p} \mathrm{~d} x^{1} \wedge \cdots \wedge \mathrm{d} x^{d}
\end{array}
$$

so that $\operatorname{div} S$ is represented locally by

$$
\sum_{k, p}\left(S_{1 \ldots d p, k}^{k}-R_{1 \ldots d p}\right) \mathrm{d} x^{1} \wedge \cdots \wedge \mathrm{d} x^{d} \otimes g^{p} .
$$

It is noted that in the case where $R_{1 \ldots d p}=0$ locally, the expression for the divergence reduces to the traditional expression for the divergence of a tensor field in a Euclidean space.

Given a variational stress $S$, Equation (I0.69) implies that

$$
\mathbf{b}=-\operatorname{div} S
$$


is the body force that satisfies the principle of virtual power (IO.65) - an obvious generalization of the differential equation for the stress tensor in continuum mechanics.

\section{iI. EXample: Metric InVariant Electromagnetism}

The formulation of electromagnetism in spacetime $\mathscr{E}$ may serve as an example for the simplicity, even elegance, resulting from the use of the theory of differentiable manifolds and integration of differential forms. In addition, it enables one to formulate some aspects of electromagnetism independently of a Riemannian metric. The first versions of electromagnetic theory that do not use a Riemannian metric appeared early in the I900's. Whittaker [Whi53, pp. 192-196], attributes the first work in this direction to Kottler, I922. He wrote:

"... seeking to abolish the privileged position of geometry in physics, and indeed inquiring how far it may be possible to construct a physics independent of geometry. Since the notion of metric is a complicated one, which requires measurements with clocks and scales, generally with rigid bodies, which themselves are systems of great complexity, it seems undesirable to take metric as fundamental, particularly for phenomena which are simpler and actually independent of it."

In [TT60, Section F] Truesdell and Toupin adopt the same point of view and write

"This "metrical independence" of the conservation laws has been noted by Van Dantzig [1934]. As he remarks, the concept of metric and the measurement of lengths, angles, and time intervals is perhaps one of the most sophisticated and complex aspects of any physical theory. Furthermore, is not the intuitive notion of conservation of charge, for example, quite independent of measurements of length and time? We view the conservation of charge and magnetic flux as independent of ideas like inertial frames, rigid rods, absolute or uniform time, Lorentz transformations, Galilean transformations, etc., and hence as deserving an independent mathematical expression.'

This approach to electromagnetism is referred to as premetric electrodynamics in recent work. (See for example [HIOo6] and works cited therein.) Here, we follow the approach of [SegO2]. It is noted that representing the body as a general differentiable manifold and formulating the theory without using a metric, enables one to view the Lagrangian and Eulerian versions of any one of the various electromagnetic fields defined in [DOO5, DOo9] as two representations of one geometrical object. In addition, the framework presented here is independent of constitutive relations such as the aether relations.

In the following, we will use the notation scheme of Section 9. 
II.I. Charge and Its Conservation. The first fundamental quantity of electromagnetism that we consider is the 3 -form $\mathfrak{J}$ in $\mathscr{E}$ modeling the 4 -current density. The existence of $\mathfrak{J}$ may be motivated by the theory of fluxes outlined in Section 7.4. In frame coordinates we may use Equation (9.6) and write locally

$$
\begin{aligned}
\mathfrak{J}= & \sum_{k} \mathfrak{J}_{1 \ldots \widehat{k} \ldots 4} \mathrm{~d} z^{1} \wedge \cdots \wedge \widehat{\mathrm{d} z^{k}} \wedge \cdots \wedge \mathrm{d} z^{4} \\
= & \rho_{123} \mathrm{~d} z^{2} \wedge \mathrm{d} z^{3} \wedge \mathrm{d} z^{4}-J_{23} \mathrm{~d} z^{1} \wedge \mathrm{d} z^{3} \wedge \mathrm{d} z^{4} \\
& \quad-J_{13} \mathrm{~d} z^{1} \wedge \mathrm{d} z^{2} \wedge \mathrm{d} z^{4}-J_{12} \mathrm{~d} z^{1} \wedge \mathrm{d} z^{2} \wedge \mathrm{d} z^{3},
\end{aligned}
$$

where $\rho=\rho_{123} \mathrm{~d} x^{1} \wedge \mathrm{d} x^{2} \wedge \mathrm{d} x^{3}$ is the space charge density and

$$
J=\sum_{i} J_{1 . \ldots \hat{\ldots}} \mathrm{d} x^{1} \wedge \cdots \wedge \widehat{\mathrm{d} x^{i}} \wedge \cdots \wedge \mathrm{d} x^{3}
$$

is the 2 -form in space describing the current density.

In case a volume element $\theta=\vartheta \mathrm{d} z^{1} \wedge \cdots \wedge \mathrm{d} z^{4}$ is given on spacetime and a volume element $\theta_{0}=\vartheta_{0} \mathrm{~d} x^{1} \wedge \mathrm{d} x^{2} \wedge \mathrm{d} x^{3}$ is given in $\mathscr{S}$ one may use the isomorphism $\iota_{\theta}$ defined in Section 8.3 to represent the flux forms by tangent vectors $\mathbf{j}=\sum \mathbf{j}^{i} \partial / \partial x^{i}$ and $w=\sum w^{k} \partial / \partial z^{k}$ such that $\left.\mathfrak{J}=w\right\lrcorner \theta$ and $\left.J=\mathbf{j}\right\lrcorner \theta_{0}$. It follows from Equation (8.6) that

$$
\mathbf{j}^{i}=(-1)^{i-1} \frac{J_{1 \ldots \widehat{l} \ldots 3}}{\vartheta_{0}}, \quad w^{k}=(-1)^{k-1} \frac{\mathfrak{J}_{1 \ldots \widehat{k} \ldots 4}}{\vartheta} .
$$

Using Equation (9.7) for $k>1$,

$$
\mathfrak{J}_{1 \ldots \widehat{k} \ldots 4}=(-1)^{k-1} w^{k} \vartheta=-J_{1 \ldots \widehat{k-1} \ldots 3}=-(-1)^{k-2} \mathbf{j}^{k-1} \vartheta_{0},
$$

and so

$$
w^{1}=\frac{\rho_{123}}{\vartheta}, \quad w^{2}=\frac{\vartheta_{0}}{\vartheta} \mathbf{j}^{1}, \quad w^{3}=\frac{\vartheta_{0}}{\vartheta} \mathbf{j}^{2}, \quad w^{4}=\frac{\vartheta_{0}}{\vartheta} \mathbf{j}^{3}
$$

(cf. [MTW73, p. 8I and p. II3], [LL95, pp. 60-7I]).

A basic assumption of electromagnetism is conservation of charge, i.e., for each region $\mathfrak{R}$ of spacetime

$$
\int_{\partial \mathfrak{R}} \mathfrak{J}=0 .
$$

Thus, it follows from Stokes's theorem that $\mathrm{d} \mathfrak{J}=0$.

The Maxwell 2 -form $\mathfrak{g}$ is a flow potential for the flux so that $\mathfrak{J}=\mathrm{d} \mathfrak{g}$. The local representation of the Maxwell form in Lorentzian frame coordinates is given by

$$
\begin{aligned}
\mathfrak{g}= & \sum_{k<l} \mathfrak{g}_{k l} \mathrm{~d} z^{k} \wedge \mathrm{d} z^{l}, \\
= & H_{1} \mathrm{~d} z^{1} \wedge \mathrm{d} z^{2}+H_{2} \mathrm{~d} z^{1} \wedge \mathrm{d} z^{3}+H_{3} \mathrm{~d} z^{1} \wedge \mathrm{d} z^{4} \\
& \quad+D_{3} \mathrm{~d} z^{2} \wedge \mathrm{d} z^{3}-D_{2} \mathrm{~d} z^{2} \wedge \mathrm{d} z^{4}+D_{1} \mathrm{~d} z^{3} \wedge \mathrm{d} z^{4}
\end{aligned}
$$


where $D_{i}$ are the component of the electric displacement and $H_{i}$ are the components of the magnetic field intensity. The matrix representing $\mathfrak{g}$ is therefore

$$
\left(\begin{array}{cccc}
0 & H_{1} & H_{2} & H_{3} \\
-H_{1} & 0 & D_{3} & -D_{2} \\
-H_{2} & -D_{3} & 0 & D_{1} \\
-H_{3} & D_{2} & -D_{1} & 0
\end{array}\right) .
$$

The components of the equation $\mathfrak{J}=\mathrm{d} \mathfrak{g}$ are

$$
\begin{aligned}
-\mathbf{j}^{3} \vartheta_{0} & =-J_{12}=\mathfrak{J}_{123}=\mathfrak{g}_{12,3}-\mathfrak{g}_{13,2}+\mathfrak{g}_{23,1}=\frac{\partial H_{1}}{\partial x^{2}}-\frac{\partial H_{2}}{\partial x^{1}}+\frac{\partial D_{3}}{\partial t}, \\
-\mathbf{j}^{1} \vartheta_{0} & =-J_{23}=\mathfrak{J}_{134}=\mathfrak{g}_{13,4}-\mathfrak{g}_{14,3}+\mathfrak{g}_{34,1}=\frac{\partial H_{2}}{\partial x^{3}}-\frac{\partial H_{3}}{\partial x^{2}}+\frac{\partial D_{1}}{\partial t}, \\
\mathfrak{j}^{2} \vartheta_{0} & =-J_{13}=\mathfrak{J}_{124}=\mathfrak{g}_{12,4}-\mathfrak{g}_{14,2}+\mathfrak{g}_{24,1}=\frac{\partial H_{1}}{\partial x^{3}}-\frac{\partial H_{3}}{\partial x^{1}}-\frac{\partial D_{2}}{\partial t}, \\
\rho_{1 \ldots 4} & =\mathfrak{J}_{234}=\mathfrak{g}_{23,4}-\mathfrak{g}_{24,3}+\mathfrak{g}_{34,2}=\frac{\partial D_{3}}{\partial x^{3}}+\frac{\partial D_{2}}{\partial x^{2}}+\frac{\partial D_{1}}{\partial x^{1}}
\end{aligned}
$$

- the first part of Maxwell's equations.

II.2. The Faraday 2-Form. The second fundamental object of electromagnetism is the Faraday 2 -form $\mathfrak{f}$. Using frame coordinates, $\mathfrak{f}$ is represented locally by

$$
\begin{aligned}
\mathfrak{f}= & \sum_{k<l} \mathfrak{f}_{k l} \mathrm{~d} z^{k} \wedge \mathrm{d} z^{l} \\
=- & E_{1} \mathrm{~d} z^{1} \wedge \mathrm{d} z^{2}-E_{2} \mathrm{~d} z^{1} \wedge \mathrm{d} z^{3}-E_{3} \mathrm{~d} z^{1} \wedge \mathrm{d} z^{4} \\
& \quad+B_{3} \mathrm{~d} z^{2} \wedge \mathrm{d} z^{3}-B_{2} \mathrm{~d} z^{2} \wedge \mathrm{d} z^{4}+B_{1} \mathrm{~d} z^{3} \wedge \mathrm{d} z^{4}
\end{aligned}
$$

where $E_{i}$ are the components of the electric field in space and $B_{i}$ are the components of the magnetic flux density. Thus, the matrix representation of $\mathfrak{f}$ is

$$
\left(\begin{array}{cccc}
0 & -E_{1} & -E_{2} & -E_{3} \\
E_{1} & 0 & B_{3} & -B_{2} \\
E_{2} & -B_{3} & 0 & B_{1} \\
E_{3} & B_{2} & -B_{1} & 0
\end{array}\right) .
$$

The other set of Maxwell's equations state that the Faraday 2 -form is closed, i.e., $\mathrm{d} \mathfrak{f}=0$. The local expression in frame components is

$$
\begin{aligned}
0= & \left(-E_{1,3}+E_{2,2}+B_{3,1}\right) \mathrm{d} z^{1} \wedge \mathrm{d} z^{2} \wedge \mathrm{d} z^{3} \\
& +\left(-E_{1,4}+E_{3,2}-B_{2,1}\right) \mathrm{d} z^{1} \wedge \mathrm{d} z^{2} \wedge \mathrm{d} z^{4} \\
& +\left(-E_{2,4}+E_{3,3}-B_{1,1}\right) \mathrm{d} z^{1} \wedge \mathrm{d} z^{3} \wedge \mathrm{d} z^{4} \\
& +\left(-B_{3,4}+B_{2,3}+B_{1,2}\right) \mathrm{d} z^{2} \wedge \mathrm{d} z^{3} \wedge \mathrm{d} z^{4},
\end{aligned}
$$

where the indices for the partial derivatives indicate differentiation with respect to the world coordinates $z^{k}$ (as opposed to $x^{i}$ ). Thus, in terms of time-space coordinates $\left(t, x^{i}\right)$, the four components of this equation are 


$$
\begin{array}{rrr}
\frac{\partial E_{2}}{\partial x^{1}}-\frac{\partial E_{1}}{\partial x^{2}}+\frac{\partial B_{3}}{\partial t}=0, & -\frac{\partial E_{1}}{\partial x^{3}}+\frac{\partial E_{3}}{\partial x^{1}}-\frac{\partial B_{2}}{\partial t}=0, \\
-\frac{\partial E_{2}}{\partial x^{3}}+\frac{\partial E_{3}}{\partial x^{2}}+\frac{\partial B_{1}}{\partial t}=0, & \frac{\partial B_{1}}{\partial x^{1}}+\frac{\partial B_{2}}{\partial x^{2}}+\frac{\partial B_{3}}{\partial x^{3}}=0 .
\end{array}
$$

II.3. The Lorentz Force. Let a Lorentzian frame be given in spacetime. For a particle of charge $Q$ traveling in space with velocity $v=\sum_{i} v^{i} \partial / \partial x^{i}$, we consider the 4 -velocity $\mathfrak{v}$ given locally by $\mathfrak{v}=\partial / \partial z^{1}+v^{1} \partial / \partial z^{2}+v^{2} \partial / \partial z^{3}+v^{3} \partial / \partial z^{4}$. The 4 -Lorentz force acting on the particle is given by $F=-Q \mathfrak{v}\lrcorner \mathfrak{f}$. Using Example 4.8, one has

$$
\begin{aligned}
& \frac{F}{Q}=-\left(E_{1} v^{1}+E_{2} v^{2}+E_{3} v^{3}\right) \mathrm{d} z^{1}+\left(E_{1}+v^{2} B_{3}-v^{3} B_{2}\right) \mathrm{d} z^{2} \\
&+\left(E_{2}-v^{1} B_{3}+v^{3} B_{1}\right) \mathrm{d} z^{3}+\left(E_{3}+v^{1} B_{2}-v^{2} B_{1}\right) \mathrm{d} z^{4},
\end{aligned}
$$

so that the time-like component is minus the power expanded by the electromagnetic and the three space-like components make up the traditional expression for the Lorentz force, as expected. The virtual power performed by the 4 -Lorentz force for a virtual 4 -velocity $u$ is

$$
F(u)=-(Q \mathfrak{v}\lrcorner \mathfrak{f})(u)=-Q \mathfrak{f}(\mathfrak{v}, u)
$$

and so the skew-symmetry of the Faraday 2 -form implies that

$$
F(u)=\mathfrak{f}(u, Q \mathfrak{v})=(u\lrcorner \mathfrak{f})(Q \mathfrak{v}) .
$$

We want to write an analogous expression for the Lorentz force density, a body force, induced by a charge flux $\mathfrak{J}$. Given a volume element $\theta$, one my replace the 4 -velocity above by $\iota_{\theta}(\mathfrak{J})$ and consider the $\mathrm{I}$-form $\left.-\iota_{\theta}(\mathfrak{J})\right\lrcorner \mathfrak{f}$. However, as a body force, the Lorentz force density should be a section of $L\left(T \mathscr{E}, \bigwedge^{4}\left(T^{*} \mathscr{E}\right)\right)$. We can use the inverse $\iota_{\theta}^{-1}: T \mathscr{E} \rightarrow \bigwedge^{3}\left(T^{*} \mathscr{E}\right)$ and the isomorphism $\iota_{\wedge}: \bigwedge^{1}\left(T^{*} \mathscr{E}\right) \rightarrow$ $L\left(\bigwedge^{3}\left(T^{*} \mathscr{E}\right), \bigwedge^{4}\left(T^{*} \mathscr{E}\right)\right)$ to define the Lorentz force density $\mathfrak{b}$ by $\left.\mathfrak{b}(w)=-\left(\iota_{\theta}(\mathfrak{J})\right\lrcorner \mathfrak{f}\right) \wedge$ $\left(\iota_{\theta}^{-1}(w)\right)$. Using Equation (4.IO8) we obtain the simpler expression

$$
\mathfrak{b}(w)=(w\lrcorner \mathfrak{f}) \wedge \mathfrak{J}
$$

for any 4-virtual velocity field $w$. Using Equation (4.99) in Example 4.IO, the last equation may also be written as

$$
\mathfrak{b}(w)=-\mathfrak{f} \wedge(w\lrcorner \mathfrak{J}) .
$$

It is noted that the Lorentz force density does not depend on the choice of a volume element.

The local representative of $\mathfrak{b}$ is of the form $\sum_{p} \mathfrak{b}_{1 \ldots 4 p}\left(\mathrm{~d} z^{1} \wedge \cdots \wedge \mathrm{d} z^{4}\right) \otimes \mathrm{d} z^{p}$ with $\mathfrak{b}(w)$ represented by $\sum_{p} \mathfrak{b}_{1 \ldots 4 p} w^{p} \mathrm{~d} z^{1} \wedge \cdots \wedge \mathrm{d} z^{4}$. It follows from the expression of 
the power density for the Lorentz force and from Equation (4.IO6) that

$$
\begin{aligned}
\mathfrak{b}_{1 \ldots 41} & =E_{1} \mathfrak{J}_{134}-E_{2} \mathfrak{J}_{124}+E_{3} \mathfrak{J}_{123}, \\
\mathfrak{b}_{1 \ldots 42} & =E_{1} \mathfrak{J}_{234}+B_{3} \mathfrak{J}_{124}+B_{2} \mathfrak{J}_{123}, \\
\mathfrak{b}_{1 \ldots 43} & =E_{2} \mathfrak{J}_{234}+B_{3} \mathfrak{J}_{134}-B_{1} \mathfrak{J}_{123}, \\
\mathfrak{b}_{1 \ldots 44} & =E_{3} \mathfrak{J}_{234}-B_{2} \mathfrak{J}_{134}-B_{1} \mathfrak{J}_{124} .
\end{aligned}
$$

In terms of the 3 -dimensional flux vector field $\mathbf{j}$ these may be written as

$$
\mathfrak{b}_{1 \ldots 41}=-E \cdot \mathbf{j} \vartheta_{0}, \quad \mathfrak{b}_{1 \ldots 4 i}=\vartheta_{0}\left[\rho_{123} E+(\mathbf{j} \times B)\right]_{i-1}, \quad \text { for } i>1,
$$

where $E \cdot \mathbf{j}$ denotes the inner product of vectors in $\mathbb{R}^{3}$ and $\mathbf{j} \times B$ denotes the vector product, as expected.

II.4. Metric-Invariant Maxwell Stress Tensor. We are now in a position to introduce a metric-invariant version of the Maxwell stress-energy tensor. The Maxwell stress tensor is a traction-stress field, i.e., a section $\sigma$ of $L\left(T \mathscr{E}, \bigwedge^{3} T^{*} \mathscr{E}\right)$ defined by

(cf. [Sego2, HIOo6]).

$$
\sigma(w)=(w\lrcorner \mathfrak{g}) \wedge \mathfrak{f}-(w\lrcorner \mathfrak{f}) \wedge \mathfrak{g}
$$

Using Equations (4.89) and (4.67) we may also write

$$
\begin{aligned}
\sigma(w) & =(w\lrcorner \mathfrak{g}) \wedge \mathfrak{f}-\mathfrak{g} \wedge(w\lrcorner \mathfrak{f}), \\
& =w\lrcorner(\mathfrak{g} \wedge \mathfrak{f})-2(w\lrcorner \mathfrak{f}) \wedge \mathfrak{g},
\end{aligned}
$$

etc.

Using the definition of the Maxwell stress (II.2O), the matrix of components $\left[\sigma_{1 \ldots \widehat{k} \ldots 4 r}\right]$ for the local representation $\sum_{k, r} \sigma_{1 \ldots \widehat{k} \ldots 4 r}\left(\mathrm{~d} z^{1} \wedge \cdots \wedge \widehat{\mathrm{d} z^{k}} \wedge \cdots \wedge \mathrm{d} z^{4}\right) \otimes \mathrm{d} z^{r}$, cf. (I0.26), is given by

$$
\left[\begin{array}{cccc}
B \cdot H+D \cdot E & 2(B \times D)_{1} & 2(B \times D)_{2} & 2(B \times D)_{3} \\
2(H \times E)_{1} & P_{11} & P_{12} & P_{13} \\
-2(H \times E)_{2} & P_{21} & P_{22} & P_{23} \\
2(H \times E)_{3} & P_{31} & P_{32} & P_{33}
\end{array}\right],
$$

where $P_{i j}=(-1)^{i-1}\left[(B \cdot H+D \cdot E) \delta_{i j}-2(B \otimes H+D \otimes E)_{i j}\right]$.

Let $\mathfrak{t}_{\mathfrak{R}}$ be the boundary force for a region $\mathfrak{R}$ in spacetime. Then, for each event $e \in \partial \Re, \mathfrak{t}_{\mathfrak{R}}^{\top}(e) \in \bigwedge^{3}\left(T_{e}^{*} \partial \Re, T^{*} \mathscr{E}\right)$. Let $z^{i}$ be frame coordinates in a neighborhood of $e$ and assume that $T_{e} \partial \Re$ is space-like so that its basis is of the form

$$
\left\{\frac{\partial}{\partial x^{1}}, \frac{\partial}{\partial x^{2}}, \frac{\partial}{\partial x^{3}}\right\}=\left\{\frac{\partial}{\partial z^{2}}, \frac{\partial}{\partial z^{3}}, \frac{\partial}{\partial z^{4}}\right\} .
$$

It follows that $\mathfrak{t}_{\mathfrak{R}}^{\top}(e)$ is of the form

$$
\sum_{i} \mathfrak{t}_{234 i} \mathrm{~d} z^{i} \otimes \mathrm{d} z^{2} \wedge \mathrm{d} z^{3} \wedge \mathrm{d} z^{4}
$$

which has the same form as a body force in space (rather than spacetime). Thus, the boundary 4 -force density $t$ includes the body force and there is no need to consider a 4-body force field. This is analogous to the situation with fluxes where 
the density of an extensive property is contained in the 4-flux. It follows that the principle of virtual power (I0.65) may be written in the form

$$
F_{\mathfrak{R}}(w)=\int_{\mathfrak{R}} \mathrm{d}(\sigma(w))=\int_{\partial \mathfrak{R}} \mathfrak{t}_{\mathfrak{R}}(w)=\int_{\mathfrak{R}} S(j(w)) .
$$

From the definition of the generalized divergence operator in Section I0.9,

$$
\operatorname{div} S=0 .
$$

In view of Equation (II.24), the induced virtual power density is obtained using Equation (5.62) as

$$
\mathrm{d} \sigma(w)=\mathrm{d}(w\lrcorner \mathfrak{g}) \wedge \mathfrak{f}-(w\lrcorner \mathfrak{g}) \wedge \mathrm{d} \mathfrak{f}-[\mathrm{d}(w\lrcorner \mathfrak{f}) \wedge \mathfrak{g}-(w\lrcorner \mathfrak{f}) \wedge \mathrm{d} \mathfrak{g}],
$$

and using Maxwell's equations we arrive at

$$
\mathrm{d} \sigma(w)=S(j(w))=\mathrm{d}(w\lrcorner \mathfrak{g}) \wedge \mathfrak{f}-\mathrm{d}(w\lrcorner \mathfrak{f}) \wedge \mathfrak{g}+(w\lrcorner \mathfrak{f}) \wedge \mathfrak{J} .
$$

We observe that the last term on the right is the virtual power density of the Lorentz force. It may be shown that for the case where the aether relations are used in a Lorentzian spacetime, the first two terms in Equation (II.27) cancel so that $\mathrm{d} \sigma=\mathfrak{b}$.

Using the representation (IO.3I) for $\mathrm{d} \sigma(w)$, the local components of the variational stress are given by (IO.68) which in the spacetime setting become

$$
\begin{aligned}
& R_{1 \ldots d r}=\sum_{k}(-1)^{k-1} \sigma_{1 \ldots \widehat{k} \ldots d r, k}, \\
& S_{1 \ldots d r}^{k}=(-1)^{k-1} \sigma_{1 \ldots \widehat{k} \ldots d r} .
\end{aligned}
$$

Acknowledgments. This work was partially supported by the Pearlstone Center for Aeronautical Engineering Studies at Ben-Gurion University.

\section{REFERENCES}

[AM77] L. Auslander and R.E. MacKenzie. Introduction to Differentiable Manifolds. Dover, I977.

[AMR88] R. Abraham, J.E. Marsden, and T. Ratiu. Manifolds, Tensor Analysis. and Applications. Springer, I988.

[BC70] F. Brickell and R.S. Clark. Differentiable Manifolds, an Introduction. Van Nostrand Reinhold, I970.

[Cap89] G. Capriz. Continua with Microstructure. Springer, I989.

[CMo2] G. Capriz and P.M. Mariano, editors. Advances in Multifield Theories of Continua with Substructure. Birkhauser, 2002.

[DMM99] M. Degiovanni, A. Marzocchi, and A. Musesti. Cauchy fluxes associated with tensor fields having divergence measure. Archive for Rational Mechanics and Analysis, I47:197-223, I999.

[DOo5] L. Dorfmann and R.W. Ogden. Nonlinear electroelasticity. Acta Mechanica, I74:I67-I83, 2005.

[DO09] L. Dorfmann and R.W. Ogden. Non-linear electro- and magnetoelastic interactions, in Continuum Mechanics, [Eds. J. Merodio and G. Saccomandi]. In Encyclopedia of Life Support Systems. Eolss, 2009.

[dR84] G. de Rham. Differentiable Manifolds. Springer, I984.

[Epsio] M. Epstein. The Geometrical Language of Continuum Mechanics. Cambridge University Press, 2010.

[FraO4] T. Frankel. The Geometry of Physics. Cambridge university press, 2004. 
[GWZ86] M. E. Gurtin, W. O. Williams, and W. P. Ziemer. Geometric measure theory and the axiom of continuum thermodynamics. Archive for Rational Mechanics and Analysis, 92:I-22, I986.

[HIOo6] F.W. Hehl, Y. Itin, and Y.N. Obukhov. Recent developments in premetric electrodynamics. arXiv:physics/06IO22IvI [physics.class-ph], October 2006.

[Hir76] M. Hirsch. Differential Topology. Springer, 1976.

[Kur72] K. Kuratowski. Introduction to Set Theory and Topology. Pergamon, 1972.

[LeeO2] J.M. Lee. Introduction to Smooth Manifolds. Springer-Verlag, 2002.

[LL95] L.D. Landau and E.M. Lifshitz. The Classical Theory of Fields. Course of Theoretical Physics., volume 2. Butterworth-Heinemann, I995.

[MH94] J.E. Marsden and T.J.R. Hughes. Mathematical Foundations of Elasticity. Dover, I994.

[MTW73] C.W. Misner, K.S. Thorne, and J.A. Wheeler. Gravitation. Freeman, I973.

[Mun84] J.R. Munkres. Elements of Algebraic Topology. Addison-Wesley, I984.

[Nol59] W. Noll. the foundations of classical mechanics in the light of recent advances in continuum mechanics. In Leon Henkin, Patrick Suppes, and Alfred Tarski, editors, The Axiomatic Method, with Special Reference to Geometry and Physics, pages 266-28I. North-Holland, 1959. Proceedings of an international symposium held at the University of California, Berkeley, December 26, I957-January 4, 1958.

[NV88] W. Noll and E. G. Virga. Fit regions and functions of bounded variation. Archive for Rational Mechanics and Analysis, IO2:I-2I, I988.

[Pal68] R. S. Palais. Foundations of Global Non-Linear Analysis. Benjamin, I968.

[RSO3] G. Rodnay and R. Segev. Cauchy's flux theorem in light of geometric integration theory. Journal of Elasticity, 7I:I83-203, 2003.

[Seg86] R. Segev. Forces and the existence of stresses in invariant continuum mechanics. Journal of Mathematical Physics, 27:163-I70, 1986.

[Segoo] R. Segev. The geometry of Cauchy's fluxes. Archive for Rational Mechanics and Analysis, I54:I83-I98, 2000.

[Sego2] R. Segev. Metric-independent analysis of the stress-energy tensor. Journal of Mathematical Physics, 43:3220-3231, 2002.

[Sil85] M. Silhavy. The existance of the flux vector and the divergence theorem for general cauchy flux. Archive for Rational Mechanics and Analysis, 90:I95-212, I985.

[SR99] R. Segev and G. Rodnay. Cauchy's theorem on manifolds. Journal of Elasticity, 56(2):I29-I44, I999.

[SRo2] R. Segev and G. Rodnay. Worldlines and body points associated with an extensive property. International Journal of Non-Linear Mechanics, 38:I-9, 2002.

[Tru77] C. A. Truesdell. A First Course in Rational Continuum Mechanics. Academic Press, 1977.

[TT60] C.A. Truesdell and R. Toupin. The Classical Field Theories, volume I I I/I of Handbuch der Physik. Springer, 1960 .

[War83] F.W. Warner. Foundations of Differentiable Manifolds and Lie Groups. Springer, 1983.

[Whis3] E.T. Whittaker. A History of the Theories of Aether and Electricity, vol. II., volume 2. Nelson, I953.

[Whis7] H. Whitney. Geometric Integration Theory. Princeton University Press, I957.

Current address: Reuven Segev, Department of Mechanical Engineering, Ben-Gurion University of the Negev, Beer-Sheva, Israel, rsegev@bgu.ac.il 